\title{
Assessing Potential Impacts Associated With Contamination Events in Water Distribution Systems: A Sensitivity Analysis
}

United States Environmental Protection Agency

Cincinnati, Ohio 45268 



\section{Contents}

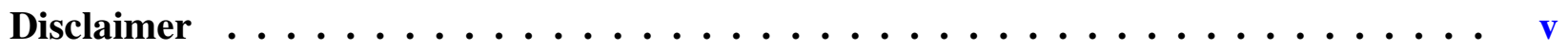

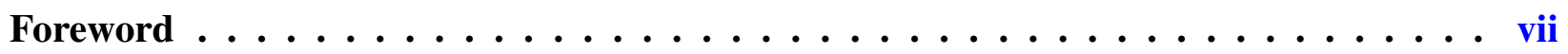

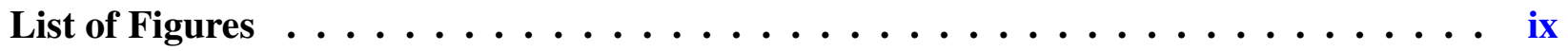

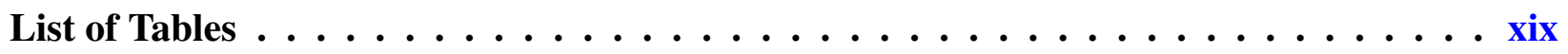

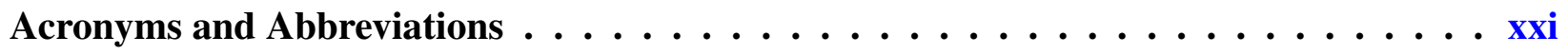

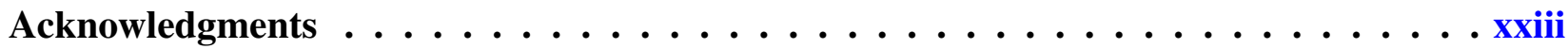

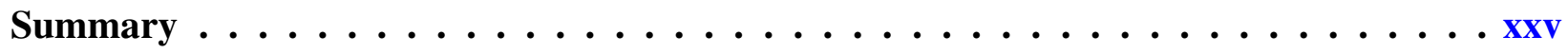

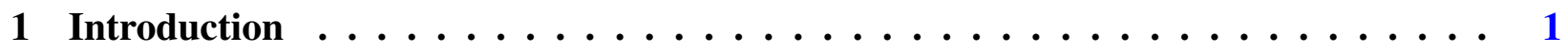

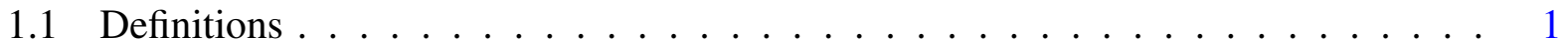

1.2 Navigating This Report . . . . . . . . . . . . . . . . . 2

2 Methodology ........................... 3

2.1 Water Distribution Systems Examined . . . . . . . . . . . . . 3

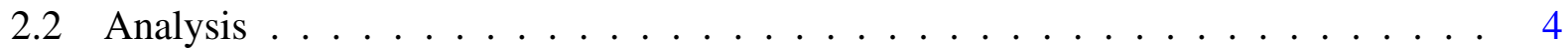

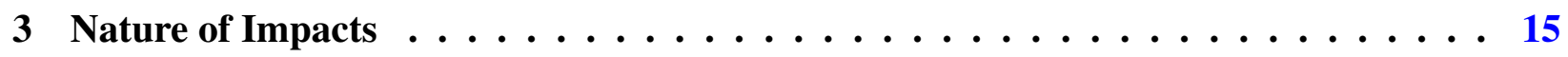

4 Sensitivity of Impacts to Injection Duration . . . . . . . . . . . . . . . . 25

5 Sensitivity of Impacts to Injection Time $\ldots \ldots \ldots \ldots \ldots$

6 Sensitivity of Impacts to Injection Mass … . . . . . . . . . . . . . . 91

7 Sensitivity of Impacts to Population Distribution … . . . . . . . . . . . 115

8 Sensitivity of Impacts to Ingestion Model $\ldots \ldots \ldots \ldots \ldots$

9 Sensitivity of Impacts to Combinations of Factors $\ldots \ldots \ldots$

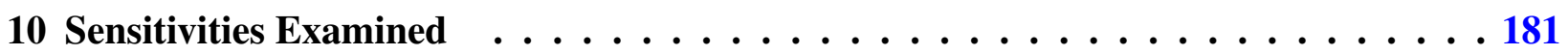




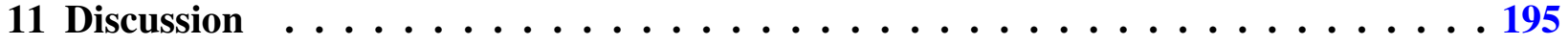

11.1 Methodological Issues . . . . . . . . . . . . . . . . . . . . 195

11.2 Computational Issues . . . . . . . . . . . . . . . . . . . . . . . . . . . . . . . . . . . . . . . . . . . . . . . . . . . . . . . .

11.3 Application of Results . . . . . . . . . . . . . . . . . . . . 198

11.4 Additional Work . . . . . . . . . . . . . . . . . . . . . . . . 198

12 Conclusions $\ldots \ldots \ldots \ldots$

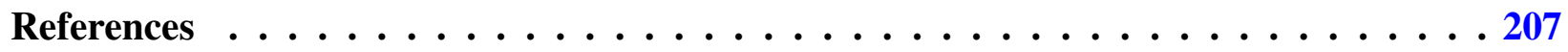

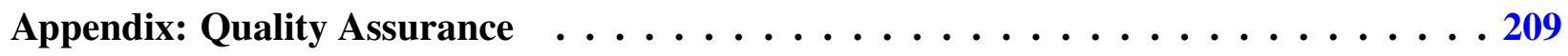




\section{Disclaimer}

The U.S. Environmental Protection Agency (EPA) through its Office of Research and Development funded, managed, and collaborated in the research described herein under an interagency agreement with the U.S. Department of Energy through Contract DE-AC02-06CH11357 with Argonne National Laboratory. It has been reviewed by the Agency but does not necessarily reflect the Agency's views. No official endorsement should be inferred. EPA does not endorse the purchase or sale of any commercial products or services.

For security reasons the identity of the water distribution systems used in this report, the associated network models, and any information that could be used to identify the systems used cannot be shared. 


\section{Foreword}

Following the events of September 11, 2001, the U.S. EPA's mission was expanded to address critical needs related to homeland security. Presidential directives identified EPA as the primary federal agency responsible for the country's water supplies and for decontamination following a chemical, biological, and/or radiological attack. To provide scientific and technical support in meeting this expanded mission, EPA's National Homeland Security Research Center (NHSRC) was established. NHSRC is focused on conducting research and delivering products that improve the capability of the Agency to carry out its homeland security responsibilities.

As a part of this mission, NHSRC conducts research and provides technical assistance to support America's drinking water utilities so that they can improve their security preparedness, response, and recovery. Over the last several years, NHSRC has been developing new methods to help design, implement, and evaluate drinking water contamination warning systems. These new systems integrate a variety of monitoring technologies to rapidly detect contamination. Water distribution system networks are composed of hundreds to thousands of miles of pipe and the contamination warning system must economically safeguard the largest number of people reasonably possible. Designers of contamination warning systems need to understand how key factors associated with contamination events affect public health outcomes. For example, how does the location of the contaminant release, the toxicity of the contaminant, or the length of time over which the contaminant is introduced into the water system influence the number of people affected? This publication presents a sensitivity analysis of the critical parameters associated with contamination events. The work includes extensive data and information on the vulnerability of drinking water distribution systems. The results were obtained by simulating contamination events for 12 real, diverse water systems.

This report results from collaborative research between the NHSRC and Argonne National Laboratory. It is published and made available by EPA's Office of Research and Development in order to enable the water community to improve the security of our nation's drinking water.

Jonathan Herrmann, Director

National Homeland Security Research Center 


\section{List of Figures}

2.1 Distribution of nodal populations for the networks below 250 persons per node. . . 5

2.2 Network 4. . . . . . . . . . . . . . . . . . . 6

2.3 Network 6. . . . . . . . . . . . . . . . . . . 7

2.4 Overlap of high-percentile injection nodes. . . . . . . . . . . . . 10

2.5 Comparison of a violin plot and a box plot. . . . . . . . . . . . . . . 12

3.1 Impacts for Networks 1 to 4 as function of dose level. . . . . . . . . . . . 16

3.2 Impacts for Networks 5 to 8 as function of dose level. . . . . . . . . . . . 17

3.3 Impacts Networks 9 to 12 as function of dose level. . . . . . . . . . . . . 18

3.4 Network 4 showing locations of injection nodes associated with $90^{\text {th }}$ and $95^{\text {th }}$ percentile or higher impacts at a dose level of $0.0001 \mathrm{mg}$. . . . . . . . . . . 20

3.5 Network 4 showing locations of injection nodes associated with $85^{\text {th }}$ and $95^{\text {th }}$

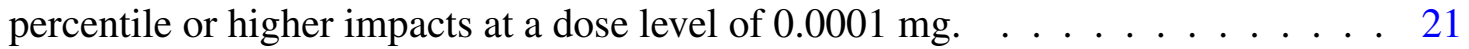

3.6 Network 4 showing locations of injection nodes associated with $80^{\text {th }}$ and $95^{\text {th }}$ percentile or higher impacts at a dose level of $0.0001 \mathrm{mg}$. . . . . . . . . . . . . 22

3.7 Network 6 showing receptor nodes affected following injections at the $90^{\text {th }}$ percentile nodes for dose levels of 0.0001 and $1.0 \mathrm{mg}$. . . . . . . . . . . . . . 23

4.1 Impacts associated with 1-h and 24-h injections for Network 12 as a function of dose level, for the $90^{\text {th }}, 95^{\text {th }}, 99^{\text {th }}$, and $100^{\text {th }}$ percentile injection nodes. . . . . . . . 28

4.2 Impacts associated with 1-h and 24-h injections for Network 12 as a function of dose level, for the $50^{\text {th }}, 60^{\text {th }}, 70^{\text {th }}$, and $80^{\text {th }}$ percentile injection nodes. . . . . . . . 29

4.3 Impact for a 24-h injection relative to the impact for a 1-h injection, as a function of dose level, for the $90^{\text {th }}, 95^{\text {th }}, 99^{\text {th }}$, and $100^{\text {th }}$ percentile injection nodes. . . . . . 30

4.4 Impact for a 24-h injection relative to the impact for a 1-h injection, as a function of dose level, for the $50^{\text {th }}, 60^{\text {th }}, 70^{\text {th }}$, and $80^{\text {th }}$ percentile injection nodes. . . . . . 31

4.5 Distributions of impacts for Network 12 across all injection nodes for 1-h and 24-h injections for dose levels of $0.0001,0.001,0.01$, and $0.1 \mathrm{mg} . \quad \ldots . . .32$

4.6 Distributions of impacts for Network 12 across all injection nodes for 1-h and 24-h injections for dose levels of 1,10 , and $100 \mathrm{mg}$. . . . . . . . . . . . . 33

4.7 Impacts for Network 12 for 1 -h and 24-h injections for dose levels of 0.0001 , $0.001,0.01$, and $0.1 \mathrm{mg} . \ldots \ldots \ldots$. . . . . . . . . . . . . . . . . . . . . . . .

4.8 Impacts for Network 12 for 1-h and 24-h injections for dose levels of 1, 10, and $100 \mathrm{mg}$. . . . . . . . . . . . . . . . . . 35

4.9 Impacts for Network 12 that are at or above the $80^{\text {th }}$ percentile level for 1 -h and/or 24-h injections for dose levels of $0.0001,0.001,0.01$, and $0.1 \mathrm{mg}$. . . . . . . . 36 
4.10 Impacts for Network 12 that are at or above the $80^{\text {th }}$ percentile level for 1-h and/or 24-h injections for dose levels of 1,10 , and $100 \mathrm{mg} . \ldots \ldots 37$

4.11 Overlap of high percentile injection nodes for Network 12 for 1-h and 24-h injections for different dose levels. . . . . . . . . . . . . . . . . 38

4.12 Overlap of high percentile injection nodes for the networks for 1-h and 24-h injections for dose levels of $0.0001,0.001,0.01$, and $0.1 \mathrm{mg}$. . . . . . . . . . 39

4.13 Overlap of high percentile injection nodes for the networks for 1-h and 24-h injections for dose levels of 1,10 , and $100 \mathrm{mg}$. . . . . . . . . . . . . 40

4.14 Network 4 showing locations of injection nodes associated with $95^{\text {th }}$ percentile or higher impacts at a dose level of $0.0001 \mathrm{mg}$ for injection durations of $1 \mathrm{~h}$ and $24 \mathrm{~h} . \quad 41$

4.15 Network 4 showing locations of injection nodes associated with $95^{\text {th }}$ percentile or higher impacts at a dose level of $1.0 \mathrm{mg}$ for injection durations of $1 \mathrm{~h}$ and $24 \mathrm{~h} . ~ . ~ .42$

4.16 Network 6 showing locations of injection nodes associated with $95^{\text {th }}$ percentile or higher impacts at a dose level of $0.0001 \mathrm{mg}$ for injection durations of $1 \mathrm{~h}$ and $24 \mathrm{~h} . \quad 43$

4.17 Network 6 showing locations of injection nodes associated with $95^{\text {th }}$ percentile or higher impacts at a dose level of $1.0 \mathrm{mg}$ for injection durations of $1 \mathrm{~h}$ and $24 \mathrm{~h}$. . . 44

5.1 Impacts associated with different injection times for Network 12 as a function of dose level, for the $90^{\text {th }}, 95^{\text {th }}, 99^{\text {th }}$, and $100^{\text {th }}$ percentile injection nodes. . . . . . . 47

5.2 Impacts associated with different injection times for Network 12 as a function of dose level, for the $50^{\text {th }}, 60^{\text {th }}, 70^{\text {th }}$, and $80^{\text {th }}$ percentile injection nodes. . . . . . . 48

5.3 Maximum ratio of impacts for the networks for four different injection times as a function of dose level, for the $90^{\text {th }}, 95^{\text {th }}, 99^{\text {th }}$, and $100^{\text {th }}$ percentile injection nodes.

5.4 Maximum ratio of impacts for the networks for four different injection times as a function of dose level, for the $50^{\text {th }}, 60^{\text {th }}, 70^{\text {th }}$, and $80^{\text {th }}$ percentile injection nodes. . 50

5.5 Relative impacts for the 12 networks for different injection times, for a dose level of $0.0001 \mathrm{mg}$ and for injection nodes associated with different percentile impacts.

5.6 Relative impacts for the 12 networks for different injection times, for a dose level of $0.001 \mathrm{mg}$ and for injection nodes associated with different percentile impacts. . 52

5.7 Relative impacts for the 12 networks for different injection times, for a dose level of $0.01 \mathrm{mg}$ and for injection nodes associated with different percentile impacts. . . 53

5.8 Relative impacts for the 12 networks for different injection times, for a dose level of $0.1 \mathrm{mg}$ and for injection nodes associated with different percentile impacts. . . 54

5.9 Relative impacts for the 12 networks for different injection times, for a dose level of $1 \mathrm{mg}$ and for injection nodes associated with different percentile impacts. . . . 55

5.10 Distributions of impacts for Network 12 across all injection nodes for different injection times, for dose levels of $0.0001,0.001,0.01$, and $0.1 \mathrm{mg}$. . . . . . . . 56

5.11 Distributions of impacts for Network 12 across all injection nodes for different injection times, for dose levels of 1,10 , and $100 \mathrm{mg}$. . . . . . . . . . . . 57

5.12 Impacts for Network 12 for injection times of 0:00 and 6:00, for dose levels of $0.0001,0.001,0.01$, and $0.1 \mathrm{mg} \ldots \ldots \ldots \ldots \ldots$

5.13 Impacts for Network 12 for injection times of 0:00 and 6:00, for dose levels of 1, 10 , and $100 \mathrm{mg} . \ldots \ldots \ldots \ldots \ldots \ldots \ldots$

5.14 Impacts for Network 12 for injection times of 0:00 and 12:00, for dose levels of $0.0001,0.001,0.01$, and $0.1 \mathrm{mg} \ldots \ldots \ldots$. . . . . . . . . 60 
5.15 Impacts for Network 12 for injection times of 0:00 and 12:00, for dose levels of

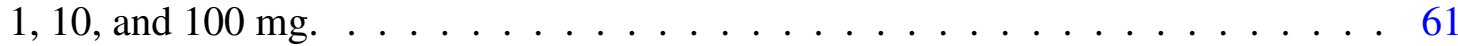

5.16 Impacts for Network 12 for injection times of 0:00 and 18:00, for dose levels of $0.0001,0.001,0.01$, and $0.1 \mathrm{mg} \ldots \ldots \ldots$. . . . . . . . . . . 62

5.17 Impacts for Network 12 for injection times of 0:00 and 18:00, for dose levels of 1,10 , and $100 \mathrm{mg} . \ldots \ldots \ldots \ldots \ldots$

5.18 Impacts for Network 12 that are at or above the $80^{\text {th }}$ percentile level for an injection time of 6:00 and/or an injection time of 0:00, for dose levels of 0.0001, 0.001, 0.01 , and $0.1 \mathrm{mg} \ldots \ldots \ldots \ldots \ldots \ldots$

5.19 Impacts for Network 12 that are at or above the $80^{\text {th }}$ percentile level for an injection time of 6:00 and/or an injection time of 0:00, for dose levels of 1, 10, and 100 mg. . . . . . . . . . . . . . . . . . . .

5.20 Impacts for Network 12 that are at or above the $80^{\text {th }}$ percentile level for an injection time of 12:00 and/or an injection time of 0:00, for dose levels of 0.0001, $0.001,0.01$, and $0.1 \mathrm{mg} . \ldots \ldots \ldots \ldots$

5.21 Impacts for Network 12 that are at or above the $80^{\text {th }}$ percentile level for an injection time of 12:00 and/or an injection time of 0:00, for dose levels of 1, 10, and $100 \mathrm{mg} \ldots \ldots \ldots \ldots \ldots \ldots$

5.22 Impacts for Network 12 that are at or above the $80^{\text {th }}$ percentile level for an injection time of 18:00 and/or an injection time of 0:00, for dose levels of 0.0001, $0.001,0.01$, and $0.1 \mathrm{mg} . \ldots \ldots \ldots \ldots$

5.23 Impacts for Network 12 that are at or above the $80^{\text {th }}$ percentile level for an injection time of 18:00 and/or an injection time of 0:00, for dose levels of 1, 10, and $100 \mathrm{mg} \ldots \ldots \ldots \ldots \ldots \ldots \ldots$

5.24 Overlap of high percentile injection nodes for Network 12 for an injection time of 6:00 with those for an injection time of 0:00 for different dose levels. . . . . . . . 70

5.25 Overlap of high percentile injection nodes for Network 12 for an injection time of 12:00 with those for an injection time of 0:00 for different dose levels. . . . . . . . 71

5.26 Overlap of high percentile injection nodes for Network 12 for an injection time of 18:00 with those for an injection time of 0:00 for different dose levels. . . . . . . . 72

5.27 Overlap of high percentile injection nodes for the networks for an injection time of 6:00 with those for an injection time of 0:00, for dose levels of 0.0001, 0.001,

5.28 Overlap of high percentile injection nodes for the networks for an injection time of 6:00 with those for an injection time of 0:00, for dose levels of 1, 10, and $100 \mathrm{mg} .74$

5.29 Overlap of high percentile injection nodes for the networks for an injection time of 12:00 with those for an injection time of 0:00, for dose levels of 0.0001, 0.001, 0.01 , and $0.1 \mathrm{mg} . \ldots \ldots \ldots \ldots \ldots$

5.30 Overlap of high percentile injection nodes for the networks for an injection time

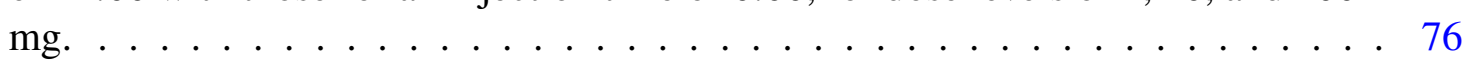

5.31 Overlap of high percentile injection nodes for the networks for an injection time of 18:00 with those for an injection time of 0:00, for dose levels of 0.0001, 0.001, 0.01 , and $0.1 \mathrm{mg} \ldots \ldots \ldots \ldots \ldots \ldots$ 
5.32 Overlap of high percentile injection nodes for the networks for an injection time of 18:00 with those for an injection time of 0:00, for dose levels of 1,10 , and 100 mg. . . . . . . . . . . . . . . . . . . . 78

5.33 Network 4 showing locations of injection nodes associated with $95^{\text {th }}$ percentile or higher impacts at a dose level of $0.0001 \mathrm{mg}$ for injection times of 0:00 and 6:00. . 79

5.34 Network 4 showing locations of injection nodes associated with $95^{\text {th }}$ percentile or higher impacts at a dose level of $0.0001 \mathrm{mg}$ for injection times of 0:00 and 12:00. . 80

5.35 Network 4 showing locations of injection nodes associated with $95^{\text {th }}$ percentile or higher impacts at a dose level of 0.0001 mg for injection times of 0:00 and 18:00. . 81

5.36 Network 4 showing locations of injection nodes associated with $95^{\text {th }}$ percentile or higher impacts at a dose level of $1.0 \mathrm{mg}$ for injection times of 0:00 and 6:00. . . . 82

5.37 Network 4 showing locations of injection nodes associated with $95^{\text {th }}$ percentile or higher impacts at a dose level of $1.0 \mathrm{mg}$ for injection times of 0:00 and 12:00. . . . 83

5.38 Network 4 showing locations of injection nodes associated with $95^{\text {th }}$ percentile or higher impacts at a dose level of $1.0 \mathrm{mg}$ for injection times of 0:00 and 18:00. . . 84

5.39 Network 6 showing locations of injection nodes associated with $95^{\text {th }}$ percentile or higher impacts at a dose level of $0.0001 \mathrm{mg}$ for injection times of 0:00 and 6:00. . 85

5.40 Network 6 showing locations of injection nodes associated with $95^{\text {th }}$ percentile or higher impacts at a dose level of $0.0001 \mathrm{mg}$ for injection times of 0:00 and 12:00. . 86

5.41 Network 6 showing locations of injection nodes associated with $95^{\text {th }}$ percentile or higher impacts at a dose level of $0.0001 \mathrm{mg}$ for injection times of 0:00 and 18:00. . 87

5.42 Network 6 showing locations of injection nodes associated with $95^{\text {th }}$ percentile or higher impacts at a dose level of $1.0 \mathrm{mg}$ for injection times of 0:00 and 6:00. . . 88

5.43 Network 6 showing locations of injection nodes associated with $95^{\text {th }}$ percentile or higher impacts at a dose level of $1.0 \mathrm{mg}$ for injection times of 0:00 and 12:00. . . 89

5.44 Network 6 showing locations of injection nodes associated with $95^{\text {th }}$ percentile or higher impacts at a dose level of $1.0 \mathrm{mg}$ for injection times of 0:00 and 18:00 . . . 90

6.1 Impacts associated with injection masses of $1 \mathrm{X}$ and 10X for Network 12 as a function of dose level, for the $90^{\text {th }}, 95^{\text {th }}, 99^{\text {th }}$, and $100^{\text {th }}$ percentile injection nodes.

6.2 Impacts associated with injection masses of $1 \mathrm{X}$ and 10X for Network 12 as a function of dose level, for the $50^{\text {th }}, 60^{\text {th }}, 70^{\text {th }}$, and $80^{\text {th }}$ percentile injection nodes. . 94

6.3 Impact for a 10X injection mass relative to the impact for a $1 \mathrm{X}$ injection mass, as a function of dose level, for the $90^{\text {th }}, 95^{\text {th }}, 99^{\text {th }}$, and $100^{\text {th }}$ percentile injection nodes. 95

6.4 Impact for a 10X injection mass relative to the impact for a $1 \mathrm{X}$ injection mass, as a function of dose level, for the $50^{\text {th }}, 60^{\text {th }}, 70^{\text {th }}$, and $80^{\text {th }}$ percentile injection nodes. 96

6.5 Variation in impacts as a function of injection mass for a dose level of $0.0001 \mathrm{mg} . \quad 97$

6.6 Variation in impacts as a function of injection mass for a dose level of $0.001 \mathrm{mg}$. . 98

6.7 Variation in impacts as a function of injection mass for a dose level of $0.01 \mathrm{mg}$. . . 99

6.8 Variation in impacts as a function of injection mass for a dose level of $0.1 \mathrm{mg}$. . . 100

6.9 Variation in impacts as a function of injection mass for a dose level of $1 \mathrm{mg} . \quad$. . . 101

6.10 Distributions of impacts for Network 12 across all injection nodes for injection masses of $1 \mathrm{X}$ and 10X, for dose levels of $0.0001,0.001,0.01$, and $0.1 \mathrm{mg} . \quad \ldots 102$

6.11 Distributions of impacts for Network 12 across all injection nodes for injection masses of $1 \mathrm{X}$ and 10X, for dose levels of 1,10 , and $100 \mathrm{mg}$. . . . . . . . 103 
6.12 Impacts for Network 12 for injection masses of $1 \mathrm{X}$ and $10 \mathrm{X}$, for dose levels of $0.0001,0.001,0.01$, and $0.1 \mathrm{mg}$. . . . . . . . . . . . . . . . 104

6.13 Impacts for Network 12 for injection masses of $1 \mathrm{X}$ and 10X, for dose levels of 1 ,

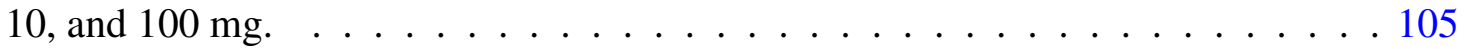

6.14 Impacts for Network 12 that are at or above the $80^{\text {th }}$ percentile level for $1 \mathrm{X}$ and/or $10 \mathrm{X}$ injection masses, for dose levels of $0.0001,0.001,0.01$, and $0.1 \mathrm{mg}$. . . . . 106

6.15 Impacts for Network 12 that are at or above the $80^{\text {th }}$ percentile level for $1 \mathrm{X}$ and/or 10X injection masses, for dose levels of 1,10 , and $100 \mathrm{mg}$. . . . . . . . . . . . 107

6.16 Overlap of high percentile injection nodes for Network 12 for injection masses of $1 \mathrm{X}$ and 10X for different dose levels. . . . . . . . . . . . . . . . . 108

6.17 Overlap of high percentile injection nodes for the networks for injection masses of $1 \mathrm{X}$ and 10X, for dose levels of $0.0001,0.001,0.01$, and $0.1 \mathrm{mg}$. . . . . . . . . 109

6.18 Overlap of high percentile injection nodes for the networks for injection masses of $1 \mathrm{X}$ and 10X, for dose levels of 1,10 , and $100 \mathrm{mg}$. . . . . . . . . . . . . 110

6.19 Network 4 showing locations of injection nodes associated with $95^{\text {th }}$ percentile or higher impacts at a dose level of $0.0001 \mathrm{mg}$ for injection masses of $1 \mathrm{X}$ and 10X. . 111

6.20 Network 4 showing locations of injection nodes associated with $95^{\text {th }}$ percentile or higher impacts at a dose level of $1.0 \mathrm{mg}$ for injection masses of $1 \mathrm{X}$ and $10 \mathrm{X}$. . . . 112

6.21 Network 6 showing locations of injection nodes associated with $95^{\text {th }}$ percentile or higher impacts at a dose level of $0.0001 \mathrm{mg}$ for injection masses of $1 \mathrm{X}$ and 10X. . 113

6.22 Network 6 showing locations of injection nodes associated with $95^{\text {th }}$ percentile or higher impacts at a dose level of $1.0 \mathrm{mg}$ for injection masses of $1 \mathrm{X}$ and 10X. . . . 114

7.1 Impacts associated with different population models for Network 12 as a function of dose level, for the $90^{\text {th }}, 95^{\text {th }}, 99^{\text {th }}$, and $100^{\text {th }}$ percentile injection nodes. . . . . . 117

7.2 Impacts associated with different population models for Network 12 as a function of dose level, for the $50^{\text {th }}, 60^{\text {th }}, 70^{\text {th }}$, and $80^{\text {th }}$ percentile injection nodes. . . . . . . 118

7.3 Relative magnitudes of the impacts for the networks obtained with different population models as a function of dose level, for the $90^{\text {th }}, 95^{\text {th }}, 99^{\text {th }}$, and $100^{\text {th }}$ percentile injection nodes. . . . . . . . . . . . . . . . . . . . . . 119

7.4 Relative magnitudes of the impacts for the networks obtained with different population models as a function of dose level, for the $50^{\text {th }}, 60^{\text {th }}, 70^{\text {th }}$, and $80^{\text {th }}$ percentile injection nodes. . . . . . . . . . . . . . . . . . . 120

7.5 Distributions of impacts for Network 12 across all injection nodes for different population models, for dose levels of $0.0001,0.001,0.01$, and $0.1 \mathrm{mg}$. . . . . . . . 121

7.6 Distributions of impacts for Network 12 across all injection nodes for different population models, for dose levels of 1,10 , and $100 \mathrm{mg}$. . . . . . . . . . . . 122

7.7 Impacts for Network 12 obtained with different population models, for dose levels of $0.0001,0.001,0.01$, and $0.1 \mathrm{mg}$. . . . . . . . . . . . . . 123

7.8 Impacts for Network 12 obtained with different population models, for dose levels of 1,10 , and $100 \mathrm{mg}$. . . . . . . . . . . . . . . . . . . . 124

7.9 Impacts for Network 12 that are at or above the $80^{\text {th }}$ percentile level obtained with different population models, for dose levels of $0.0001,0.001,0.01$, and $0.1 \mathrm{mg}$. . . 125

7.10 Impacts for Network 12 that are at or above the $80^{\text {th }}$ percentile level obtained with different population models, for dose levels of 1,10 , and $100 \mathrm{mg}$. . . . . . . . 126 
7.11 Overlap of high percentile injection nodes for Network 12 for the two population models for different dose levels. . . . . . . . . . . . . . . . . . . 127

7.12 Overlap of high percentile injection nodes for the networks for the two population models, for dose levels of $0.0001,0.001,0.01$, and $0.1 \mathrm{mg}$. . . . . . . . . . . 128

7.13 Overlap of high percentile injection nodes for the networks for the two population models, for dose levels of 1,10 , and $100 \mathrm{mg}$. . . . . . . . . . . . . . 129

7.14 Network 4 showing locations of injection nodes associated with $95^{\text {th }}$ percentile or higher impacts at a dose level of $0.0001 \mathrm{mg}$ for the two population models. . . . . 130

7.15 Network 4 showing locations of injection nodes associated with $95^{\text {th }}$ percentile or higher impacts at a dose level of $1.0 \mathrm{mg}$ for the two population models. . . . . . . 131

7.16 Network 6 showing locations of injection nodes associated with $95^{\text {th }}$ percentile or higher impacts at a dose level of $0.0001 \mathrm{mg}$ for the two population models. . . . . 132

7.17 Network 6 showing locations of injection nodes associated with $95^{\text {th }}$ percentile or higher impacts at a dose level of $1.0 \mathrm{mg}$ for the two population models. . . . . . . 133

8.1 Impacts associated with different ingestion models for Network 12 as a function of dose level, for the $90^{\text {th }}, 95^{\text {th }}, 99^{\text {th }}$, and $100^{\text {th }}$ percentile injection nodes. . . . . 137

8.2 Impacts associated with different ingestion models for Network 12 as a function of dose level, for $50^{\text {th }}, 60^{\text {th }}, 70^{\text {th }}$, and $80^{\text {th }}$ percentile injection nodes. . . . . . . 138

8.3 Relative magnitudes of the impacts for the networks obtained with different ingestion models as a function of dose level, for the $90^{\text {th }}, 95^{\text {th }}, 99^{\text {th }}$, and $100^{\text {th }}$ percentile injection nodes. . . . . . . . . . . . . . . . . . . . . . 139

8.4 Relative magnitudes of the impacts for the networks obtained with different ingestion models as a function of dose level, for $50^{\text {th }}, 60^{\text {th }}, 70^{\text {th }}$, and $80^{\text {th }}$ percentile injection nodes. . . . . . . . . . . . . . . . . . . 140

8.5 Distributions of impacts for Network 12 across all injection nodes for different ingestion models, for dose levels of $0.0001,0.001,0.01$, and $0.1 \mathrm{mg}$. . . . . . . 141

8.6 Distributions of impacts for Network 12 across all injection nodes for different ingestion models, for dose levels of 1,10 , and $100 \mathrm{mg}$. . . . . . . . . . . . . 142

8.7 Impacts for Network 12 obtained with different ingestion models, for dose levels of $0.0001,0.001,0.01$, and $0.1 \mathrm{mg}$. . . . . . . . . . . . . . . 143

8.8 Impacts for Network 12 obtained with different ingestion models, for dose levels of 1,10 , and $100 \mathrm{mg}$. . . . . . . . . . . . . . . . . . . . . . . . 144

8.9 Impacts for Network 12 that are at or above the $80^{\text {th }}$ percentile level obtained with different ingestion models, for dose levels of $0.0001,0.001,0.01$, and $0.1 \mathrm{mg}$. . . 145

8.10 Impacts for Network 12 that are at or above the $80^{\text {th }}$ percentile level obtained with different ingestion models, for dose levels of 1,10 , and $100 \mathrm{mg}$. . . . . . . . . 146

8.11 Overlap of high percentile injection nodes for Network 12 for different ingestion models for different dose levels. . . . . . . . . . . . . . . . . . . . 147

8.12 Overlap of high percentile injection nodes for the networks for the two ingestion models, for dose levels of $0.0001,0.001,0.01$, and $0.1 \mathrm{mg}$. . . . . . . . . . 148

8.13 Overlap of high percentile injection nodes for the networks for the two ingestion models, for dose levels of 1,10 , and $100 \mathrm{mg}$. . . . . . . . . . . . . 149

8.14 Network 4 showing locations of injection nodes associated with $95^{\text {th }}$ percentile or higher impacts at a dose level of $0.0001 \mathrm{mg}$ for the two ingestion models. . . . . . 150 
8.15 Network 4 showing locations of injection nodes associated with $95^{\text {th }}$ percentile or higher impacts at a dose level of $1.0 \mathrm{mg}$ for the two ingestion models. . . . . . . . 151

8.16 Network 6 showing locations of injection nodes associated with $95^{\text {th }}$ percentile or higher impacts at a dose level of $0.0001 \mathrm{mg}$ for the two ingestion models. . . . . . 152

8.17 Network 6 showing locations of injection nodes associated with $95^{\text {th }}$ percentile or higher impacts at a dose level of $1.0 \mathrm{mg}$ for the two ingestion models. . . . . . . 153

9.1 Histograms of impacts for the 32 combinations of factors for Network 2 for a dose level of $0.0001 \mathrm{mg}$ and different impact levels. . . . . . . . . . . . . 157

9.2 Histograms of impacts for the 32 combinations of factors for Network 2 for a dose level of $0.01 \mathrm{mg}$ and different impact levels. . . . . . . . . . . . . . . 158

9.3 Histograms of impacts for the 32 combinations of factors for Network 2 for a dose level of $1 \mathrm{mg}$ and different impact levels. . . . . . . . . . . . . . . . 159

9.4 Histograms of impacts for the 32 combinations of factors for Network 2 for a dose level of $100 \mathrm{mg}$ and different impact levels. . . . . . . . . . . . . . . . 160

9.5 Histograms of impacts for the 32 combinations of factors for Network 2 for a dose level of $0.0001 \mathrm{mg}$ and different impact levels with cases color coded to show associated injection time. . . . . . . . . . . . . . . . . . . . 161

9.6 Histograms of impacts for the 32 combinations of factors for Network 2 for a dose level of $0.01 \mathrm{mg}$ and different impact levels with cases color coded to show associated injection time. . . . . . . . . . . . . . . . . . . 162

9.7 Histograms of impacts for the 32 combinations of factors for Network 2 for a dose level of $1 \mathrm{mg}$ and different impact levels with cases color coded to show associated injection time.

9.8 Histograms of impacts for the 32 combinations of factors for Network 2 for a dose level of $100 \mathrm{mg}$ and different impact levels with cases color coded to show associated injection time. . . . . . . . . . . . . . . . . . . 164

9.9 Histograms of impacts for the 32 combinations of factors for Network 2 for a dose level of $0.0001 \mathrm{mg}$ and different impact levels with cases color coded to show associated injection duration.

9.10 Histograms of impacts for the 32 combinations of factors for Network 2 for a dose level of $0.01 \mathrm{mg}$ and different impact levels with cases color coded to show associated injection duration.

9.11 Histograms of impacts for the 32 combinations of factors for Network 2 for a dose level of $1 \mathrm{mg}$ and different impact levels with cases color coded to show associated injection duration.

9.12 Histograms of impacts for the 32 combinations of factors for Network 2 for a dose level of $100 \mathrm{mg}$ and different impact levels with cases color coded to show associated injection duration.

9.13 Histograms of impacts for the 32 combinations of factors for Network 2 for a dose level of $0.0001 \mathrm{mg}$ and different impact levels with cases color coded to show associated injection mass.

9.14 Histograms of impacts for the 32 combinations of factors for Network 2 for a dose level of $0.01 \mathrm{mg}$ and different impact levels with cases color coded to show associated injection mass. 
9.15 Histograms of impacts for the 32 combinations of factors for Network 2 for a dose level of $1 \mathrm{mg}$ and different impact levels with cases color coded to show associated injection mass. . . . . . . . . . . . . . . . . . . . . 17

9.16 Histograms of impacts for the 32 combinations of factors for Network 2 for a dose level of $100 \mathrm{mg}$ and different impact levels with cases color coded to show associated injection mass. . . . . . . . . . . . . . . . . . . . 172

9.17 Histograms of impacts for the 32 combinations of factors for Network 2 for a dose level of $0.0001 \mathrm{mg}$ and different impact levels with cases color coded to show which population model was used.

9.18 Histograms of impacts for the 32 combinations of factors for Network 2 for a dose level of $0.01 \mathrm{mg}$ and different impact levels with cases color coded to show which population model was used.

9.19 Histograms of impacts for the 32 combinations of factors for Network 2 for a dose level of $1 \mathrm{mg}$ and different impact levels with cases color coded to show which population model was used.

9.20 Histograms of impacts for the 32 combinations of factors for Network 2 for a dose level of $100 \mathrm{mg}$ and different impact levels with cases color coded to show which population model was used.

9.21 Overlap of injection nodes for the 32 combinations of factors for Network 2 for different dose levels for $75^{\text {th }}$ percentile and higher impacts. . . . . . . . . . . . . 177

9.22 Overlap of injection nodes for the 32 combinations of factors for Network 2 for different dose levels for $95^{\text {th }}$ percentile and higher impacts. . . . . . . . . . . 178

9.23 Overlap of injection nodes for the 32 combinations of factors for Network 2 for different dose levels for $99^{\text {th }}$ percentile and higher impacts. . . . . . . . . . . . 179

10.1 Summary of the ratio of $95^{\text {th }}$ percentile impacts associated with 1- and 24-h injections for the networks for two dose levels . . . . . . . . . . . . . . 182

10.2 Summary of the maximum ratio of $95^{\text {th }}$ percentile impacts associated with different injection times $(0: 00,6: 00,12: 00$, and 18:00) for the networks for two dose levels . . . . . . . . . . . . . . . . . . . . 183

10.3 Summary of the ratio of $95^{\text {th }}$ percentile impacts associated with different injection masses (1X and 10X) for the networks for two dose levels . . . . . . . . . . . . 184

10.4 Summary of the ratio of $95^{\text {th }}$ percentile impacts associated with the two population models for the networks for two dose levels . . . . . . . . . . . . . . . 185

10.5 Summary of the ratio of $95^{\text {th }}$ percentile impacts associated with the two ingestion models for the networks for two dose levels . . . . . . . . . . . . . . 186

10.6 Summary of the overlap of $95^{\text {th }}$ percentile and higher injection nodes associated with 1-h and 24-h injections for the networks . . . . . . . . . . . . 187

10.7 Summary of the overlap of $95^{\text {th }}$ percentile and higher injection nodes associated with injection times of $0: 00$ and $6: 00$ for the networks $\ldots \ldots \ldots 188$

10.8 Summary of the overlap of $95^{\text {th }}$ percentile and higher injection nodes associated with injection times of 0:00 and 12:00 for the networks . . . . . . . . . . 189

10.9 Summary of the overlap of $95^{\text {th }}$ percentile and higher injection nodes associated with injection times of 0:00 and 18:00 for the networks . . . . . . . . . . 190 
10.10 Summary of the overlap of $95^{\text {th }}$ percentile and higher injection nodes associated with 1X and 10X injection masses for the networks . . . . . . . . . . . . . 191

10.11 Summary of the overlap of $95^{\text {th }}$ percentile and higher injection nodes for the networks for the two population models . . . . . . . . . . . . . . . 192

10.12 Summary of the overlap of $95^{\text {th }}$ percentile and higher injection nodes for the networks for the two ingestion models . . . . . . . . . . . . . 193

11.1 Network 4 showing locations of injection nodes associated with $95^{\text {th }}$ percentile or higher impacts at dose levels of $0.0001 \mathrm{mg}$ and $0.001 \mathrm{mg}$. . . . . . . . . . 200

11.2 Network 4 showing locations of injection nodes associated with $95^{\text {th }}$ percentile or higher impacts at dose levels of $0.0001 \mathrm{mg}$ and $0.01 \mathrm{mg}$. . . . . . . . . . . 201

11.3 Network 4 showing locations of injection nodes associated with $95^{\text {th }}$ percentile or higher impacts at dose levels of $0.0001 \mathrm{mg}$ and $0.1 \mathrm{mg}$. . . . . . . . . . . 202

11.4 Network 4 showing locations of injection nodes associated with $95^{\text {th }}$ percentile or higher impacts at dose levels of $0.0001 \mathrm{mg}$ and $1 \mathrm{mg}$. . . . . . . . . . . 203 
Xviii

LIST OF FIGURES 


\section{List of Tables}

2.1 System Descriptions . . . . . . . . . . . . . . . . . . . . . . . . . . 4

2.2 Ingestion Models . . . . . . . . . . . . . . . . . . . . . . 9

2.3 Factors Considered in the Sensitivity Analysis . . . . . . . . . . . . . . 9 


\section{Acronyms and Abbreviations}

Acronym
CWS
EPA
EPANET
NZD
QA
QC
TEVA-SPOT

\section{Definition}

Contamination Warning System

Environmental Protection Agency

name of software

non-zero demand (applied to nodes)

quality assurance

quality control

Threat Ensemble Vulnerability Assessment and Sensor Placement Optimization Toolkit (name of software) 
xxii

Acronyms and Abbreviations 


\section{Acknowledgments}

\section{Authors}

The authors of this report are:

Michael J. Davis

Environmental Science Division

Argonne National Laboratory

Argonne, Illinois

Robert Janke

National Homeland Security Research Center

U.S. Environmental Protection Agency

Cincinnati, Ohio

Thomas N. Taxon

Decision and Information Sciences Division

Argonne National Laboratory

Argonne, Illinois

\section{Reviewers}

The following individuals reviewed a draft version of this report:

Argonne National Laboratory, Environmental Science Division, Argonne, Illinois Kurt Picel

U.S. Army Corps of Engineers, Engineering Research Development Center, Construction Engineering Research Laboratory, Champaign, Illinois

Jennie Feliciano

Mark Ginsberg

Kathryn Guy

Page Martin 
U.S. Environmental Protection Agency, National Homeland Security Research Center, Cincinnati, Ohio

Terra Haxton

Steve Klosterman

Matthew Magnuson

Jeff Szabo

University of Cincinnati, Department of Civil and Environmental Engineering, Ohio Ernesto Arandia 


\section{Summary}

An understanding of the nature of the adverse effects that could be associated with contamination events in water distribution systems is necessary for carrying out vulnerability analyses and designing contamination warning systems. This study examines the adverse effects of contamination events using models for 12 actual water systems that serve populations ranging from about $10^{4}$ to over $10^{6}$ persons.

The measure of adverse effects that we use is the number of people who are exposed to a contaminant above some dose level due to ingestion of contaminated tap water. For this study the number of such people defines the impact associated with an event. We consider a wide range of dose levels in order to accommodate a wide range of potential contaminants. For a particular contaminant, dose level can be related to a health effects level. For example, a dose level could correspond to the median lethal dose, i.e., the dose that would be fatal to $50 \%$ of the exposed population. The dose level required to reach a common endpoint can vary by orders of magnitude, depending on the toxicity of the contaminant. Highly toxic contaminants may be associated with a particular response at a very low dose level, whereas contaminants with low toxicity may only be associated with the same response at a much higher dose level.

This report focuses on the sensitivity of impacts to five factors that either define the nature of a contamination event or involve assumptions that are used in assessing exposure to the contaminant: (1) duration of contaminant injection, (2) time of contaminant injection, (3) quantity or mass of contaminant injected, (4) population distribution in the water distribution system, and (5) the ingestion pattern of the potentially exposed population. For each of these factors, the sensitivities of impacts to injection location and contaminant toxicity are also examined.

The sensitivity of impacts to the various factors studied is determined by comparing the impacts associated with different cases of the factor (e.g., 1-h versus 24-h injection durations) for injections at all non-zero demand nodes (i.e., pipe junctions where water is consumed) in a network. For the networks considered in this report, such comparisons involve simulation of injections at thousands of nodes. (Generally, the demand nodes examined in this report represent groups of service connections.) In order to facilitate comparisons, we identify locations of contaminant injection (network model nodes) in terms of the ranking of the associated impact. The $n^{\text {th }}$ percentile injection node is the node associated with the $n^{\text {th }}$ percentile impact. We examine two types of sensitivities to the various factors: sensitivity that results in variations in the magnitude of the $n^{\text {th }}$ percentile impact and sensitivity that results in changes in the injection locations that are associated with the $n^{\text {th }}$ percentile and higher impacts.

Impacts are sensitive to all the factors examined and the degree of sensitivity is dependent on the particular water system, the location of contaminant injection, and the dose level. For all the factors considered, sensitivity tends to increase with dose level (i.e., decreasing toxicity) of the contaminant, with considerable inter-network variability. With the exception of the population 
distribution (factor 4 above), sensitivity to the various factors tends to be highest at lower impact levels (e.g., impacts below the $80^{\text {th }}$ percentile). Conversely, for the population distribution factor, sensitivity is lowest at the lower impact levels. For injection duration, impacts generally are higher for longer duration injections. Definite patterns are present in the sensitivity of impacts to injection time, but these vary substantially across the networks. As would be expected, impacts are larger for larger mass injections, but the sensitivity can vary dramatically depending on dose level and the network. Estimated impacts can be sensitive to assumptions about how population is distributed in a network, particularly at high impact levels and high dose levels, again with considerable variability across networks. Finally, impacts can be sensitive to assumptions about ingestion patterns in the potentially exposed population, with sensitivities varying across networks and tending to be highest for high dose levels.

When considered in combination with the other factors (but not including the ingestion model used), impacts at low dose levels (levels at which the effects of highly toxic contaminants can be significant) are most sensitive to injection duration. Similarly, when considered in combination, impacts at higher dose levels (levels required for significant effects from contaminants with low toxicity) are most sensitive to injection mass. At low dose levels, for a likely range in injection masses, impacts are not particularly sensitive to injection mass.

The influence of the various factors on the location of high percentile injection locations can be as important or more important than their influence on the magnitudes of impacts. In addition, the choice of contaminant has a major influence on which nodes are high impact injection locations. The sharing (overlap) of the same high-percentile injection nodes for different values of a factor can vary substantially by contaminant and impact level (percentile of impact). Overlap tends to decrease with decreasing toxicity of the contaminant and increasing impact level for all the factors considered, with considerable variability among the networks.

Our results demonstrate the great variability in the impacts and the sensitivity of impacts to various factors that can occur in different water systems during a contamination event. Although definite patterns exist in the nature and magnitude of impacts and sensitivities for the diverse set of water systems examined, these results also show that substantial inter-network variability limits the ability to predict or extrapolate these results to other systems. Therefore, although water systems do exhibit some similarities in the magnitude and pattern of impacts during contamination events, each individual water system should be treated as unique. 


\section{Section 1}

\section{Introduction}

Contamination warning systems (CWSs) for water distribution systems need to be designed with a clear understanding of two issues. First, how are the adverse effects associated with contamination events influenced by the major factors that define the contamination event? Second, how might these effects vary for a wide range of distribution systems and contaminants? In particular, the sensitivity of estimated responses to these factors and to the major assumptions used in estimating the responses needs to be understood. Various studies have examined potential adverse effects associated with contamination events, generally for small water distribution systems (Khanal 2005; Nilsson et al. 2005; Khanal et al. 2006; Davis and Janke 2008). Using a simple network with less than 90 nodes (pipe junctions), Khanal (2005) and Khanal et al. (2006) examined the sensitivity to selected network variables of the potential for exposure to contaminants. They determined a potential for exposure but considered only the presence of a contaminant at network nodes, not the estimated actual exposures of consumers of contaminated water. No study has examined the sensitivity of potential exposures to contaminants to major factors used in the simulation considering the actual exposure process and systems of significant size (i.e., thousands of nodes). Separately, we have analyzed potential exposures associated with contamination events using 12 models of actual water systems serving populations ranging from about $10^{4}$ to over $10^{6}$ persons, while considering the exposure process (Davis and Janke 2010). Using these same models and extending that work, this report examines the sensitivity of potential exposures to (1) a number of major factors that define the nature of a contamination event and (2) assumptions that are used in assessing exposure to the contaminant. The results presented here should be useful to those analyzing adverse effects associated with contamination events in water distribution systems and to designers of CWSs.

\subsection{Definitions}

The following terms are used throughout this report with the meanings given here.

Dose. A dose is the quantity of a contaminant ingested by consumption of tap water. Inhalation and dermal contact may also be exposure pathways, but for most contaminants of interest, ingestion is the most important. Ingestion doses usually are expressed as a mass of contaminant per unit of body weight (i.e., in units of $\mathrm{mg} / \mathrm{kg}$ ). In this report doses are expressed as contaminant mass ingested (mg). Assuming a typical body weight of $70 \mathrm{~kg}$, doses in units of $\mathrm{mg} / \mathrm{kg}$ can be obtained from the doses reported here by dividing results by $70 \mathrm{~kg}$.

Dose Level. A dose level is simply some level of dose. For a particular contaminant, it can 
be related to a health effects level. For example, a dose level could correspond to the median lethal dose. The dose level required to reach a common endpoint can vary by orders of magnitude, depending on the contaminant. Therefore, we consider a wide range of dose levels in order to accommodate the potentially wide variability in responses to different contaminants.

Impact. Impact is the size of the population exposed to a contaminant above some dose level by ingestion of contaminated tap water. It is the measure of adverse effects associated with a contamination event that we use in this report. Impact could be defined as the number of fatalities or illnesses that result from exposure to contaminated water. However, to reduce the complexity of the analysis, the focus is on contaminant doses and the major exposure pathway.

\subsection{Navigating This Report}

The methodology used in this report is presented in Section 2. Section 3 discusses the nature of the impacts considered. Results are presented in Sections 4 through 9 and discussed in Sections 10 and 11. Major conclusions are summarized in Section 12. Finally, the appendix describes the approach to quality assurance used in this study.

The electronic version of this report contains various links that can be used to move from one location to another. All references to a figure, table, or document have links to the actual figure, table, or document description. All entries in the Table of Contents and lists of figures and tables contain links. In Acrobat Reader ${ }^{\circledR}$, using the "Previous View" button will return the user to the original reference. If the button is not installed, it can be installed by going to Tools on the Reader menu bar and then going to Customize Toolbars. 


\section{Section 2}

\section{Methodology}

We simulated impacts to consumers of contaminated tap water during a contamination event for a number of water distribution systems and examined the sensitivity of the estimated impacts to major factors characterizing the injection of contaminant, the distribution of population in the system, and the ingestion of contaminated water. By simulating diverse distribution systems, it is possible to determine how sensitivities vary across systems and identify the factors for which estimated impacts are sensitive to the network used and those for which the sensitivitiess are generally independent of the network.

\subsection{Water Distribution Systems Examined}

The systems and associated network models used are described in Table 2.1. The systems modeled are all real systems, but not all of the network models used represent complete systems. (The networks in some cases may represent incomplete systems but are always complete with respect to the area being represented.) For security reasons, we do not identify the systems or provide their general location. However, some of the network models have been used previously. Watson et al. (2009) used our Networks 1 and 4 (their Networks 1 and 2) and Ostfeld et al. (2008) used our Network 6 (their Network 2) for sensor monitoring research and evaluation. Isovitsch and VanBriesen (2008) used our Network 6 in a study of optimal sensor placement. Murray et al. (2009) examined the influence of district metering areas on a number of factors using case studies, including two networks that are the same as our Networks 4 and 6.

The populations served by the systems range in size from less than $10^{4}$ to over $10^{6}$ and the population density varies from about $10^{2}$ to over $10^{4}$ persons $/ \mathrm{km}^{2}$. Figure 2.1 shows the distribution of population at network nodes that have non-zero demands (i.e., nodes where water is consumed) for nodes with up to 250 people per node, assuming that population is proportional to nodal demand. (Generally, the nodes examined in this report represent groups of service connections.) Maximum nodal populations are given in Table 2.1, again assuming population is proportional to demand. Some portion of the differences in population distribution is due to differences in the degree of skeletonization of the networks. Four of the network models (Networks 2, 3, 6, and 10) are considered 'all pipe' models because of the pipe detail per service connection included in the models. These networks have the smallest mean populations per non-zero demand (NZD) node (Table 2.1). Average daily water demand for the networks ranges from about 60 to over $9000 \mathrm{~L} / \mathrm{s}$ (1-200 million gal/d). Analysis with EPANET (Rossman 2000), software for simulating the hydraulic and water 
Table 2.1. System Descriptions ${ }^{\dagger}$

\begin{tabular}{crrrrrrrrr}
\hline & & & Mean pop. & \multicolumn{2}{c}{ Mean } & \multicolumn{3}{c}{ Non-zero demand nodes } \\
\cline { 7 - 10 } Network & Pop. & Area & $\begin{array}{r}\text { density } \\
\left(10^{3}\right)\end{array}$ & water & No. of & & Mean & Median & Max. \\
& $\left(\mathrm{km}^{2}\right)$ & $\left(\right.$ no./km $\left.{ }^{2}\right)$ & age $(\mathrm{h})$ & nodes & No. & pop. & pop. & pop. \\
\hline 1 & 6 & 40 & 160 & 68 & 410 & 110 & 61 & 15 & 1,400 \\
2 & 75 & 78 & 1,000 & 17 & 3,200 & 3,000 & 25 & 17 & 1,000 \\
3 & 130 & 130 & 1,000 & 45 & 6,800 & 6,700 & 20 & 13 & 4,300 \\
4 & 150 & 500 & 310 & 50 & 3,400 & 1,600 & 94 & 76 & 1,400 \\
5 & 190 & 260 & 740 & 24 & 3,000 & 1,800 & 100 & 58 & 9,000 \\
6 & 250 & 490 & 520 & 26 & 13,000 & 11,000 & 24 & 16 & 3,200 \\
7 & 590 & 21 & 28,000 & 7 & 4,400 & 2,200 & 260 & 110 & 18,000 \\
8 & 790 & 2,700 & 290 & 30 & 7,400 & 5,900 & 130 & 83 & 2,000 \\
9 & 840 & 1,600 & 520 & 38 & 8,100 & 6,800 & 120 & 75 & 60,000 \\
10 & 1,500 & 1,200 & 1,200 & 160 & 43,000 & 28,000 & 51 & 12 & 73,000 \\
11 & 1,500 & 1,400 & 1,100 & 110 & 14,000 & 8,700 & 170 & 53 & 110,000 \\
12 & 1,800 & 3,700 & 480 & 79 & 3,100 & 1,400 & 1,200 & 790 & 16,000 \\
\hline
\end{tabular}

$\dagger$ The systems are listed by population (pop.) size. All numbers (no.) are rounded independently to two significant figures.

quality behavior of networks of pressurized pipes, yields mean water ages (i.e., residence times) ranging from 7 to $160 \mathrm{~h}$.

We consider a diverse set of distribution systems, as summarized in Table 2.1. In addition, some of the systems are supplied primarily by surface water, while a few depend almost entirely on ground-water sources. Tanks dominate some systems as a result of significant elevation obstacles. Other systems are in areas with little elevation change. For the systems dominated by groundwater sources, the sources are spatially diverse, potentially making those systems less vulnerable to widespread contamination.

Maps of the network models for Networks 4 and 6 are shown in Figures 2.2 and 2.3, respectively. The figures show distorted versions of the actual networks. For security reasons we do not provide maps for any additional networks.

The systems used in this study were selected because of the availability of complete network models and a desire to include a wide range of system sizes. The systems are not a random sample of water distribution systems. However, a broad range of networks is included and therefore our conclusions should be relevant to a wide range of applications.

\subsection{Analysis}

To examine the sensitivity of impacts to various factors, we defined a number of cases involving these factors. For each network and case considered, we simulated the following: (1) the independent injection of a contaminant at each of the NZD nodes in the network, (2) transport of the contaminant in the network following injection, and (3) exposure to the contaminant by ingestion 


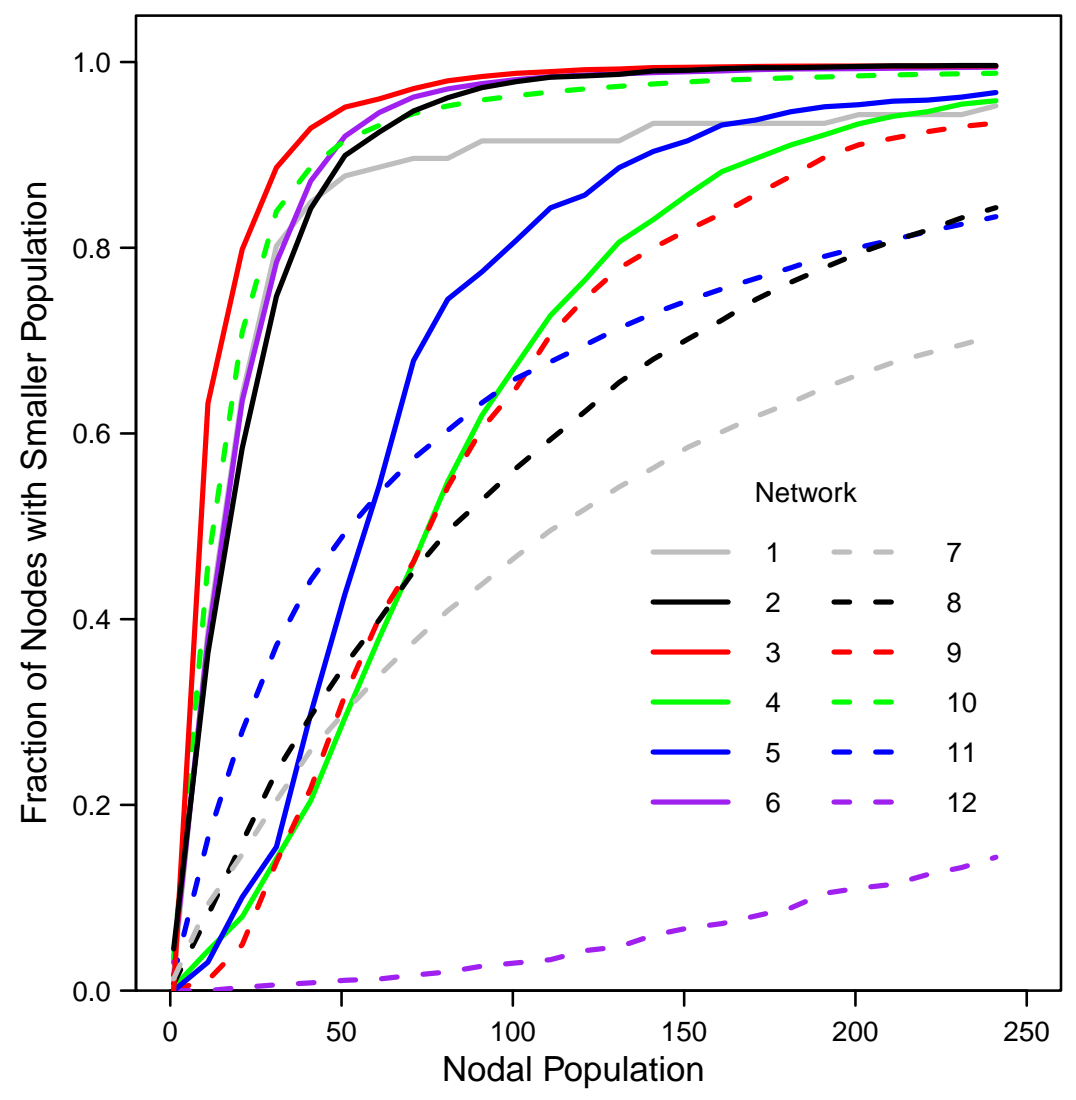

Figure 2.1. Distribution of nodal populations for the networks below 250 persons per node. 


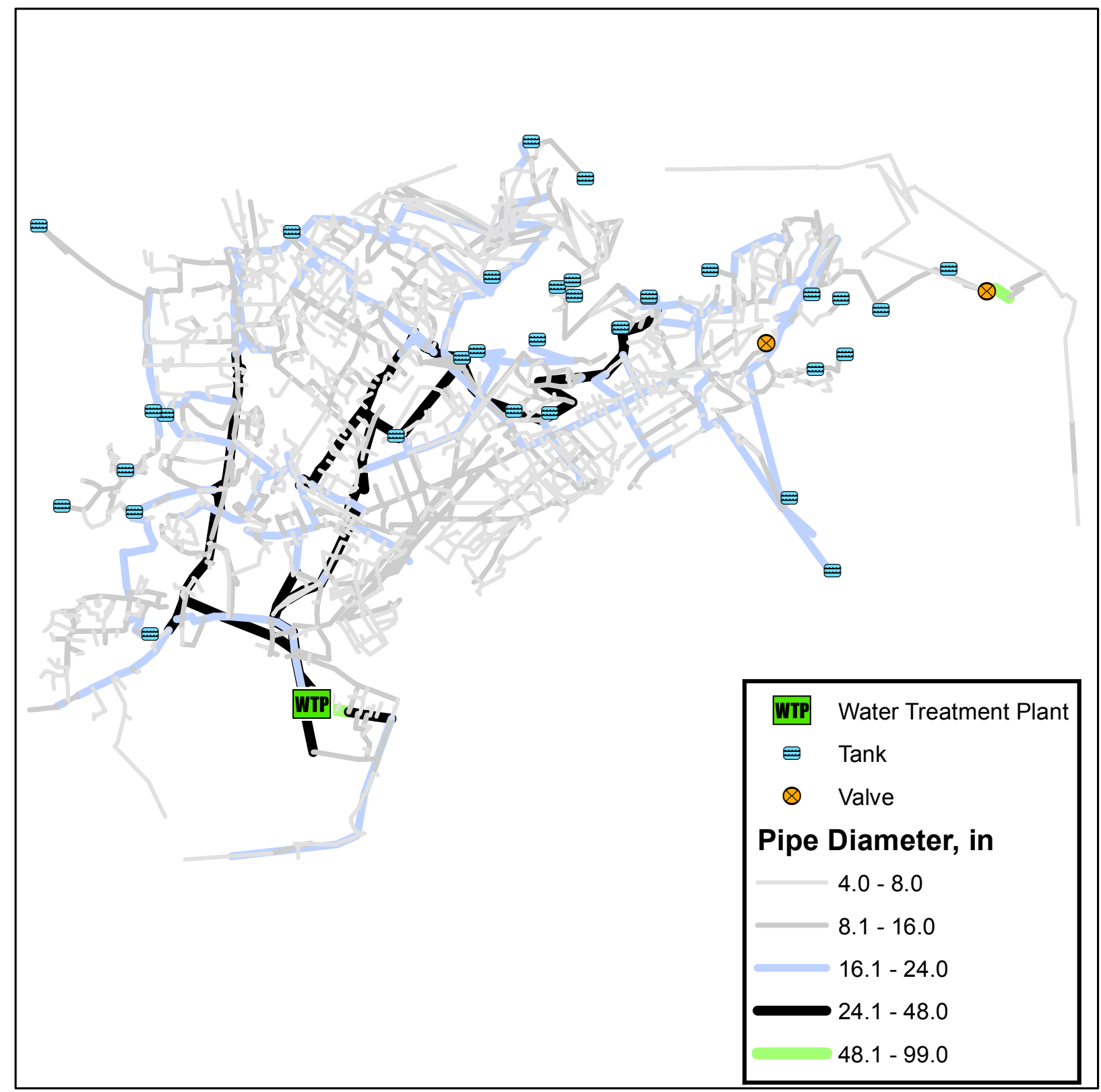

Figure 2.2. Network 4 . 


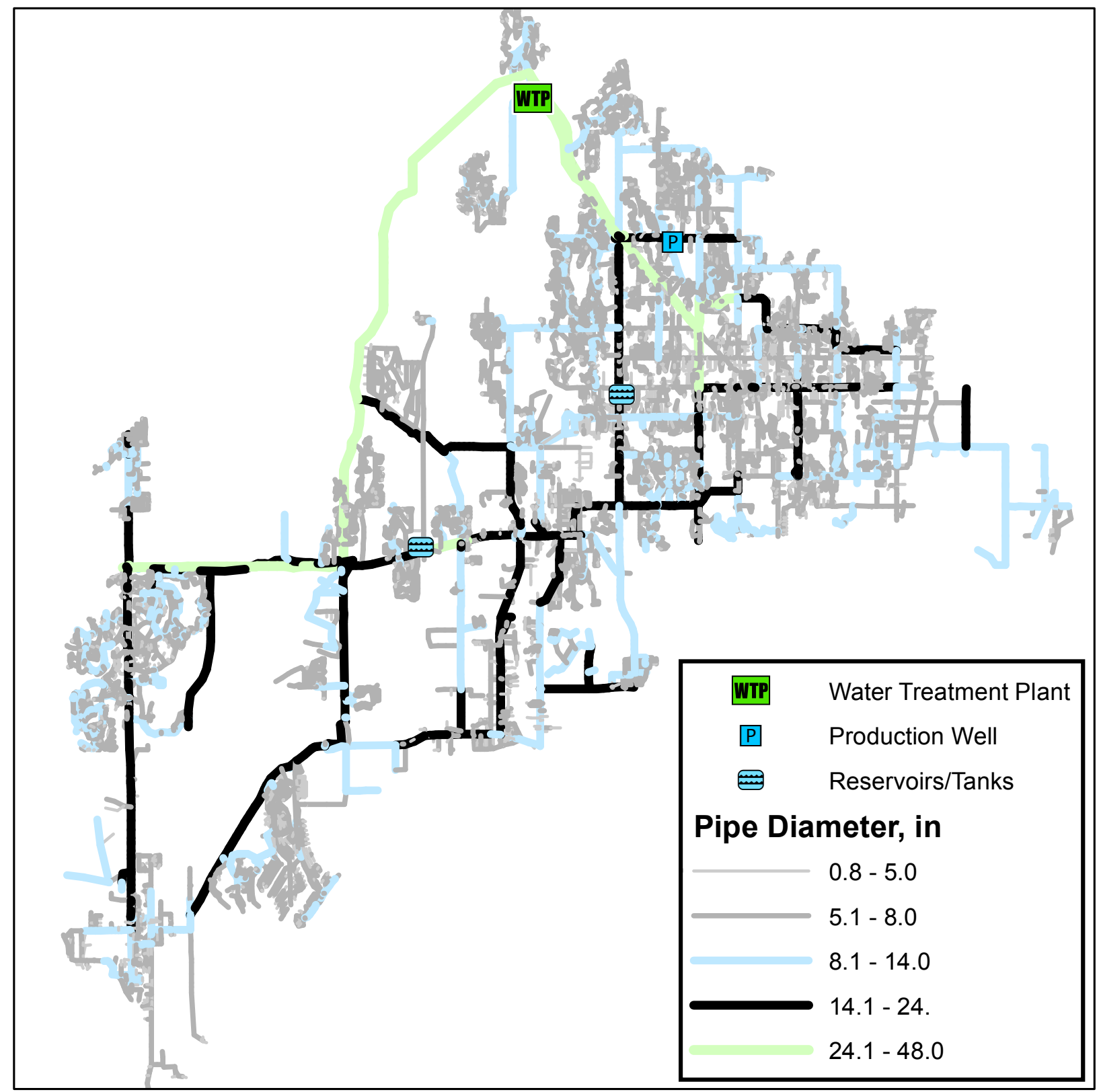

Figure 2.3. Network 6. 
of tap water from the network. As an example, for a particular case involving Network 11 we did 8,700 independent simulations, one for each NZD node. Each of these simulations represents one contamination event for the network, resulting from an injection at a particular node.

NZD nodes generally represent a number of service connections. We used them in our analysis because they represent locations where injections are most likely to occur. Zero demand nodes, such as nodes corresponding to fire hydrants, could also be potential injection locations. However, such locations are more likely to be inaccessible than NZD nodes or, in the case of fire hydrants, such locations are not easily concealed.

The factors that define a contaminant injection are the time of day that the injection begins, the duration of the injection, and the mass of contaminant injected. Our baseline case is an injection beginning at 0:00 hours local time and lasting for one hour. We use a realistic injection mass that results in significant contaminant concentrations in the networks but we do not specify the mass in order to prevent the results in this report from being used for design purposes. In our sensitivity analysis we also consider injections with ten times the mass used for the baseline case, injections that begin at 06:00, 12:00, and 18:00 hrs, and injections that last for $24 \mathrm{~h}$.

We simulated contaminant transport in the water distribution systems with TEVA-SPOT sensor placement optimization software (U. S. EPA 2010a), which uses EPANET (version 2.00.12) as its primary simulation engine. Time steps for hydraulics were $1 \mathrm{~h}$ or less; time steps for water quality were $1 \mathrm{~min}$. Water quality tolerances were specified in the models obtained and were generally the EPANET default of $0.01 \mathrm{mg} / \mathrm{L}$. Contaminants were assumed to behave as conservative tracers following injection (i.e., they were not retarded or degraded in the system).

There are numerous parameters that describe a distribution system that must be specified for EPANET. We used the paramenters in the network models supplied by various utilities. However, although populations served by the systems are known, their distribution to NZD nodes is not. Therefore, to account for the sensitivity of results to how population is distributed in a network, we considered (1) population distributed in proportion to nodal demand and (2) equal population at all nodes. The total population for a system is the same for both cases. The baseline case is demand-based population.

We estimated ingestion doses for consumers of tap water using two different models. Ingestion of tapwater is likely to be the major exposure pathway for most contaminants. Ingestion dose depends on contaminant concentration in the water ingested and the volume of water ingested. Because contaminant concentration in tap water varies with time during a contamination event, ingestion times are needed in order to determine contaminant concentrations in ingested water. The ingestion models used are summarized in Table 2.2 and have been discussed previously (Davis and Janke 2008). Both of the ingestion models have a component that addresses ingestion timing and a component that addresses the volume of tap water consumed. Model 1 is our baseline case. It uses a probabilistic approach to determining both ingestion times and volume. Model 2 is a completely deterministic model that uses an average ingestion volume but assumes ingestion throughout the day in amounts proportional to nodal demand.

Repeated simulations with Model 1 produce different results. It would be preferable to carry out multiple simultations and obtain mean results. However, use of such an approach has excessive computational requirements. Variability in impacts for different simulations with the model is relatively small; the relative standard error in impacts obtained for multiple simulations for Network 4 is about $2 \%$ or less (Davis and Janke 2010). Consequently, a single simulation with the model provides results that are acceptable for this study. 
Table 2.2. Ingestion Models

\begin{tabular}{cll}
\hline Model & Timing Component & Volume Component \\
\hline 1 & $\begin{array}{l}\text { Ingestion based, probabilistic (5 } \\
\text { events per day), based on time-use } \\
\text { studies }\end{array}$ & $\begin{array}{l}\text { Variable daily volume (probabilistic), } \\
\text { based on U. S. EPA (2000a) }\end{array}$ \\
2 & $\begin{array}{l}\text { Demand based, every hour (24 } \\
\text { events per day), used in some } \\
\text { studies }\end{array}$ & $\begin{array}{l}\text { 1 L per day, based on mean volume } \\
\text { of tap water ingested per day }\end{array}$ \\
\hline
\end{tabular}

Table 2.3. Factors Considered in the Sensitivity Analysis

\begin{tabular}{lll}
\hline Factor & Cases Considered & Baseline Case \\
\hline Injection duration & 1 and 24 $\mathrm{h}$ & $1 \mathrm{~h}$ \\
Injection time & $0: 00,6: 00,12: 00$, and 18:00 & $0: 00$ \\
Injection mass & Realistic mass (1X) and & $1 \mathrm{X}$ \\
& ten times larger mass (10X) & \\
Population distribution & Demand-based at each node and & Demand-based at each node \\
& average value at each node & \\
Ingestion model & Models 1 and 2 & Model 1 \\
\hline
\end{tabular}

We do not include children in our analysis. Also, we examine only the sensitivity of results to various factors that do not include the ages of exposed individuals.

Table 2.3 summarizes the factors examined in the sensitivity analysis and the variations considered in the factors. We also considered combinations of factors to examine interactions. The first case shown for each factor in Table 2.3 is the baseline case. Although estimated impacts may be sensitive to uncertainties in the network models used, we did not examine sensitivity to any parameters or details of the network models.

In our analysis we compare impacts that result from the injection of a contaminant at a particular node for various cases to determine the sensitivity to different factors, such as injection time. To allow such comparisons to be made, we need a method to identify nodes by the ranking of the associated impact, in addition to simply using the name of a particular node. Therefore, we define the $n^{\text {th }}$ percentile injection node as the injection node associated with the $n^{\text {th }}$ percentile impact. Nodes corresponding to a particular percentile impact generally vary with the dose level used to define the impact. Therefore, when identifying a node, we specify both its rank and the dose level used to define the impact. For example, we might refer to a node as the $90^{\text {th }}$ percentile node for a dose level of $10 \mathrm{mg}$.

As indicated in the previous paragraph, when factors change, the impact associated with an injection at a particular node can change. These changes can influence the importance of the impact of an injection at a particular node relative to impacts of injections at all other NZD nodes 

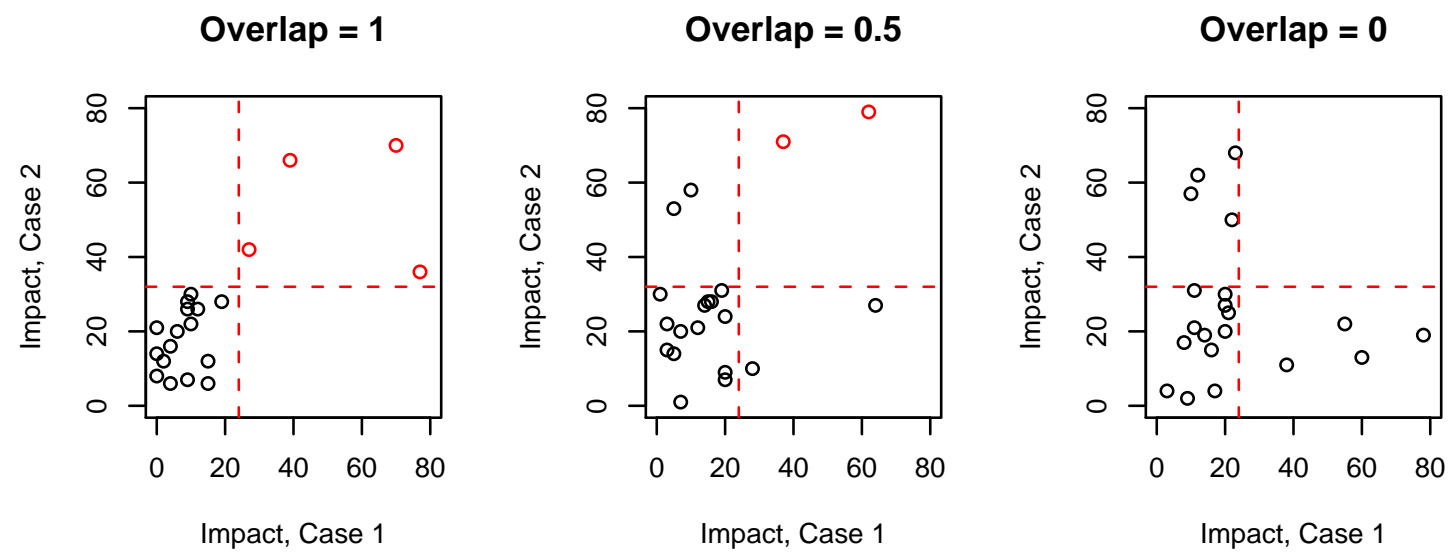

Figure 2.4. Overlap of high-percentile injection nodes for a hypothetical network with 20 nodes and two cases of some factor. The horizontal dashed lines give the $80^{\text {th }}$ percentile impact for Case 2 and the vertical dashed lines give the $80^{\text {th }}$ percentile impact for Case 1 . Red points correspond to nodes that are associated with impacts that are above the $80^{\text {th }}$ percentile for both cases.

for the network. To quantify the consistency of the importance of nodes as injection sites, we define an overlap between injection nodes that are associated with impacts at or above some percentile for one case of a factor (Case 1) and the injection nodes associated with impacts at or above the same percentile for another case of a factor (Case 2). Overlap is simply the fraction of nodes possibly having a particular percentile rank or higher rank that actually have that rank or a higher rank for both cases. Our interest is in the overlap of high-percentile injection nodes for different values of some factor.

The concept of overlap of high-percentile injection nodes is illustrated in Figure 2.4, which provides scatter plots comparing impacts for two hypothetical cases of some factor for a network with 20 nodes. The plots show examples of overlaps equal to $1,0.5$, and 0 for $80^{\text {th }}$ percentile and higher impacts for some dose level. Each of the 20 points in the plots corresponds to an injection node. The red dashed lines give the $80^{\text {th }}$ percentile impacts. For each case there are four nodes that are associated with impacts at or above the $80^{\text {th }}$ percentile. For an overlap of 1 , the same four nodes are associated with $80^{\text {th }}$ percentile or higher impacts for both cases. (Note that although the percentile is the same for both cases, the associated impact is not the same for the two cases.) For an overlap of zero, no node is associated with $80^{\text {th }}$ percentile or greater impacts for both cases. For an overlap of 0.5 , two injection nodes are associated with impacts that have a percentile rank greater than 80 for both cases.

Overlap can be expressed as:

$$
\text { overlap }=\frac{N_{p}}{\left(1-\frac{P}{100}\right) N}
$$

where

$N=$ number of injection nodes (i.e., NZD nodes), 
$P=$ the percentile of the impact of interest, and

$N_{P}=$ number of injection nodes associated with impacts $\geq P^{t h}$ percentile impacts, both cases. When overlap $=1$, all injection nodes for Case 1 that are associated with impacts at or above the given percentile are also associated with impacts at or above that percentile for Case 2 . If $N=20$ and $P=80 \%$, then $N_{P}$ would be 4 for overlap $=1$, as illustrated in Figure 2.4. When overlap $=0$, there are no injection nodes that are associated with impacts at or above the given percentile for both cases. When a particular percentile impact is zero, all injection nodes are associated with impacts that are at or above that impact. In such a case the overlap calculated will be 1 . Therefore, when the impact associated with the percentile of interest for one or both of the cases is zero, no overlap is determined.

The analysis of overlap shows that the locations of high-percentile injection nodes varies when the factors studied are changed. However, the analysis does not provide any information on changes in the actual spatial distribution of the nodes as the factors change. To show the actual spatial changes in the locations of high impact injection nodes, we provide maps for selected networks with the actual locations of injection nodes for different values of the various factors studied.

To display distributions of impacts for particular cases for a given network, we use violin plots (Hintze and Nelson 1998). Figure 2.5 provides a comparison between a violin plot and a box plot. Violin plots combine the information provided by a box plot with additional information on the shape of the distribution. Box plots cannot be used to infer the shape of the distribution of values of the quantity being examined. However, violin plots can be used for that purpose.

Sensitivity of impacts to changes in a factor is examined in two different ways. First, the influence of the factor on the statistics of the impacts is considered. For example, how does changing the injection duration affect the magnitude of the $95^{\text {th }}$ percentile impact for a particular dose level? Second, the influence of the factor on impacts at particular nodes is examined. For example, again considering injection duration, how does changing this factor affect the degree of overlap of high percentile nodes at a particular dose level?

We also examine the sensitivity of impacts to combinations of factors. Estimating impacts for a large combination of these factors becomes computationally challenging. Considering injection time, duration, and mass as continuous quantities and considering two population distributions and two dose models could result in many thousands of sets of simulations for a single network if a Monte Carlo analysis were used to examine impacts for a wide range of combinations of factors. Each set of simulations could involve thousands of individual simulations for each NZD node when using the probabilistic exposure model. To limit the size of the problem, we consider four injection times (0:00, 6:00, 12:00, and 18:00), two injection durations ( 0 and $24 \mathrm{~h}$ ), two injection masses, and two population distributions (average value at each node and demand based at each node), yielding 32 combinations of factors. We do not include ingestion models as a factor because doing so would double the number of combinations. Using the set of 32 combinations of factors for one network, we performed one simulation for each combination for each NZD node for the network. We then examined the patterns in the estimated impacts to determine which factors, when considered together, have a major influence on the impacts, as will be discussed in Section 9.

Sections 4 through 8 consider the sensitivity of impacts to a number of factors: injection duration, injection time, injection mass, the distribution of the population in the network, and the model used to estimate ingestion dose. Each section considers one of the factors at a time. Combinations of factors are then considered together in Section 9. In the sections considering the individual 


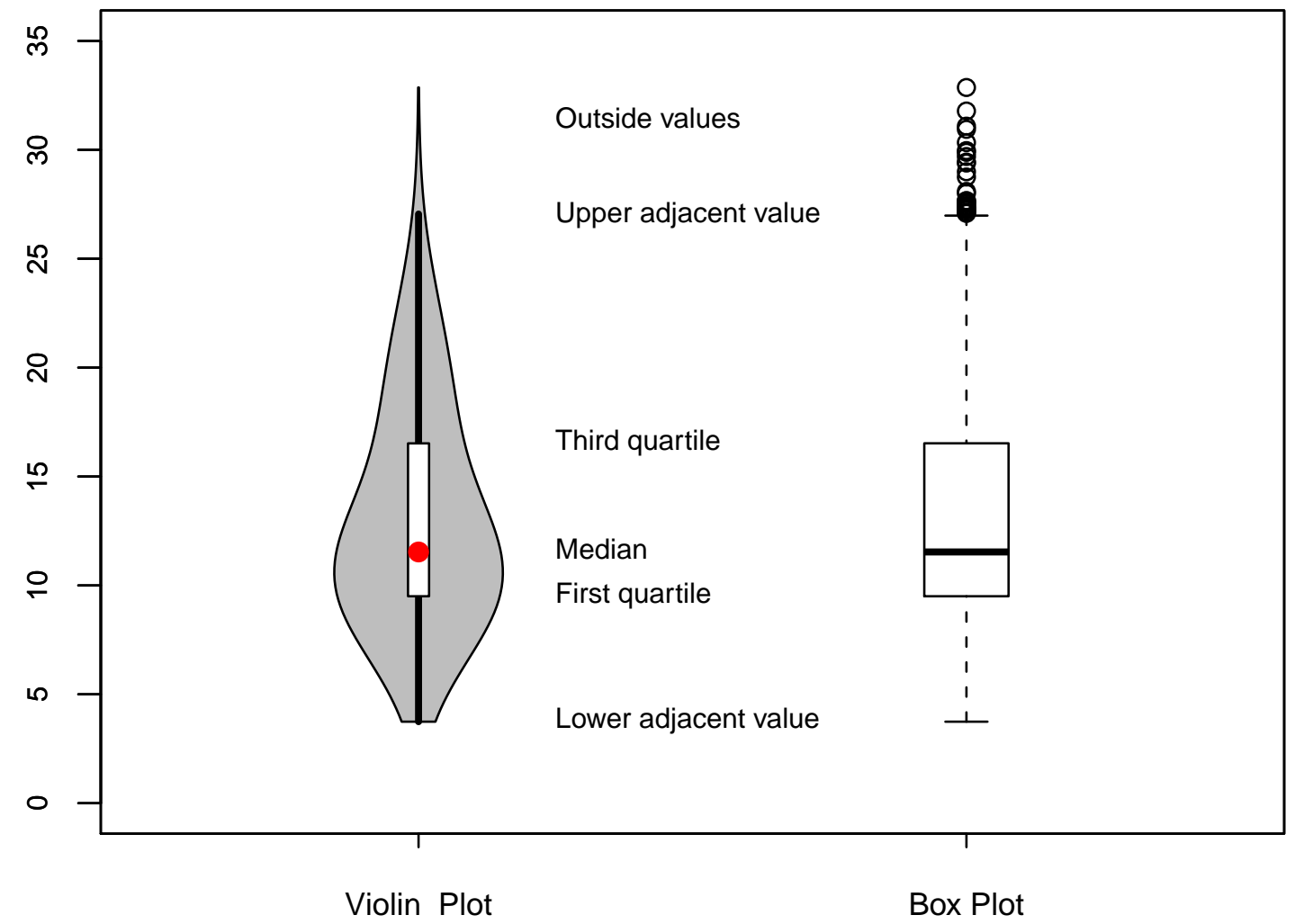

Figure 2.5. Comparison of a violin plot and a box plot. 
factors, results are presented in a series of seven types of figures that are repeated for each factor. In addition, maps are used to illustrate how impacts vary spatially as the factors are varied. The various figures and maps are discussed in Section 4 as each one is introduced. 


\section{Section 3}

\section{Nature of Impacts}

As already noted, in this study impact is defined as the size of the population exposed above some dose level due to ingestion of contaminated tap water. This section presents some basic information about impacts.

Figures 3.1, 3.2, and 3.3 show how impacts vary with dose level for the $50^{\text {th }}, 75^{\text {th }}, 85^{\text {th }}, 95^{\text {th }}$, $99^{\text {th }}$, and $100^{\text {th }}$ percentile injection nodes for the 12 networks ${ }^{1}$. (Note the logarithmic scales on the horizontal and vertical axes.) The behavior of impacts is generally consistent for all the networks. First, impact decreases with dose level. This decrease occurs because fewer people receive high doses than low doses and impact is defined as the number of people exposed above a particular level. Second, for any dose level, impact increases with the increasing percentile of the injection node. However, for some networks, such as Network 5, the impacts vary much less between the median and maximum percentile nodes than for others, such as Network 11. Because significant impacts, those similar in magnitude to worst-case impacts, are associated only with the high percentile cases, we focus our attention here on injections at high percentile nodes.

The results in Figures 3.1, 3.2, and 3.3 (and other figures) are not specific to any contaminant but can be applied to specific contaminants. For example, the median lethal dose for ingestion of botulinum toxin is about $10^{-6} \mathrm{mg} / \mathrm{kg}$, or about $10^{-4} \mathrm{mg}$ for a $70-\mathrm{kg}$ person, a value at the lower end of the range of dose levels used in Figures 3.1, 3.2, and 3.3. The upper end of the range of dose levels in Figures 3.1, 3.2, and 3.3 is $100 \mathrm{mg}$, or about $1 \mathrm{mg} / \mathrm{kg}$ for a $70-\mathrm{kg}$ person, a value similar to the median lethal dose for phorate, a pesticide. The use of a wide range in dose levels allows us to consider the equivalent of a wide range in potential contaminants.

For higher dose levels, impacts may be zero for some cases. In such cases, no points are shown in Figures 3.1, 3.2, and 3.3 or similar figures. For example, for Network 5 in Figure 3.2, no $50^{\text {th }}$ percentile impacts are shown for dose levels of 10 and $100 \mathrm{mg}$ because they are zero.

As already noted, the injection node that is the $n^{\text {th }}$ percentile injection node for a particular dose level generally is not the $n^{\text {th }}$ percentile injection node for a different dose level. Figures such as Figures 3.1, 3.2, and 3.3 that show impacts for a particular impact level as a function of dose level are showing impacts associated with generally different injection nodes.

The spatial extent of the population of high percentile injection nodes can expand substantially as the percentile of interest decreases. Figures 3.4, 3.5, and 3.6 illustrate this behavior for Net-

\footnotetext{
${ }^{1}$ According to the standard definition of percentile, the $n^{\text {th }}$ percentile impact is the impact below which $n$ percent of the impacts lie. Therefore, there can be no $100^{\text {th }}$ percentile impact. However, to simplify graphical presentations in this report, we use $100^{\text {th }}$ percentile to mean maximum value.
} 


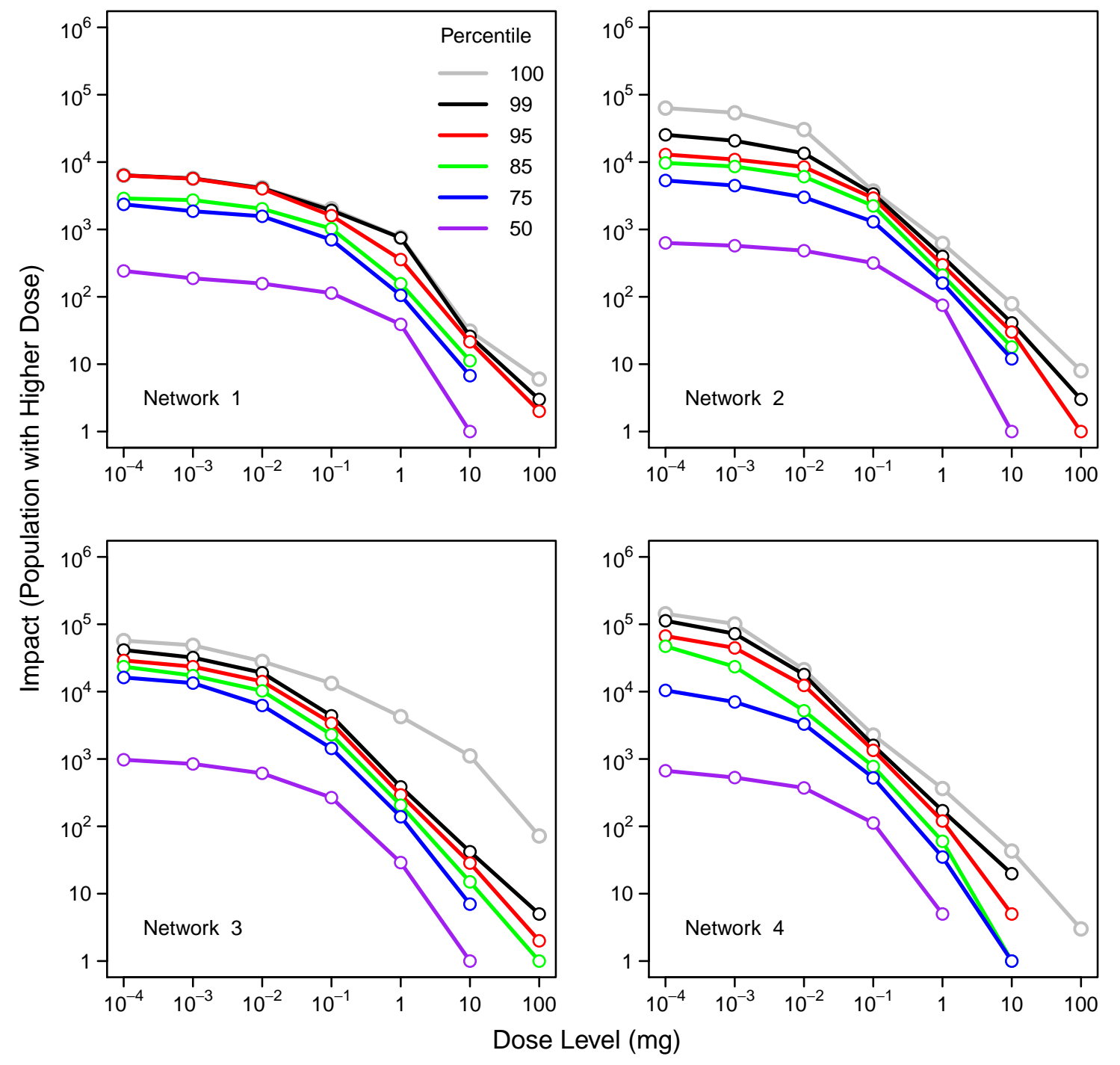

Figure 3.1. Impacts for Networks 1 to 4 as function of dose level, for various percentile injection nodes. 


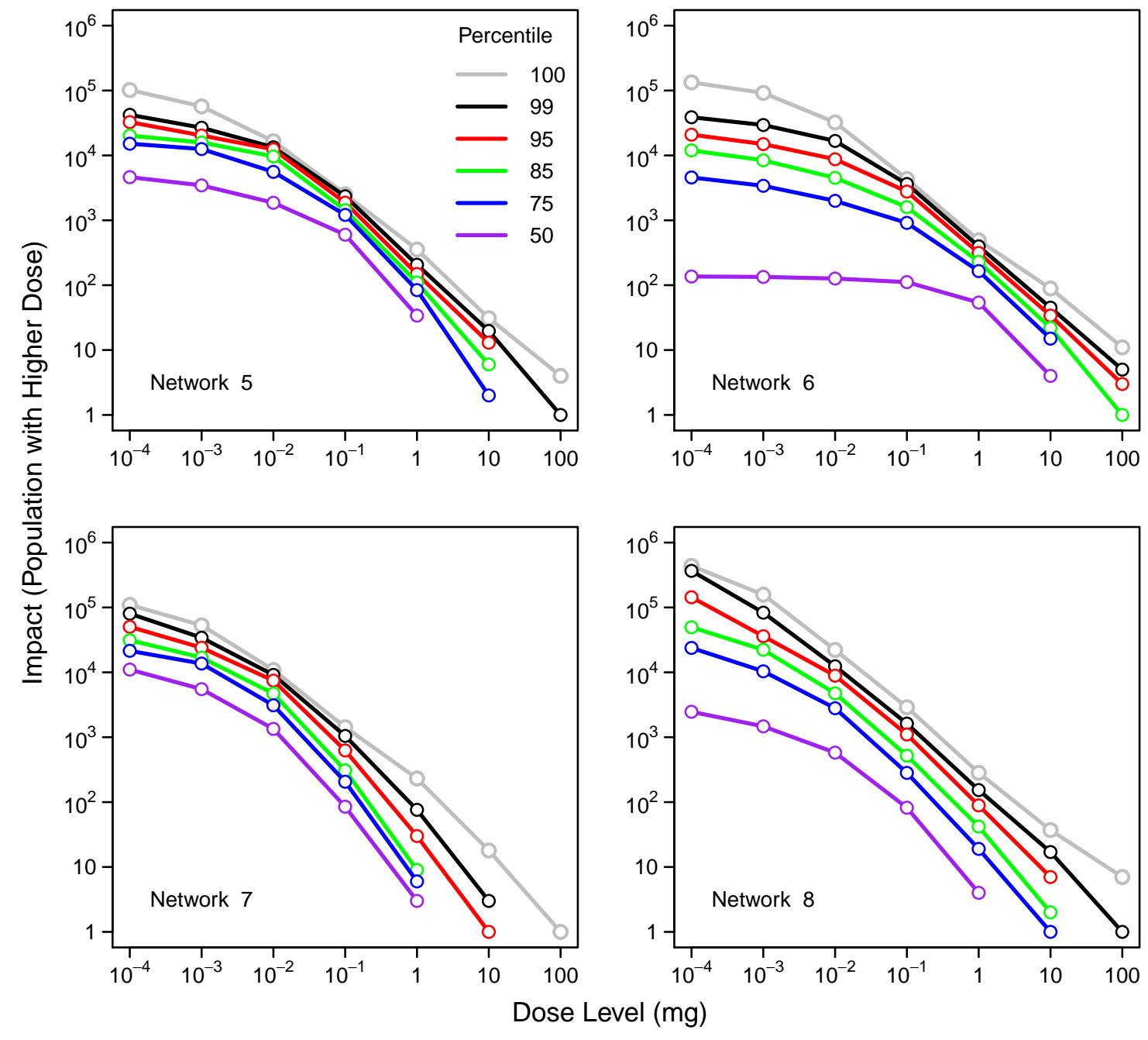

Figure 3.2. Impacts for Networks 5 to 8 as function of dose level, for various percentile injection nodes. 


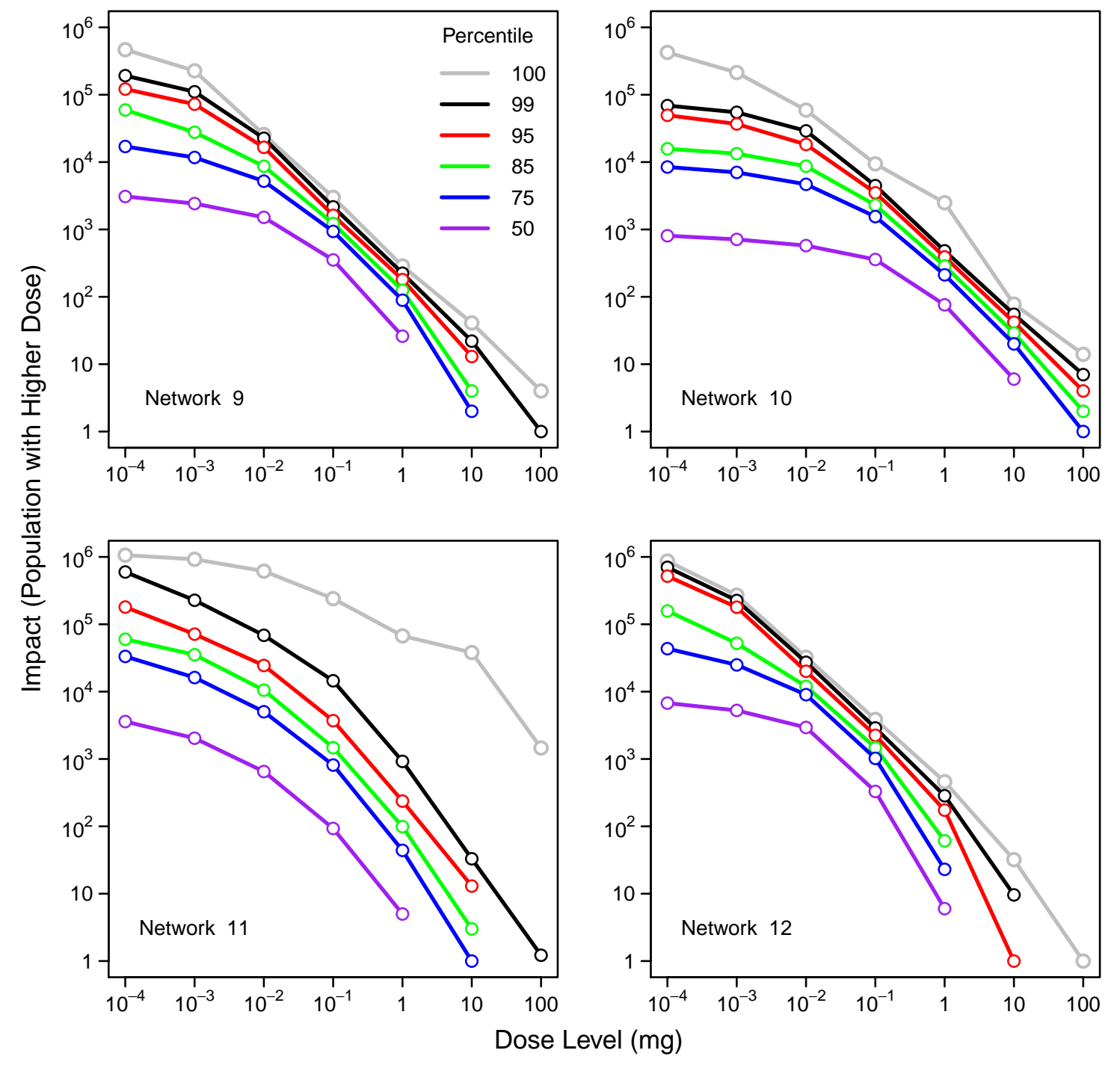

Figure 3.3. Impacts for Networks 9 to 12 as function of dose level, for various percentile injection nodes. 
work 4 for a dose level of $0.0001 \mathrm{mg}$ and the $95^{\text {th }}, 90^{\text {th }}, 85^{\text {th }}$, and $80^{\text {th }}$ percentiles. The maps in the figures show the locations of injection nodes associated with impacts at or above the percentile given.

The areas affected by injection at the $n^{\text {th }}$ percentile node for different dose levels can vary significantly depending on the dose levels of interest. Figure 3.7 provides an example for Network 6 showing the areas (receptor nodes) affected following injection at the $90^{\text {th }}$ percentile nodes for dose levels of 0.0001 and $1.0 \mathrm{mg}$. For the lower dose level the affected area is substantial and occupies a large portion of the central part of the network. For a dose level of $1.0 \mathrm{mg}$, the area affected involves only a relatively small number of nodes in the southwestern portion of the network. Figure 3.7 also illustrates that the location of a given percentile node can vary greatly with dose level. 


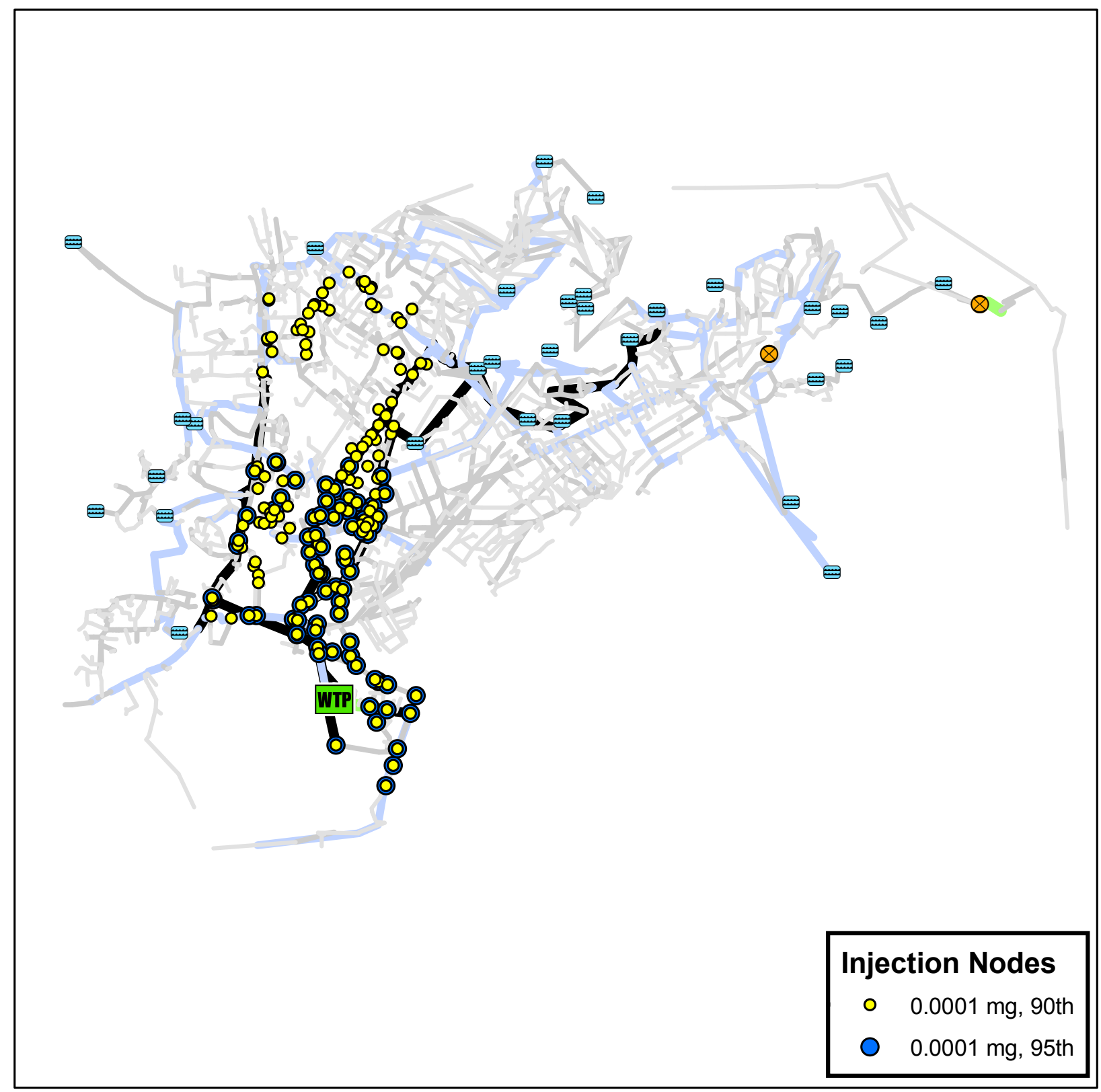

Figure 3.4. Network 4 showing locations of injection nodes associated with $90^{\text {th }}$ and $95^{\text {th }}$ percentile or higher impacts at a dose level of $0.0001 \mathrm{mg}$. Additional features on the map are identified in Figure 2.2. 


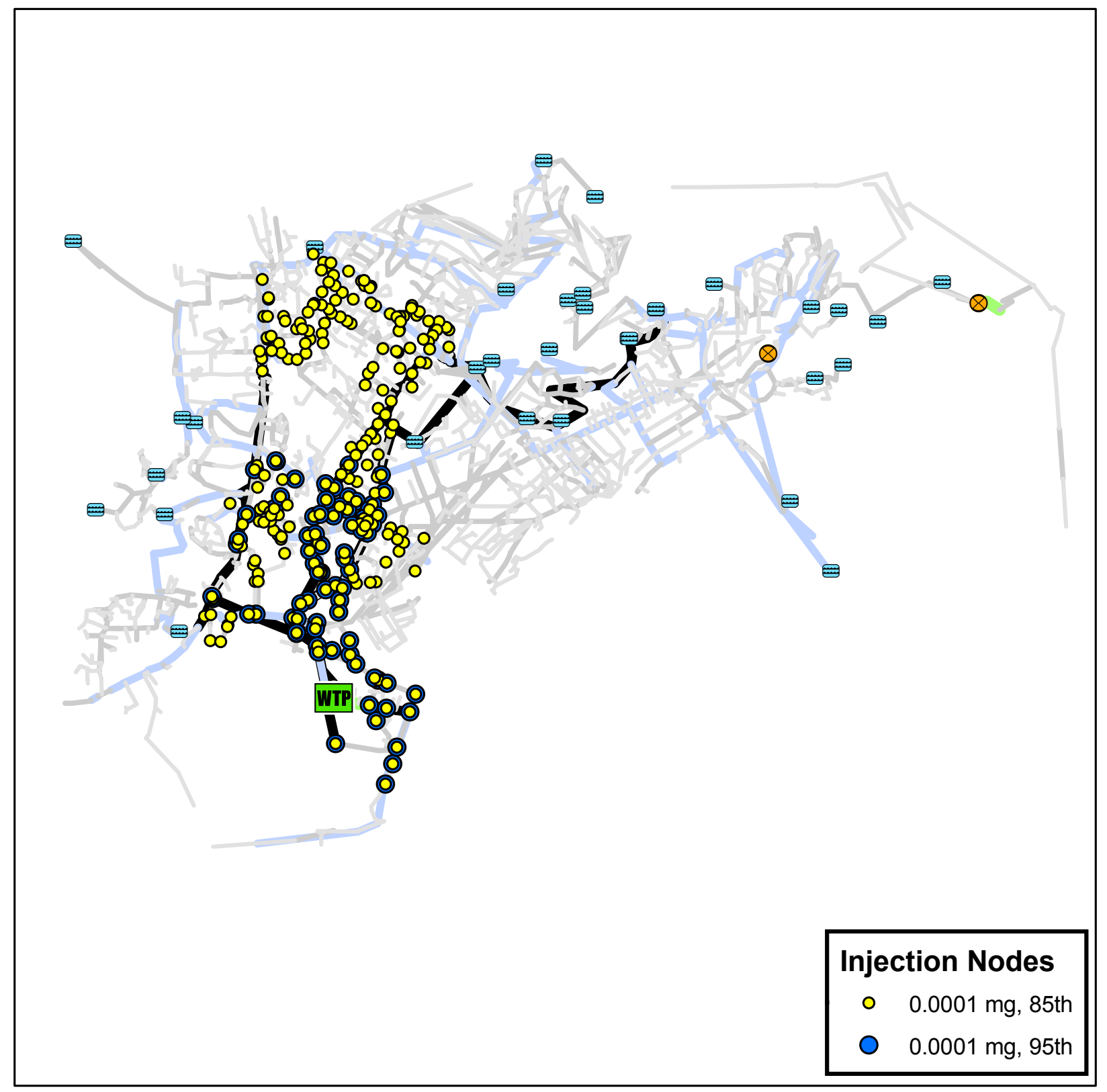

Figure 3.5. Network 4 showing locations of injection nodes associated with $85^{\text {th }}$ and $95^{\text {th }}$ percentile or higher impacts at a dose level of $0.0001 \mathrm{mg}$. 


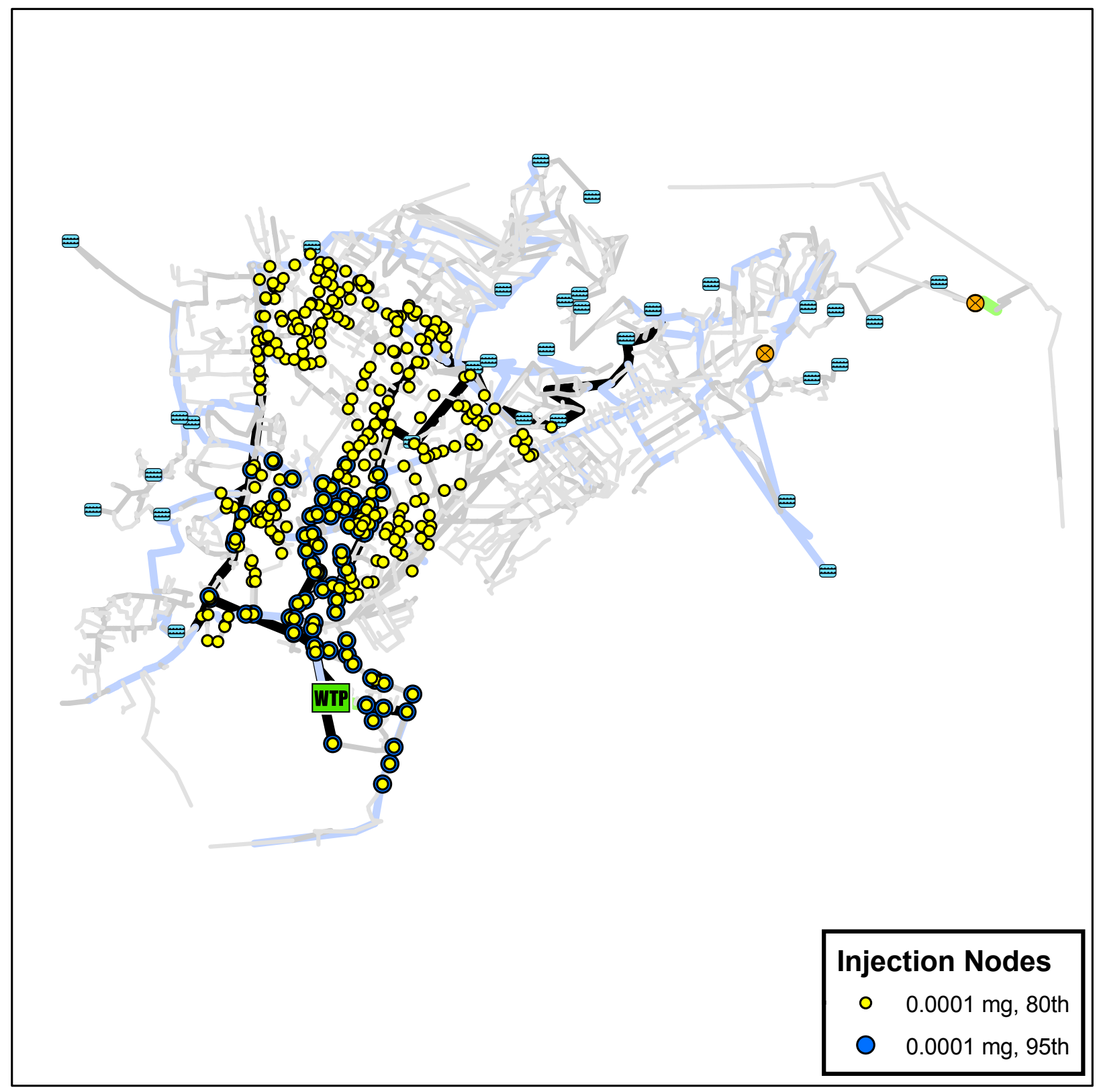

Figure 3.6. Network 4 showing locations of injection nodes associated with $80^{\text {th }}$ and $95^{\text {th }}$ percentile or higher impacts at a dose level of $0.0001 \mathrm{mg}$. 


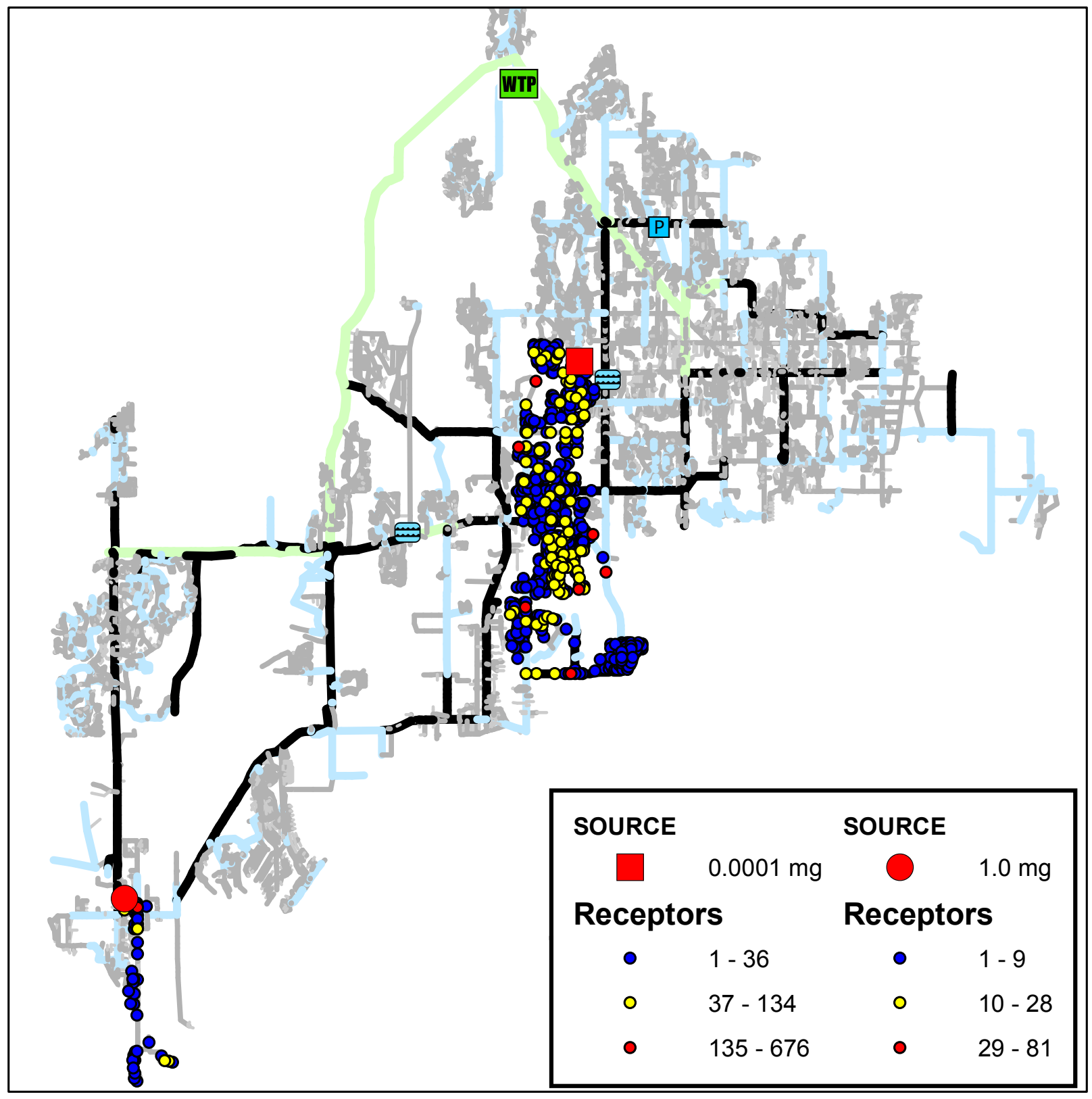

Figure 3.7. Network 6 showing receptor nodes affected following injections at the $90^{\text {th }}$ percentile nodes for dose levels of 0.0001 and $1.0 \mathrm{mg}$. The number of individuals affected at each receptor node is indicated. Additional features on the map are identified in Figure 2.3. 


\section{Section 4}

\section{Sensitivity of Impacts to Injection Duration}

Sensitivity to the various factors is considered both in terms of the statistics of the impacts across the ensemble of injection nodes and in terms of impacts associated with individual injection nodes. Figures 4.1 to 4.6 provide information on how impact statistics are affected by changes in the injection duration ( $1 \mathrm{~h}$ and $24 \mathrm{~h}$ ). Figures 4.7 to 4.13 allow an evaluation to be made of how impacts at individual injection nodes are affected. Figures 4.14 to 4.17 provide maps showing how the locations of high percentile injections nodes are influenced by changes in injection duration. Sensitivity is examined first by using Network 12 as an example and then using all networks together. This approach provides a detailed understanding of one network and an overall understanding of variability in sensitivities across all the networks.

Figures 4.1 and 4.2 provide a comparison for Network 12 of how high percentile impacts associated with 1-h and 24-h injections vary with dose level. Impacts at a given percentile decrease with increasing dose level at a rate that depends on the level of impact. The rate of decrease also varies for different networks. The figure shows that impacts associated with 24-h injections are generally larger at all dose levels and the relative difference tends to increase for lower percentile impacts. The curves in Figures 4.1 and 4.2 for the 1-h injection are the base case that is used when similar comparisons are made for the other factors.

Considering all 12 networks, Figures 4.3 and 4.4 provide plots of the relative magnitudes of the impacts for the 24-h and 1-h injections versus dose level for different percentile impacts. (For comparison, absolute values for impacts for the networks for 1-h injections are provided in Figures 3.1 to 3.3.) Generally, the impacts for the 24-h injections are larger than those for the 1-h injection because there is an increased opportunity for exposure. The ratio tends to increase with dose level, especially as the percentile of the impact decreases. However, there are considerable differences among the networks. Results are not shown if impacts for either 1-h or 24-h injections are zero.

Returning to Network 12, Figures 4.5 and 4.6 compare the distribution of impacts across all injection nodes for 1-h and 24-h injections for different dose levels using violin plots (note the change in vertical scale factor for dose levels greater than $0.1 \mathrm{mg}$ ). The distributions of impacts for the 1-h and 24-h cases are very similar for the lowest dose level $(0.0001 \mathrm{mg})$ but differ greatly for the higher dose level of $0.1 \mathrm{mg}$. Median values are similar for the two cases at the lowest dose levels and at the highest dose levels (where they are both zero), with the relative difference being largest for intermediate dose levels. The distribution for the 1-h injection is also the base case for similar comparisons for other factors.

To show how impacts associated with individual injection nodes vary as injection duration 
changes, Figures 4.7 and 4.8 compare impacts for Network 12 for the two injection durations using scatter plots. Impacts for the 1-h and the 24-h injections are compared node-by-node for all 1400 injection nodes. Impacts for the 24 -h injection are generally larger (most points lie above the red lines, which have a slope of 1 and represent equal impacts for the two injection times). The correlation between the two sets of impacts decreases as the dose level increases. Note the change in scale factors for dose levels greater than $0.1 \mathrm{mg}$.

Focusing only on higher percentile nodes, the impacts compared in the scatter plots in Figures 4.9 and 4.10 are all at or above the $80^{\text {th }}$ percentile for the 1-h injection (green points), for the 24-h injection (blue points), or both (red points). The red points identify the most critical injection locations; they are associated with impacts at the $80^{\text {th }}$ percentile and above for either 1-h or 24-h injections. The dashed lines show the $80^{\text {th }}$ percentile values. If the same injection nodes had impacts at or above the $80^{\text {th }}$ percentile for both 1-h and 24-h injection durations, then there would be 280 red points $^{1}$. If there were no overlap, there would be no red points, but there would be 280 blue points and 280 green points. Overlap decreases as the dose level increases up to a level of $1 \mathrm{mg}$. At higher dose levels, the $80^{\text {th }}$ percentile impacts are zero for both cases and, therefore, all nodes are associated with impacts above the $80^{\text {th }}$ percentile (are red). (Actual values of overlap for the various dose levels can be obtained from Figure 4.11.) Again, note the change in scale factors for dose levels greater than $0.1 \mathrm{mg}$.

Figure 4.11 provides a plot for Network 12 of the overlap of high percentile nodes for 1-h and $24-\mathrm{h}$ injections as a function of impact percentile. For example, for the $80^{\text {th }}$ percentile and a dose level of $0.1 \mathrm{mg}$, about $40 \%$ of the possible 280 nodes are $80^{\text {th }}$ percentile or higher injection nodes for both injection durations. (This quantifies the overlap that can be seen in the scatterplot in Figure 4.9 for a dose level of $0.1 \mathrm{mg}$.) The overlap of high percentile nodes for the two injection durations tends to decrease as the percentile increases and as the dose level increases. However, for the lowest dose levels $(0.0001$ and $0.001 \mathrm{mg})$ the overlap is approximately constant until the percentile is higher than $90 \%$. The overlap of high percentile nodes for high dose levels is low. In other words, for contaminants with relatively high thresholds for adverse effects, the injection locations associated with the highest impacts are quite sensitive to injection duration.

Considering all the networks, Figures 4.12 and 4.13 show the overlap of high-percentile nodes for different dose levels as a function of impact percentile. There is considerable variability by network, but, as in the case for Network 12, overlap tends to decrease as the percentile increases and as the dose level increases.

Using the map for Network 4 (see Figure 2.2), Figure 4.14 illustrates the overlap of injection nodes associated with impacts at or above the $95^{\text {th }}$ percentile for injection durations of $1 \mathrm{~h}$ and $24 \mathrm{~h}$ for a dose level of $0.0001 \mathrm{mg}$. Consistent with the results in Figure 4.12, there is high overlap of the injection nodes. Figure 4.15 provides a similar comparison for a dose level of $1.0 \mathrm{mg}$. At this dose level, little overlap of the injection nodes occurs. Figures 4.16 and 4.17 show comparisons for Network 6 for the two dose levels. Again there is high overlap of injection nodes at a dose level of $0.0001 \mathrm{mg}$ and poor overlap at $1.0 \mathrm{mg}$.

In summary, when compared on the basis of the ranking of the impact (e.g., the $90^{\text {th }}$ percentile), the impacts associated with 1-h and 24-h injections often are similar, but the degree of similarity varies by network and tends to decrease at higher dose levels and for lower impact levels (e.g., compare the $99^{\text {th }}$ and $50^{\text {th }}$ percentile impacts in Figures 4.3 and 4.4, respectively). When compared

\footnotetext{
${ }^{1}$ The network has 1400 injection nodes. Twenty per cent of these $(0.2 \times 1400=280)$ are at or above the $80^{\text {th }}$ percentile.
} 
on the basis of individual injection nodes, the impacts associated with 1-h and 24-h injections can vary substantially (Figures 4.7 to 4.10 ). The overlap of the high percentile nodes for the two injection durations can be low for higher dose levels with higher impact.

The series of figures used to examine the sensitivity of impacts to injection duration are repeated in the examination of the other factors. 


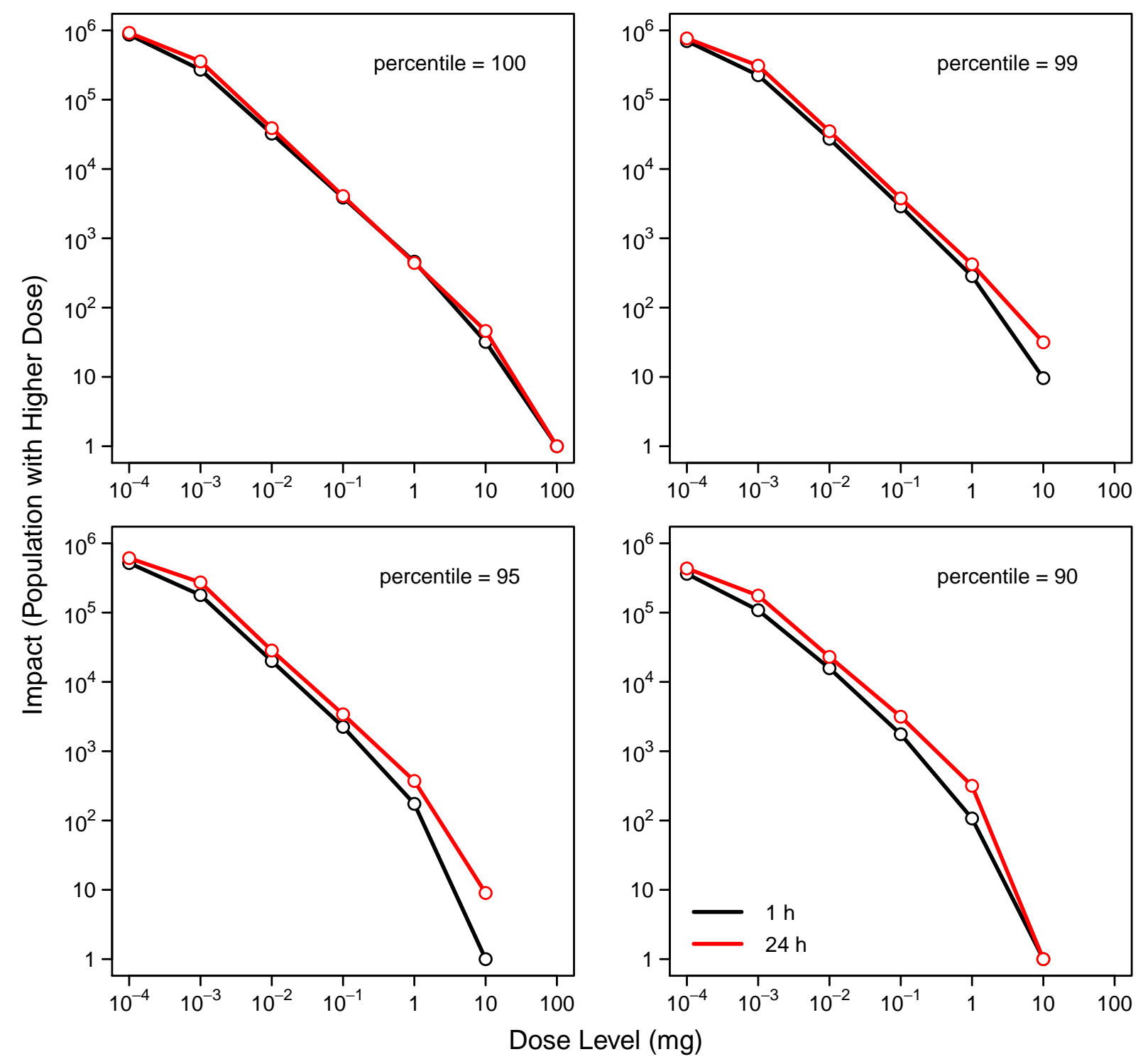

Figure 4.1. Impacts associated with 1-h and 24-h injections for Network 12 as a function of dose level, for the $90^{\text {th }}, 95^{\text {th }}, 99^{\text {th }}$, and $100^{\text {th }}$ percentile injection nodes. 


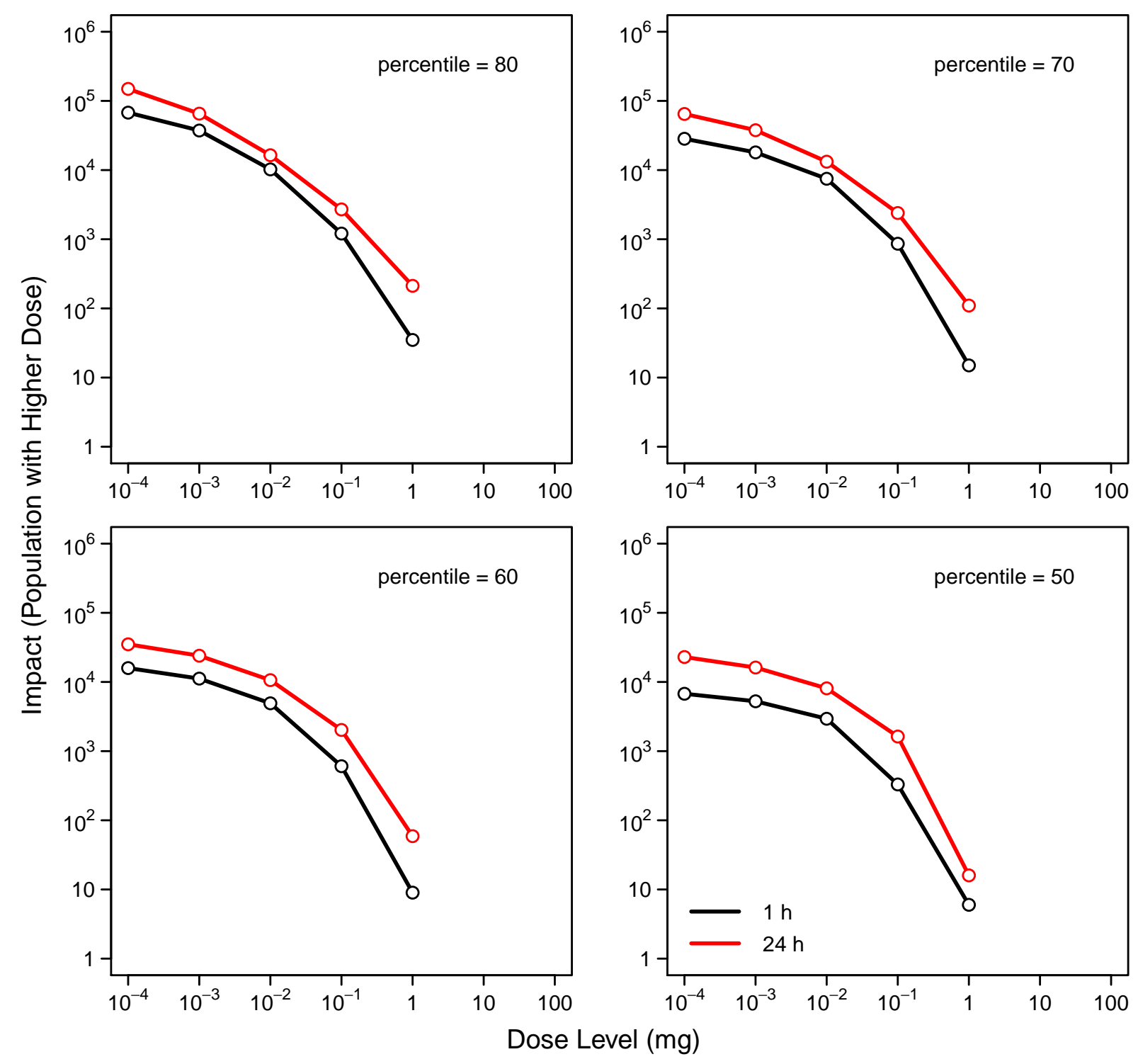

Figure 4.2. Impacts associated with 1-h and 24-h injections for Network 12 as a function of dose level, for the $50^{\text {th }}, 60^{\text {th }}, 70^{\text {th }}$, and $80^{\text {th }}$ percentile injection nodes. 


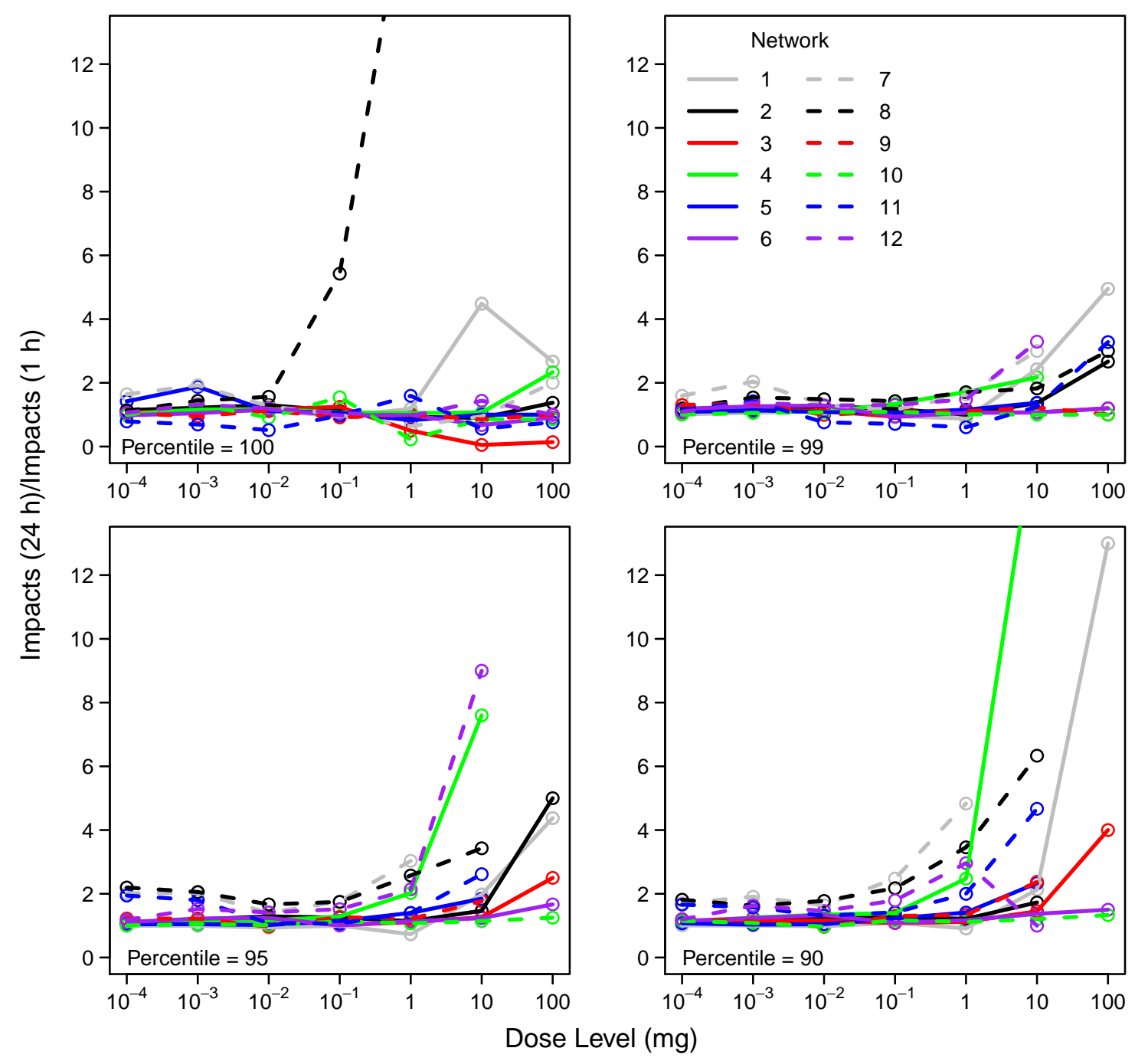

Figure 4.3. Impact for a $24-\mathrm{h}$ injection relative to the impact for a 1-h injection, as a function of dose level, for the $90^{\text {th }}, 95^{\text {th }}, 99^{\text {th }}$, and $100^{\text {th }}$ percentile injection nodes. 


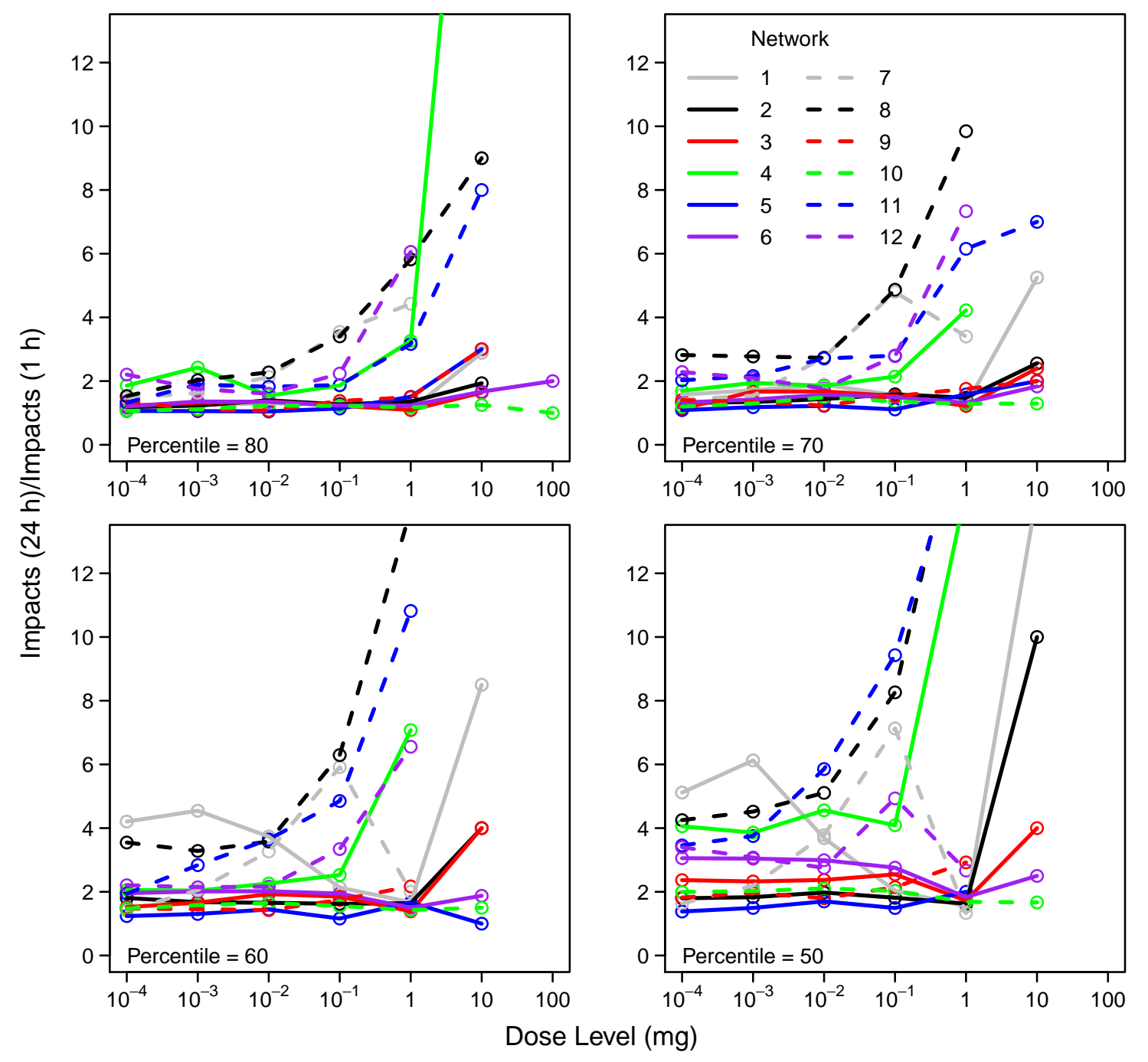

Figure 4.4. Impact for a 24-h injection relative to the impact for a 1-h injection, as a function of dose level, for the $50^{\text {th }}, 60^{\text {th }}, 70^{\text {th }}$, and $80^{\text {th }}$ percentile injection nodes. 


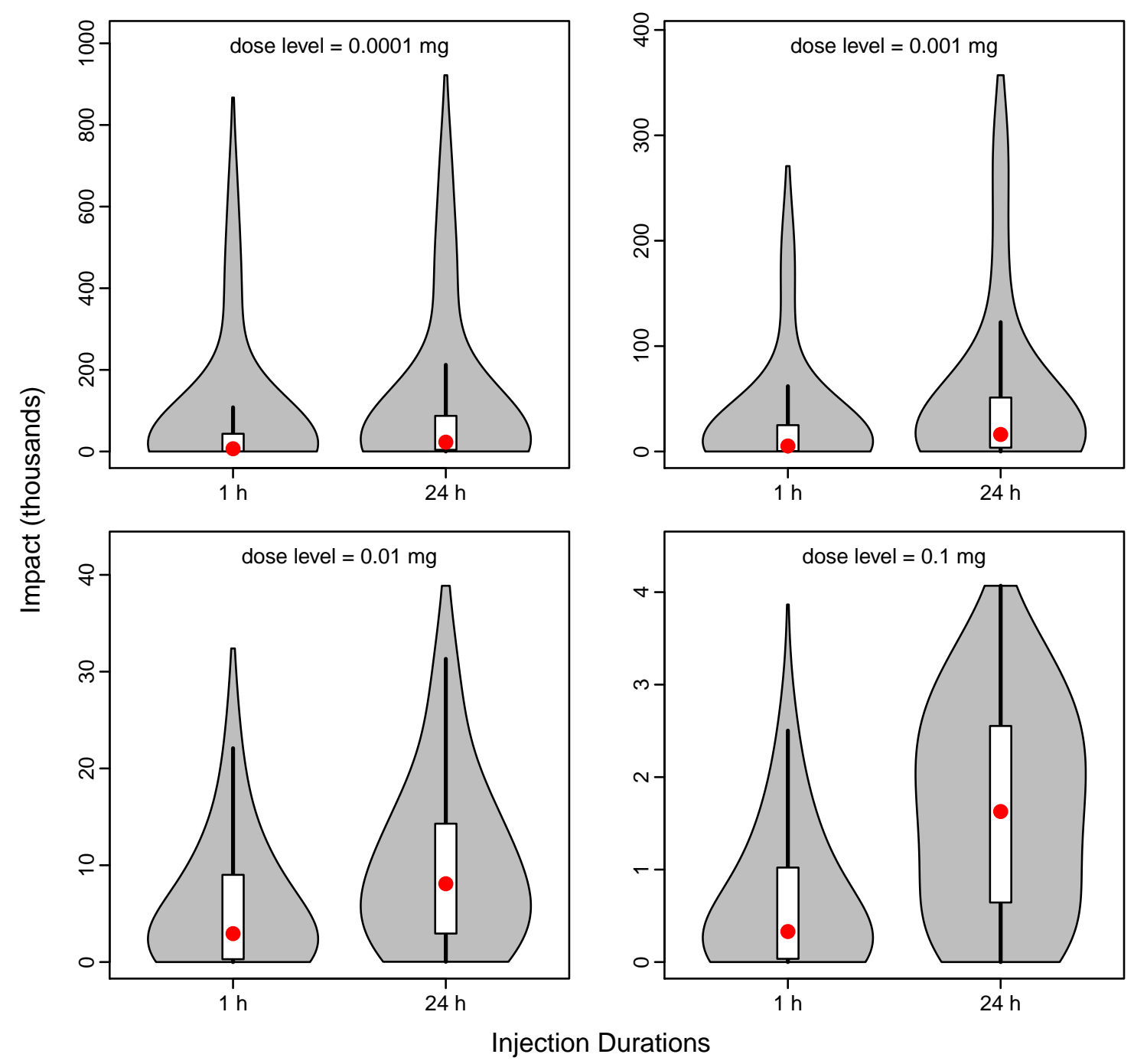

Figure 4.5. Distributions of impacts for Network 12 across all injection nodes for 1-h and 24-h injections for dose levels of $0.0001,0.001,0.01$, and $0.1 \mathrm{mg}$. The red dot represents the median value. 


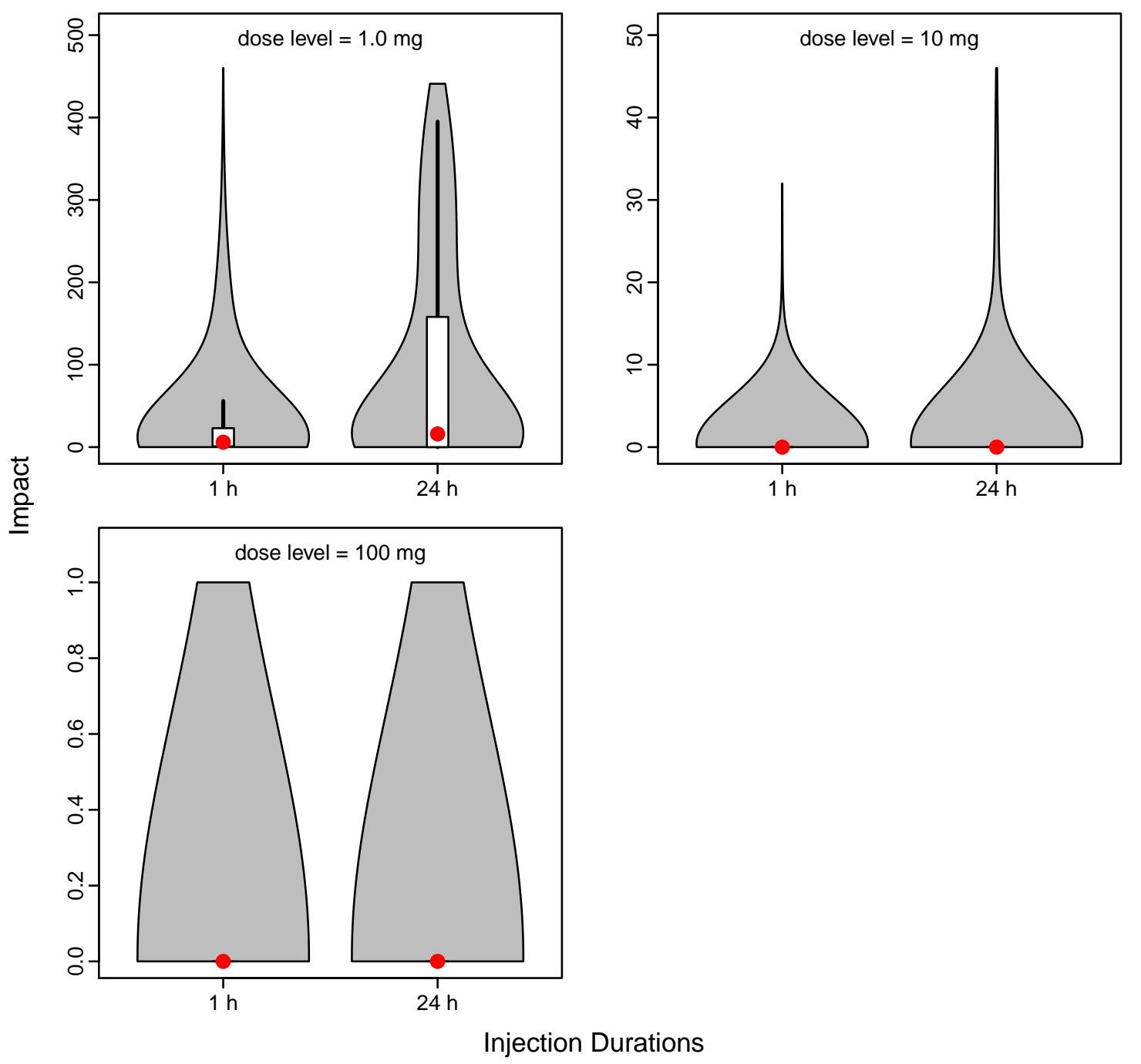

Figure 4.6. Distributions of impacts for Network 12 across all injection nodes for 1-h and 24-h injections for dose levels of 1,10 , and $100 \mathrm{mg}$. 


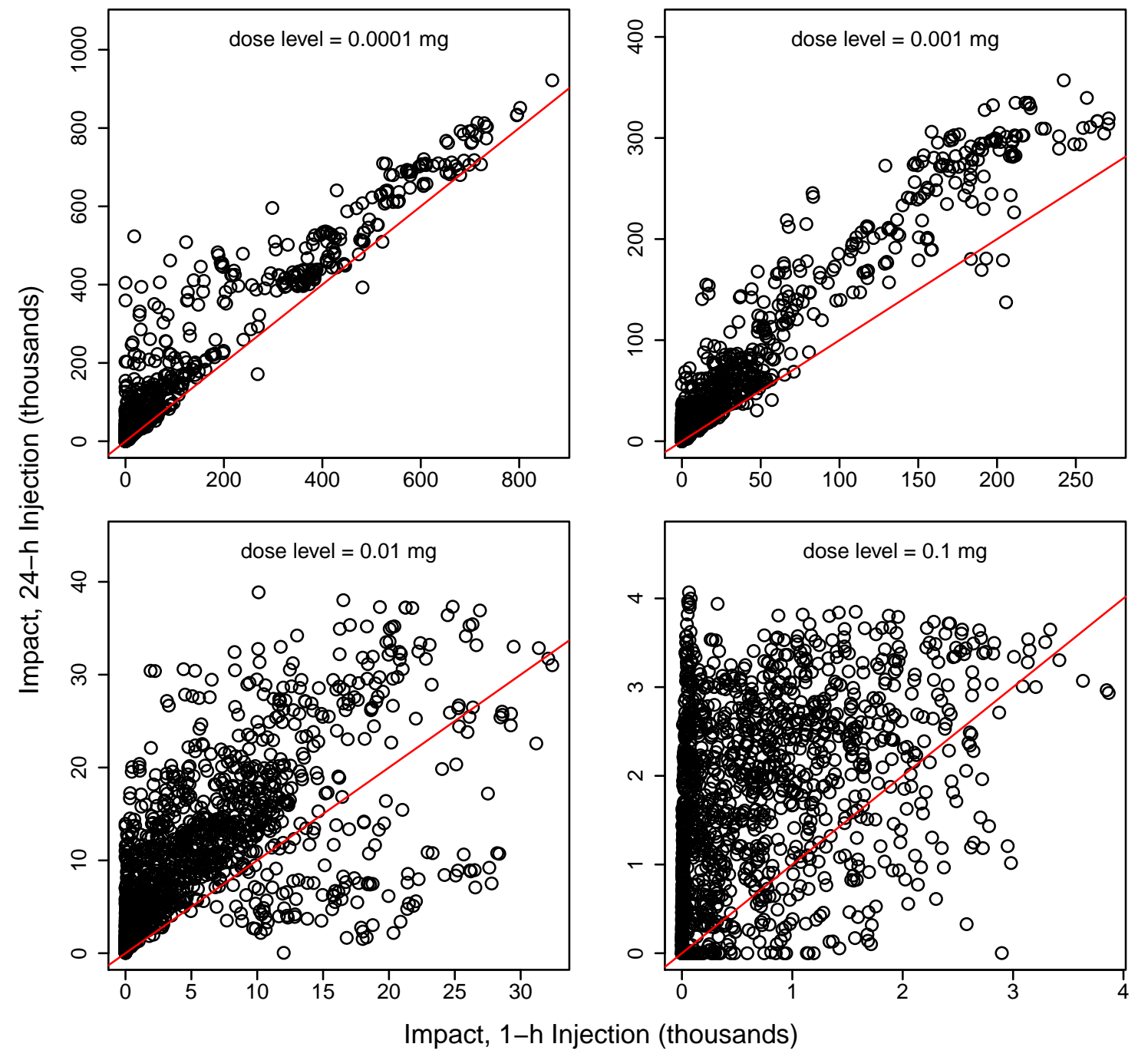

Figure 4.7. Impacts for Network 12 for 1-h and 24-h injections for dose levels of 0.0001, $0.001,0.01$, and $0.1 \mathrm{mg}$. The red lines have a slope of 1. Each point corresponds to one injection node. 


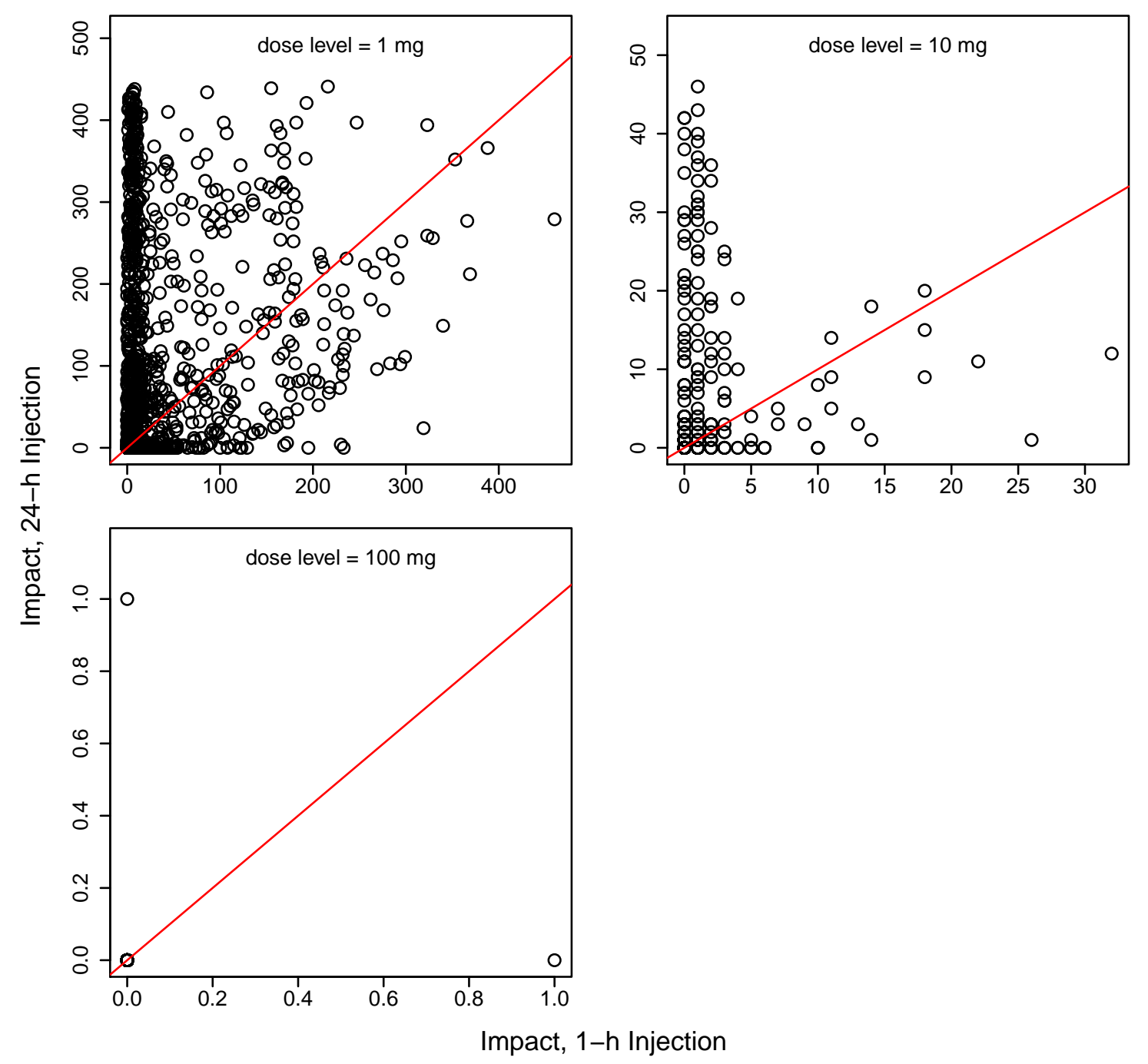

Figure 4.8. Impacts for Network 12 for 1-h and 24-h injections for dose levels of 1 , 10, and $100 \mathrm{mg}$. The red lines have a slope of 1 . Each point corresponds to one injection node. 


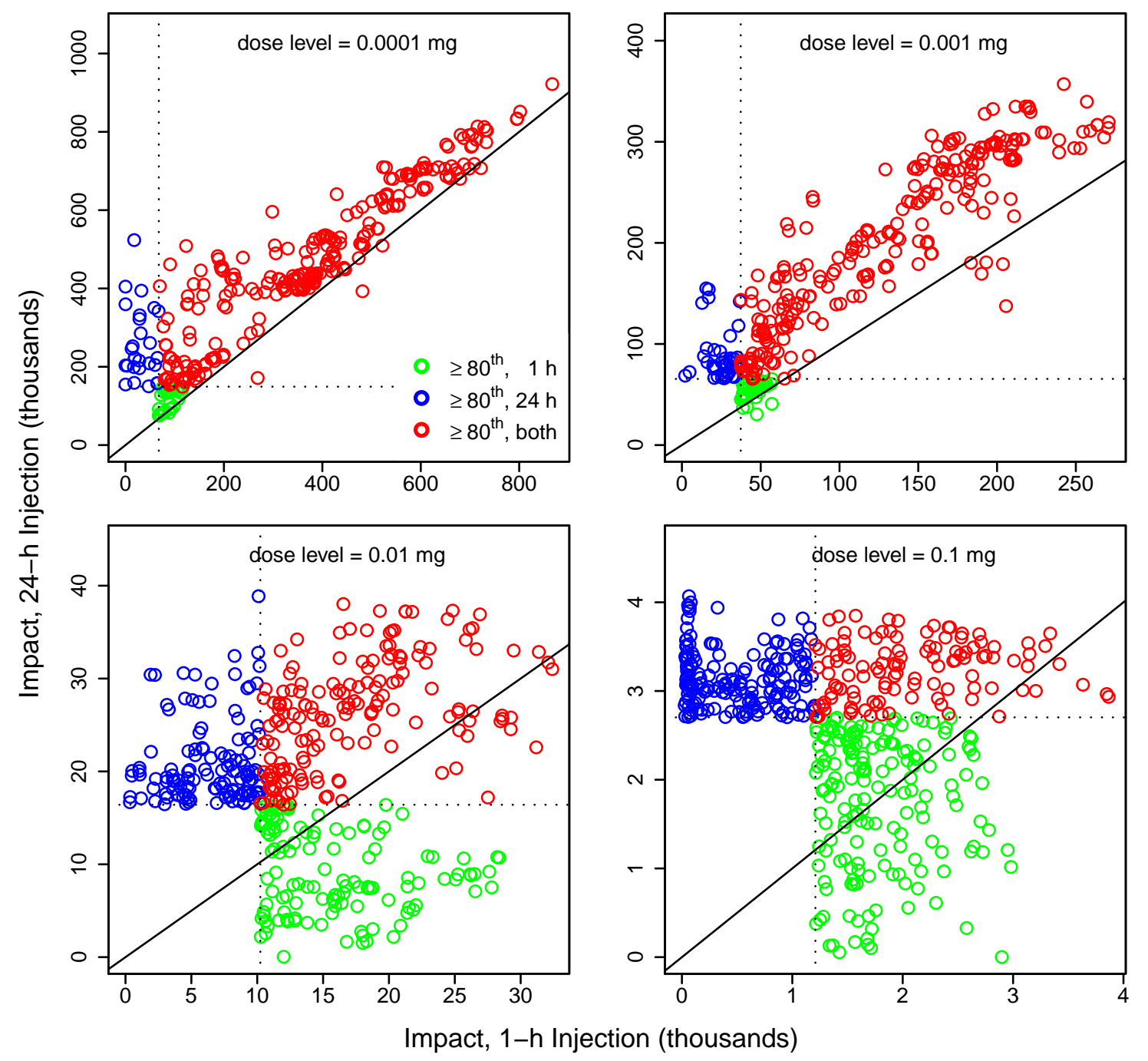

Figure 4.9. Impacts for Network 12 that are at or above the $80^{\text {th }}$ percentile level for 1 -h and/or 24-h injections for dose levels of $0.0001,0.001,0.01$, and $0.1 \mathrm{mg}$. The dotted lines indicate $80^{\text {th }}$ percentile impacts. The black lines have a slope of 1 . Each point corresponds to one injection node. 


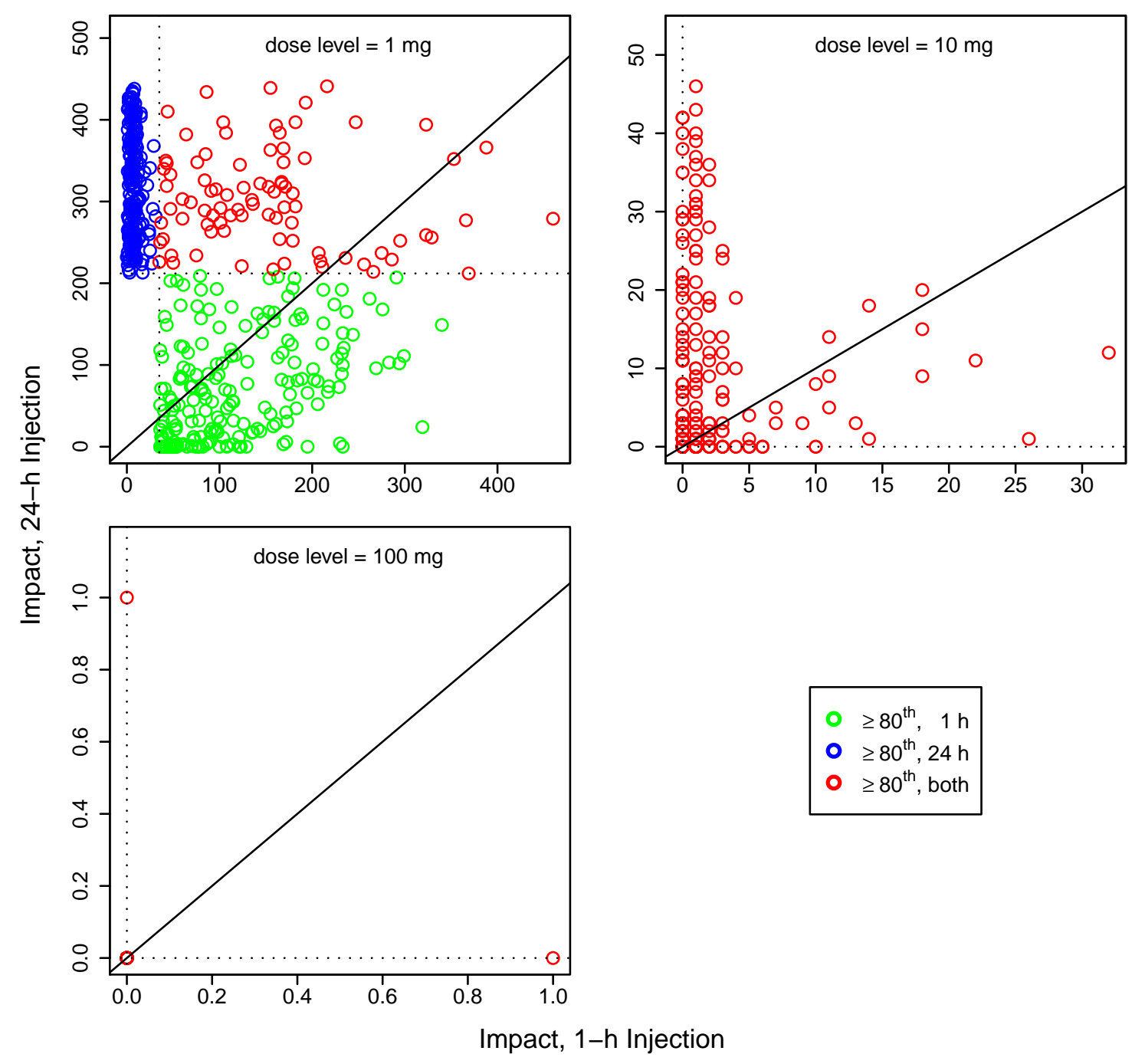

Figure 4.10. Impacts for Network 12 that are at or above the $80^{\text {th }}$ percentile level for 1 -h and/or 24-h injections for dose levels of 1,10 , and $100 \mathrm{mg}$. The dotted lines indicate $80^{\text {th }}$ percentile impacts. The black lines have a slope of 1 . Each point corresponds to one injection node. 


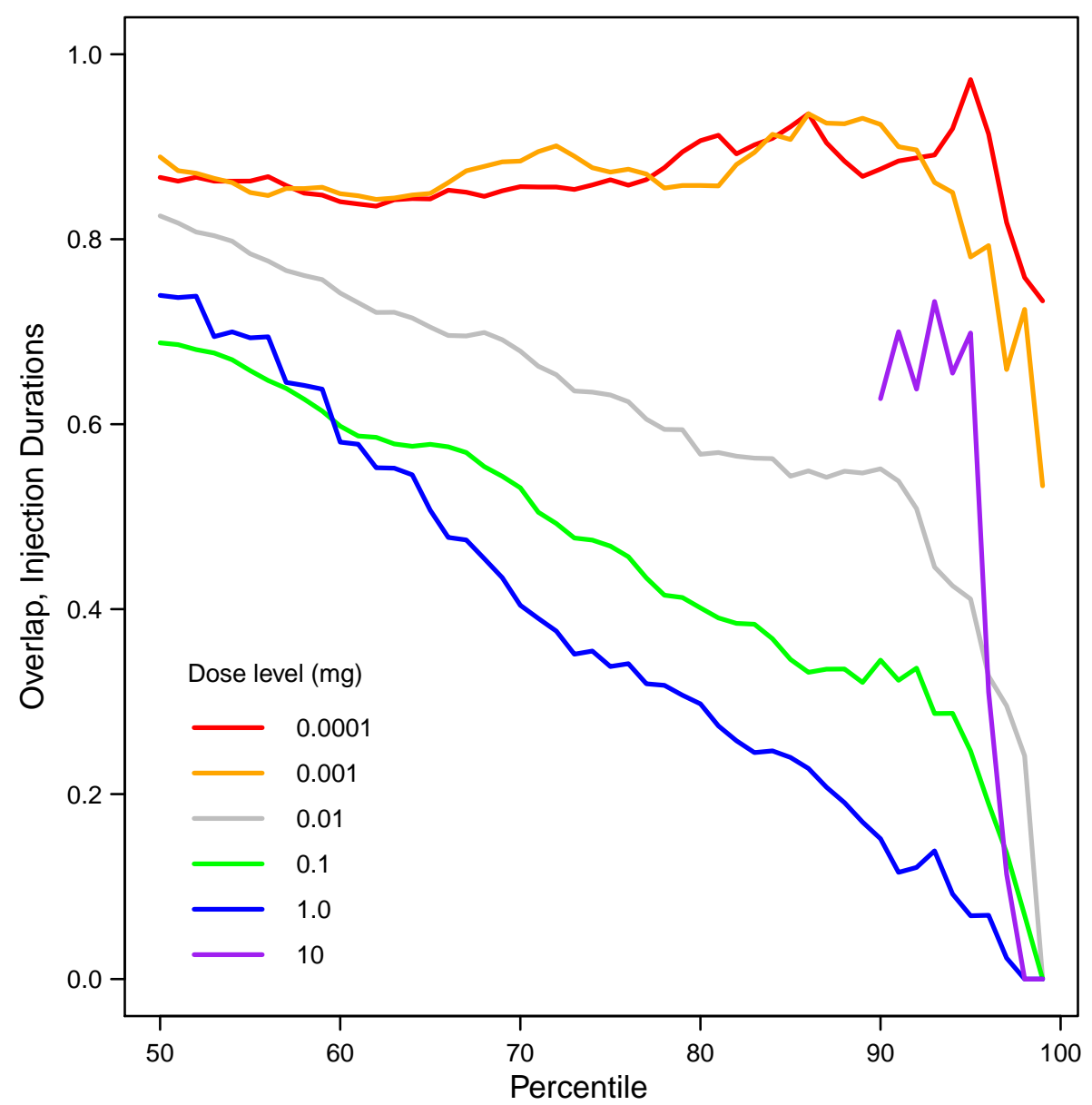

Figure 4.11. Overlap of high percentile injection nodes for Network 12 for 1-h and 24-h injections for different dose levels. 

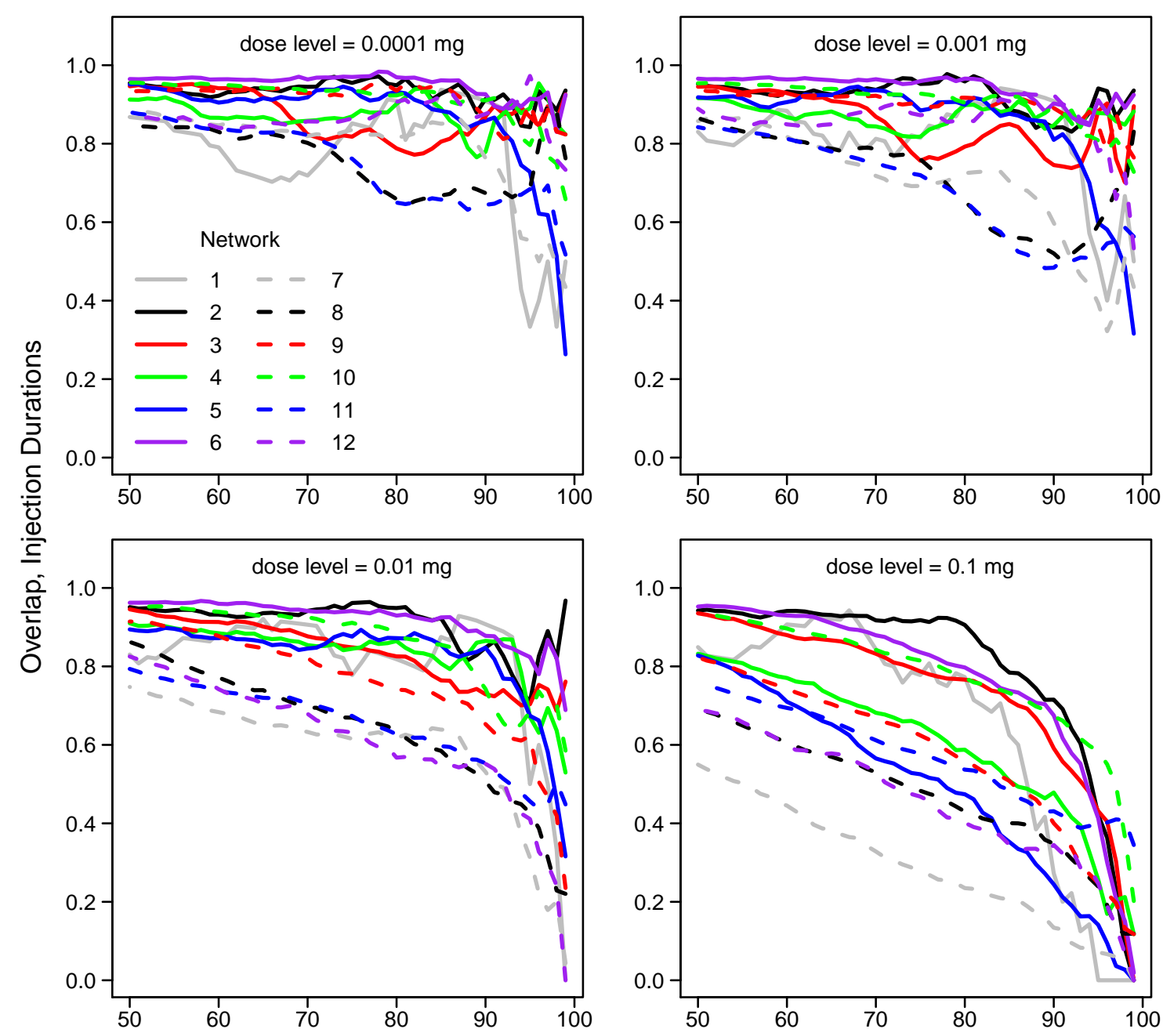

Percentile

Figure 4.12. Overlap of high percentile injection nodes for the networks for 1-h and 24-h injections for dose levels of $0.0001,0.001,0.01$, and $0.1 \mathrm{mg}$. 


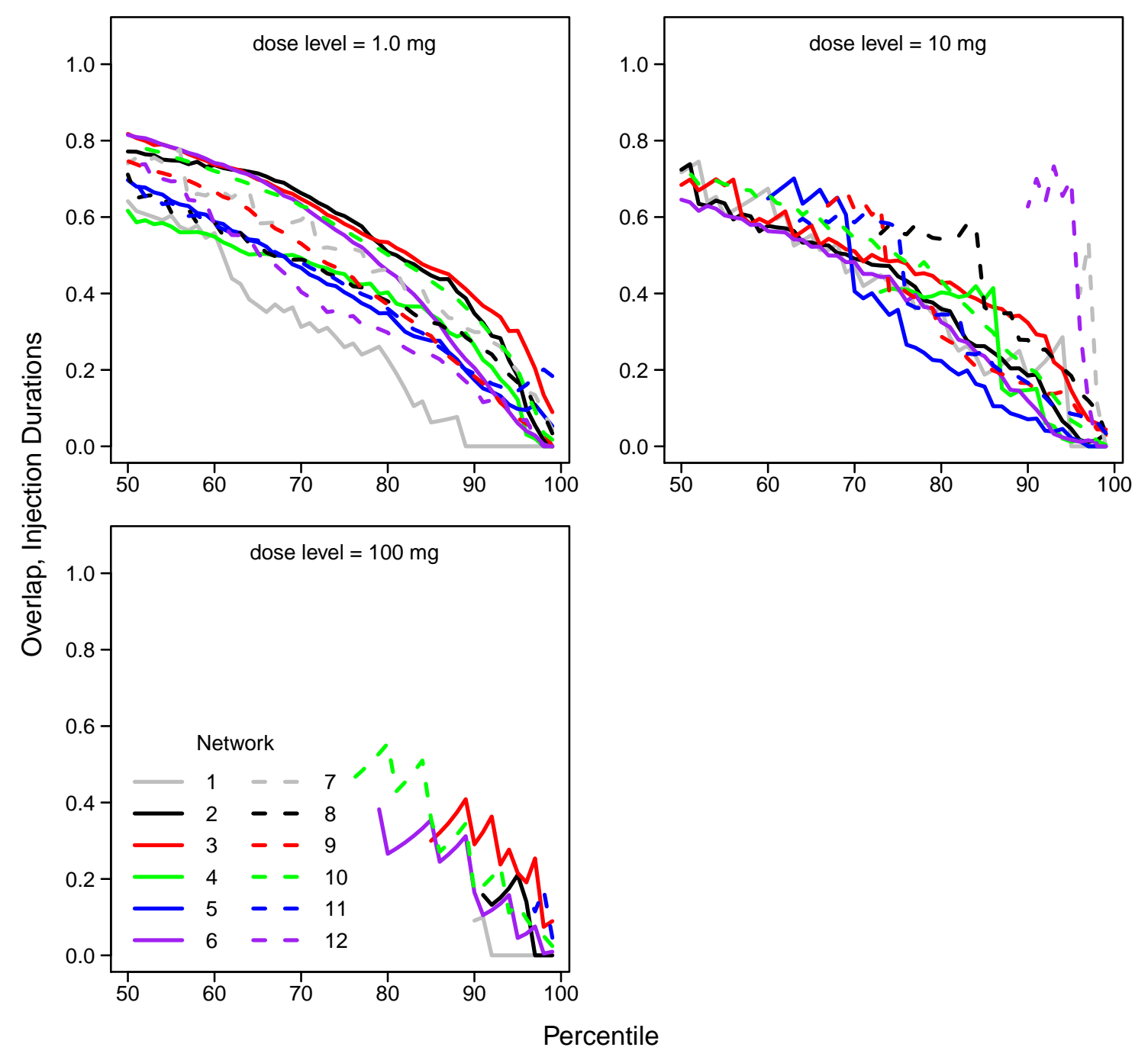

$\mathrm{S}$

Figure 4.13. Overlap of high percentile injection nodes for the networks for 1-h and 24-h injections for dose levels of 1,10 , and $100 \mathrm{mg}$. 


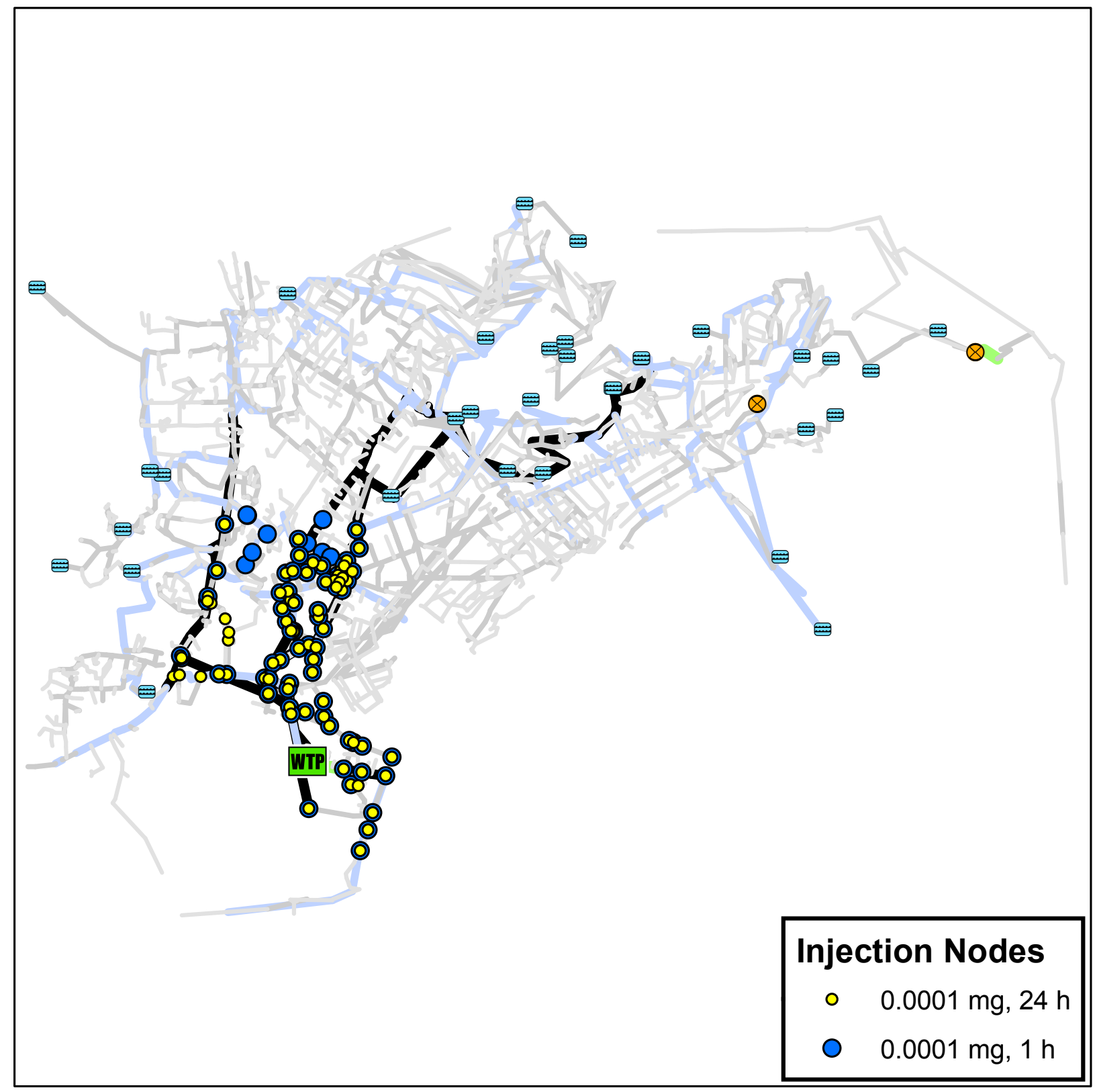

Figure 4.14. Network 4 showing locations of injection nodes associated with $95^{\text {th }}$ percentile or higher impacts at a dose level of $0.0001 \mathrm{mg}$ for injection durations of $1 \mathrm{~h}$ and $24 \mathrm{~h}$. 


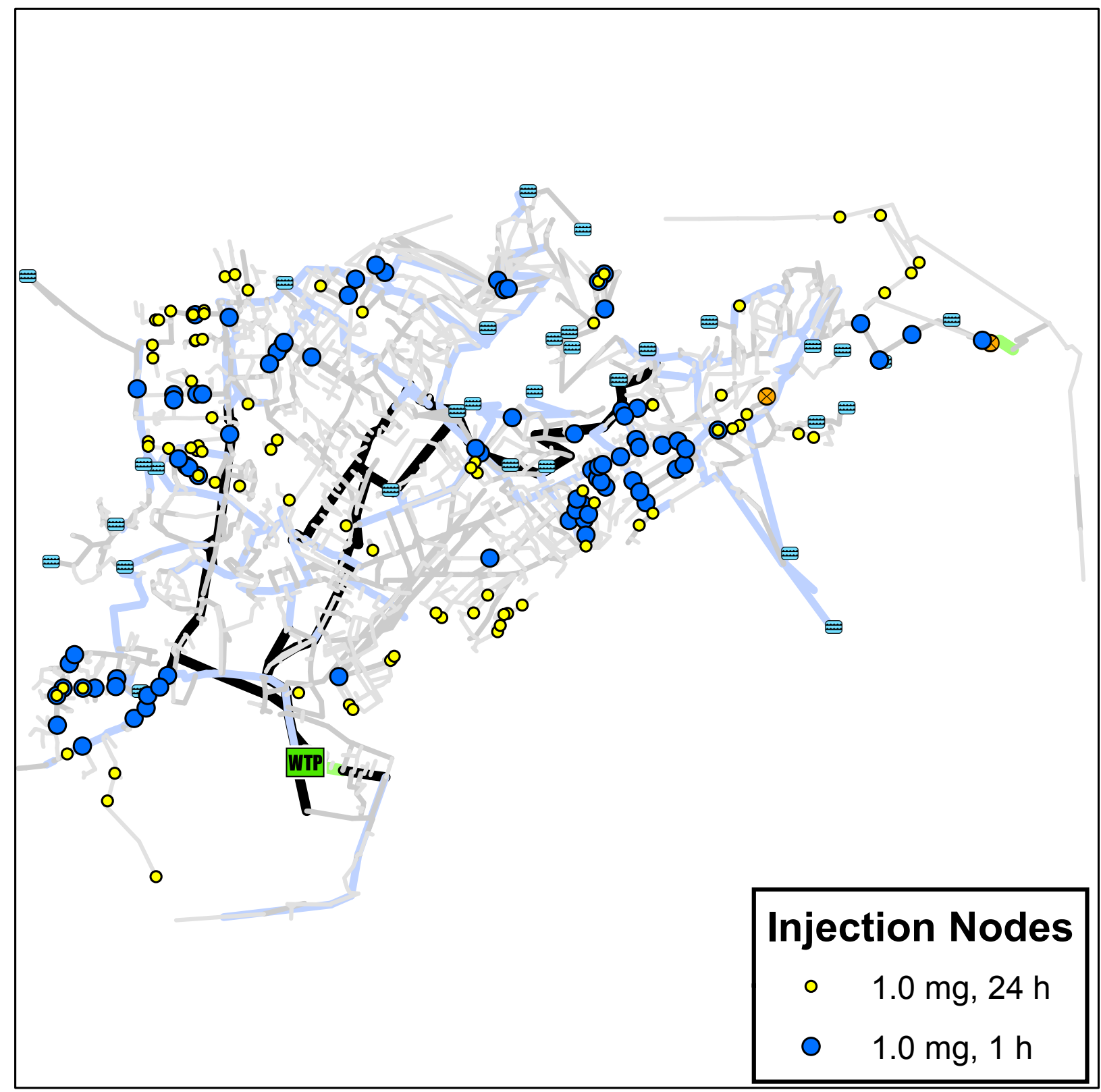

Figure 4.15. Network 4 showing locations of injection nodes associated with $95^{\text {th }}$ percentile or higher impacts at a dose level of $1.0 \mathrm{mg}$ for injection durations of $1 \mathrm{~h}$ and $24 \mathrm{~h}$. 


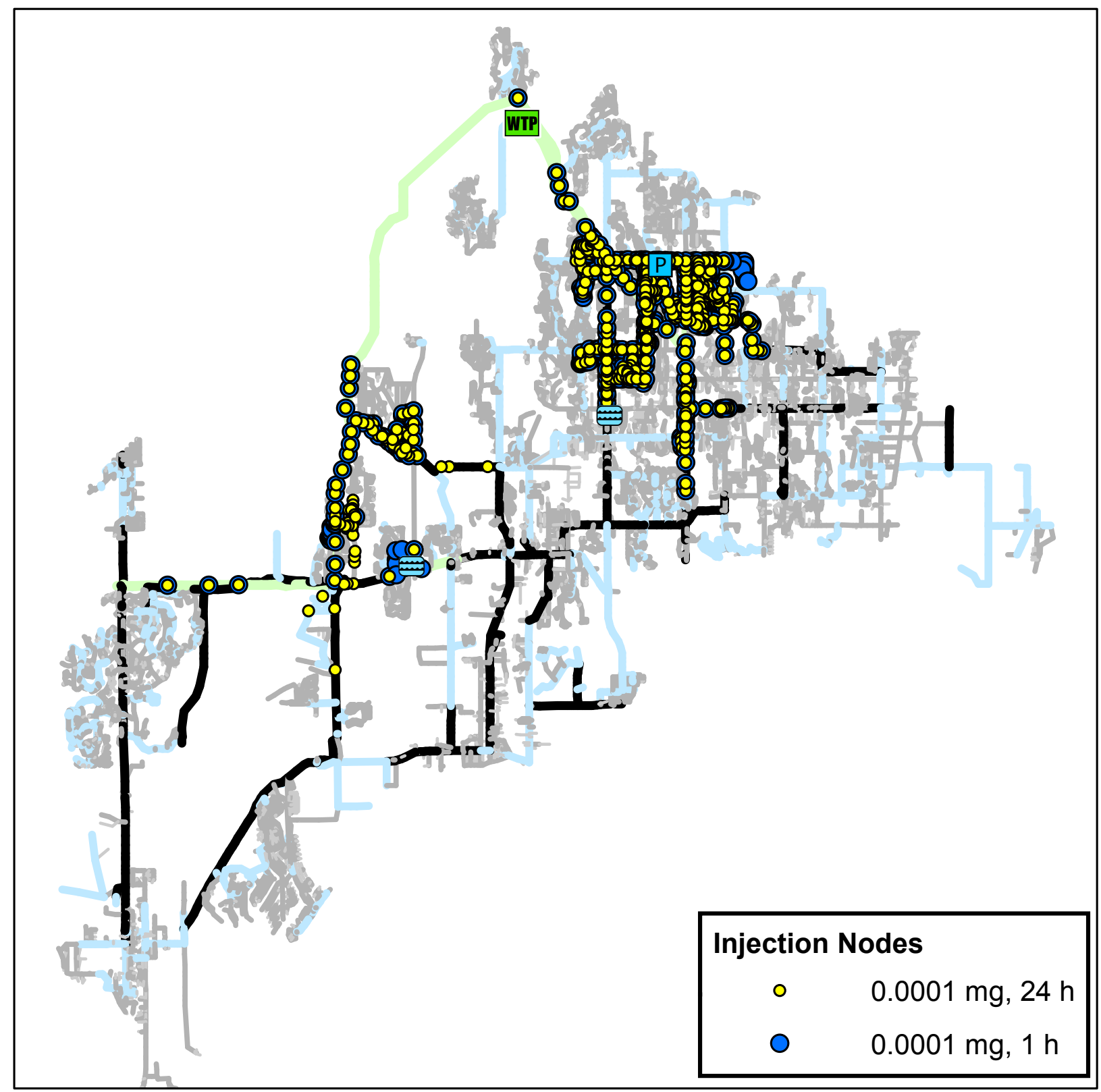

Figure 4.16. Network 6 showing locations of injection nodes associated with $95^{\text {th }}$ percentile or higher impacts at a dose level of $0.0001 \mathrm{mg}$ for injection durations of $1 \mathrm{~h}$ and $24 \mathrm{~h}$. 


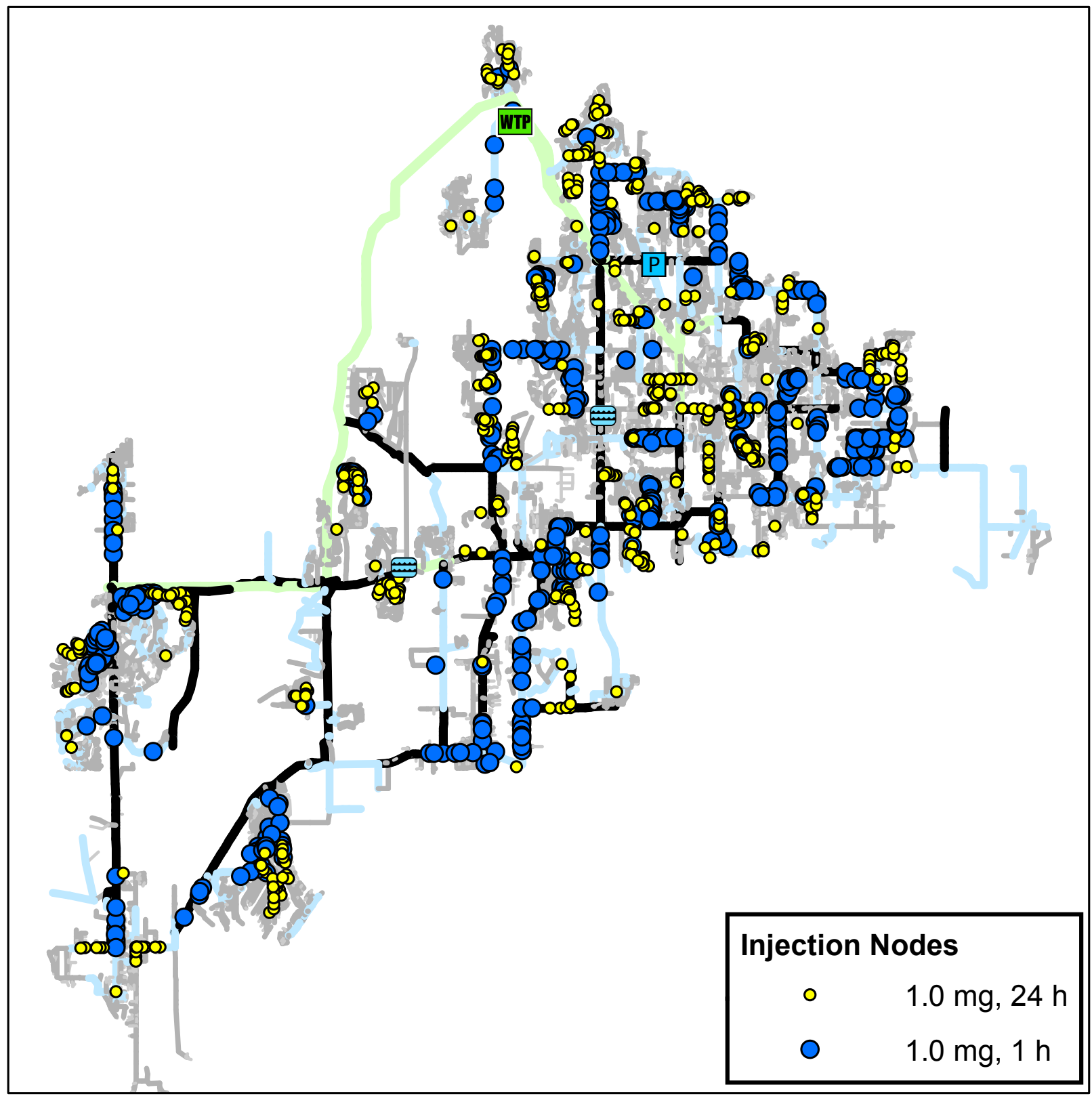

Figure 4.17. Network 6 showing locations of injection nodes associated with $95^{\text {th }}$ percentile or higher impacts at a dose level of $1.0 \mathrm{mg}$ for injection durations of $1 \mathrm{~h}$ and $24 \mathrm{~h}$. 


\section{Section 5}

\section{Sensitivity of Impacts to Injection Time}

This section addresses the sensitivity of impacts to changes in injection time. The presentation is generally similar to that used for injection duration in the previous section.

We considered four injection times, 0:00, 6:00, 12:00, and 18:00. Figures 5.1 and 5.2 show how ranked impacts vary for Network 12 for the different injection times as a function of dose level. Generally, the impacts associated with injections at 0:00 are the smallest, with the injection time associated with the largest impact varying somewhat by percentile. At low dose levels, the impacts vary little with injection time, while at higher dose levels there can be considerable difference in impacts for different injection times.

Considering all the networks, Figures 5.3 and 5.4 provide ratios of impacts for the injection time with the largest impact to impacts for the injection time with the smallest impacts, as a function of dose level. (For comparison, absolute values for impacts for the networks for injections at 0:00 are provided in Figures 3.1 to 3.3.) Results are provided for different percentile impacts. As is the case with injection duration, the ratio tends to increase with dose level, particularly as the percentile of the impact decreases. There is considerable variability among the networks and the variability increases with dose level.

Again, considering all the networks, impacts associated with the various injection times are shown in Figures 5.5 through 5.9 relative to the impact associated with an injection at 0:00. Relative impacts are provided for dose levels from $0.0001 \mathrm{mg}$ to $1.0 \mathrm{mg}$ for injection nodes with percentiles of $100 \%, 95 \%, 75 \%$, and $50 \%$. The diurnal pattern in relative impacts varies with network, dose level, and percentile. At the higher dose levels, the larger networks tend to have a larger range in impacts for different injection times than do the smaller networks. No figures similar to 5.5 through 5.9 are provided for the analysis of the sensitivity of impacts to injection duration, population model, or ingestion model because in those cases only two values of the factor were considered. However, similar figures are provided for the analysis involving injection mass.

The distributions of impacts across injection nodes are compared in Figures 5.10 and 5.11 for different injection times for Network 12. There can be considerable differences in the distributions for the different injection times, depending on dose level. For example, compare the distributions for a dose level of $0.1 \mathrm{mg}$.

The scatter plots in Figures 5.12 through 5.17 compare impacts by injection node for injection times of 6:00, 12:00, and 18:00 with the impacts for the same nodes for an injection time of 0:00, for Network 12. Impacts associated with injections at 0:00 are generally smaller than those associated with the other injection times (most points lie above the red line). (However, as Figures 
5.5 through 5.9 show, this behavior is not always true for all networks.) The degree of scatter increases with dose level, up to a dose level of about $1 \mathrm{mg}$.

The scatter plots in Figures 5.18 through 5.23 include only those injection nodes for Network 12 for which impacts are at or above the $80^{\text {th }}$ percentile for at least one of the injection times. The red points in the plots are associated with injection nodes whose impacts are at or above the $80^{\text {th }}$ percentile for both injection times used in a plot. The overlap of nodes that are at or above the $80^{\text {th }}$ or other high percentile for both cases decreases considerably as the dose level increases, at least up to a dose level of about $0.1 \mathrm{mg}$, as is shown explicitly in Figures 5.24, 5.25, and 5.26. Even for the lower dose levels, the degree of overlap can decrease considerably for higher percentile impacts.

Considering all the networks, the overlap of the high percentile injection nodes is shown in the plots in Figures 5.27 through 5.32 for injection times of 6:00, 12:00, and 18:00, compared with an injection time of 0:00. The plots show how overlap varies with dose level for different percentile impacts. The overlap varies considerably among the networks, but tends to decrease with the rank of the impact and the dose level.

Figures 5.33 through 5.38 provide maps of Network 4 comparing the locations of $95^{\text {th }}$ percentile and higher injection nodes for different injection times (0:00, 6:00, 12:00, and 18:00) for dose levels of $0.0001 \mathrm{mg}$ (Figures 5.33 to 5.35 ) and $1.0 \mathrm{mg}$ (Figures 5.36 to 5.38). Figures 5.39 through 5.44 provide similar maps for Network 6. For both networks, high overlap of injection nodes occurs for the different injection times for a dose level of $0.0001 \mathrm{mg}$. For a dose level of $1.0 \mathrm{mg}$, the overlap of injection nodes for both networks is much lower. These results are consistent with the overlap plots for the networks given in Figures 5.27 through 5.32.

Overall, the sensitivity of impacts to injection time is similar to the sensitivity to injection duration. However, the variability in sensitivity of ranked impacts for the different networks is generally higher for injection times than for injection duration (cf. Figures 4.3 and 5.3). 


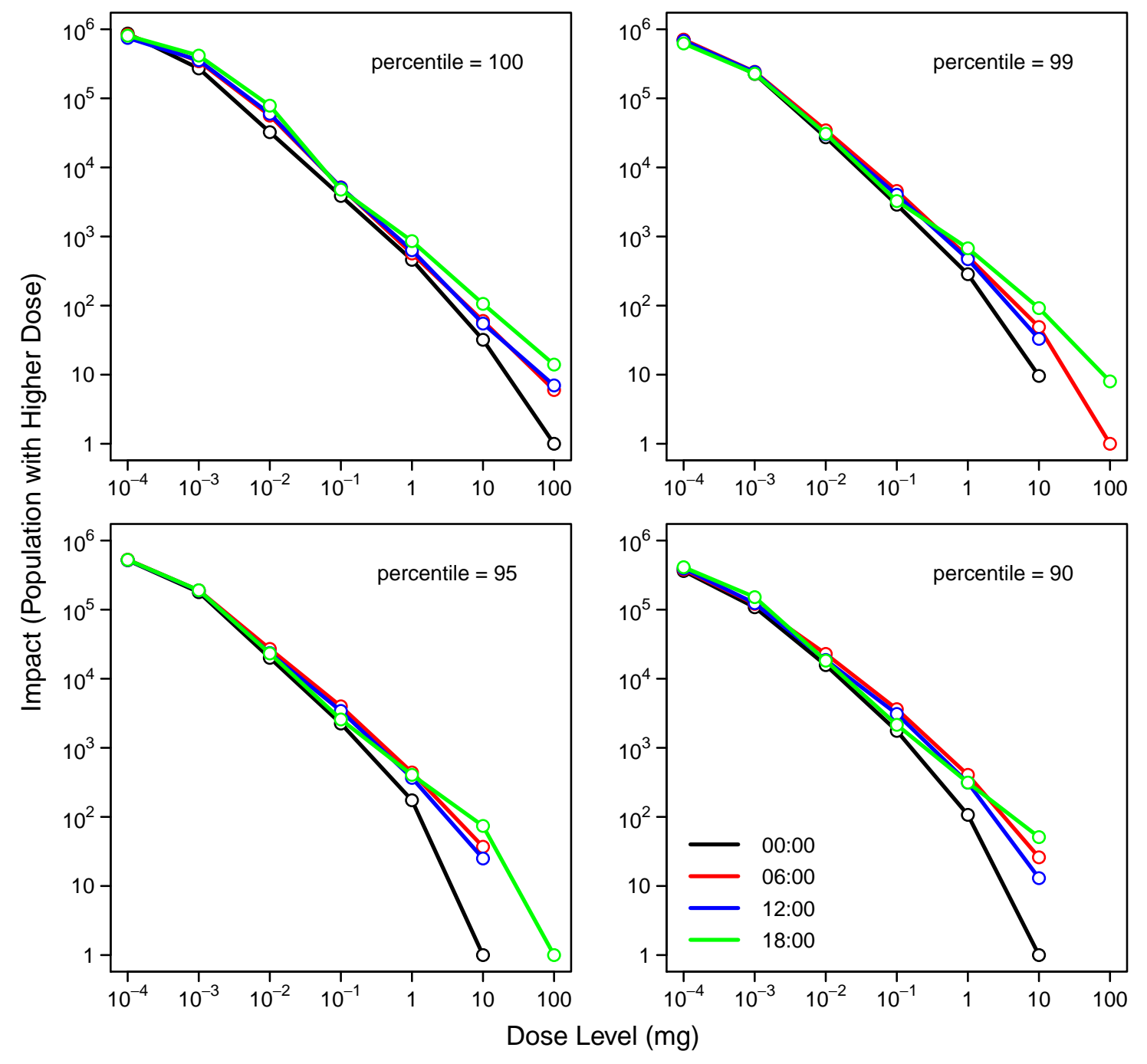

Figure 5.1. Impacts associated with different injection times for Network 12 as a function of dose level, for the $90^{\text {th }}, 9^{\text {th }}, 99^{\text {th }}$, and $100^{\text {th }}$ percentile injection nodes. 


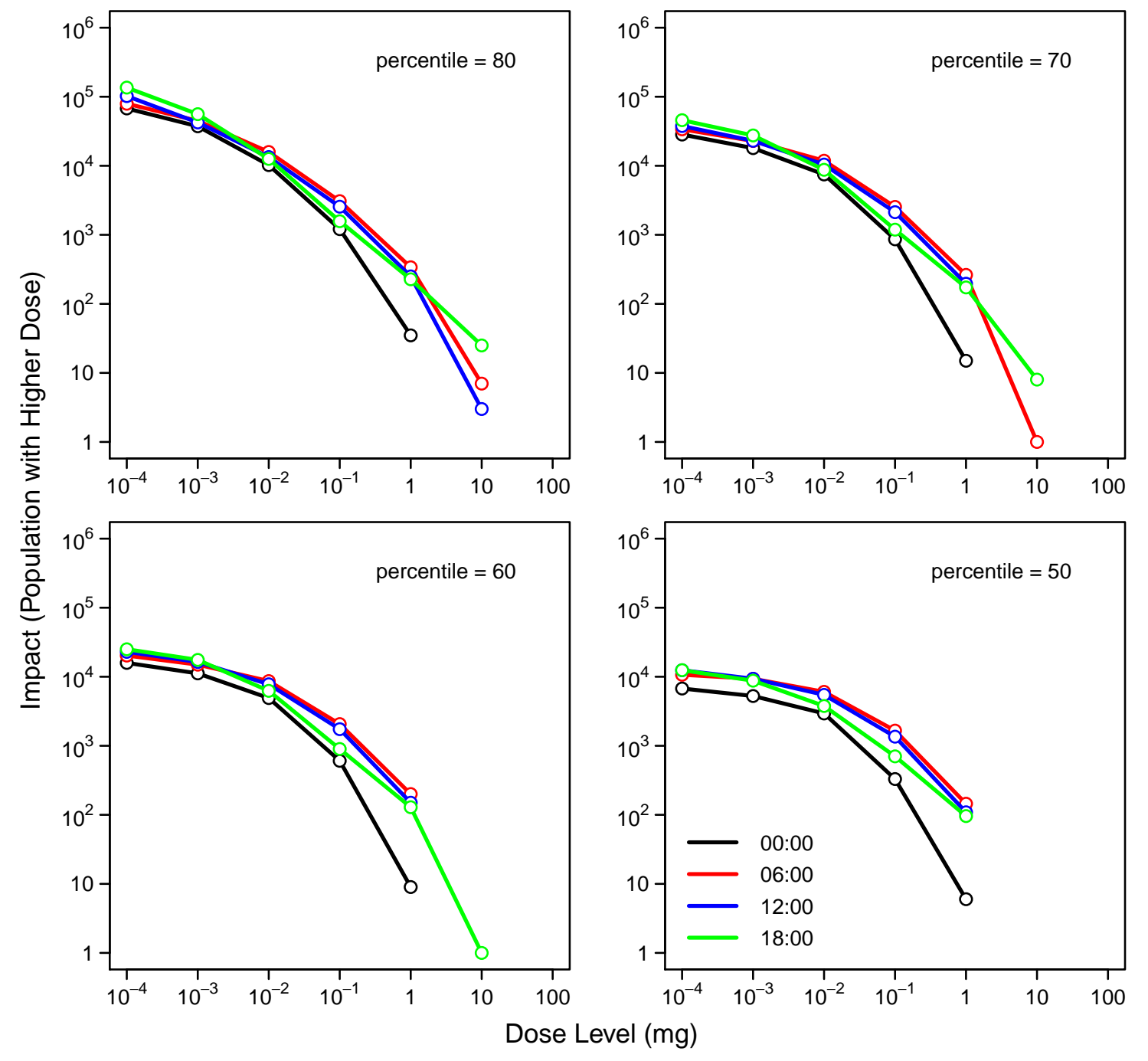

Figure 5.2. Impacts associated with different injection times for Network 12 as a function of dose level, for the $50^{\text {th }}, \mathbf{6 0}^{\text {th }}, 7^{\text {th }}$, and $80^{\text {th }}$ percentile injection nodes. 


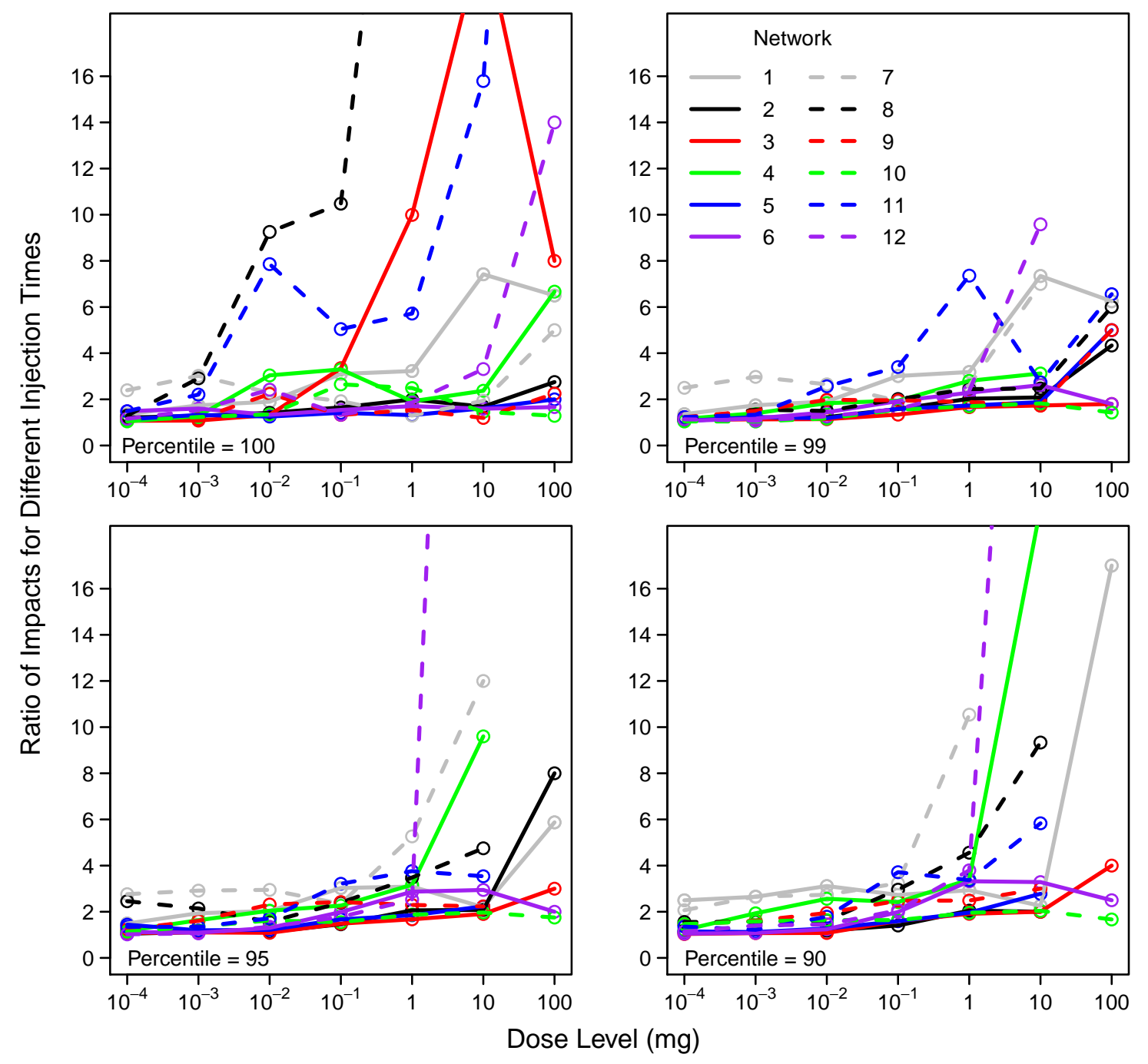

Figure 5.3. Maximum ratio of impacts for the networks for four different injection times as a function of dose level, for the $90^{\text {th }}, 95^{\text {th }}, 99^{\text {th }}$, and $100^{\text {th }}$ percentile injection nodes. 


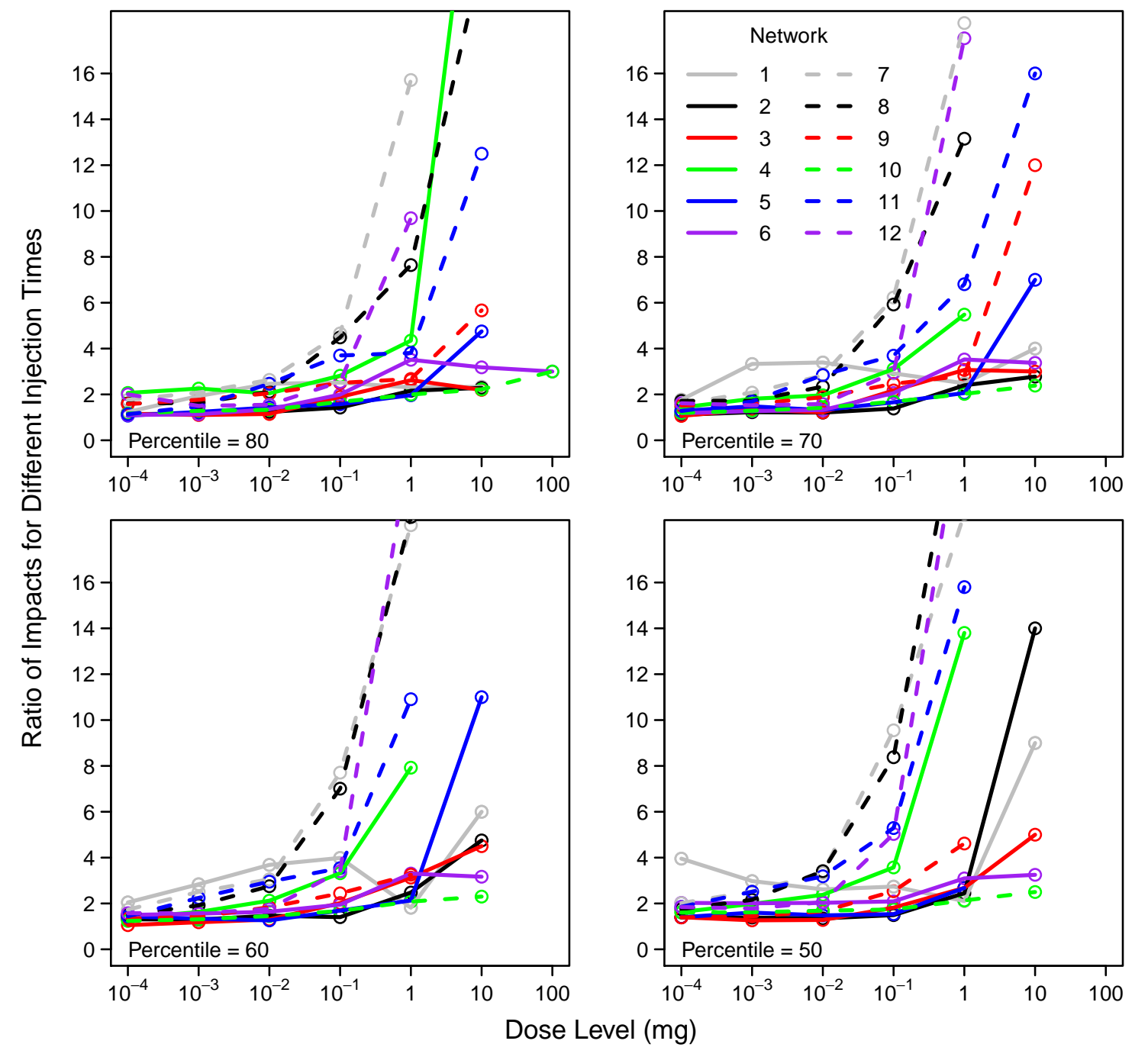

Figure 5.4. Maximum ratio of impacts for the networks for four different injection times as a function of dose level, for the $50^{\text {th }}, 60^{\text {th }}, 70^{\text {th }}$, and $80^{\text {th }}$ percentile injection nodes. 
Dose Level $=0.0001 \mathrm{mg}$

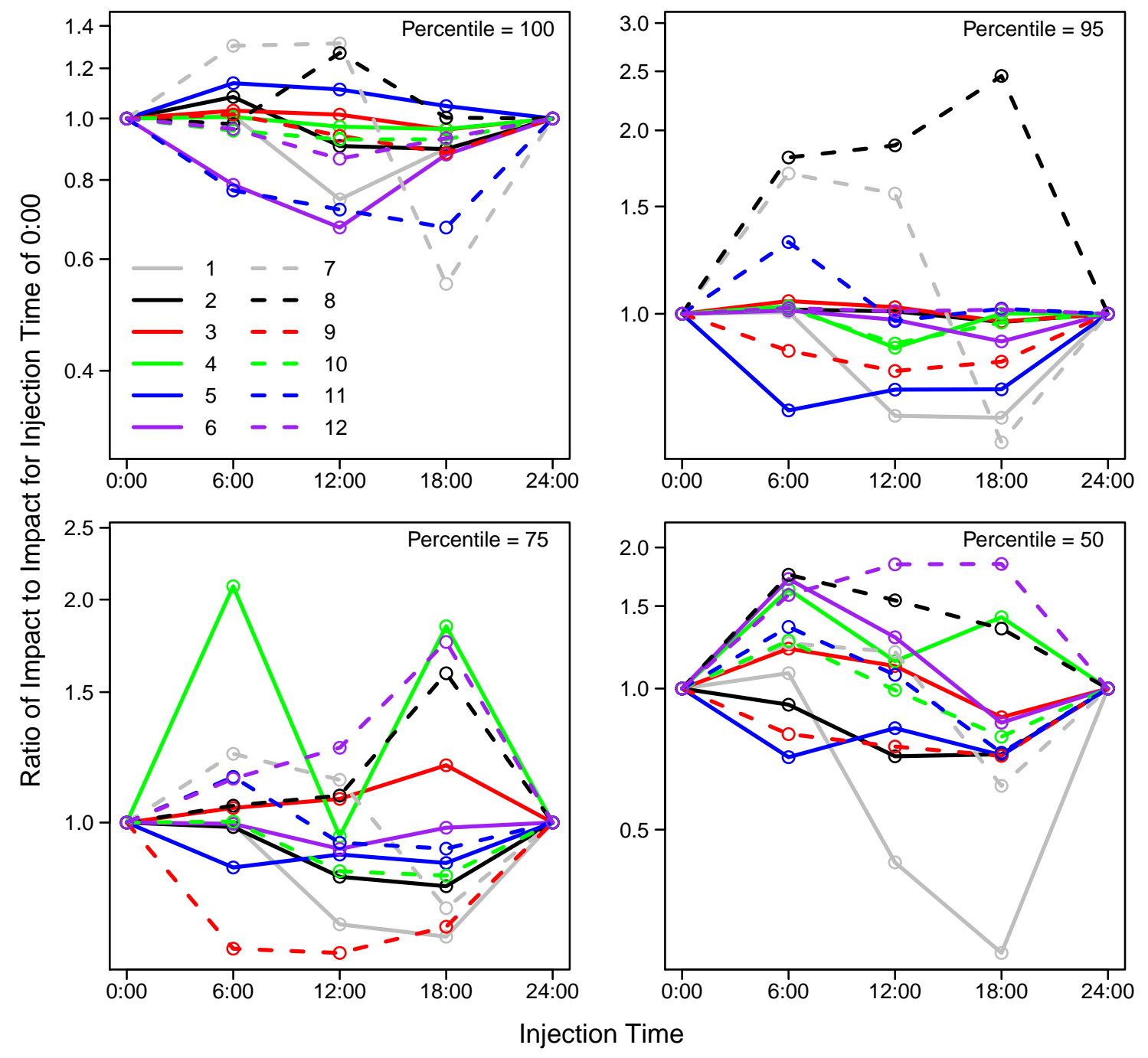

Figure 5.5. Relative impacts for the 12 networks for different injection times, for a dose level of $0.0001 \mathrm{mg}$ and for injection nodes associated with different percentile impacts. Note that the vertical scale is logarithmic. 


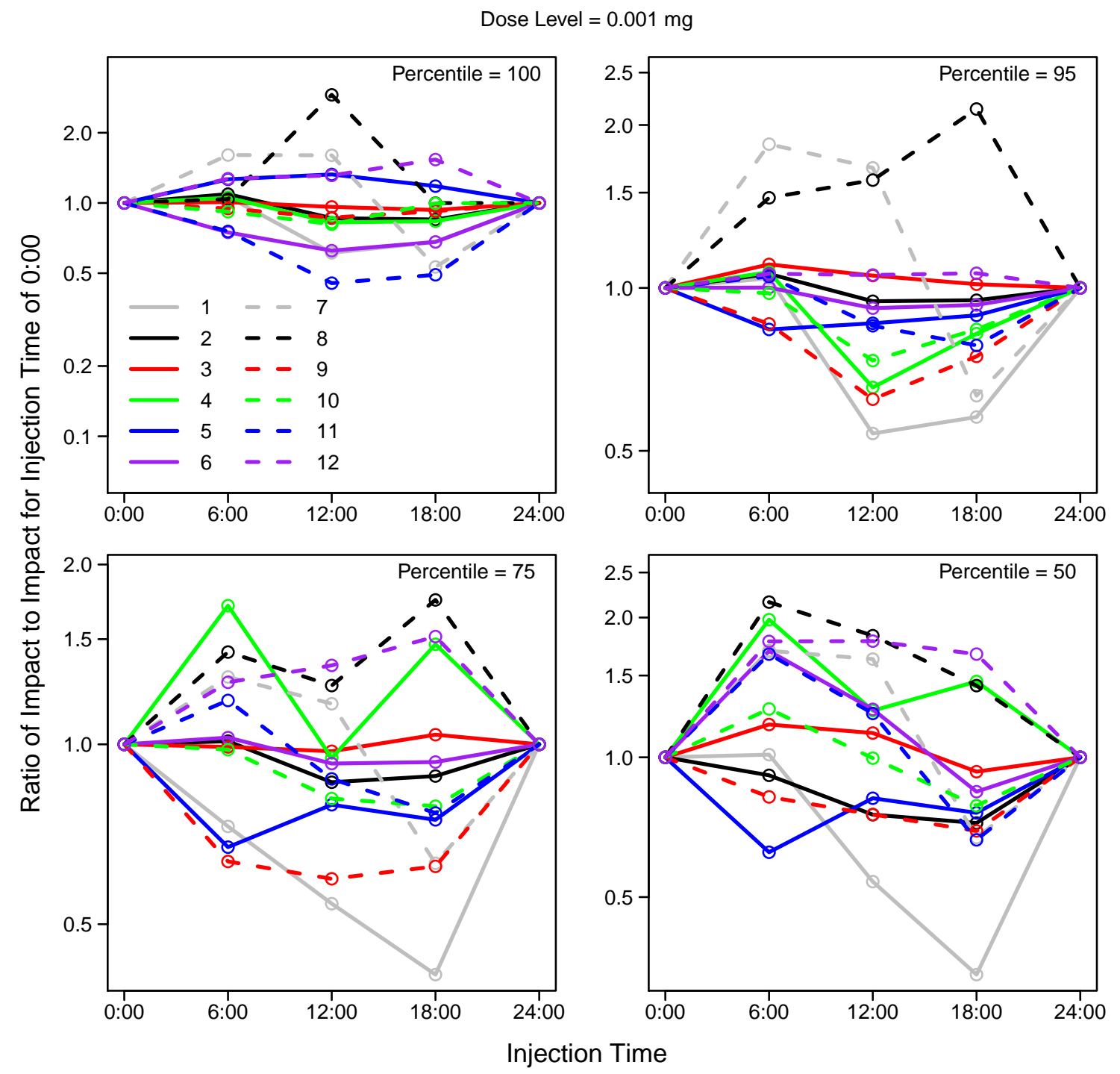

Figure 5.6. Relative impacts for the 12 networks for different injection times, for a dose level of $0.001 \mathrm{mg}$ and for injection nodes associated with different percentile impacts. Note that the vertical scale is logarithmic. 
Dose Level $=0.01 \mathrm{mg}$

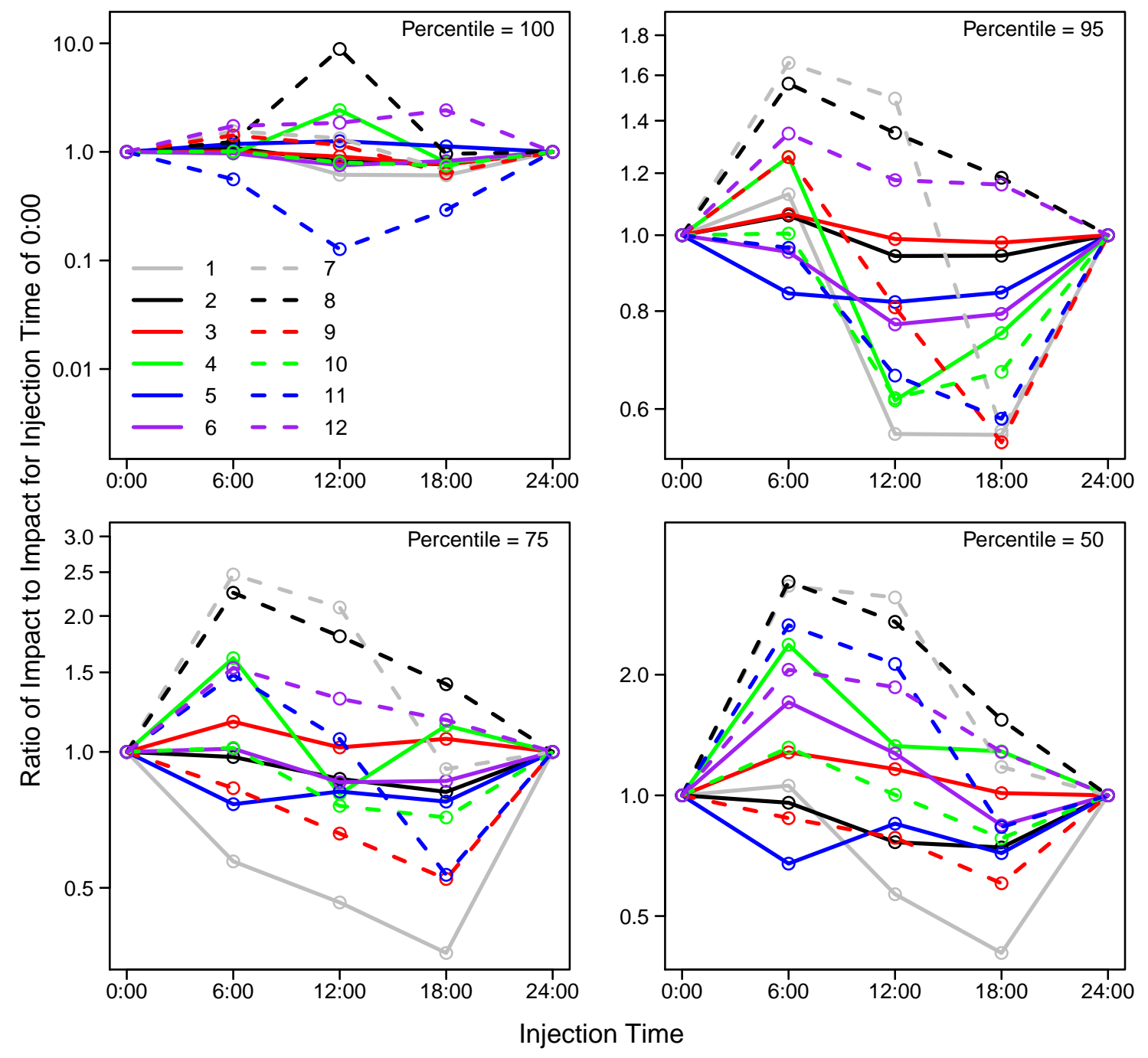

Figure 5.7. Relative impacts for the 12 networks for different injection times, for a dose level of $0.01 \mathrm{mg}$ and for injection nodes associated with different percentile impacts. Note that the vertical scale is logarithmic. 
Dose Level $=0.1 \mathrm{mg}$

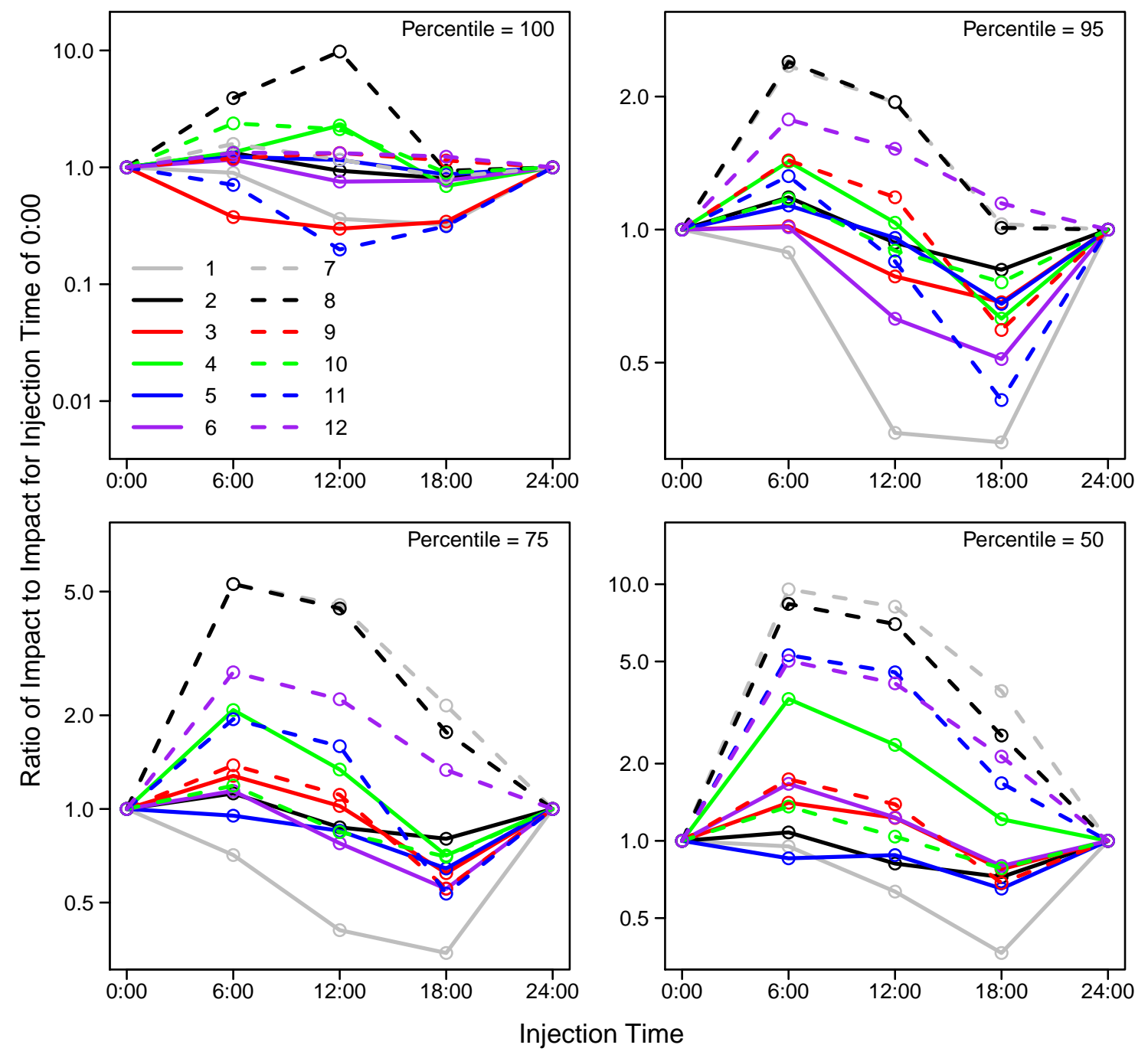

Figure 5.8. Relative impacts for the 12 networks for different injection times, for a dose level of $0.1 \mathrm{mg}$ and for injection nodes associated with different percentile impacts. Note that the vertical scale is logarithmic. 
Dose Level $=1.0 \mathrm{mg}$

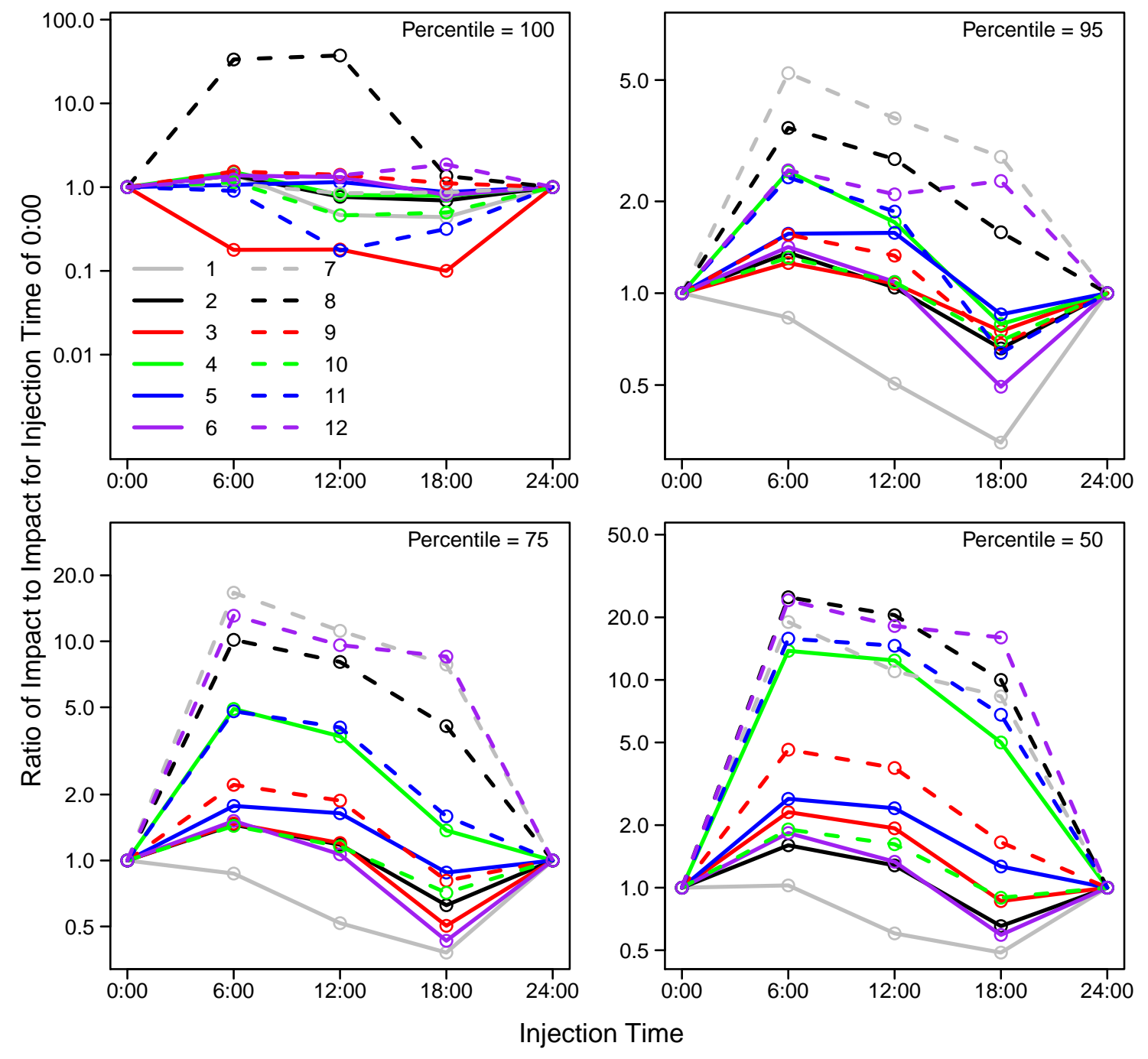

Figure 5.9. Relative impacts for the 12 networks for different injection times, for a dose level of $1 \mathrm{mg}$ and for injection nodes associated with different percentile impacts. Note that the vertical scale is logarithmic. 


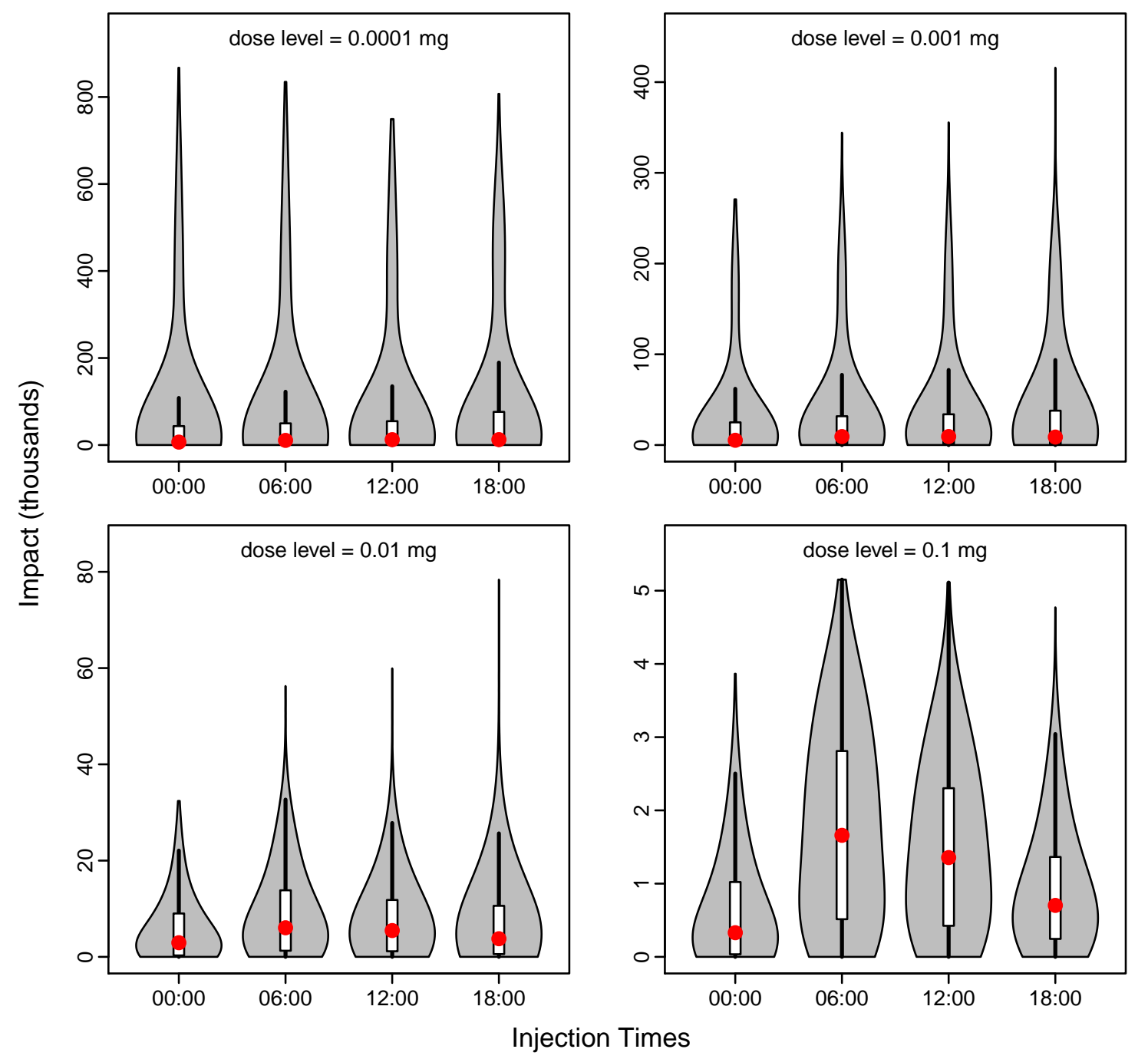

Figure 5.10. Distributions of impacts for Network 12 across all injection nodes for different injection times, for dose levels of $0.0001,0.001,0.01$, and $0.1 \mathrm{mg}$. 


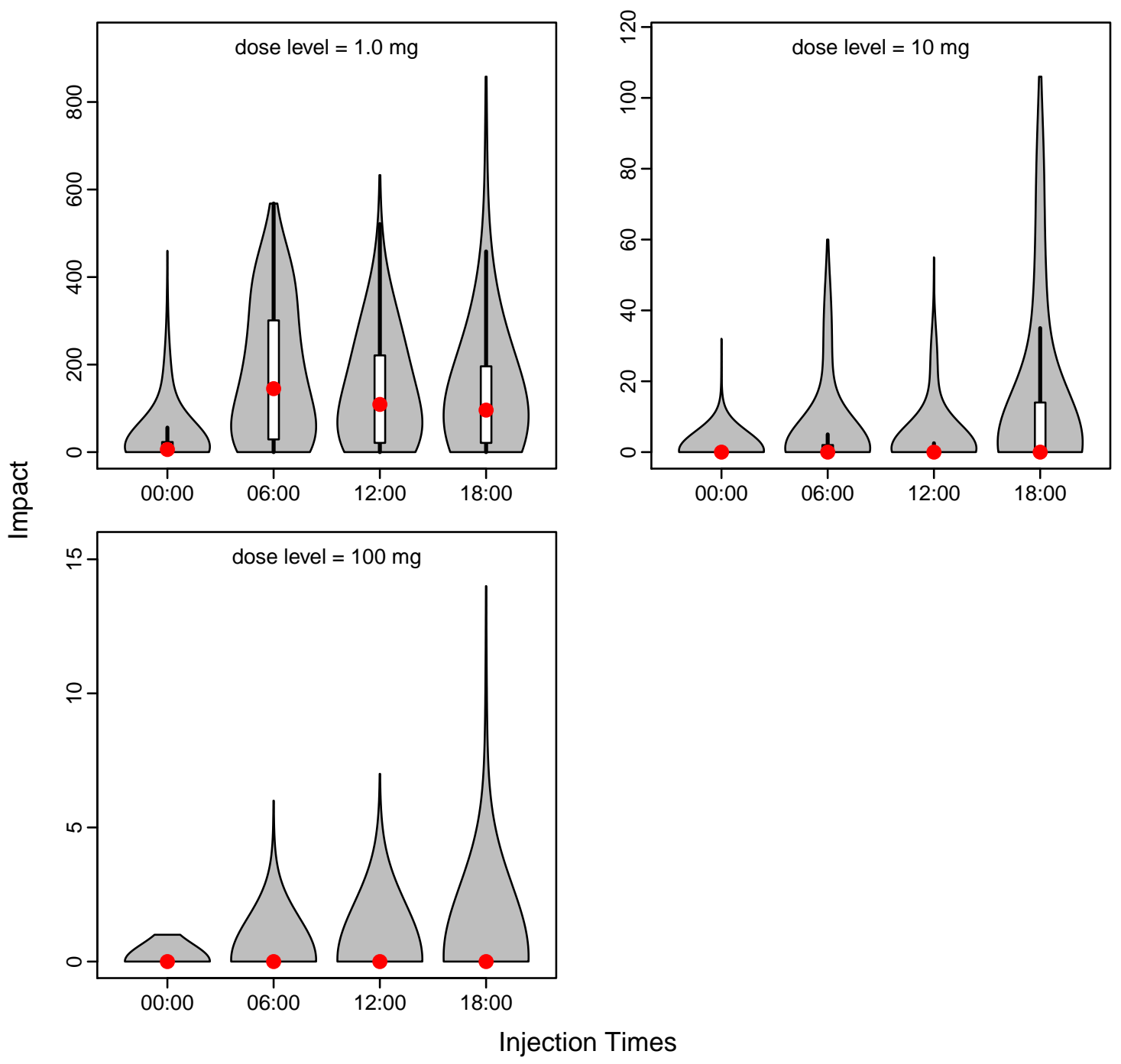

Figure 5.11. Distributions of impacts for Network 12 across all injection nodes for different injection times, for dose levels of 1,10 , and $100 \mathrm{mg}$. 


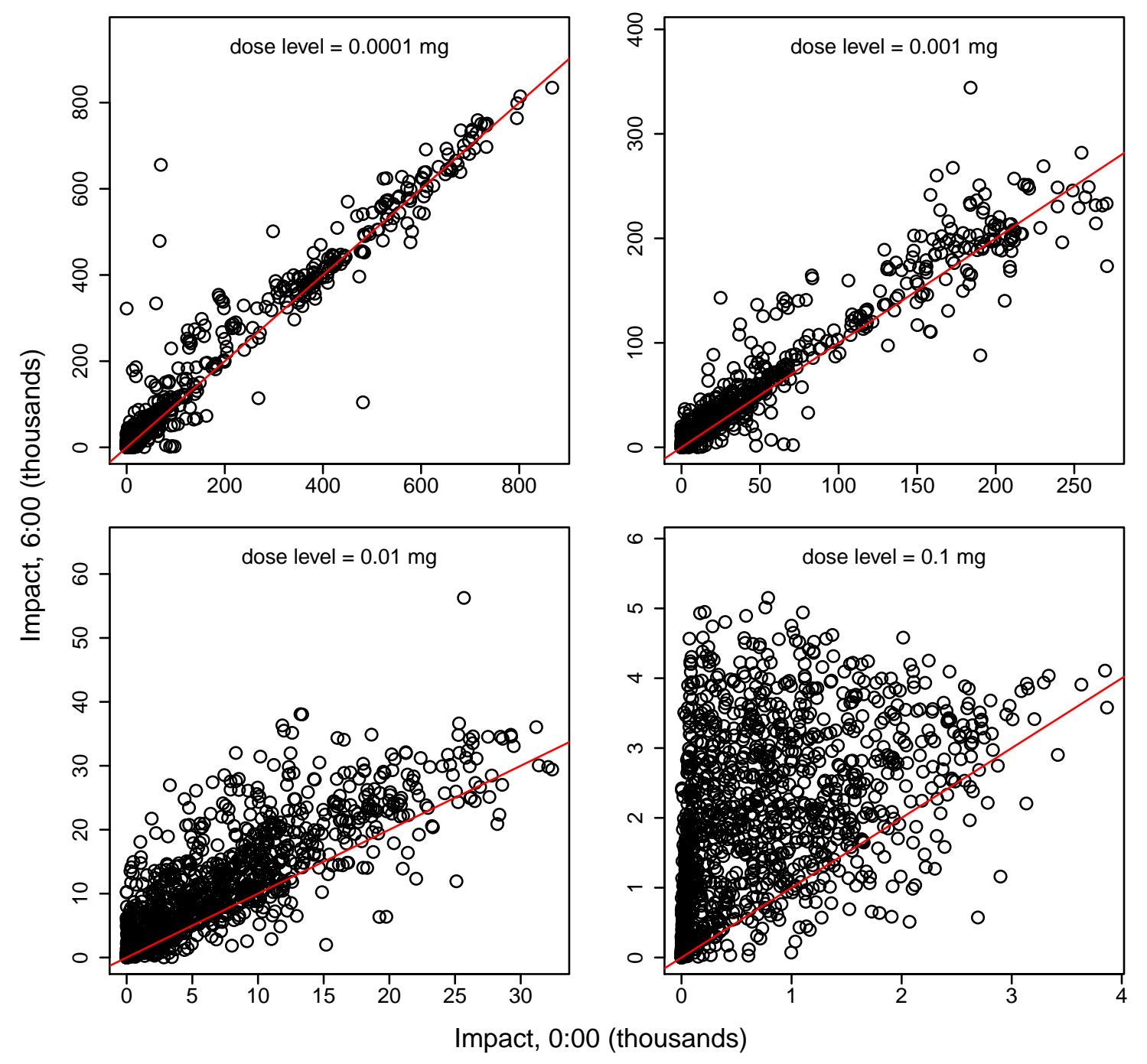

Figure 5.12. Impacts for Network 12 for injection times of 0:00 and 6:00, for dose levels of $0.0001,0.001,0.01$, and $0.1 \mathrm{mg}$. The red lines have a slope of 1 . Each point corresponds to one injection node. 


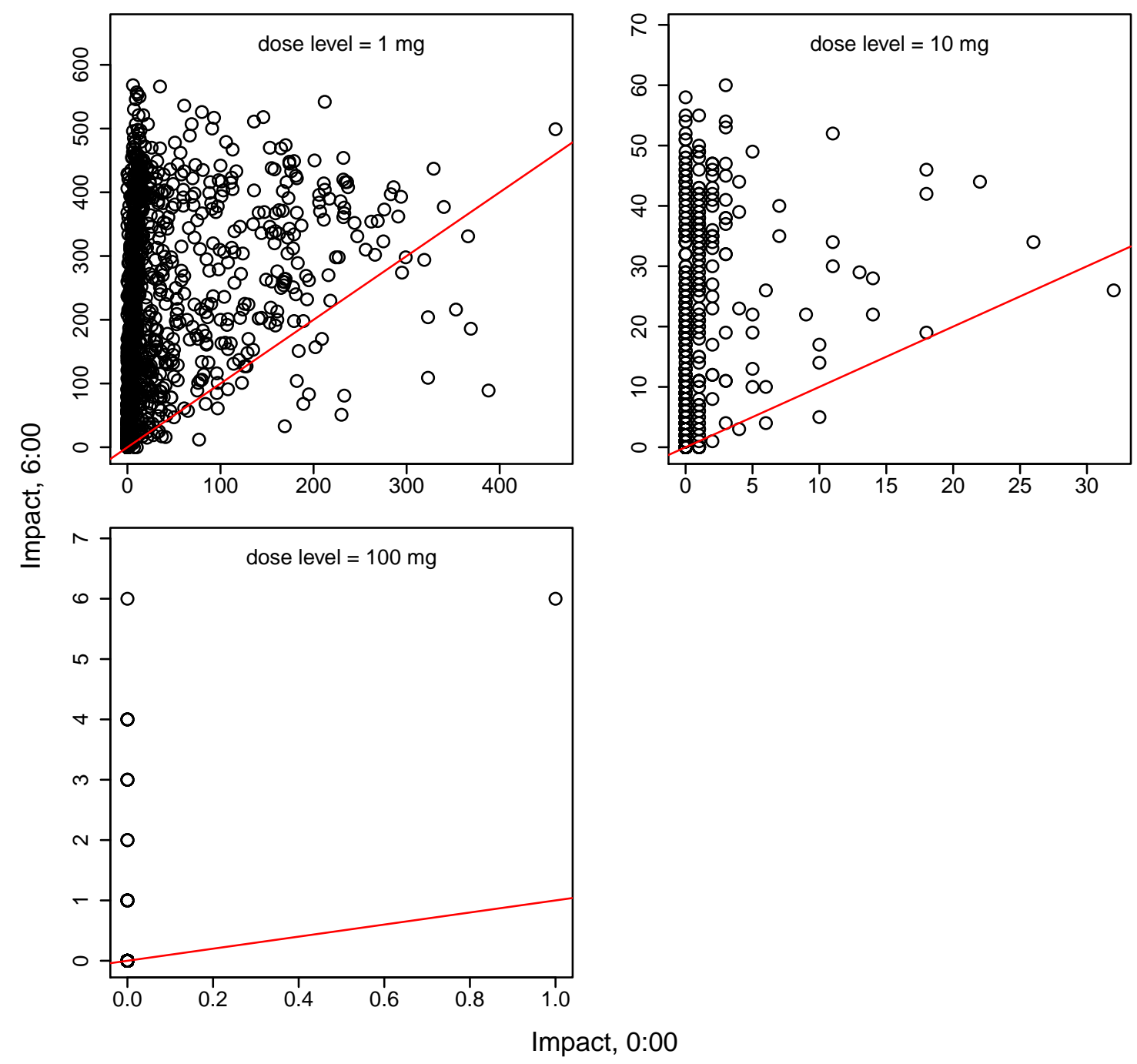

Figure 5.13. Impacts for injection times of 0:00 and 6:00, for dose levels of 1, 10, and $100 \mathrm{mg}$. The red lines have a slope of 1 . Each point corresponds to one injection node. 


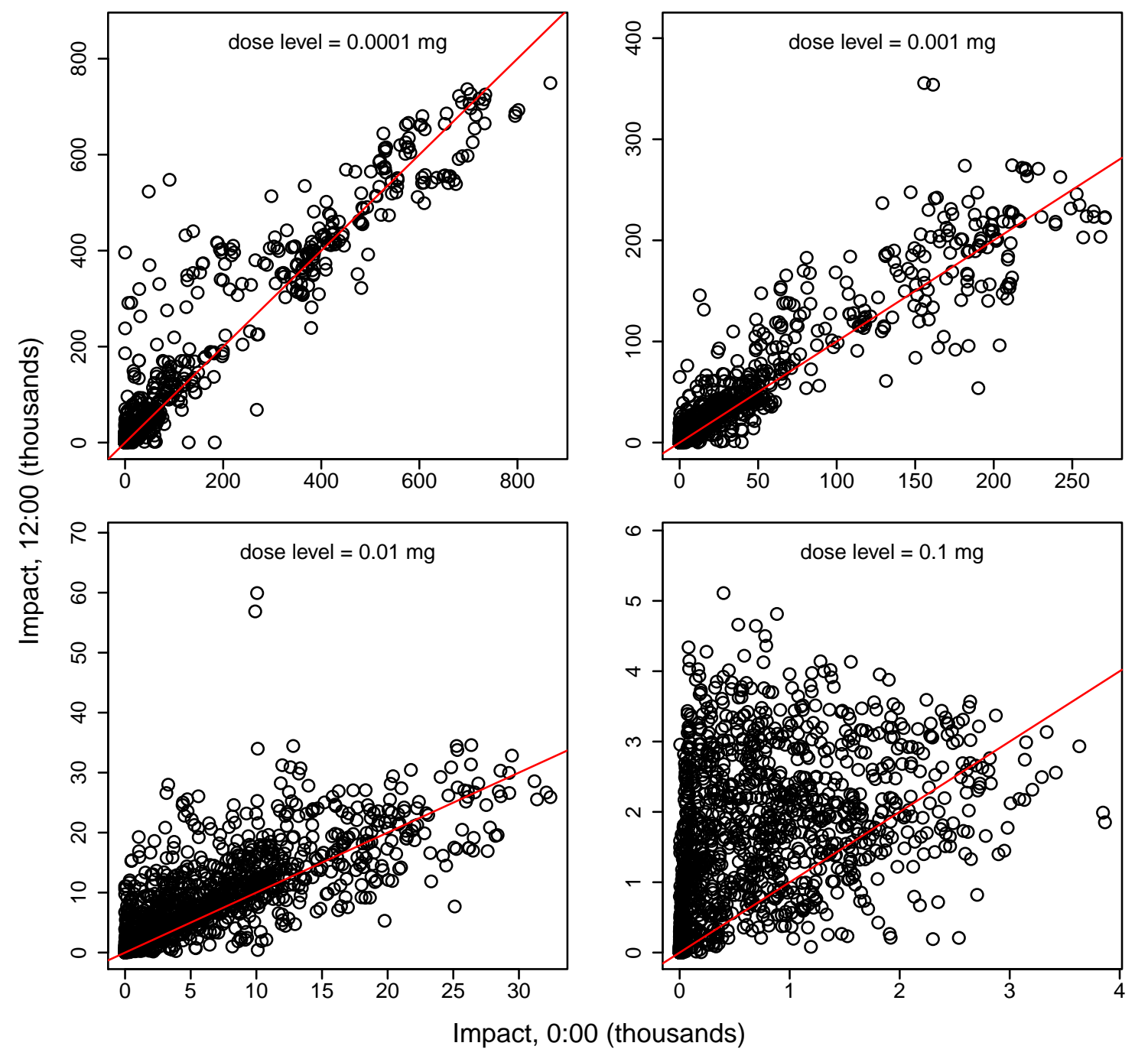

Figure 5.14. Impacts for Network 12 for injection times of 0:00 and 12:00, for dose levels of $0.0001,0.001,0.01$, and $0.1 \mathrm{mg}$. The red lines have a slope of 1 . Each point corresponds to one injection node. 


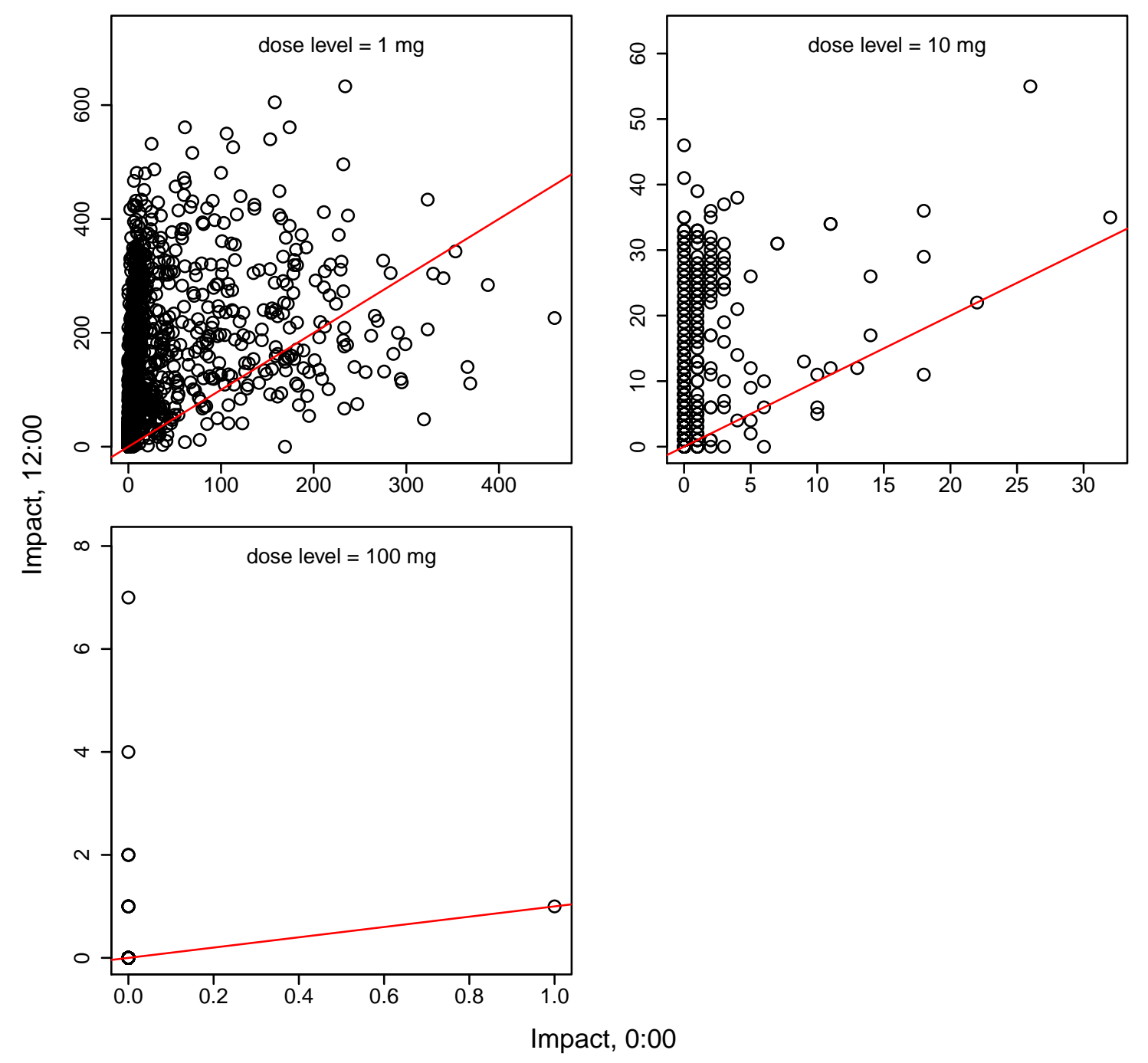

Figure 5.15. Impacts for Network 12 for injection times of 0:00 and 12:00, for dose levels of 1,10 , and $100 \mathrm{mg}$. The red lines have a slope of 1 . Each point corresponds to one injection node. 


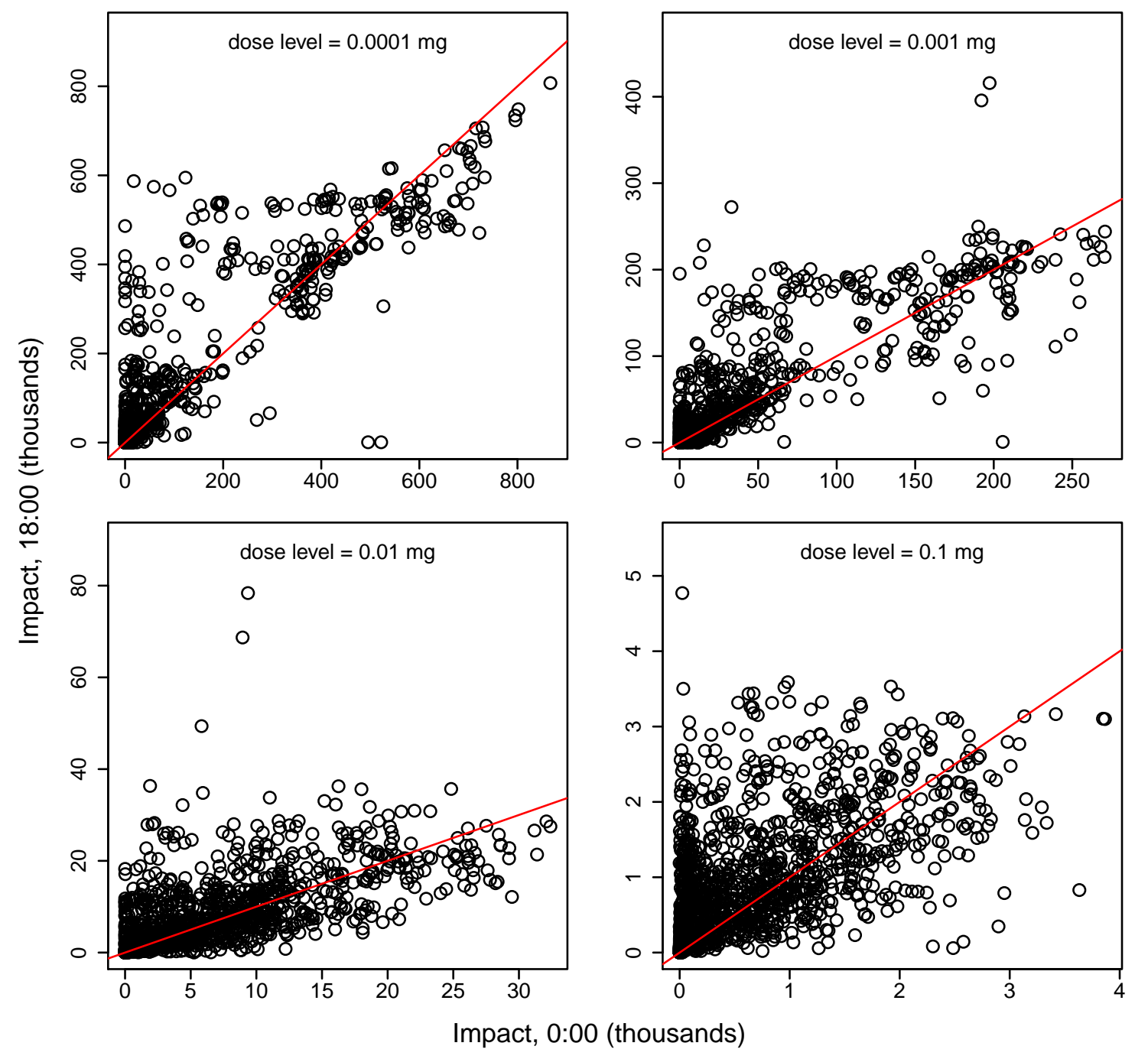

Figure 5.16. Impacts for Network 12 for injection times of 0:00 and 18:00, for dose levels of $0.0001,0.001,0.01$, and $0.1 \mathrm{mg}$. The red lines have a slope of 1 . Each point corresponds to one injection node. 


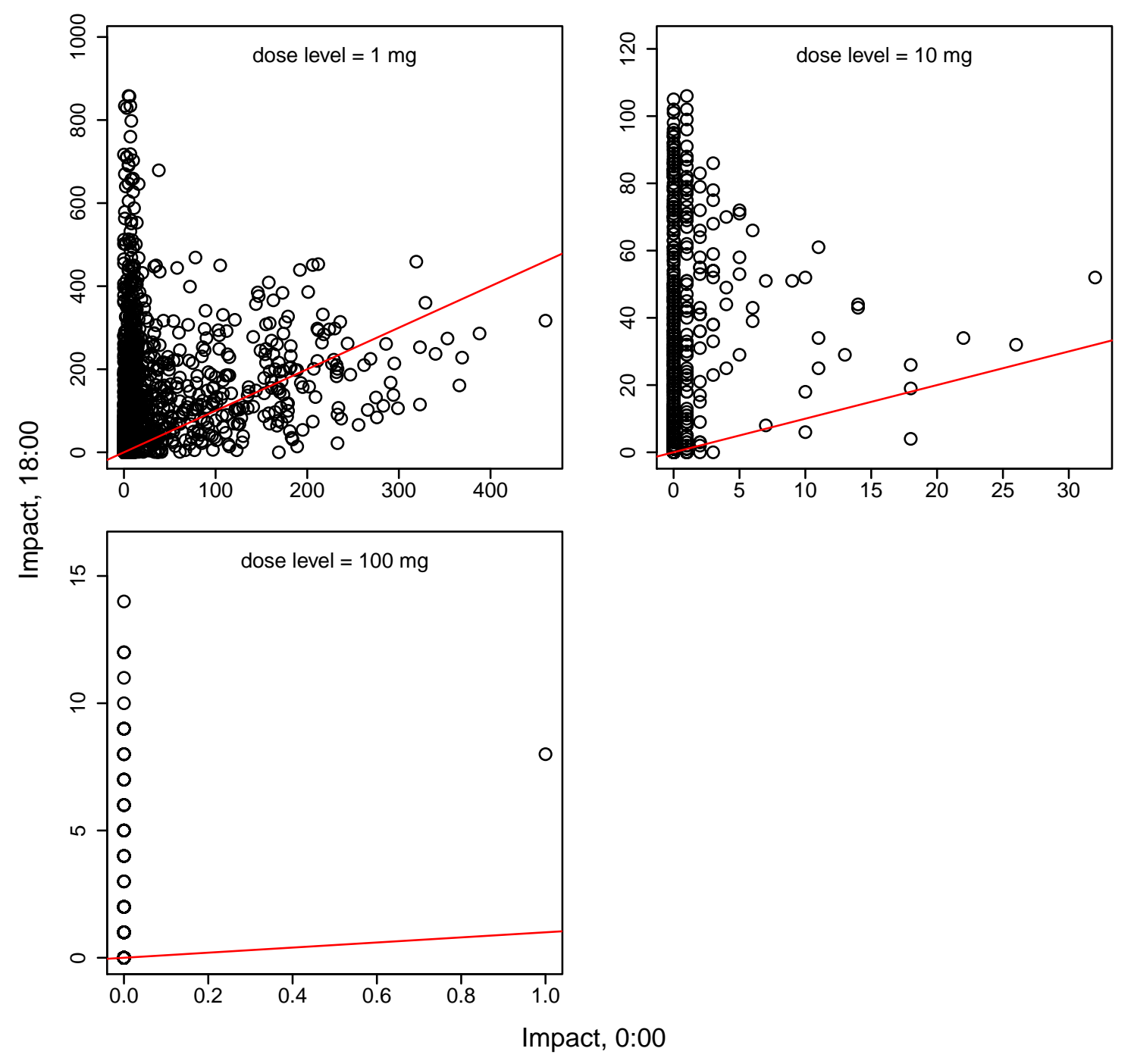

Figure 5.17. Impacts for Network 12 for injection times of 0:00 and 18:00, for dose levels of 1,10 , and $100 \mathrm{mg}$. The red lines have a slope of 1 . Each point corresponds to one injection node. 


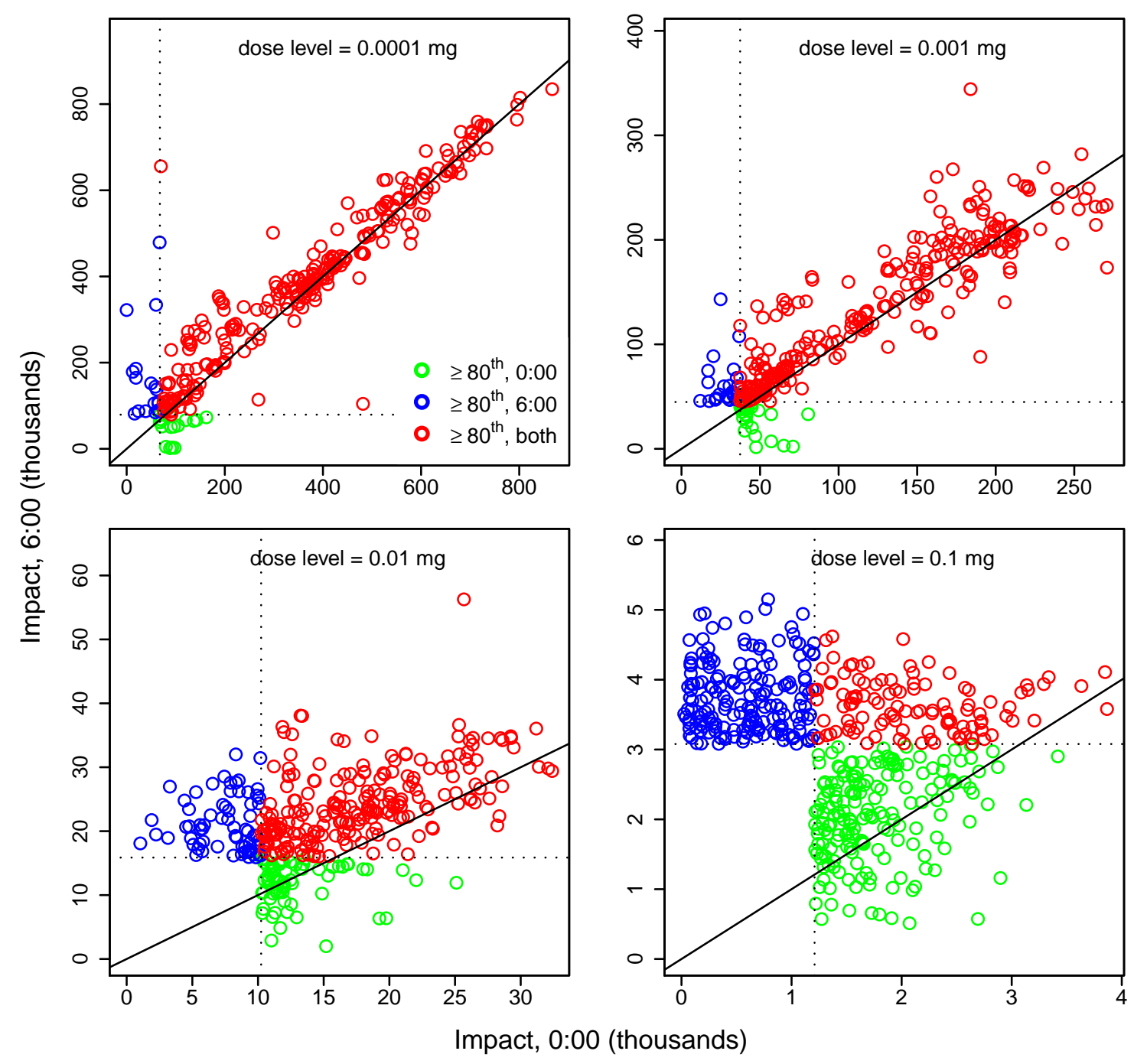

Figure 5.18. Impacts for Network 12 that are at or above the $80^{\text {th }}$ percentile level for an injection time of 6:00 and/or an injection time of 0:00, for dose levels of $0.0001,0.001,0.01$, and $0.1 \mathrm{mg}$. The dotted lines indicate $80^{\text {th }}$ percentile impacts. The black lines have a slope of 1. Each point corresponds to one injection node. 


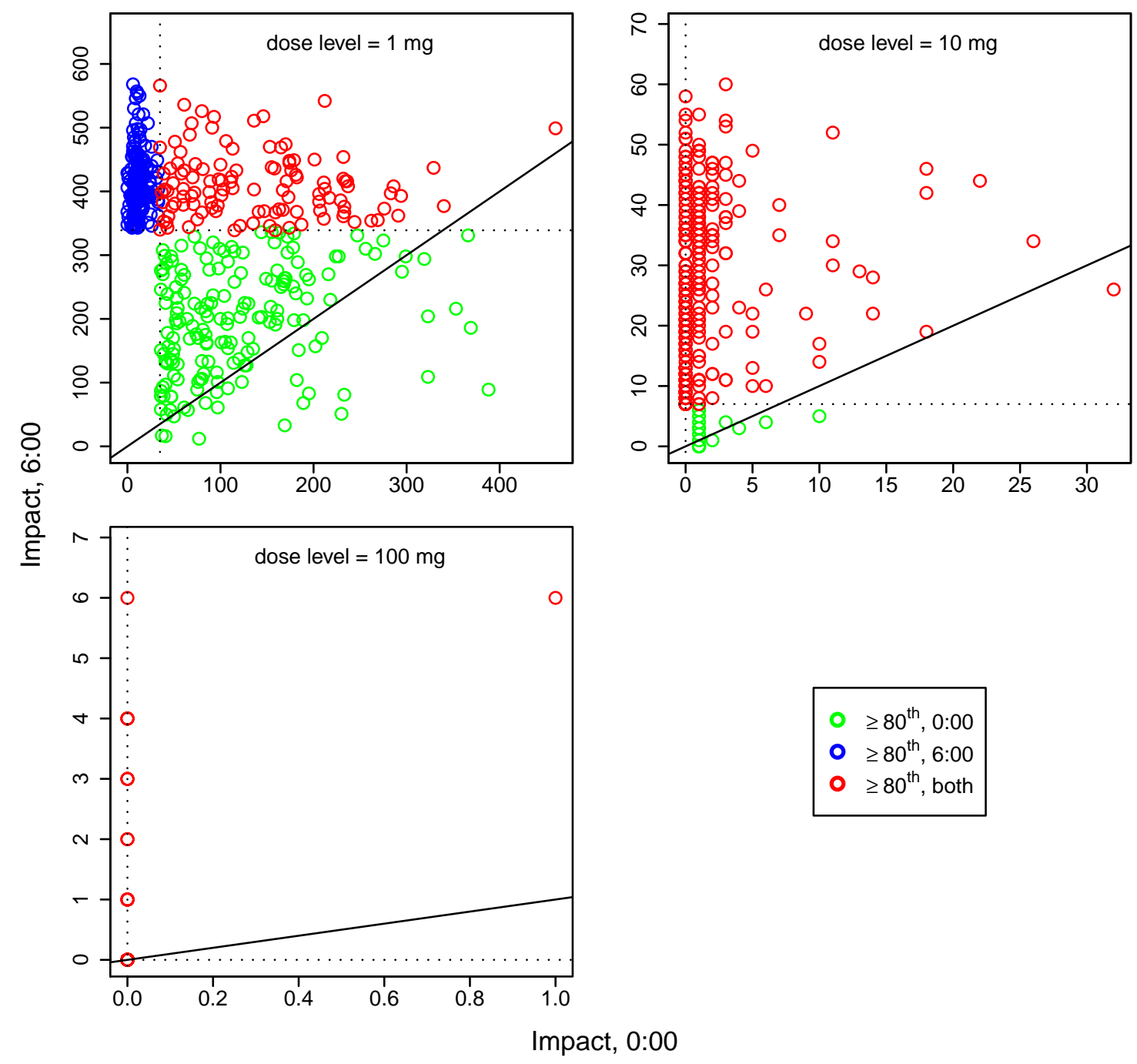

Figure 5.19. Impacts for Network 12 that are at or above the $80^{\text {th }}$ percentile level for an injection time of 6:00 and/or an injection time of 0:00, for dose levels of 1, 10, and $100 \mathrm{mg}$. The dotted lines indicate $80^{\text {th }}$ percentile impacts. The black lines have a slope of 1 . Each point corresponds to one injection node. 


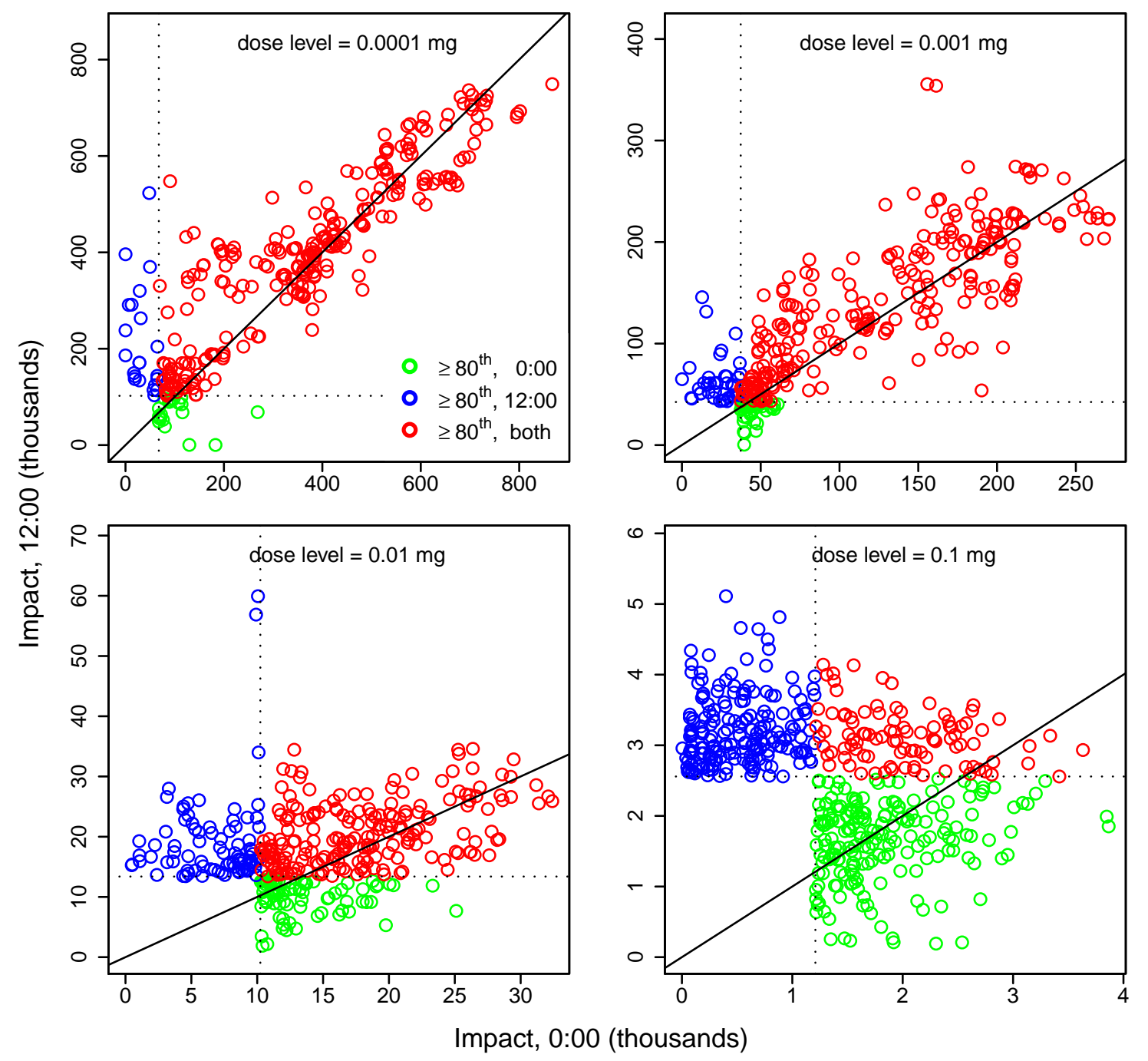

Figure 5.20. Impacts for Network 12 that are at or above the $80^{\text {th }}$ percentile level for an injection time of 12:00 and/or an injection time of 0:00, for dose levels of $0.0001,0.001,0.01$, and $0.1 \mathrm{mg}$. The dotted lines indicate $80^{\text {th }}$ percentile impacts. The black lines have a slope of 1. Each point corresponds to one injection node. 


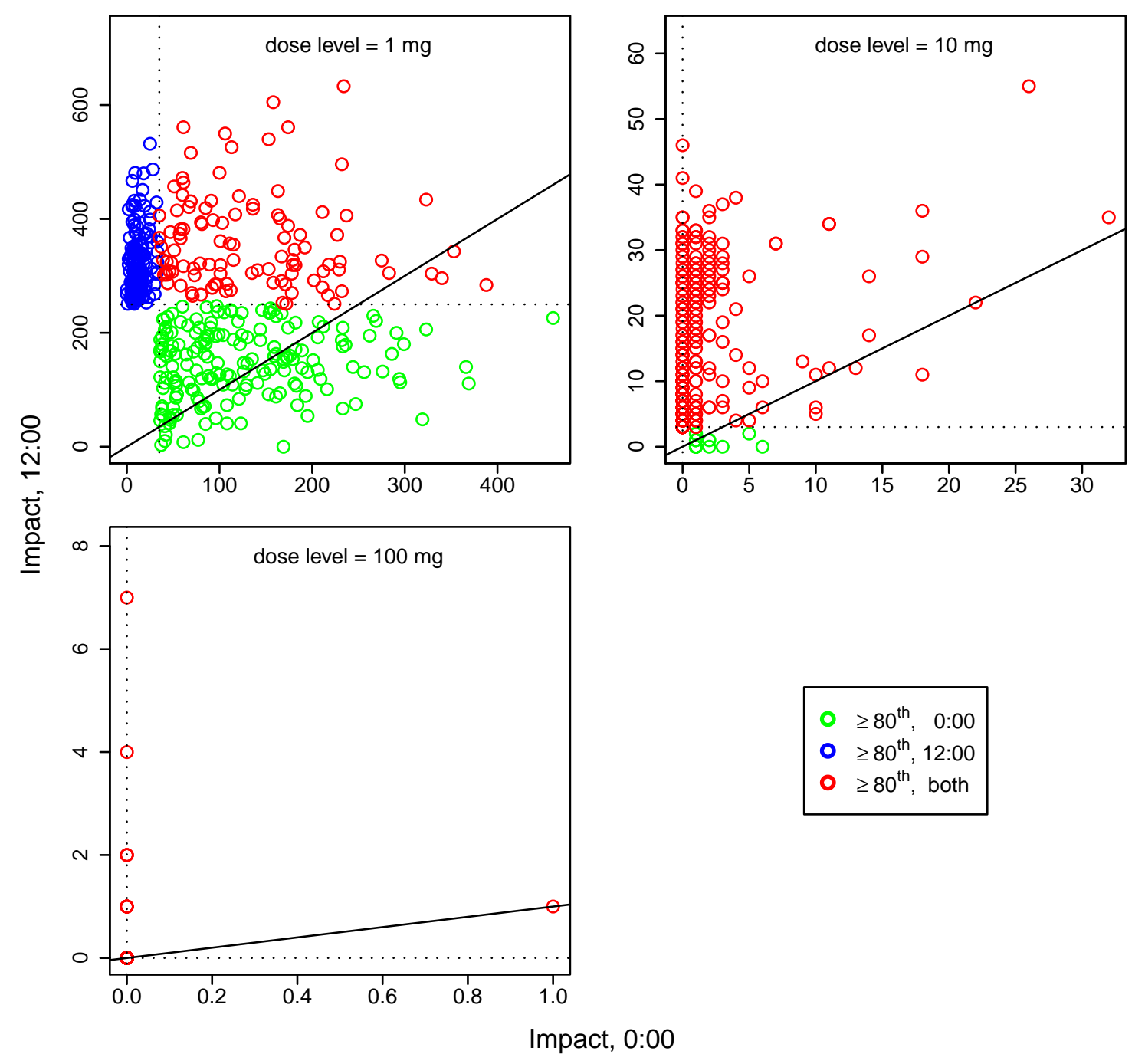

Figure 5.21. Impacts for Network 12 that are at or above the $80^{\text {th }}$ percentile level for an injection time of 12:00 and/or an injection time of 0:00, for dose levels of 1, 10, and $100 \mathrm{mg}$. The dotted lines indicate $80^{\text {th }}$ percentile impacts. The black lines have a slope of 1 . Each point corresponds to one injection node. 


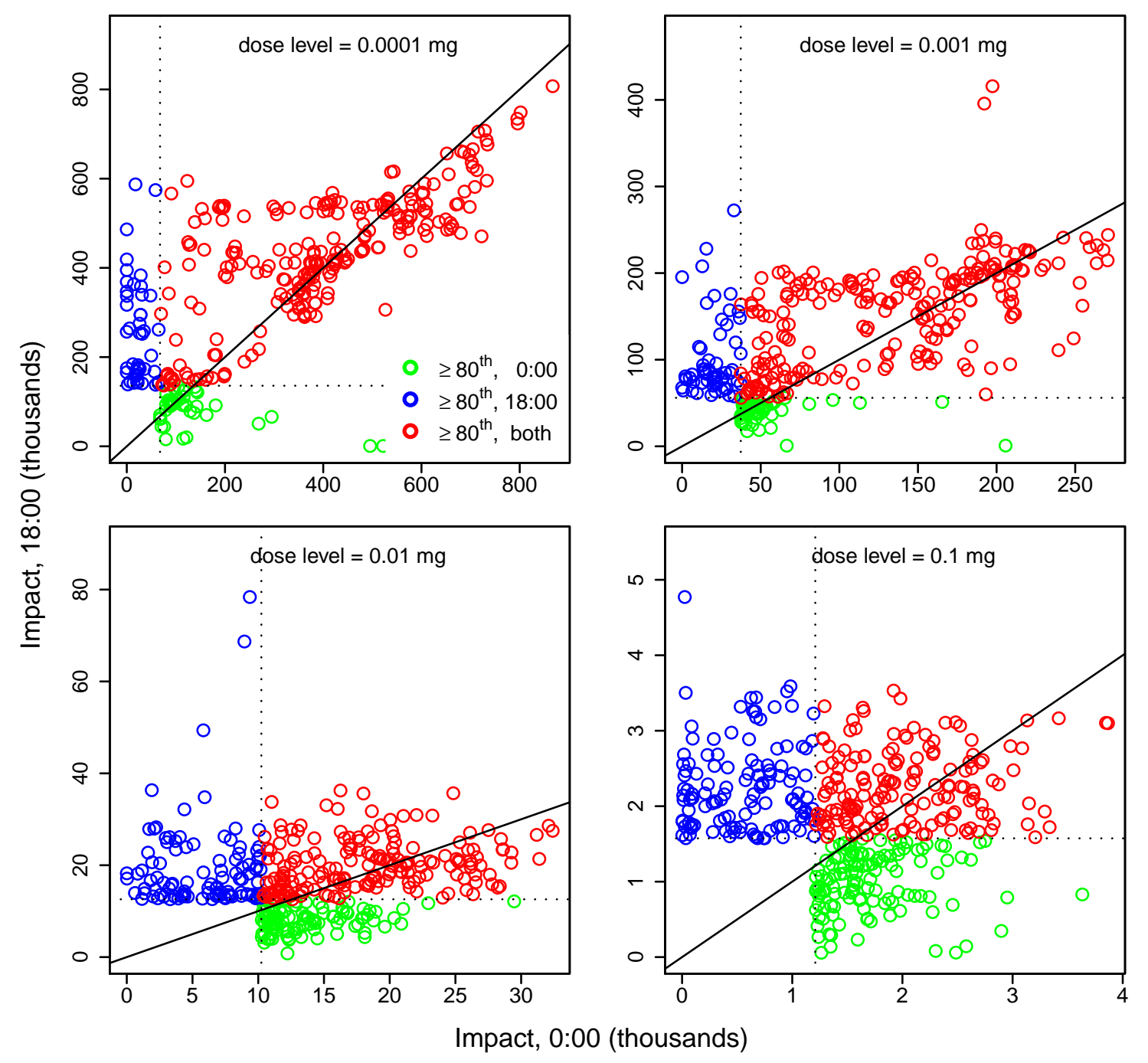

Figure 5.22. Impacts for Network 12 that are at or above the $80^{\text {th }}$ percentile level for an injection time of 18:00 and/or an injection time of 0:00, for dose levels of $0.0001,0.001,0.01$, and $0.1 \mathrm{mg}$. The dotted lines indicate $80^{\text {th }}$ percentile impacts. The black lines have a slope of 1. Each point corresponds to one injection node. 


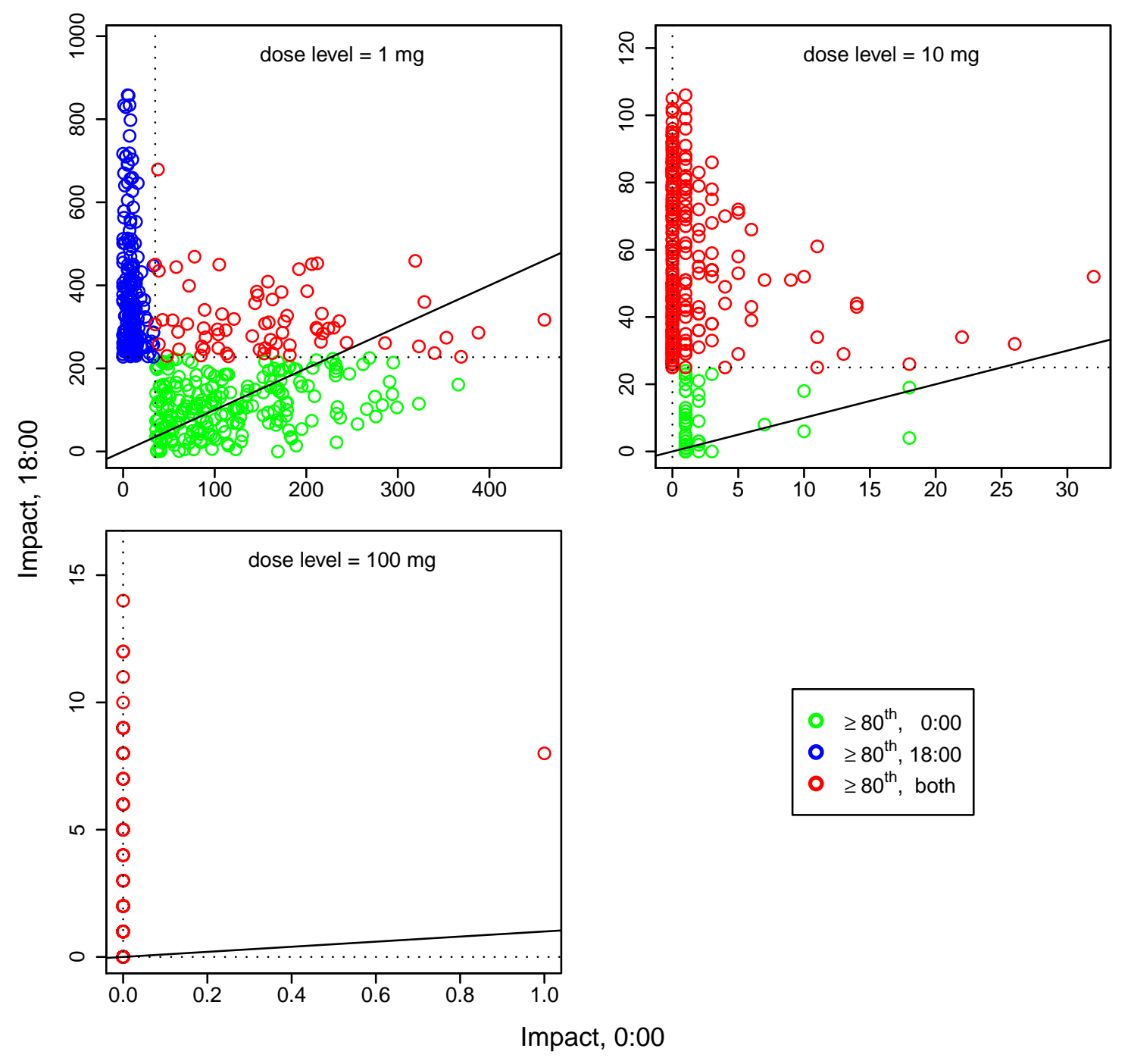

Figure 5.23. Impacts for Network 12 that are at or above the $80^{\text {th }}$ percentile level for an injection time of 18:00 and/or an injection time of 0:00, for dose levels of 1, 10, and $100 \mathrm{mg}$. The dotted lines indicate $80^{\text {th }}$ percentile impacts. The black lines have a slope of 1 . Each point corresponds to one injection node. 


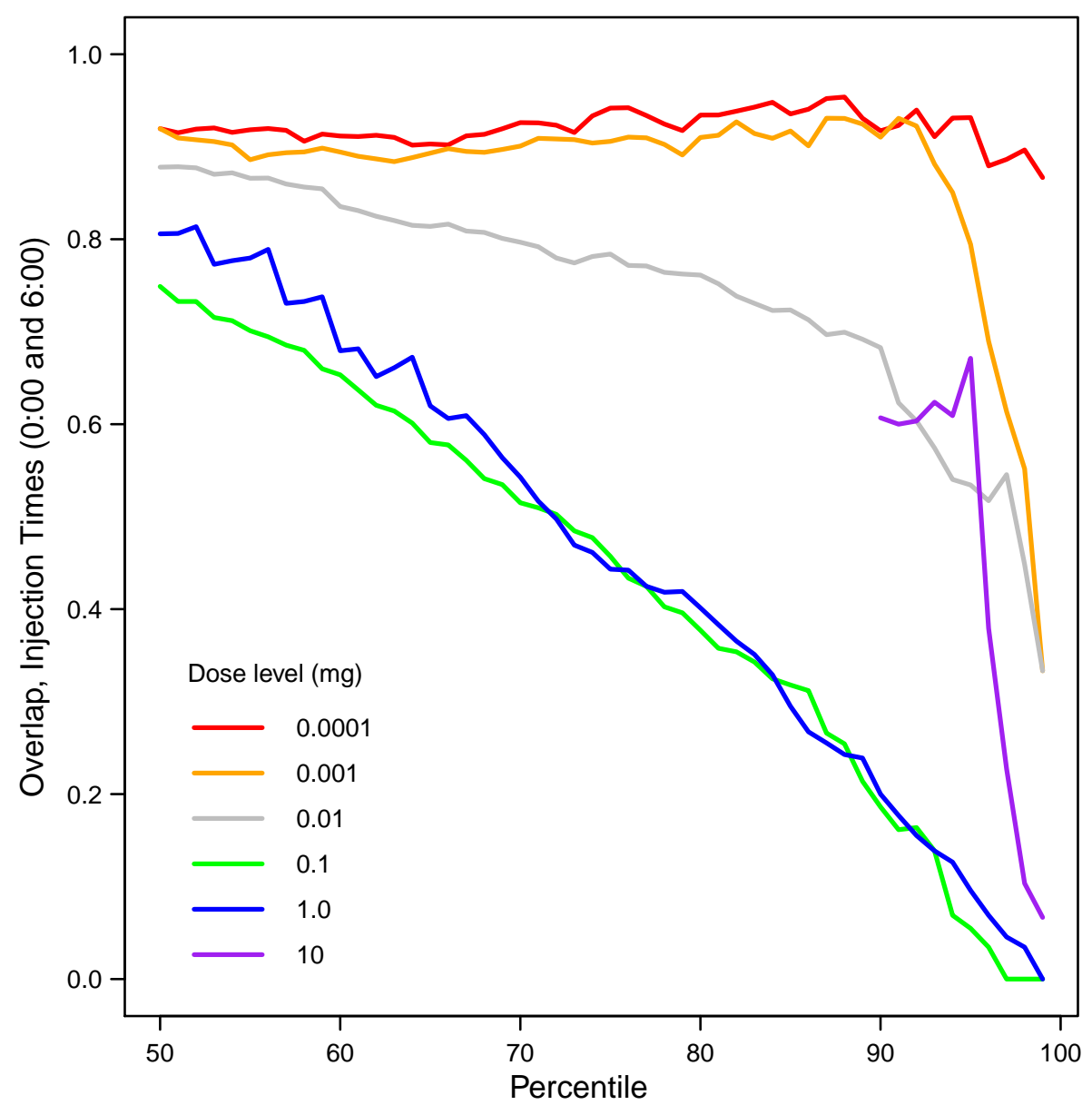

Figure 5.24. Overlap of high percentile injection nodes for Network 12 for an injection time of 6:00 with those for an injection time of 0:00 for different dose levels. 


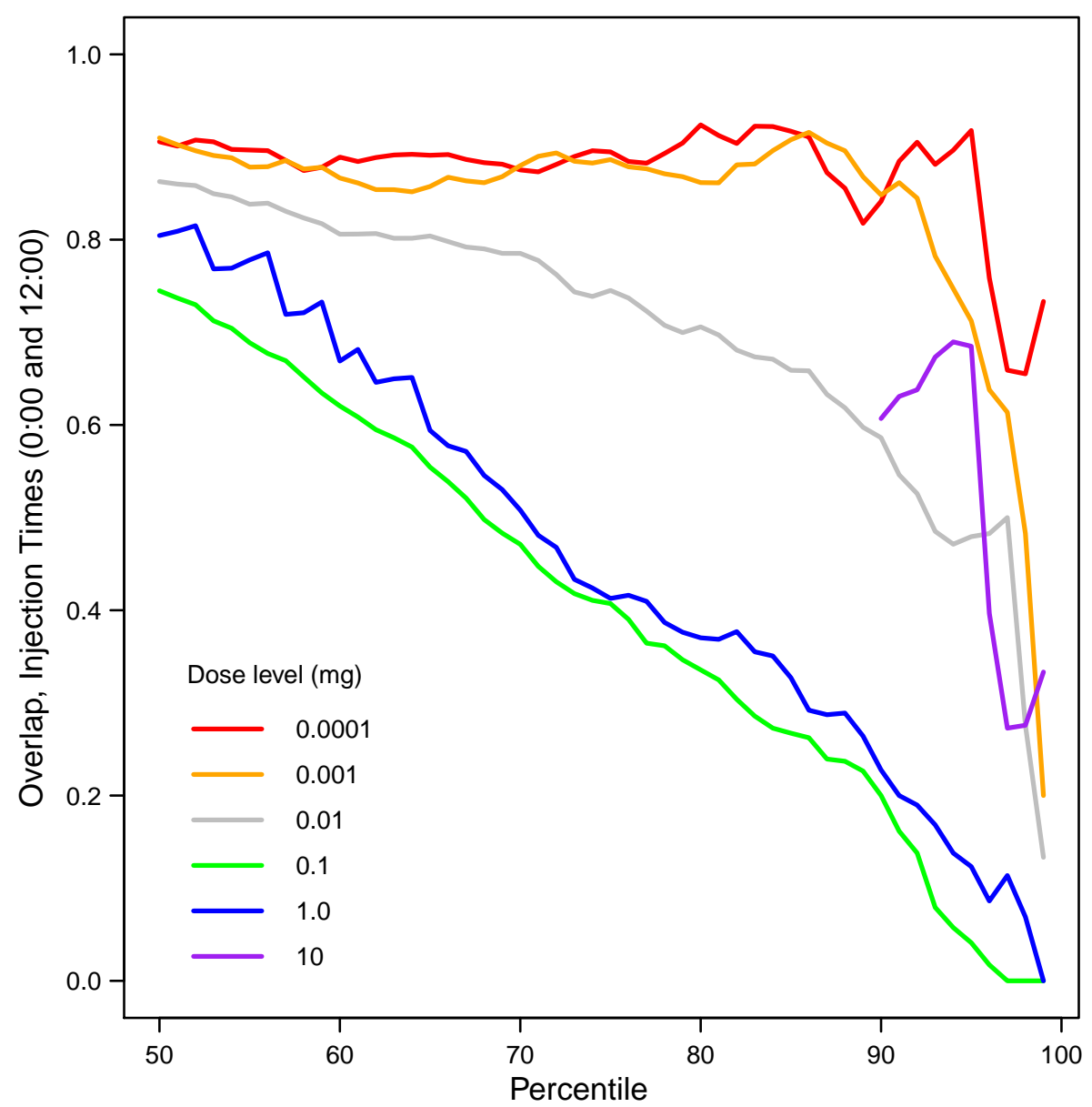

Figure 5.25. Overlap of high percentile injection nodes for Network 12 for an injection time of 12:00 with those for an injection time of 0:00 for different dose levels. 


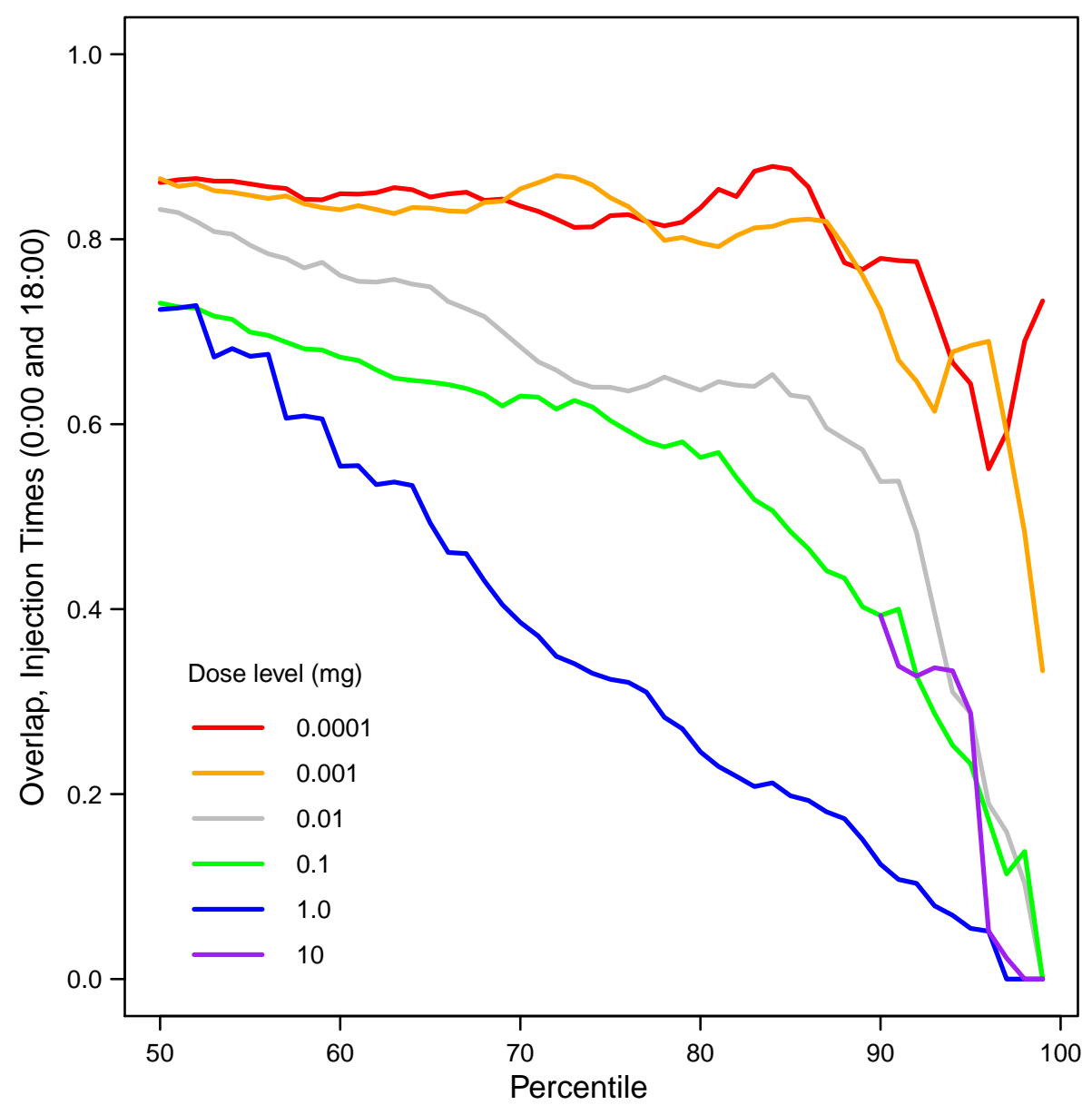

Figure 5.26. Overlap of high percentile injection nodes for Network 12 for an injection time of 18:00 with those for an injection time of 0:00 for different dose levels. 

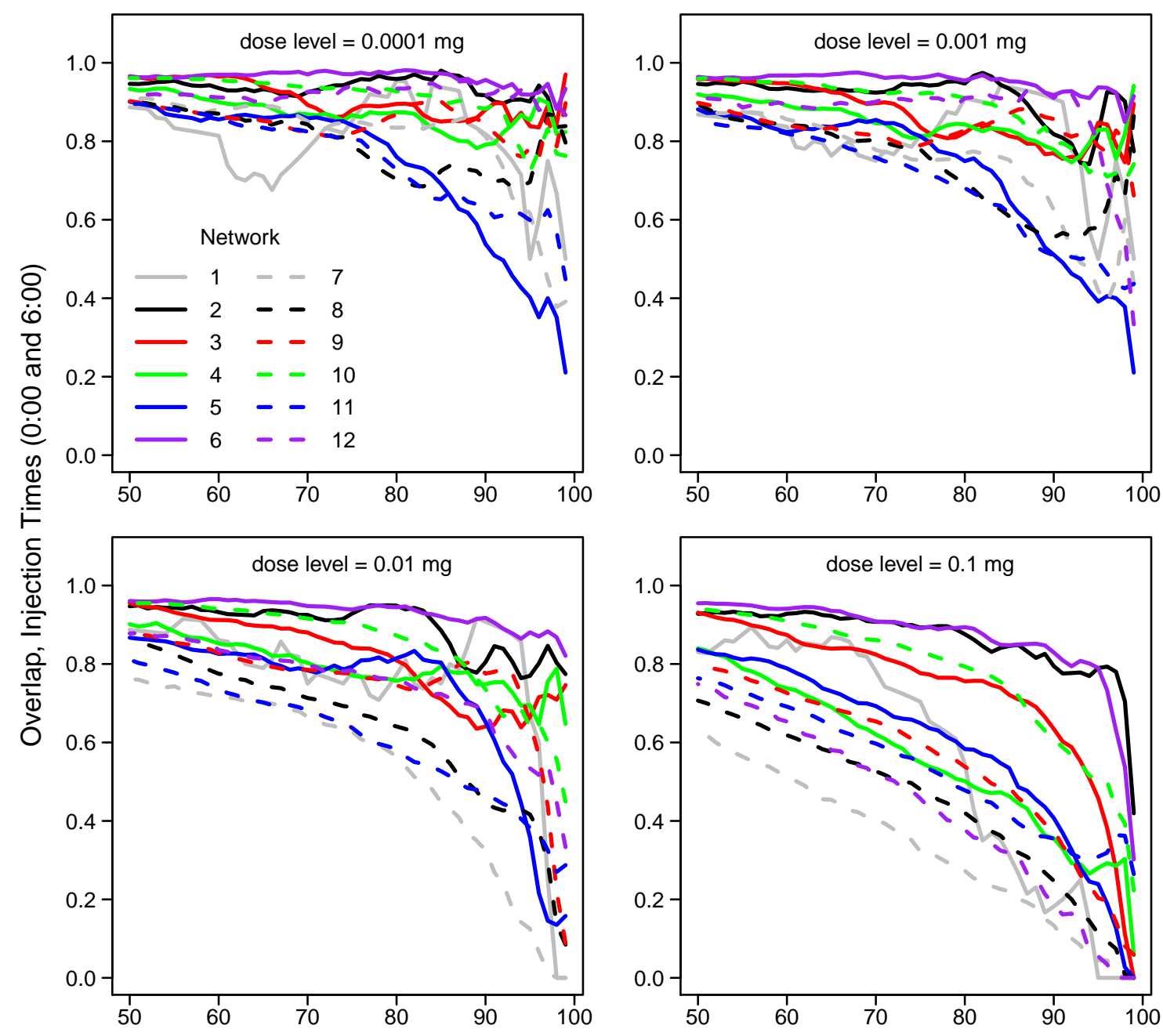

Percentile

Figure 5.27. Overlap of high percentile injection nodes for the networks for an injection time of 6:00 with those for an injection time of 0:00, for dose levels of $0.0001,0.001,0.01$, and 0.1 mg. 

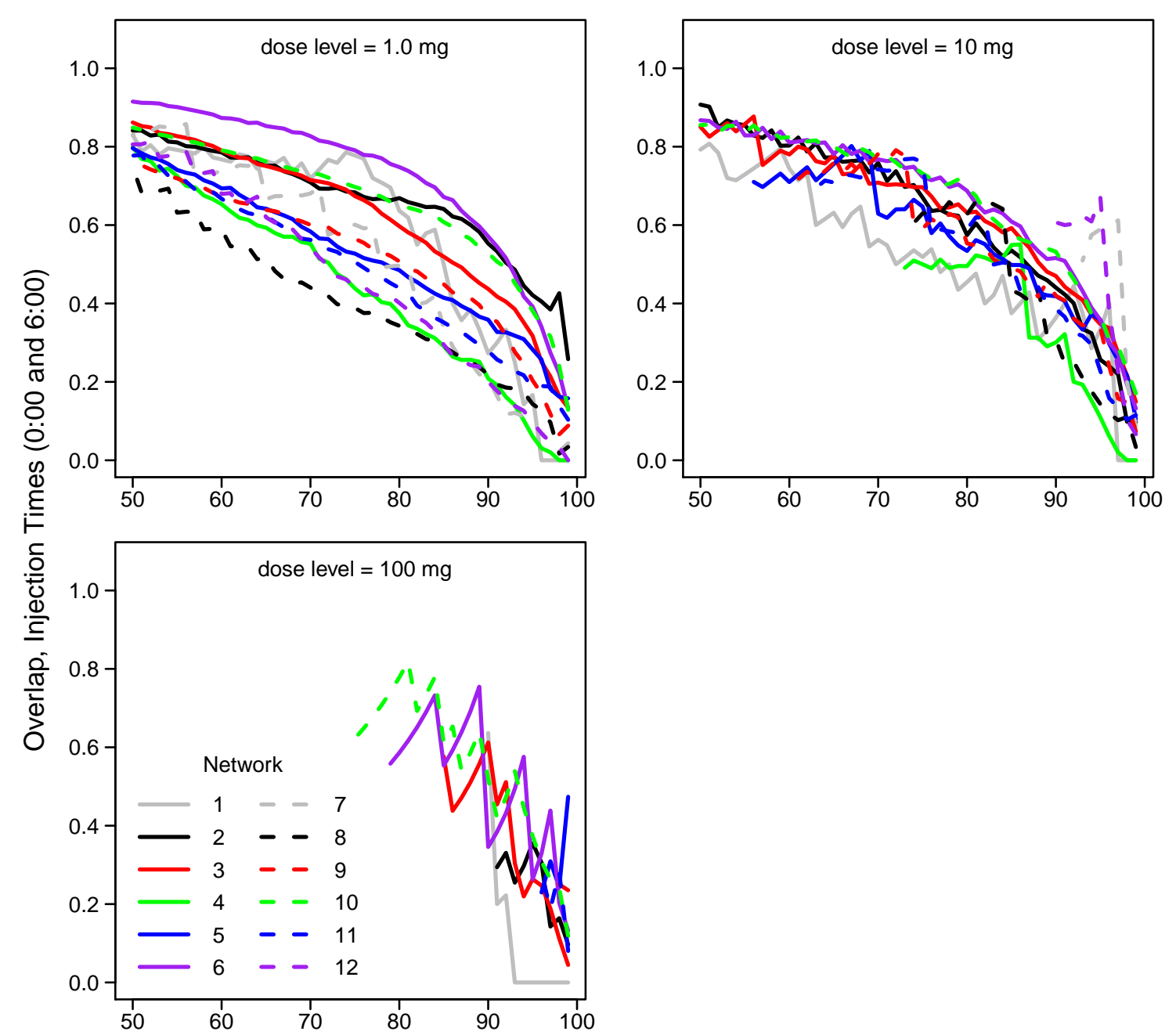

Percentile

Figure 5.28. Overlap of high percentile injection nodes for the networks for an injection time of 6:00 with those for an injection time of $0: 00$, for dose levels of 1,10 , and $100 \mathrm{mg}$. 

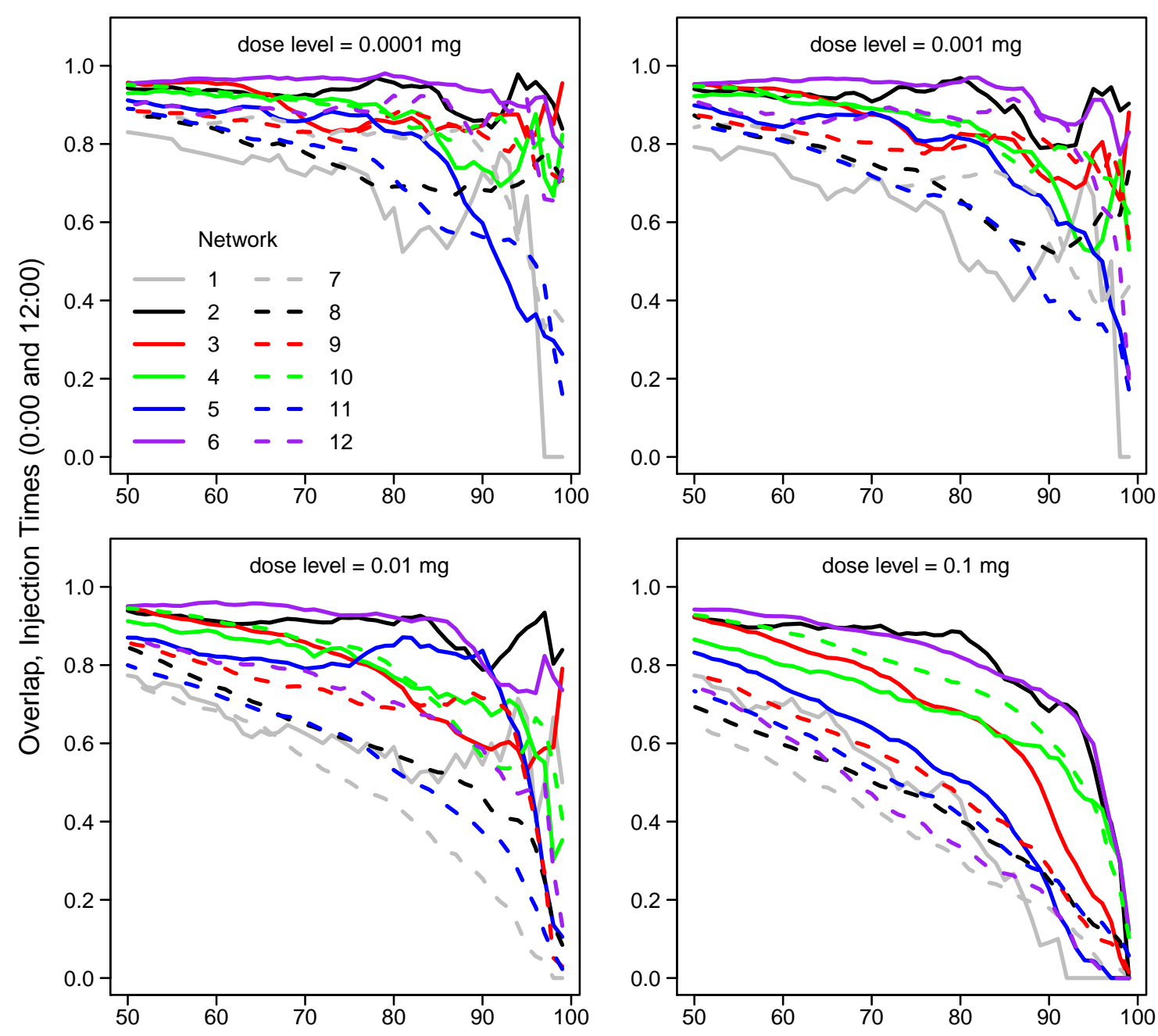

Percentile

Figure 5.29. Overlap of high percentile injection nodes for the networks for an injection time of 12:00 with those for an injection time of $0: 00$, for dose levels of $0.0001,0.001,0.01$, and 0.1 mg. 


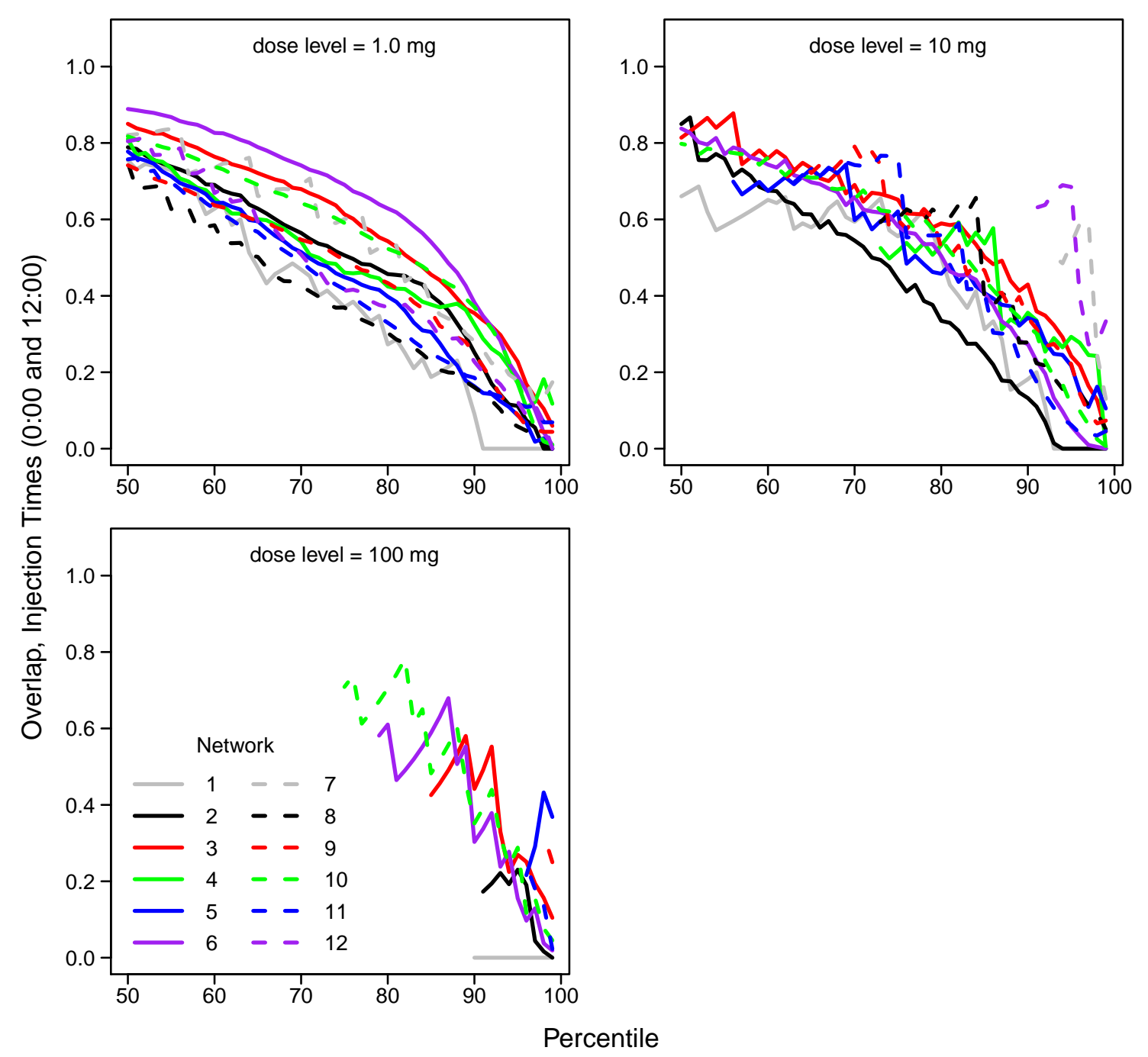

Figure 5.30. Overlap of high percentile injection nodes for the networks for an injection time of 12:00 with those for an injection time of $0: 00$, for dose levels of 1,10 , and $100 \mathrm{mg}$. 

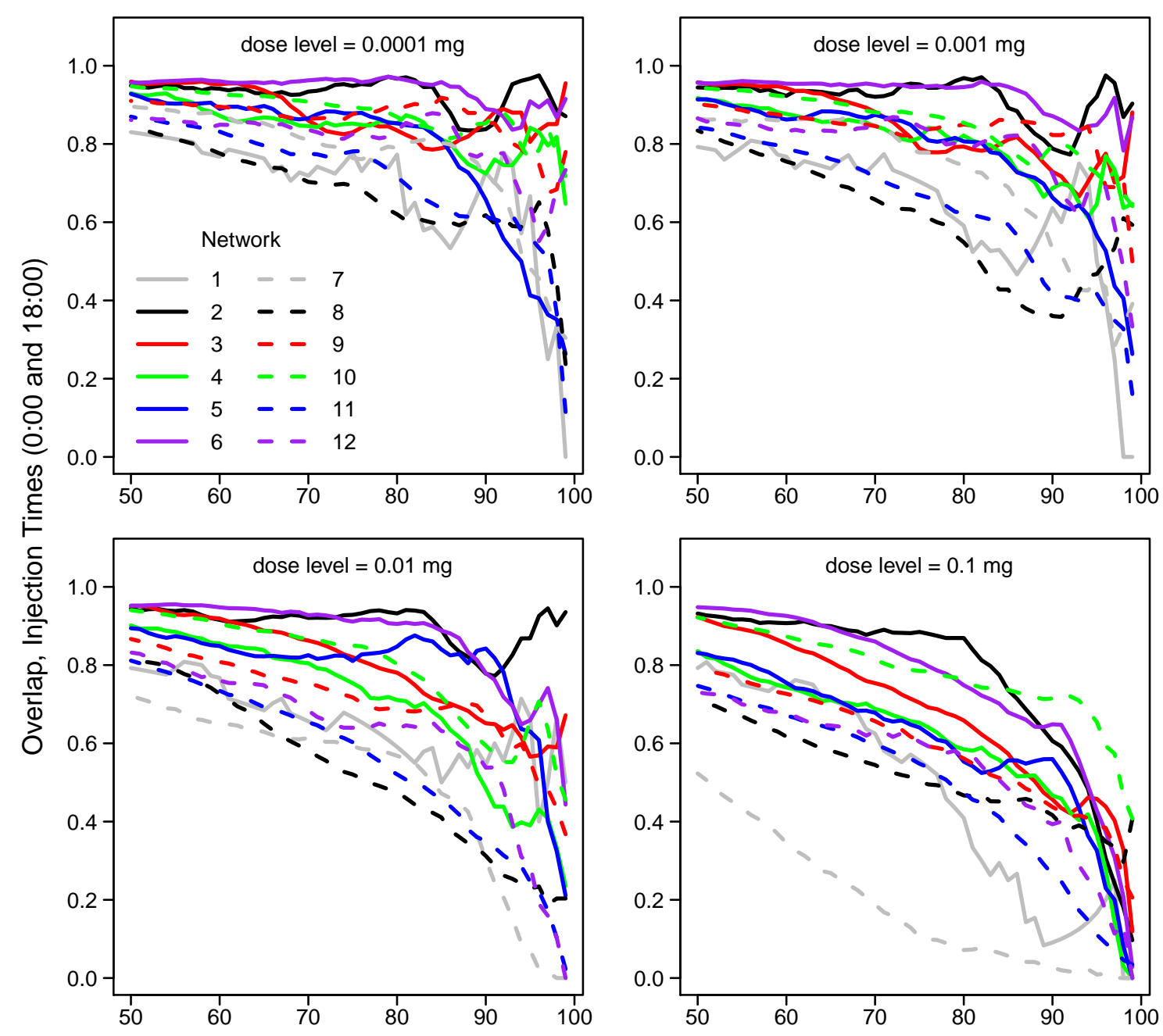

Percentile

Figure 5.31. Overlap of high percentile injection nodes for the networks for an injection time of 18:00 with those for an injection time of $0: 00$, for dose levels of $0.0001,0.001,0.01$, and 0.1 mg. 


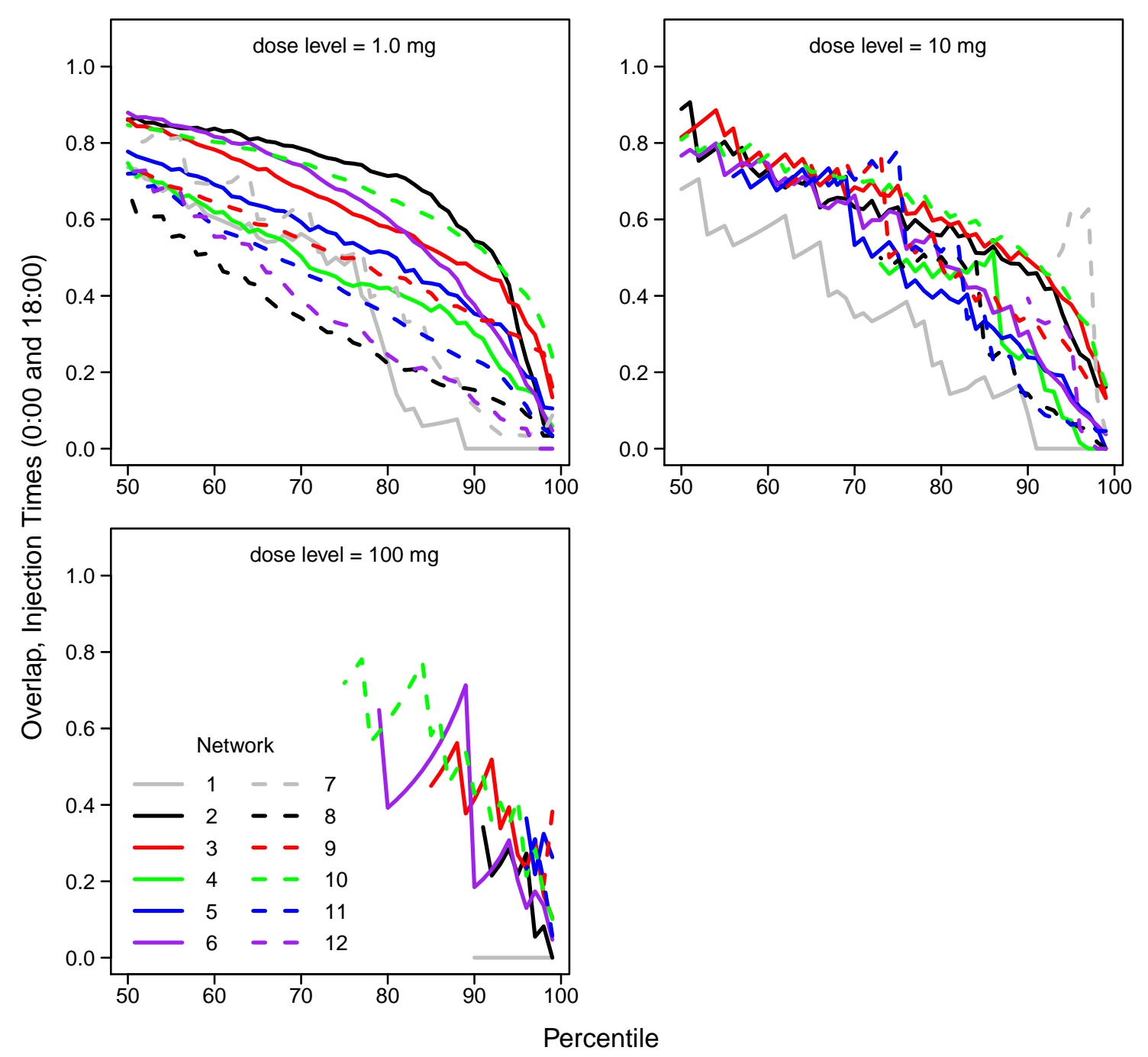

Figure 5.32. Overlap of high percentile injection nodes for the networks for an injection time of 18:00 with those for an injection time of $0: 00$, for dose levels of 1,10 , and $100 \mathrm{mg}$. 


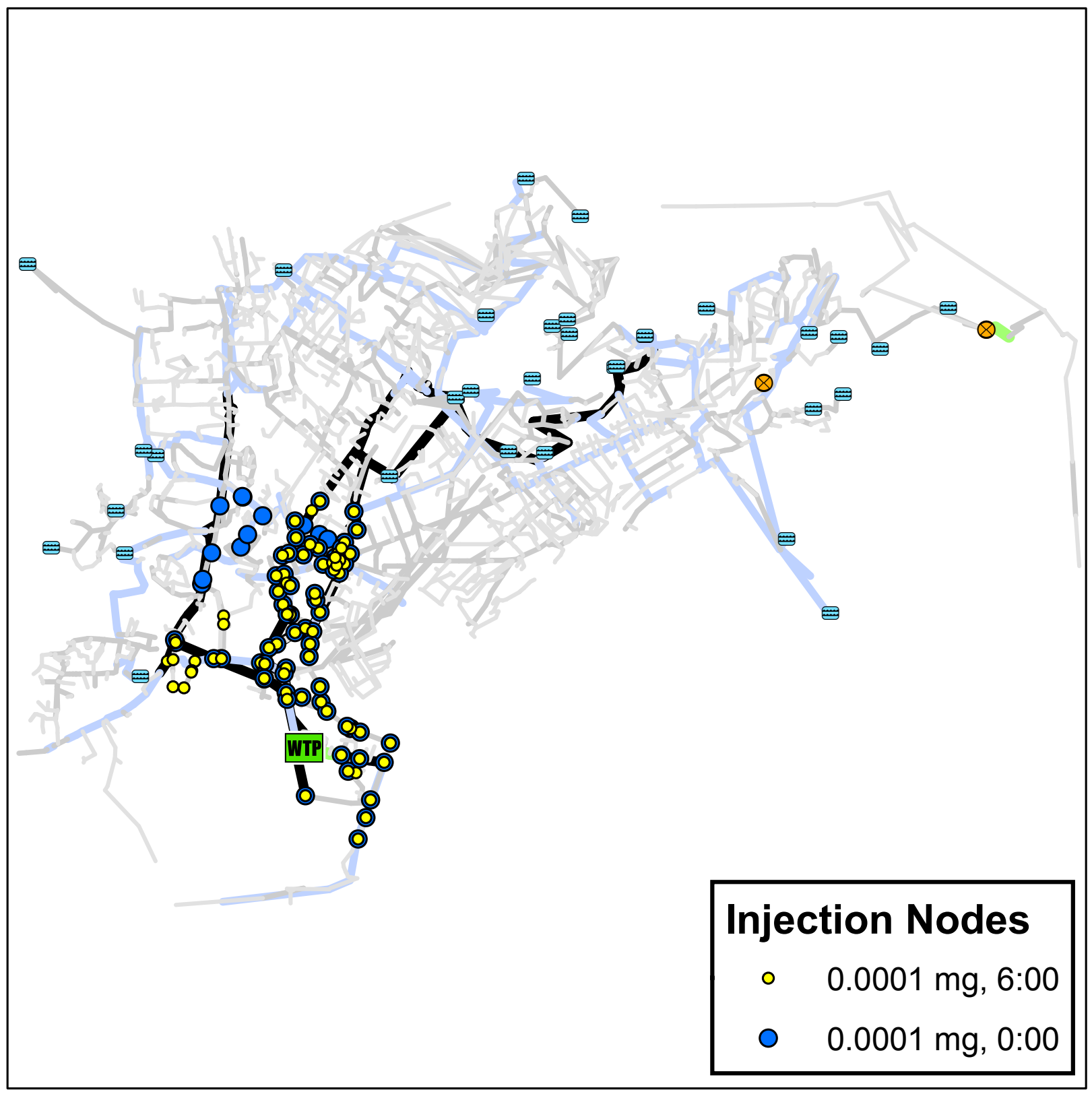

Figure 5.33. Network 4 showing locations of injection nodes associated with $95^{\text {th }}$ percentile or higher impacts at a dose level of $0.0001 \mathrm{mg}$ for injection times of 0:00 and 6:00. 


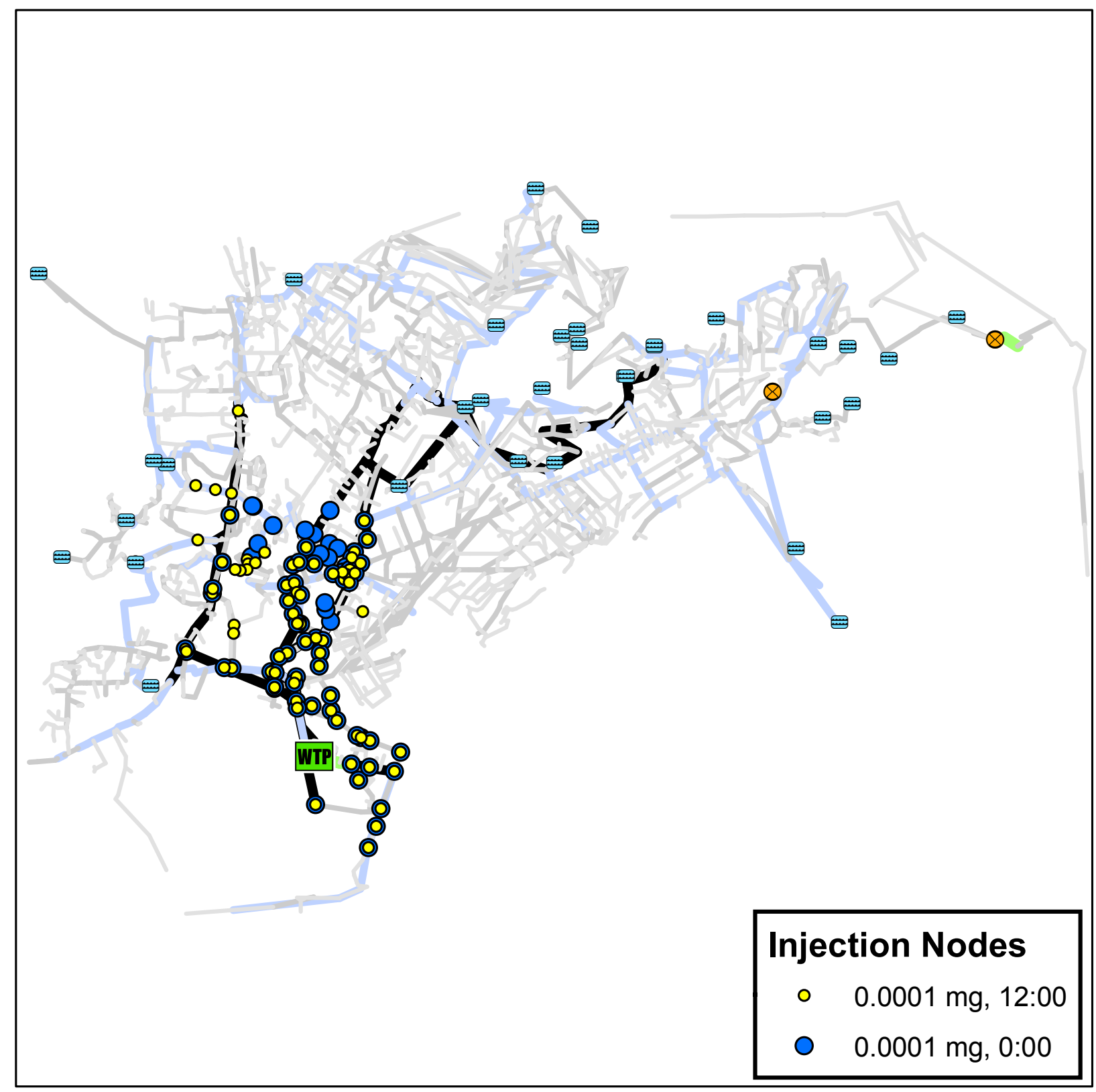

Figure 5.34. Network 4 showing locations of injection nodes associated with $95^{\text {th }}$ percentile or higher impacts at a dose level of $0.0001 \mathrm{mg}$ for injection times of 0:00 and 12:00. 


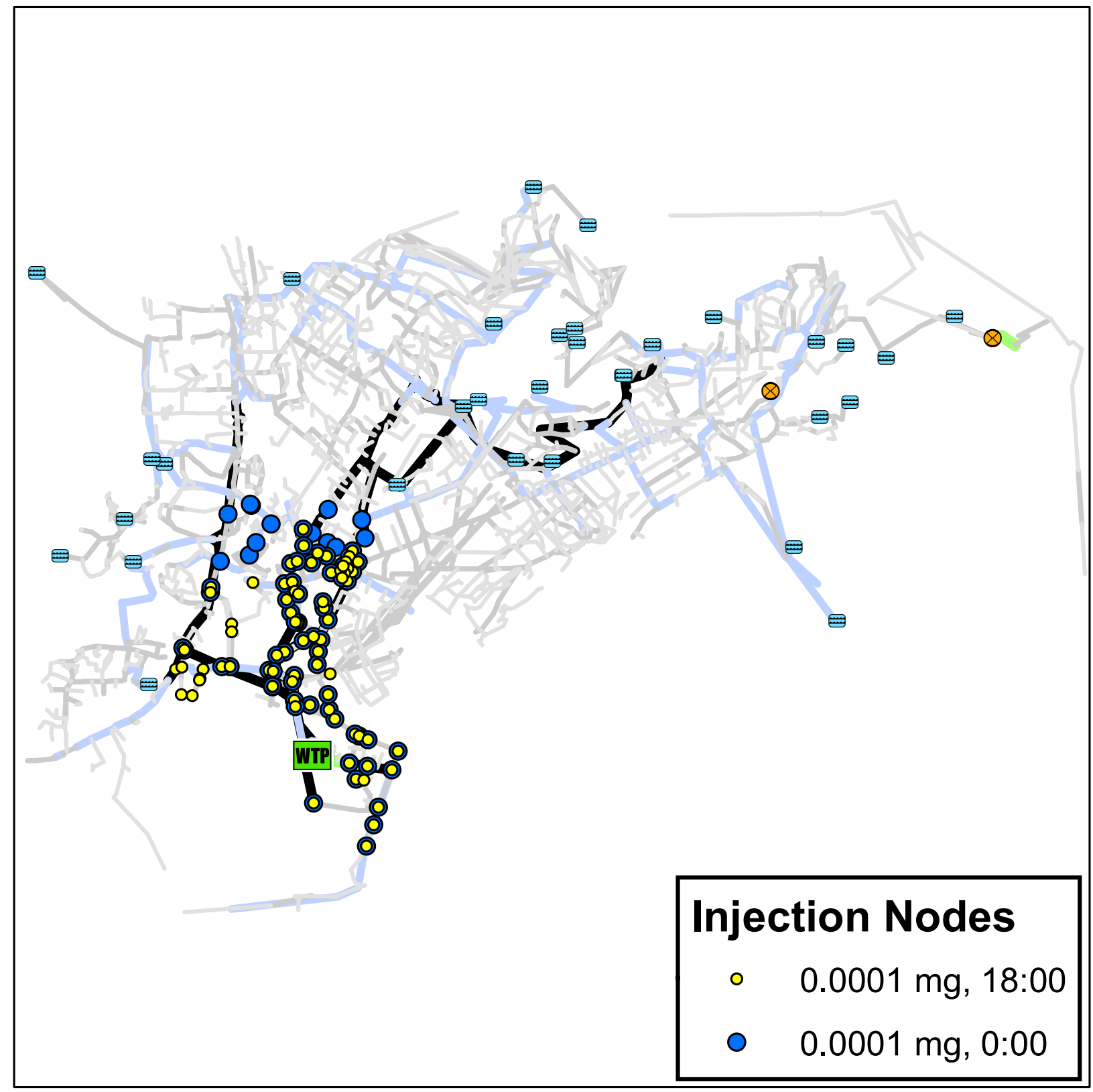

Figure 5.35. Network 4 showing locations of injection nodes associated with $95^{\text {th }}$ percentile or higher impacts at a dose level of $0.0001 \mathrm{mg}$ for injection times of 0:00 and 18:00. 


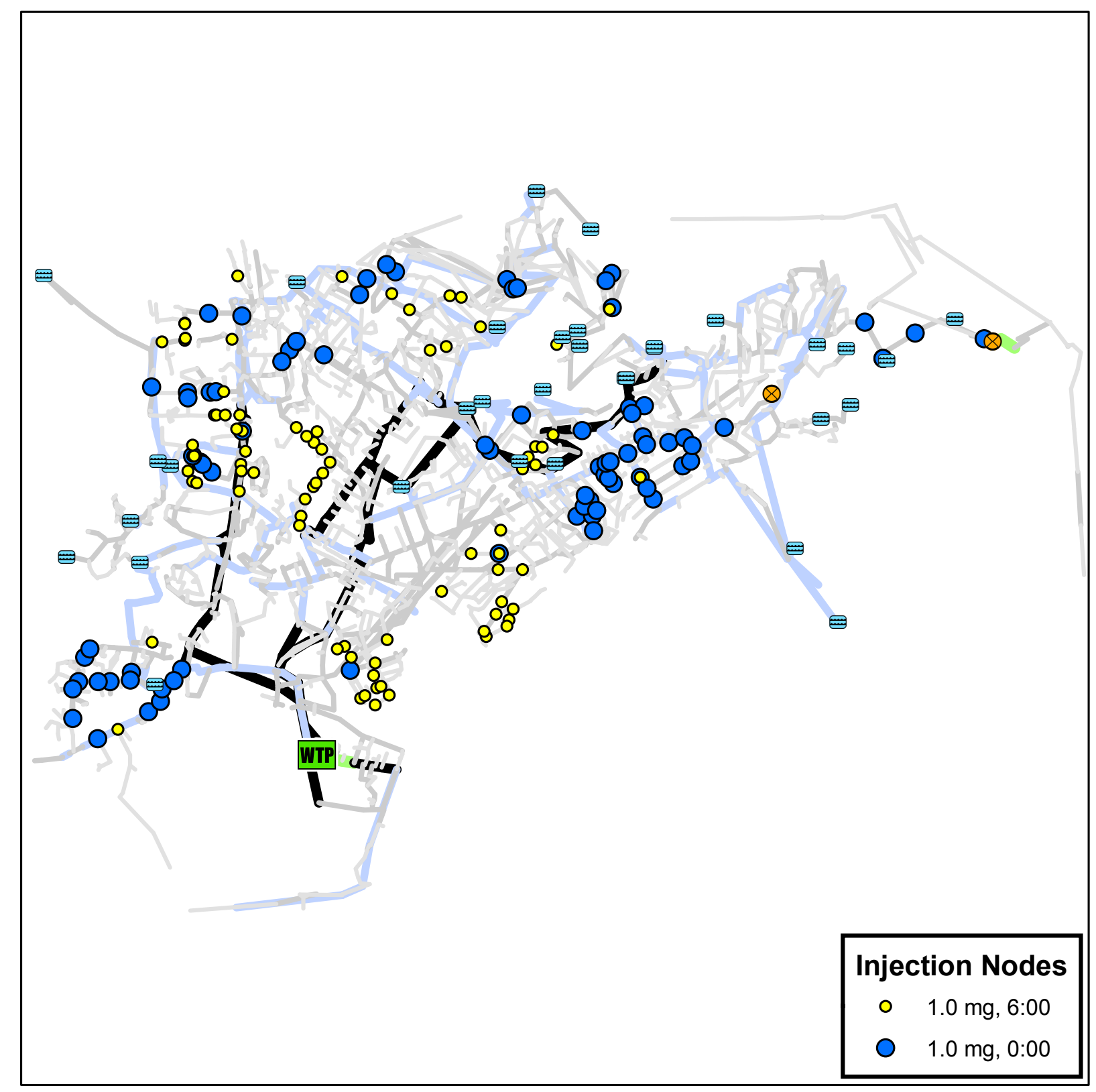

Figure 5.36. Network 4 showing locations of injection nodes associated with $95^{\text {th }}$ percentile or higher impacts at a dose level of $1.0 \mathrm{mg}$ for injection times of 0:00 and 6:00. 


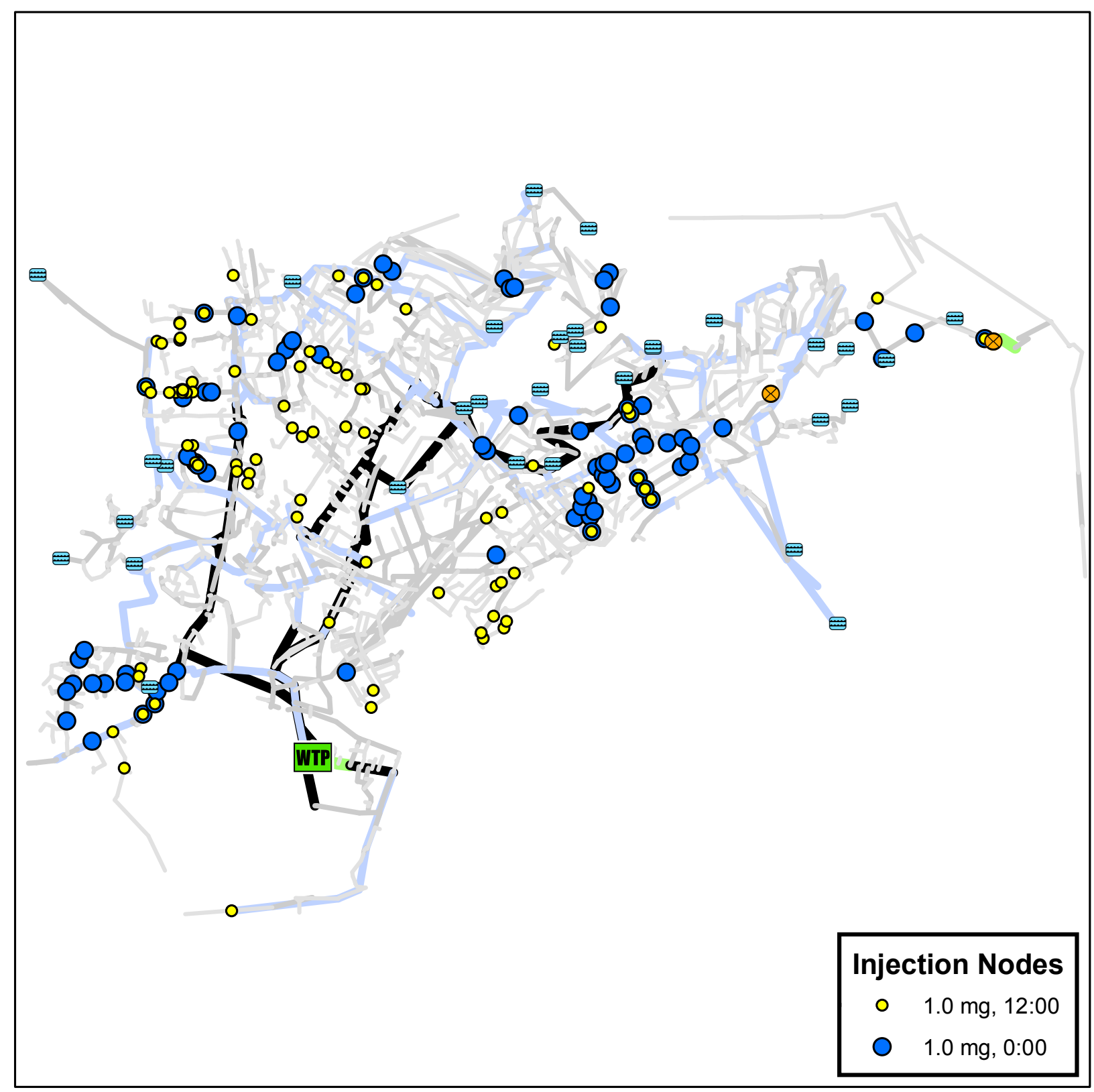

Figure 5.37. Network 4 showing locations of injection nodes associated with $95^{\text {th }}$ percentile or higher impacts at a dose level of $1.0 \mathrm{mg}$ for injection times of 0:00 and 12:00. 


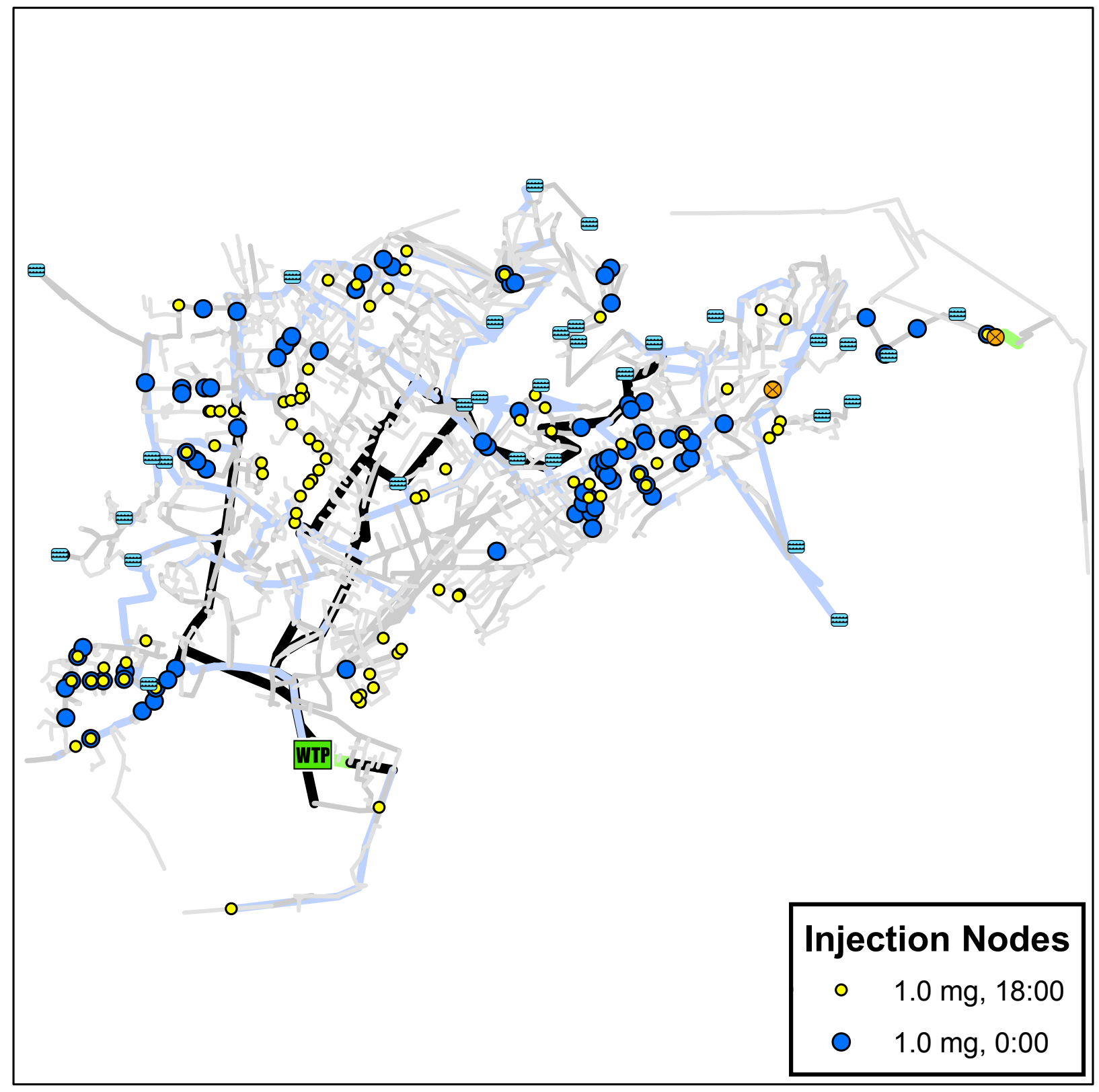

Figure 5.38. Network 4 showing locations of injection nodes associated with $95^{\text {th }}$ percentile or higher impacts at a dose level of $1.0 \mathrm{mg}$ for injection times of 0:00 and 18:00. 


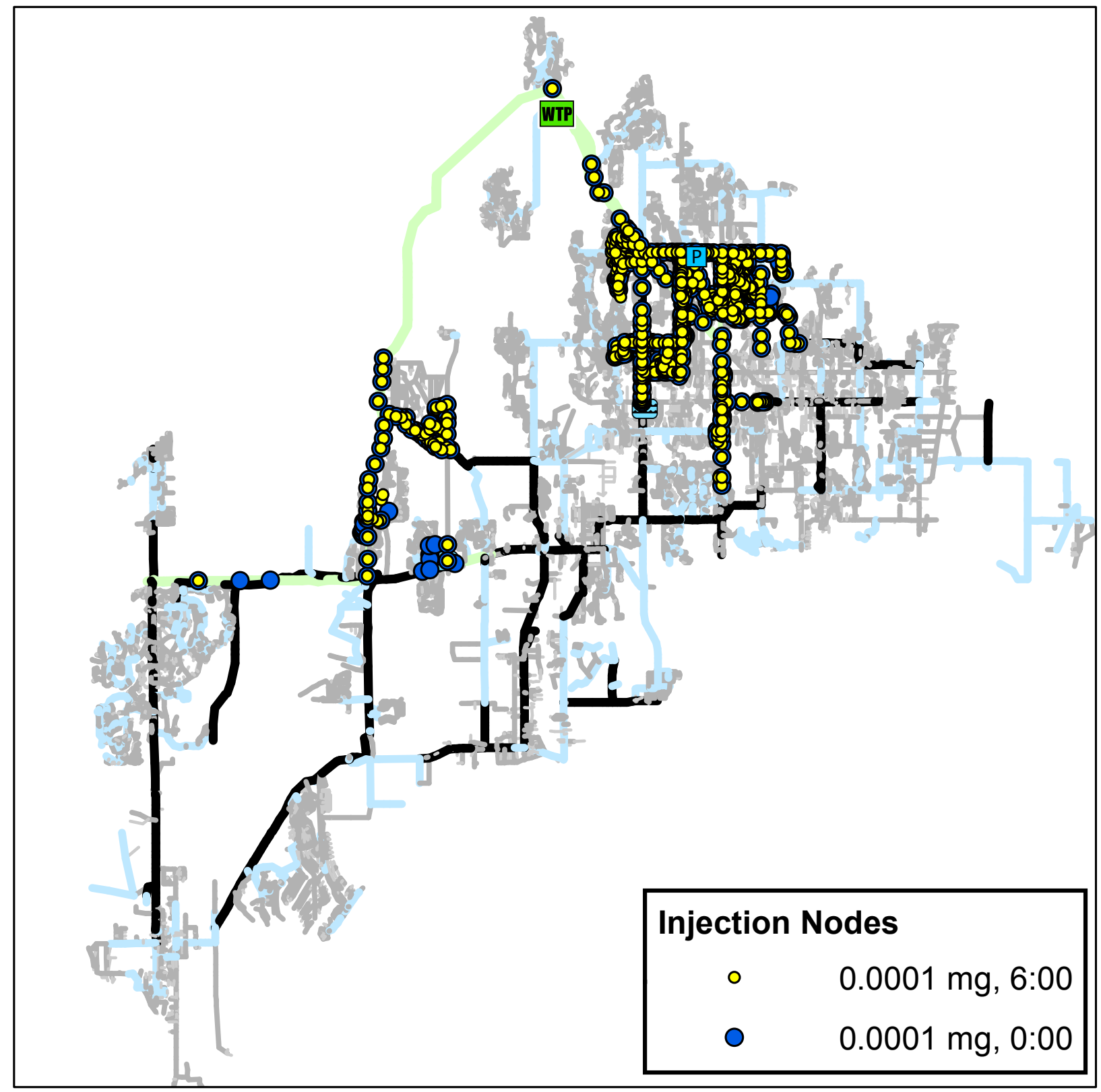

Figure 5.39. Network 6 showing locations of injection nodes associated with $95^{\text {th }}$ percentile or higher impacts at a dose level of $0.0001 \mathrm{mg}$ for injection times of 0:00 and 6:00. 


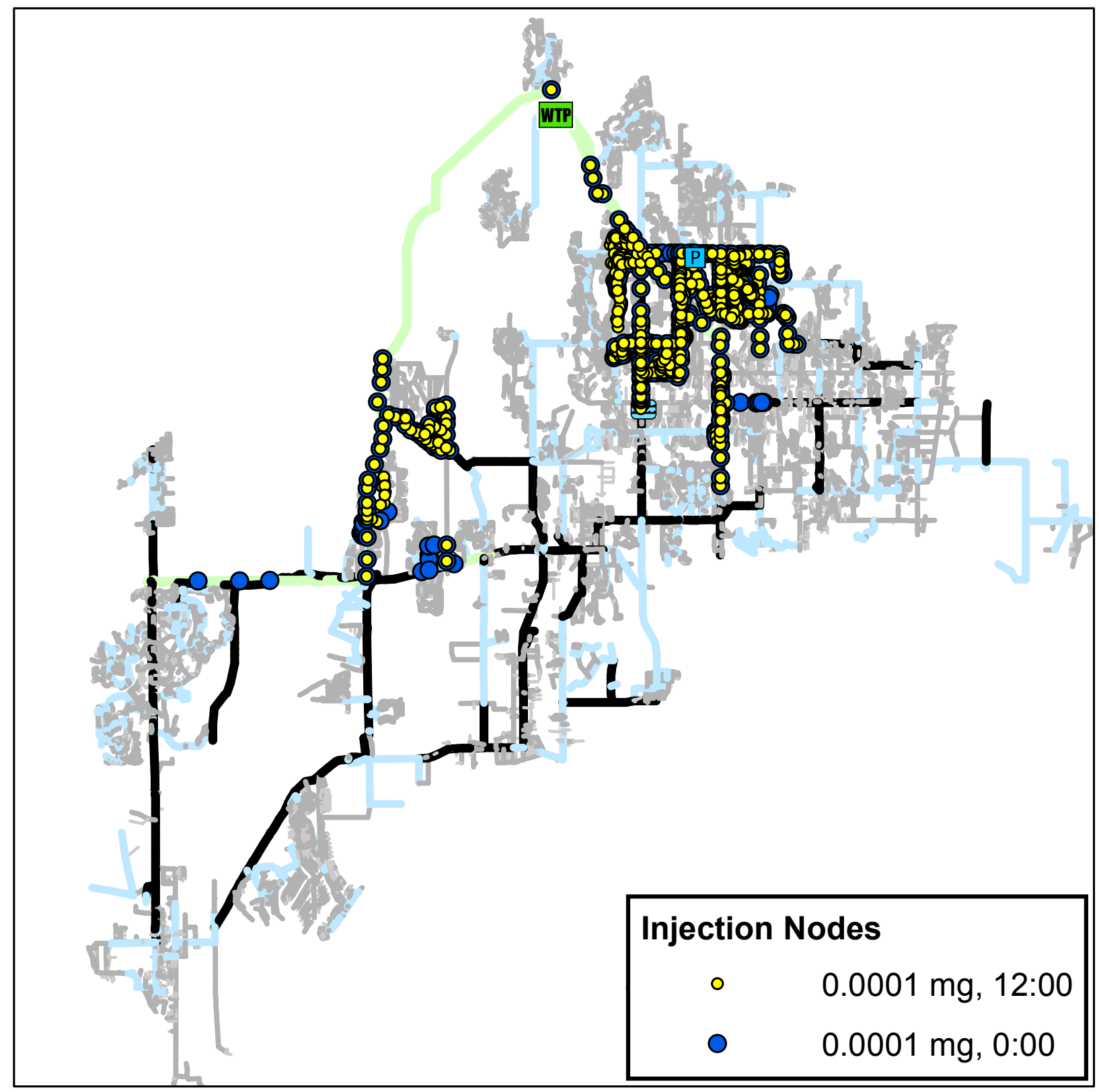

Figure 5.40. Network 6 showing locations of injection nodes associated with $95^{\text {th }}$ percentile or higher impacts at a dose level of $0.0001 \mathrm{mg}$ for injection times of 0:00 and 12:00. 


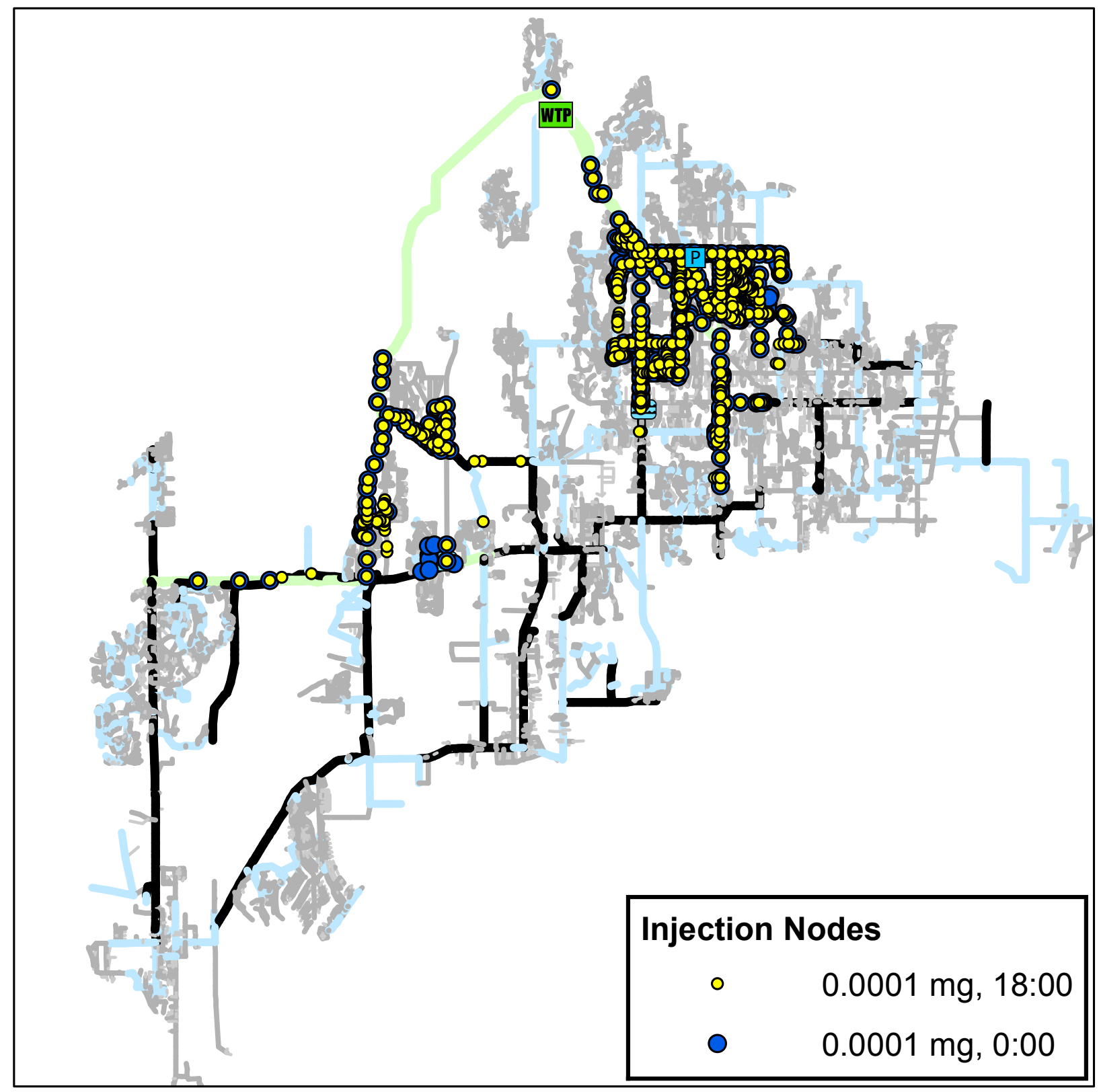

Figure 5.41. Network 6 showing locations of injection nodes associated with $95^{\text {th }}$ percentile or higher impacts at a dose level of $0.0001 \mathrm{mg}$ for injection times of 0:00 and 18:00. 


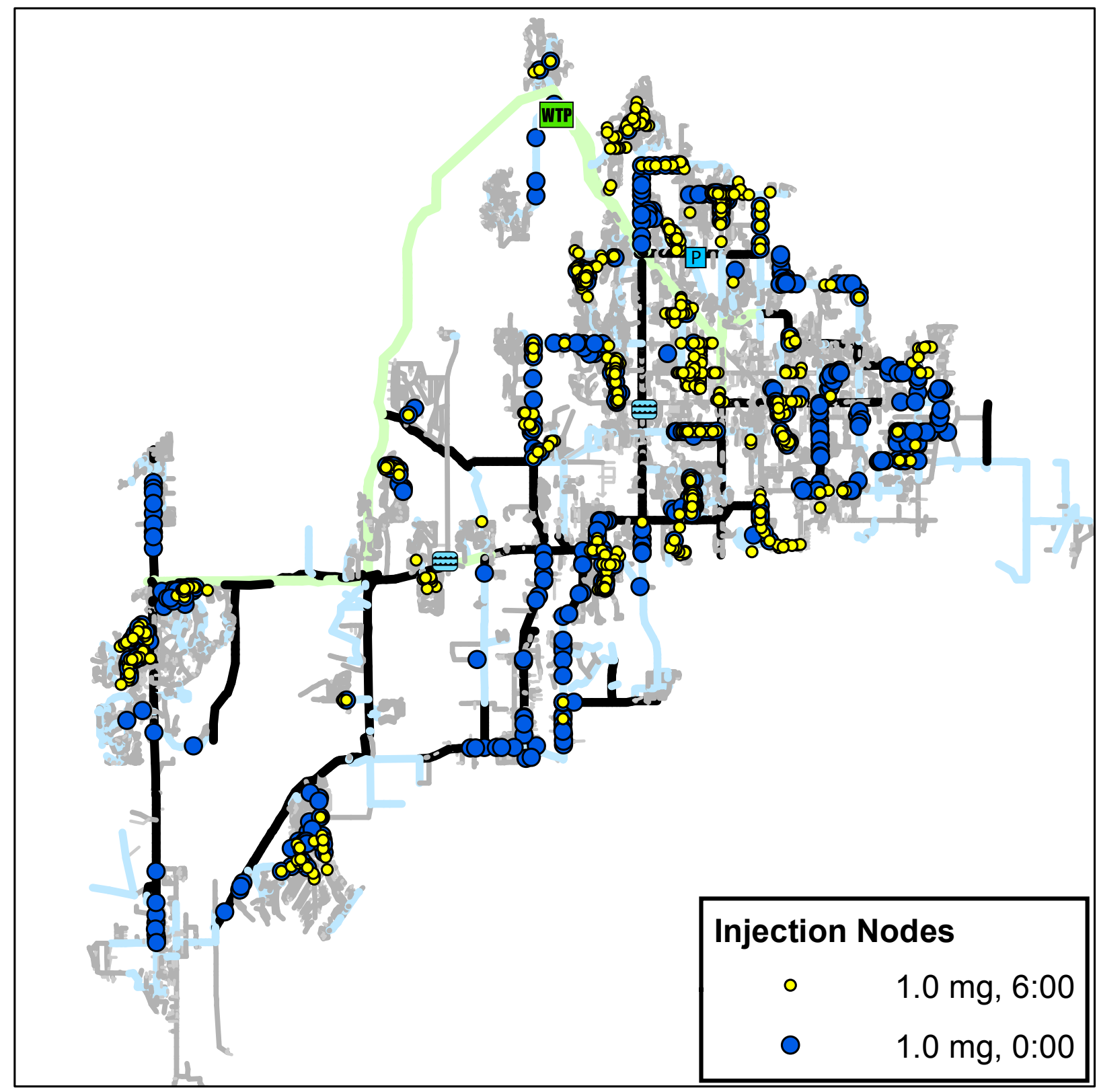

Figure 5.42. Network 6 showing locations of injection nodes associated with $95^{\text {th }}$ percentile or higher impacts at a dose level of $1.0 \mathrm{mg}$ for injection times of 0:00 and 6:00. 


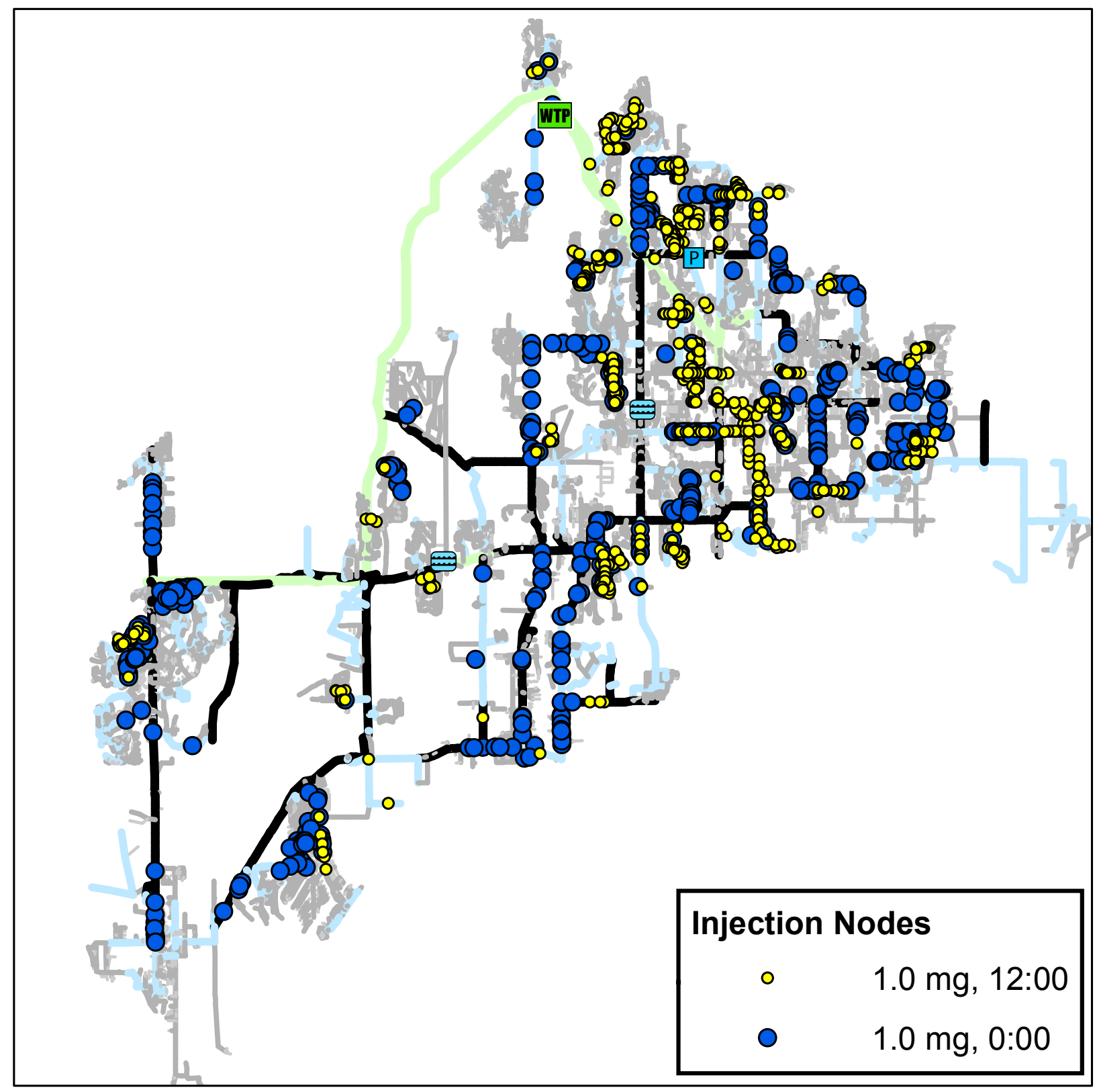

Figure 5.43. Network 6 showing locations of injection nodes associated with $95^{\text {th }}$ percentile or higher impacts at a dose level of $1.0 \mathrm{mg}$ for injection times of 0:00 and 12:00. 


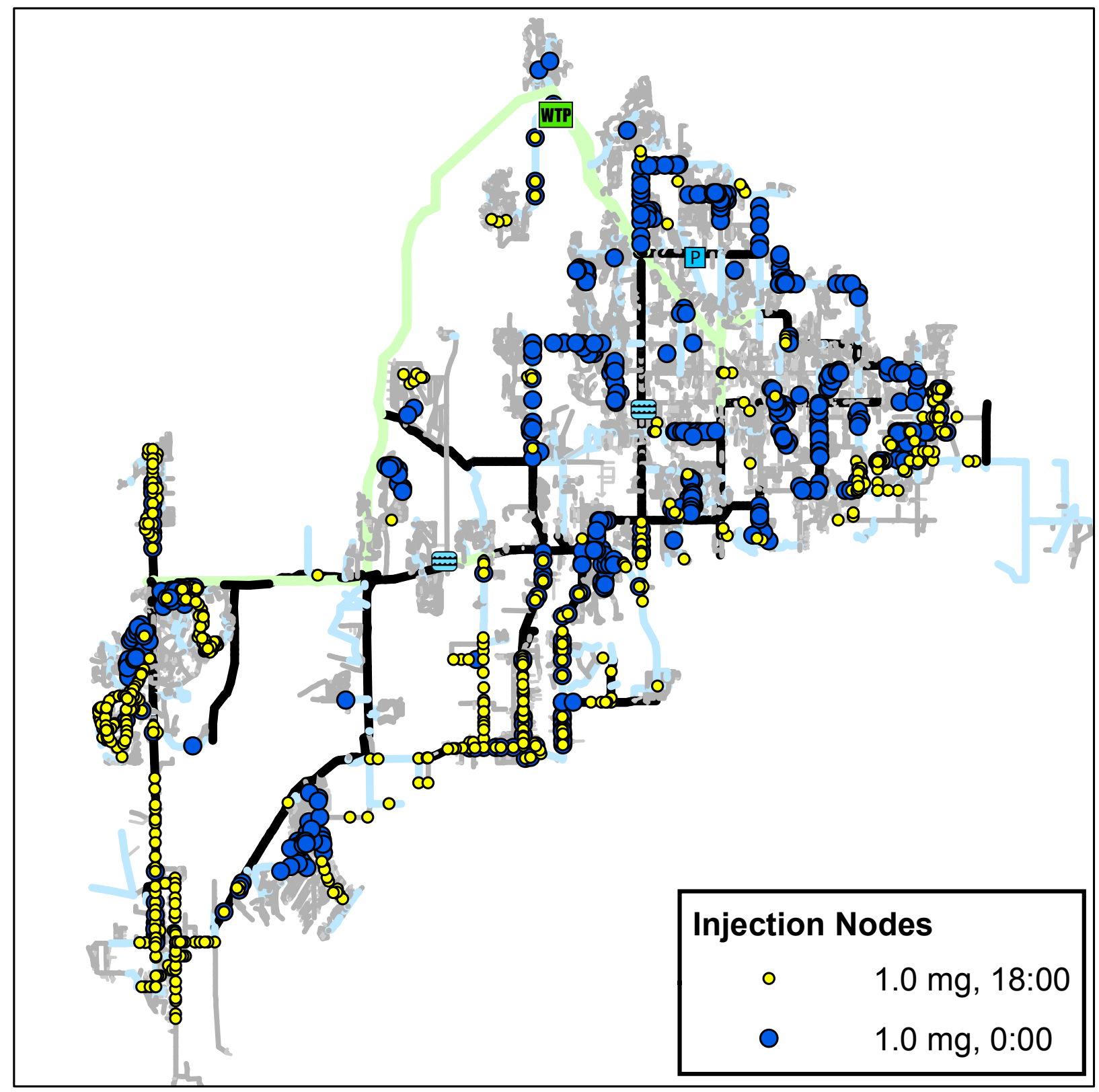

Figure 5.44. Network 6 showing locations of injection nodes associated with $95^{\text {th }}$ percentile or higher impacts at a dose level of $1.0 \mathrm{mg}$ for injection times of 0:00 and 18:00. 


\section{Section 6}

\section{Sensitivity of Impacts to Injection Mass}

To assess the sensitivity of impacts to injection mass, we consider two values for injection mass. The base case uses a realistic injection mass, which we designate $1 \mathrm{X}$. The second case uses ten times that mass and is labeled 10X. Figures 6.1 and 6.2 show that increasing injection mass by a factor of ten can have a significant influence on impacts for Network 12, except at the lowest dose levels. The pattern is similar for percentile impacts ranging from 50 to $100 \%$, except that as the percentile of the impact decreases below about $90 \%$, the range of dose levels over which the influence is small increases. For example, for the $90^{\text {th }}$ percentile, the impacts for the two different injection masses diverge above a dose level of about $0.0001 \mathrm{mg}$, while for the $50^{\text {th }}$ percentile, the impacts for the two cases diverge only for dose levels above about $0.001 \mathrm{mg}$. The impacts at the lowest dose levels do not increase as the injected mass increases because the number of people exposed does not increase as the injected mass increases. Doses will increase but the number of individuals with doses above the lowest dose levels does not; the contaminated area in the network does not continue to expand as more contaminant is injected.

Figures 6.3 and 6.4 provide the relative magnitudes of impacts for $1 \mathrm{X}$ and 10X injection masses versus dose level for all the networks. The effect of increasing injection mass is qualitatively similar for all the networks considered. Relative impacts tend to increase as the dose level increases, but with considerable quantitative variability among the networks. Overall behavior across the networks becomes more consistent for percentiles below about $90 \%$. However, there is still considerable variability from network to network in their relative response to increased injection mass. For example, for $60^{\text {th }}$ percentile impacts and a dose level of $0.1 \mathrm{mg}$, the relative increase in impact as the injection mass is increased by a factor of ten is about two for Network 6 and about 15 for Network 7. (For comparison, absolute values for impacts for the networks for 1X injections are provided in Figures 3.1 to 3.3.)

The relative influence of changes in injection mass on impact for the different networks is shown quantitatively in Figures 6.5 through 6.9. The plots illustrate how varying injection mass over five orders of magnitude affects impacts relative to the impact for the baseline case of a $1 \mathrm{X}$ mass injection, for dose levels ranging from $0.0001 \mathrm{mg}$ to $1.0 \mathrm{mg}$. Results are provided for four impact levels (percentiles equal to 100, 95, 75, and 50). At the lower dose levels, impact can be quite insensitive to injection mass over a considerable range in injection mass, but sensitivity varies considerably among the networks. For example, for a dose level of $0.0001 \mathrm{mg}$ (Figure 6.5), the $50^{\text {th }}$ percentile impact for Network 6 shows minimal change as injection mass is varied from about $0.01 \mathrm{X}$ to $10 \mathrm{X}$, a change in injected mass of three orders of magnitude. However, for Network 11 
the change is about 10, substantially larger, but still small relative to the change in injection mass. At higher dose levels, impacts increase considerably as injection mass increases, but again with large variability among the networks.

Using violin plots, the distribution of impacts associated with the $1 \mathrm{X}$ and $10 \mathrm{X}$ injection masses is provided in Figures 6.10 and 6.11 for Network 12. Consistent with the results in Figures 6.1 and 6.2, the distributions for the two cases can be considerably different, except at the lowest dose level.

The scatter plots in Figures 6.12 and 6.13 show for Network 12 that on a node-by-node basis the impacts associated with the $10 \mathrm{X}$ injection mass are usually larger than those associated with the $1 \mathrm{X}$ injection mass, but, in general, there is no linear relationship between the impacts for the two cases. Impacts are often much larger for the 10X case, as would be expected. However, the overlap of high percentile nodes for the two cases can be small at and above the $80^{\text {th }}$ percentile impact level for dose levels of $0.1 \mathrm{mg}$ and higher (Figures 6.14 and 6.15). Increasing the injection mass can result in a substantial shifting of the locations of the high-percentile injection nodes. Figure 6.16 shows the low overlap of the highest percentile nodes for Network 12, particularly for the higher dose levels.

Considering all the networks, Figures 6.17 and 6.18 shows the overlap of injection nodes for the two injection masses. The overlap is high for a dose level of $0.0001 \mathrm{mg}$ for most of the networks for all levels of impact. However, as the dose level increases, the degree of overlap decreases considerably, although there is considerable variability across the networks.

The locations of injection nodes in Network 4 associated with impacts at and above the $95^{\text {th }}$ percentile for injection masses of $1 \mathrm{X}$ and 10X are shown in Figure 6.19 for a dose level of $0.0001 \mathrm{mg}$. The overlap of the nodes is high. Figure 6.20 compares the location of $95^{\text {th }}$ percentile and higher injection nodes for Network 4 for injection masses of $1 \mathrm{X}$ and $10 \mathrm{X}$ for a dose level of $1.0 \mathrm{mg}$. In this case, the overlap is low. Figures 6.21 and 6.22 provide a similar comparison for Network 6 and demonstrate similar results, all of which are consistent with the overlap plots shown in Figures 6.17 and 6.18 .

Impacts can show considerable sensitivity to large increases in injected mass, both in terms of ranked impacts and overlap of high percentile nodes. However, at low dose levels the sensitivity even to large increases in injected mass is generally small. 


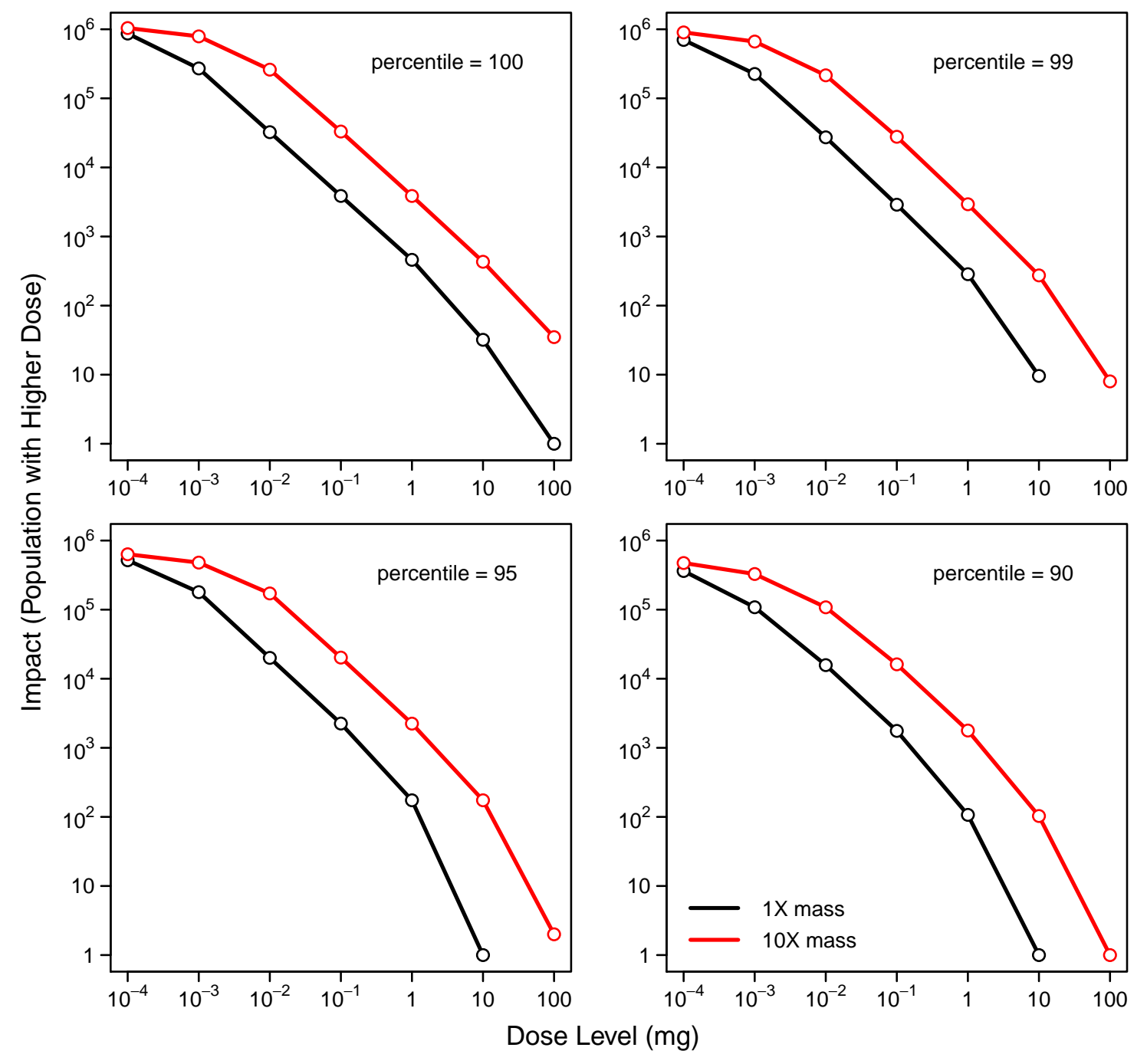

Figure 6.1. Impacts associated with injection masses of $1 \mathrm{X}$ and $10 \mathrm{X}$ for Network 12 as a function of dose level, for the $90^{\text {th }}, 95^{\text {th }}, 99^{\text {th }}$, and $100^{\text {th }}$ percentile injection nodes. 


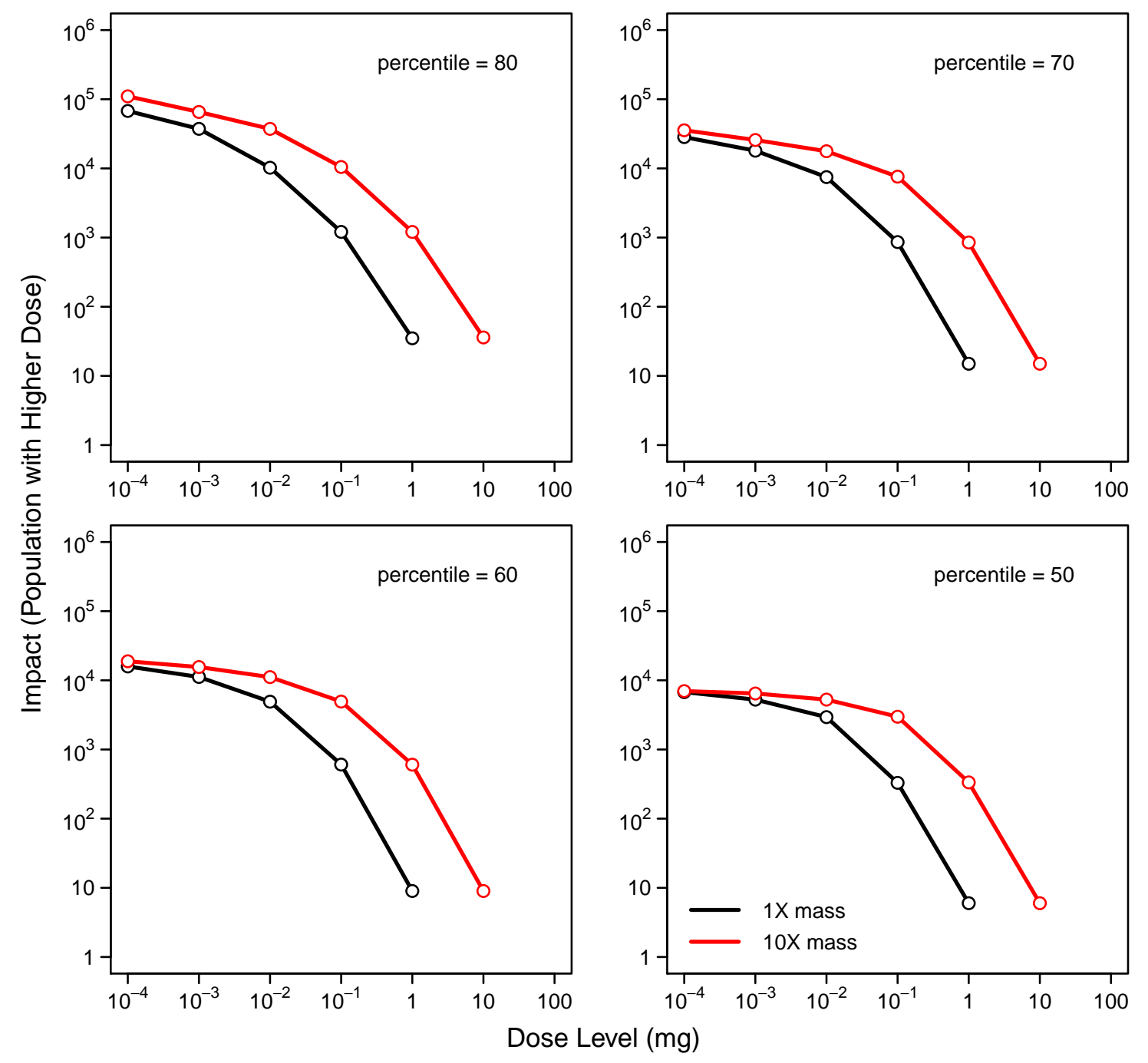

Figure 6.2. Impacts associated with injection masses of $1 \mathrm{X}$ and $10 \mathrm{X}$ for Network 12 as a function of dose level, for the $50^{\text {th }}, 60^{\text {th }}, 70^{\text {th }}$, and $80^{\text {th }}$ percentile injection nodes. 


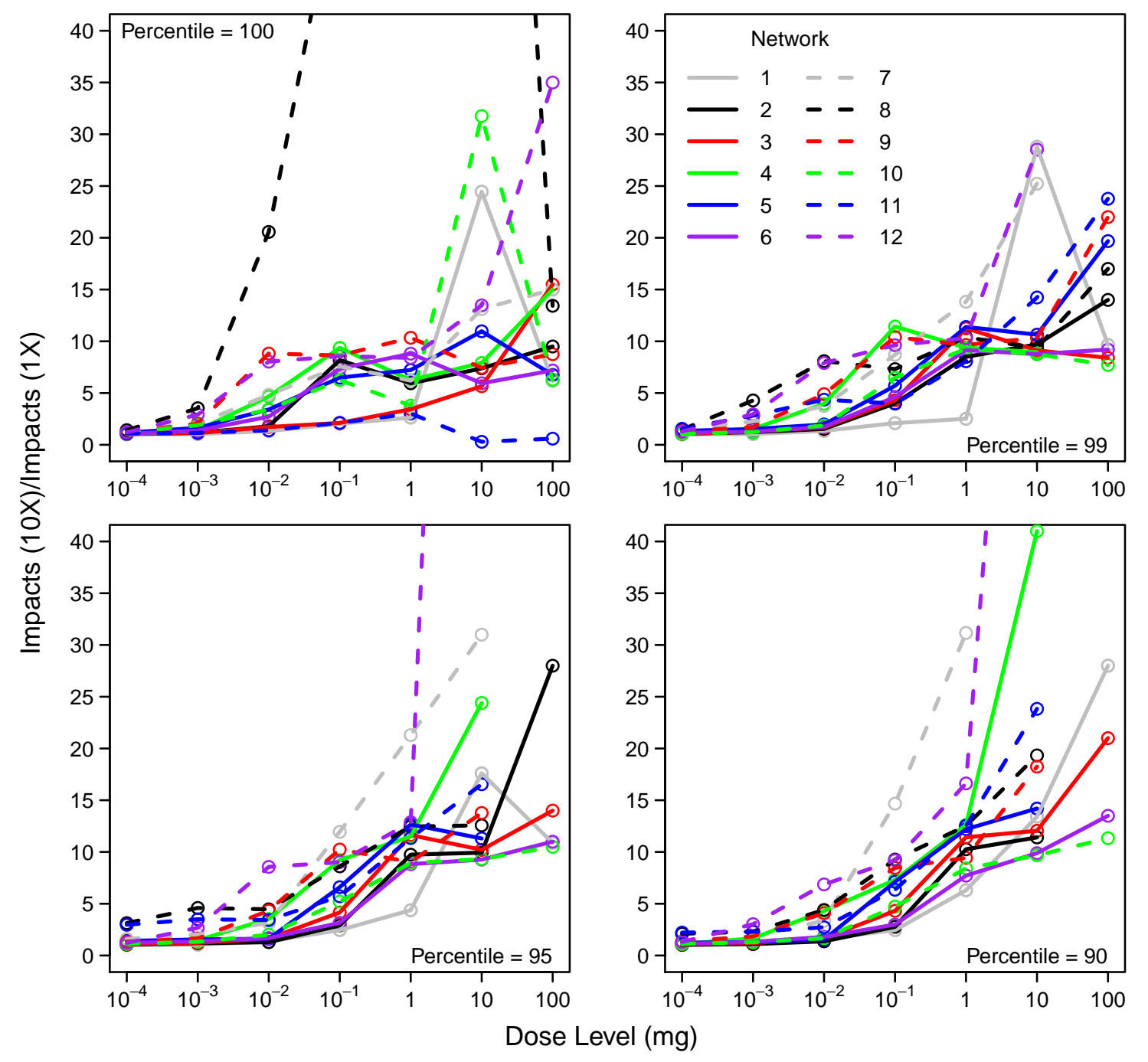

Figure 6.3. Impact for a $10 \mathrm{X}$ injection mass relative to the impact for a $1 \mathrm{X}$ injection mass, as a function of dose level, for the $90^{\text {th }}, 95^{\text {th }}, 99^{\text {th }}$, and $100^{\text {th }}$ percentile injection nodes. 


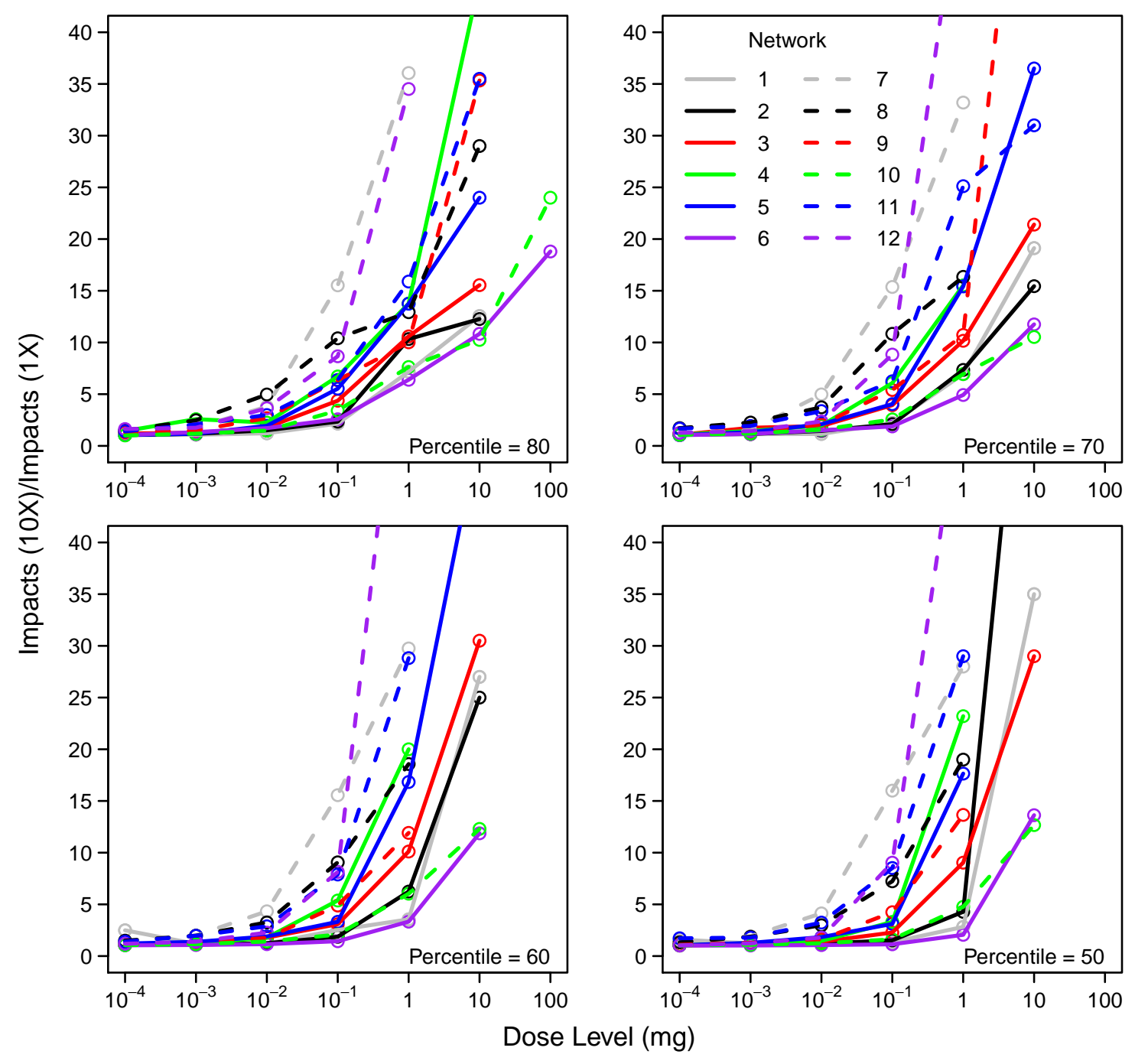

Figure 6.4. Impact for a $10 \mathrm{X}$ injection mass relative to the impact for a $1 \mathrm{X}$ injection mass, as a function of dose level, for the $50^{\text {th }}, 60^{\text {th }}, 70^{\text {th }}$, and $80^{\text {th }}$ percentile injection nodes. 


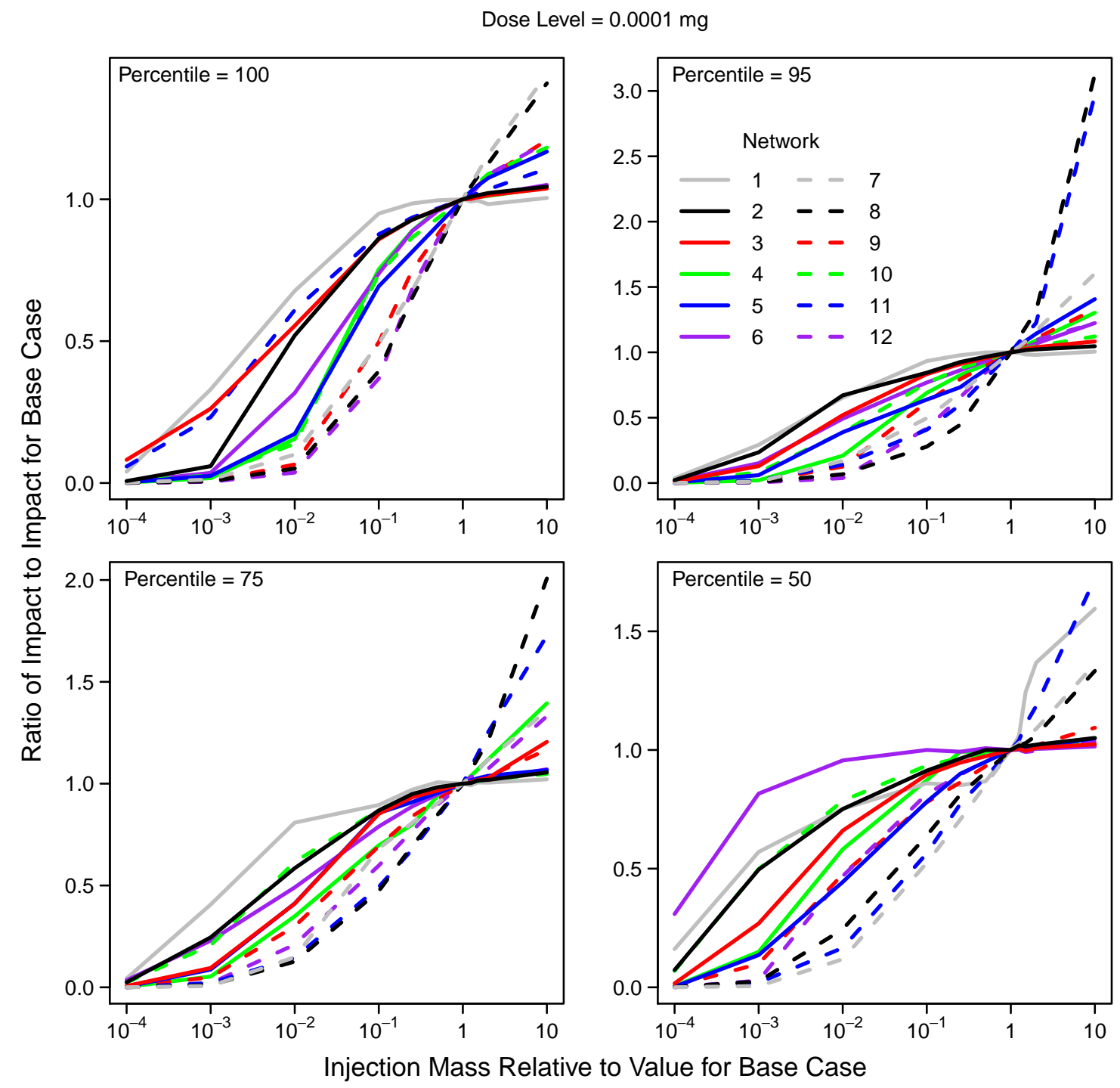

Figure 6.5. Variation in impacts as a function of injection mass for a dose level of $0.0001 \mathrm{mg}$, for the $100^{\text {th }}, 95^{\text {th }}, 75^{\text {th }}$, and $50^{\text {th }}$ percentile injection nodes. All impacts are relative to those for an injection mass of $1 X$. 


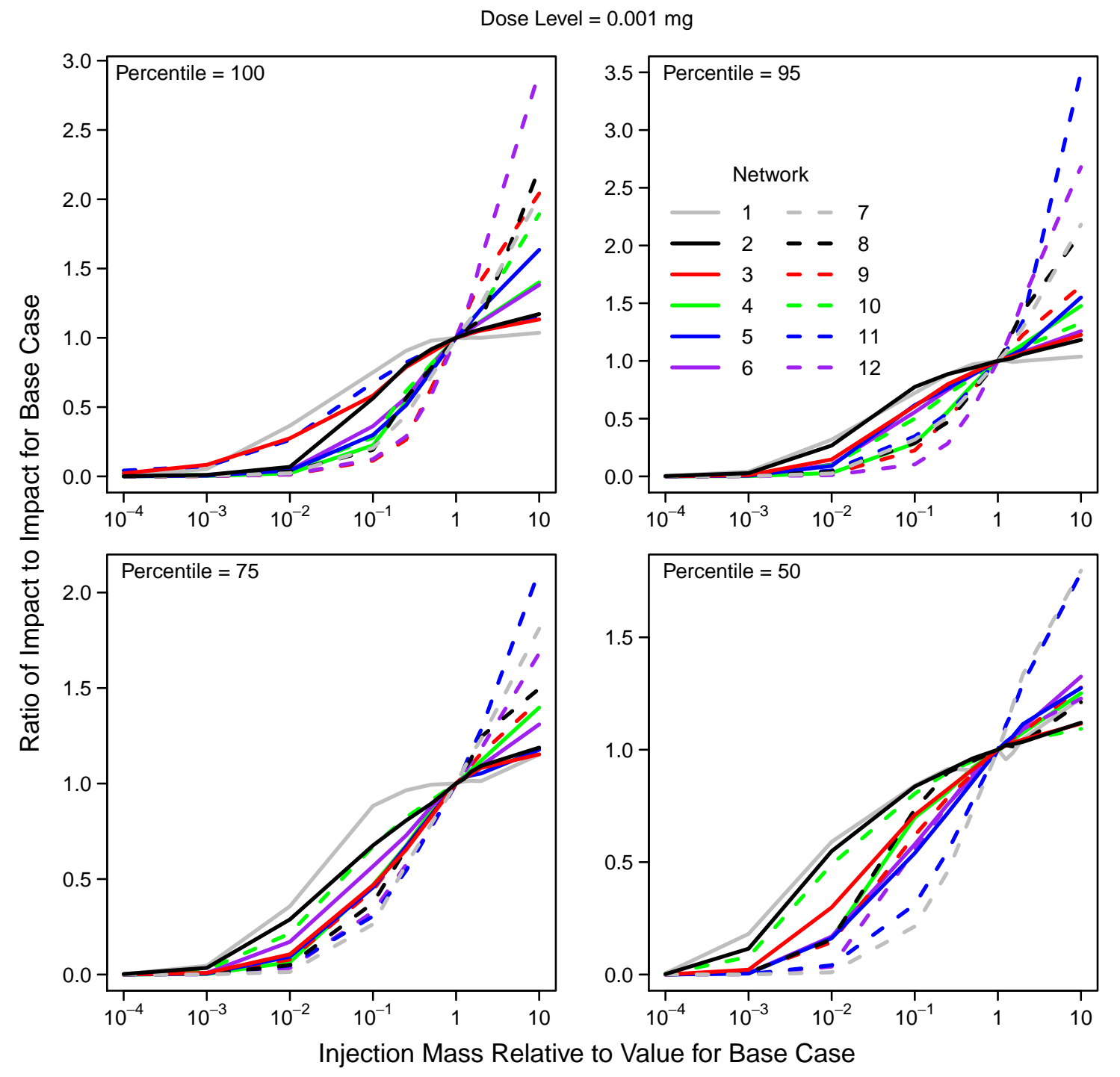

Figure 6.6. Variation in impacts as a function of injection mass for a dose level of $0.001 \mathrm{mg}$, for the $100^{\text {th }}, 95^{\text {th }}, 75^{\text {th }}$, and $50^{\text {th }}$ percentile injection nodes. All impacts are relative to those for an injection mass of $1 \mathrm{X}$. 


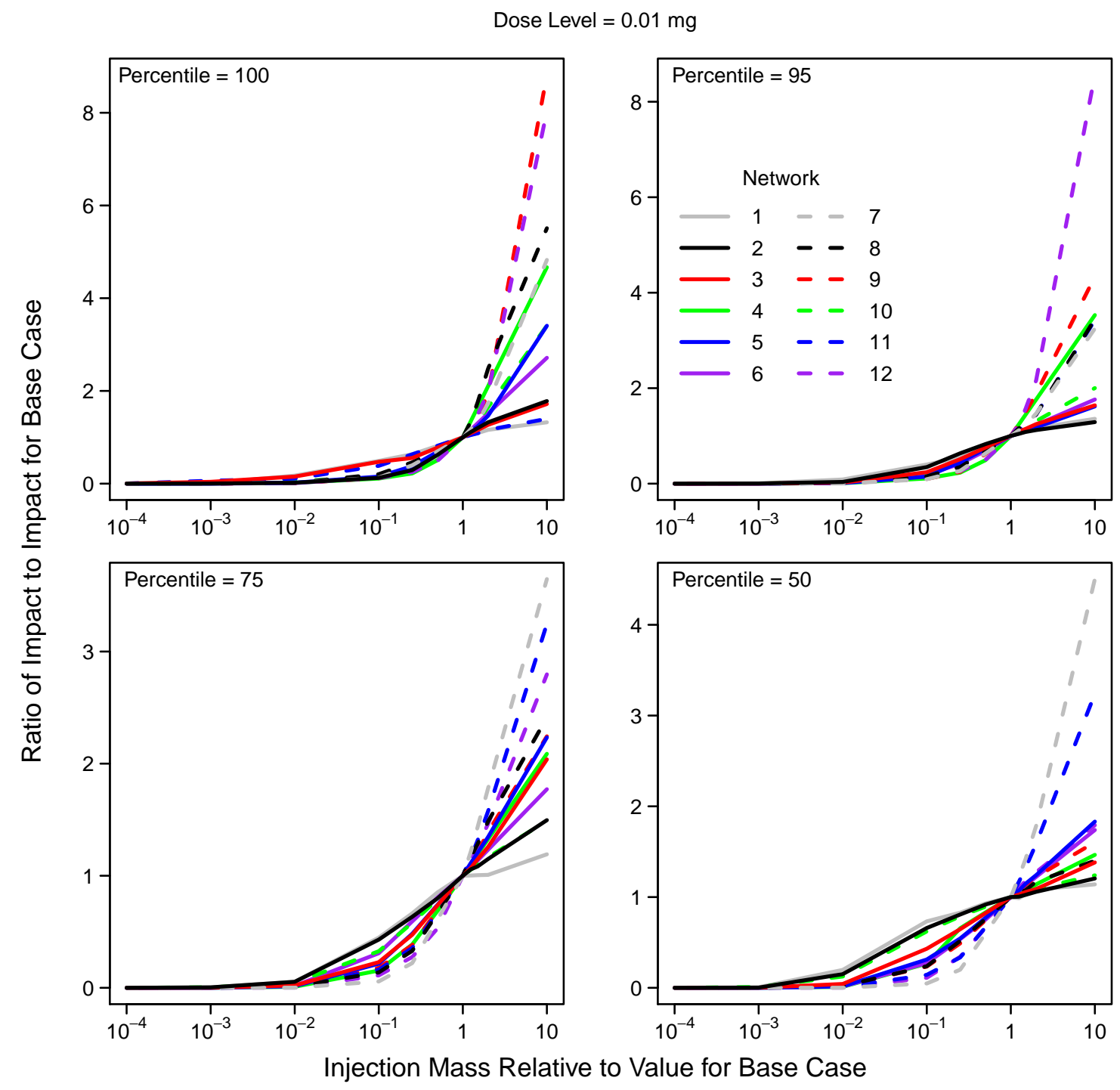

Figure 6.7. Variation in impacts as a function of injection mass for a dose level of $0.01 \mathrm{mg}$, for the $100^{\text {th }}, 95^{\text {th }}, 75^{\text {th }}$, and $50^{\text {th }}$ percentile injection nodes. All impacts are relative to those for an injection mass of $1 X$. 


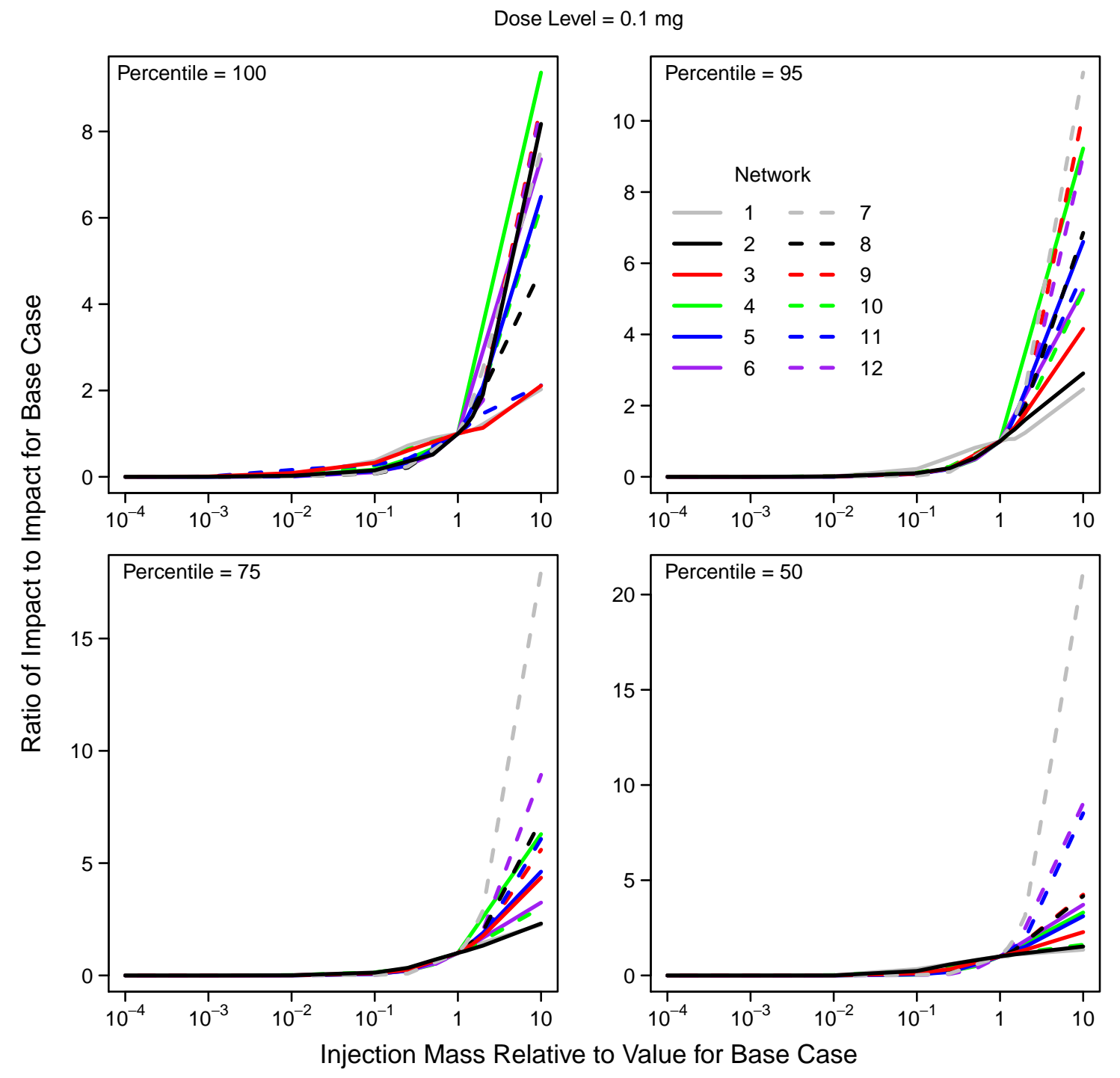

Figure 6.8. Variation in impacts as a function of injection mass for a dose level of $0.1 \mathrm{mg}$, for the $100^{\text {th }}, 95^{\text {th }}, 75^{\text {th }}$, and $50^{\text {th }}$ percentile injection nodes. All impacts are relative to those for an injection mass of $1 X$. 


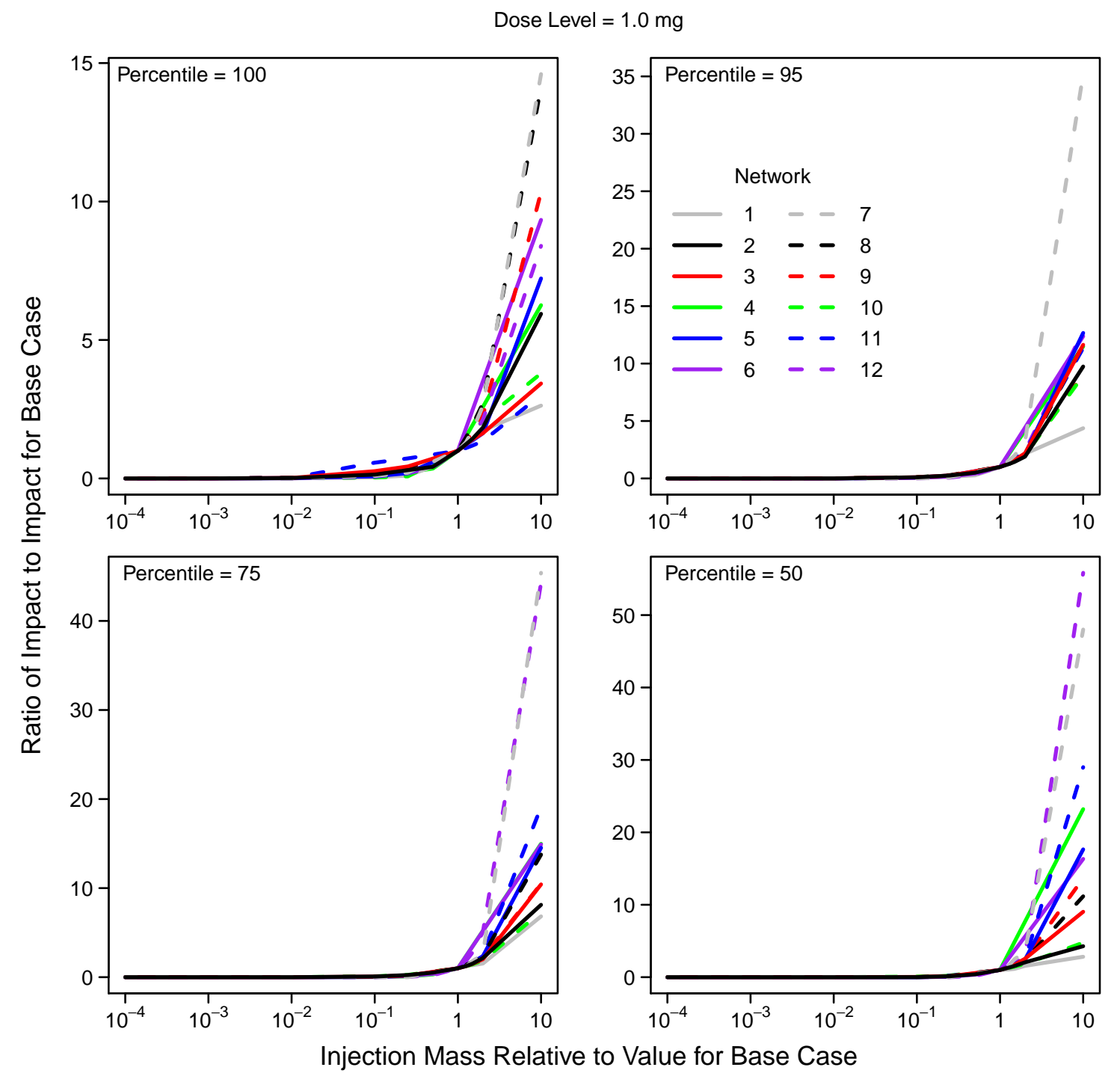

Figure 6.9. Variation in impacts as a function of injection mass for a dose level of $1 \mathrm{mg}$, for the $100^{\text {th }}, 95^{\text {th }}, 75^{\text {th }}$, and $50^{\text {th }}$ percentile injection nodes. All impacts are relative to those for an injection mass of $1 \mathrm{X}$. 


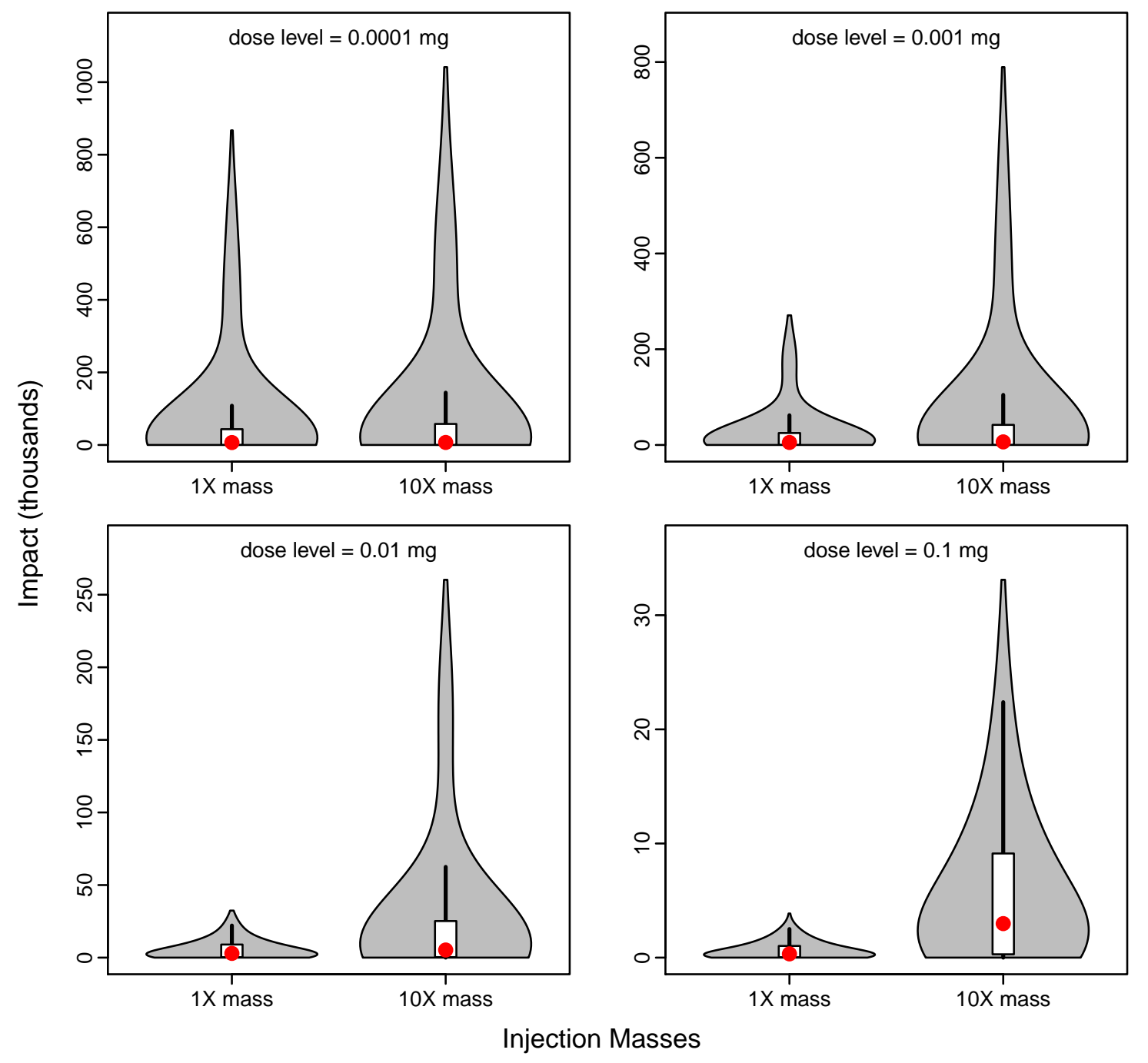

Figure 6.10. Distributions of impacts for Network 12 across all injection nodes for injection masses of $1 \mathrm{X}$ and 10X, for dose levels of $0.0001,0.001,0.01$, and $0.1 \mathrm{mg}$. 


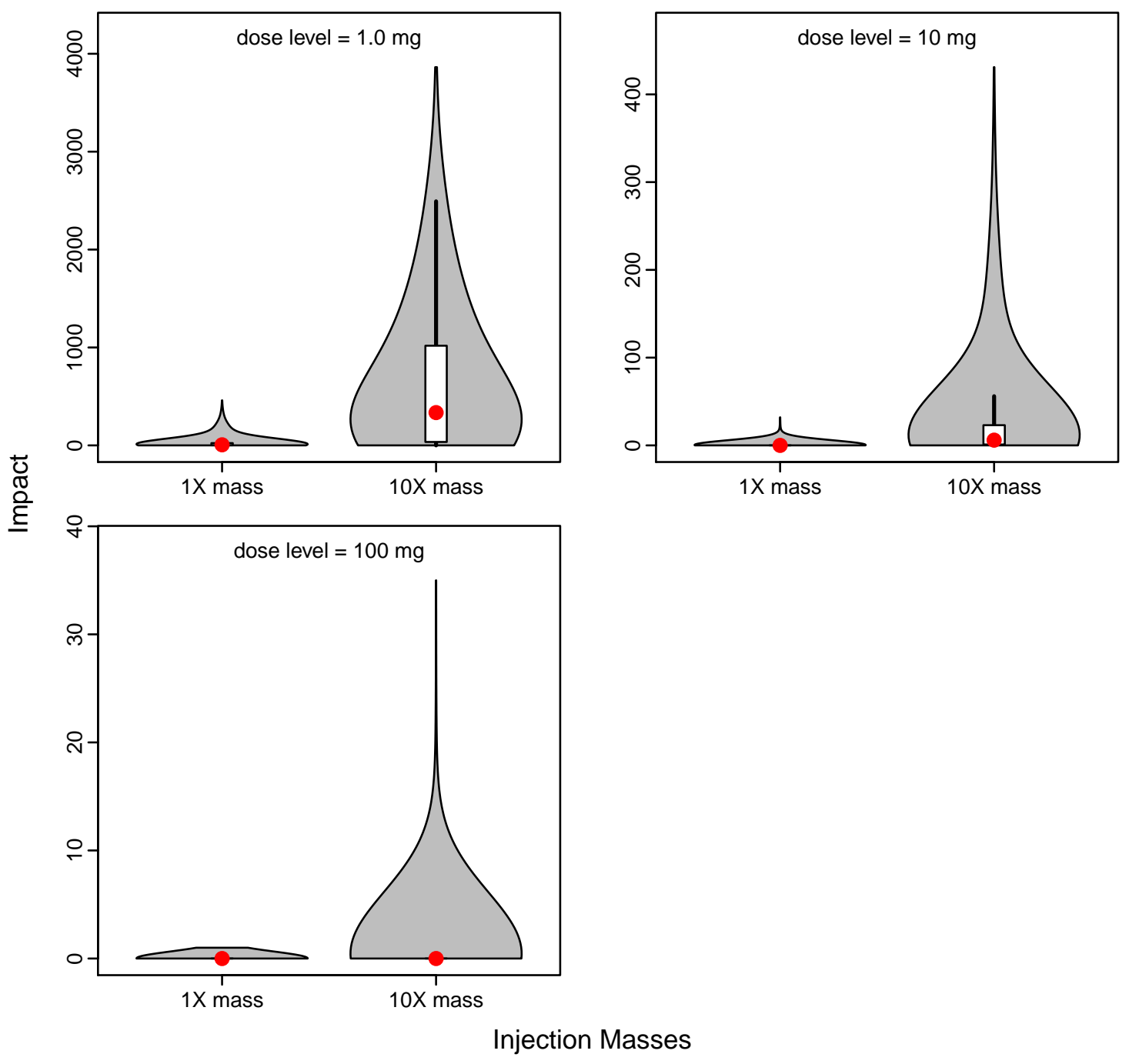

Figure 6.11. Distributions of impacts for Network 12 across all injection nodes for injection masses of $1 \mathrm{X}$ and 10X, for dose levels of 1, 10, and $100 \mathrm{mg}$. 


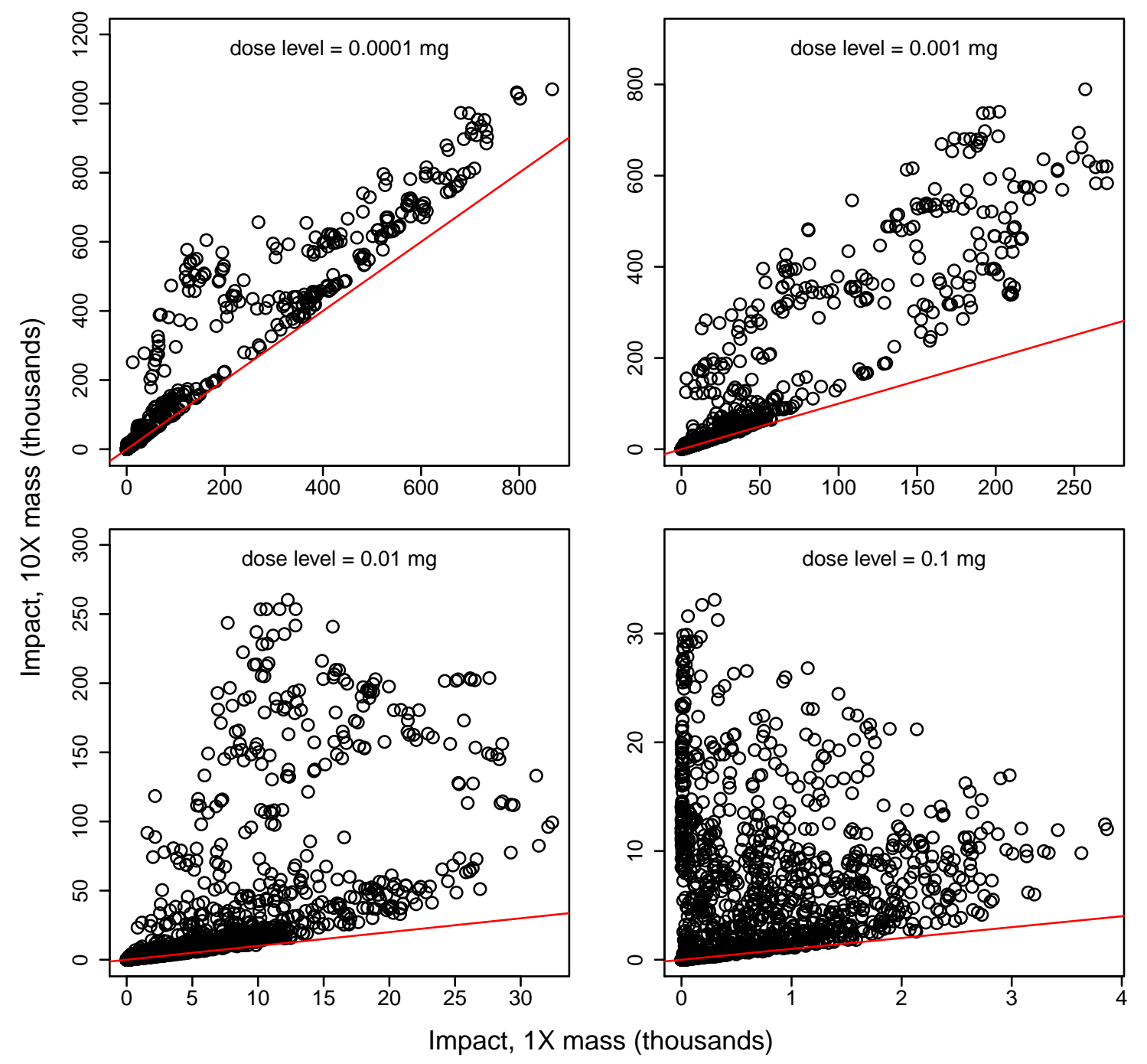

Figure 6.12. Impacts for Network 12 for injection masses of $1 \mathrm{X}$ and $10 \mathrm{X}$, for dose levels of $0.0001,0.001,0.01$, and $0.1 \mathrm{mg}$. The red lines have a slope of 1 . Each point corresponds to one injection node. 


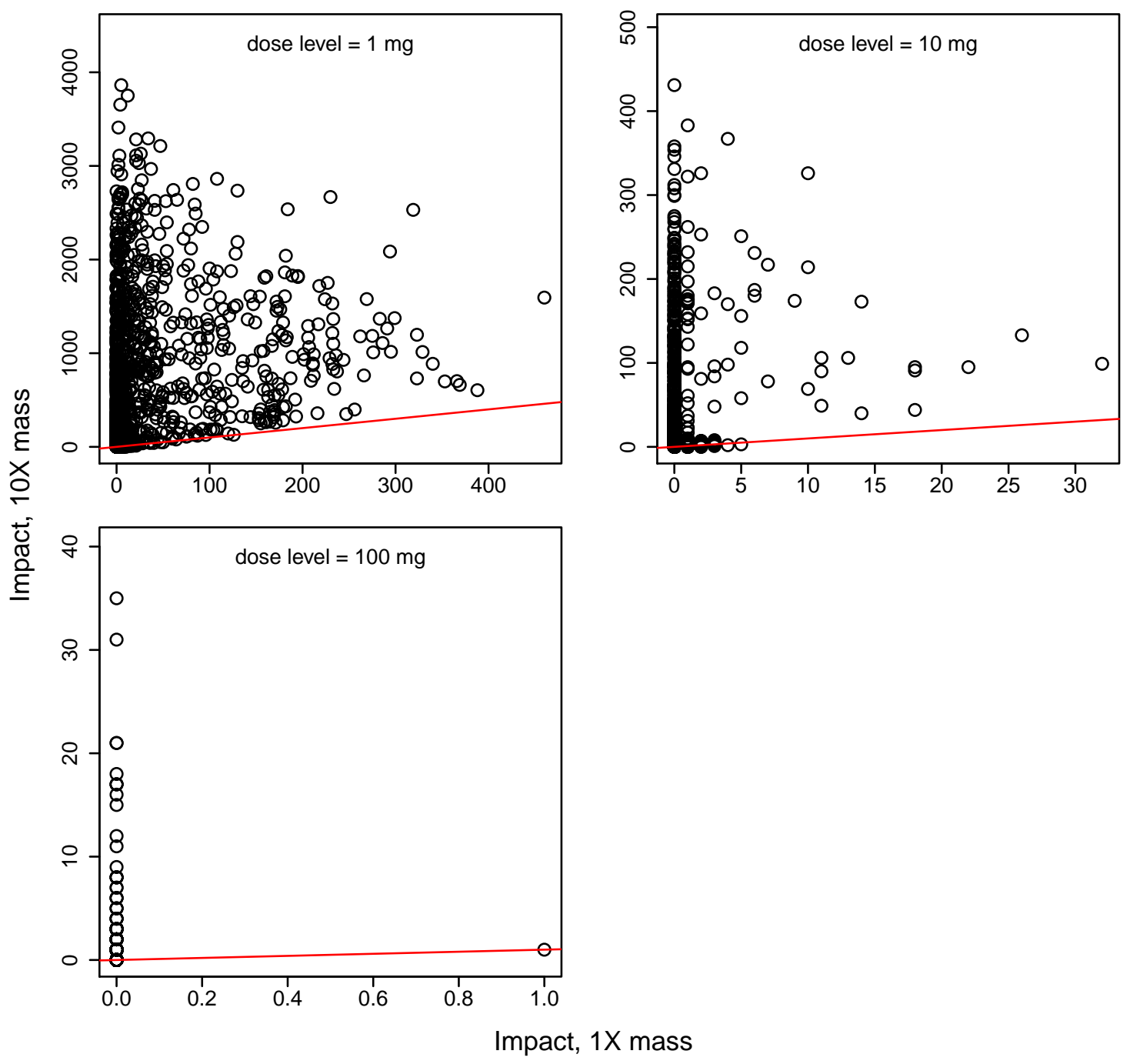

Figure 6.13. Impacts for Network 12 for injection masses of $1 \mathrm{X}$ and $10 \mathrm{X}$, for dose levels of 1,10 , and $100 \mathrm{mg}$. The red lines have a slope of 1 . Each point corresponds to one injection node. 


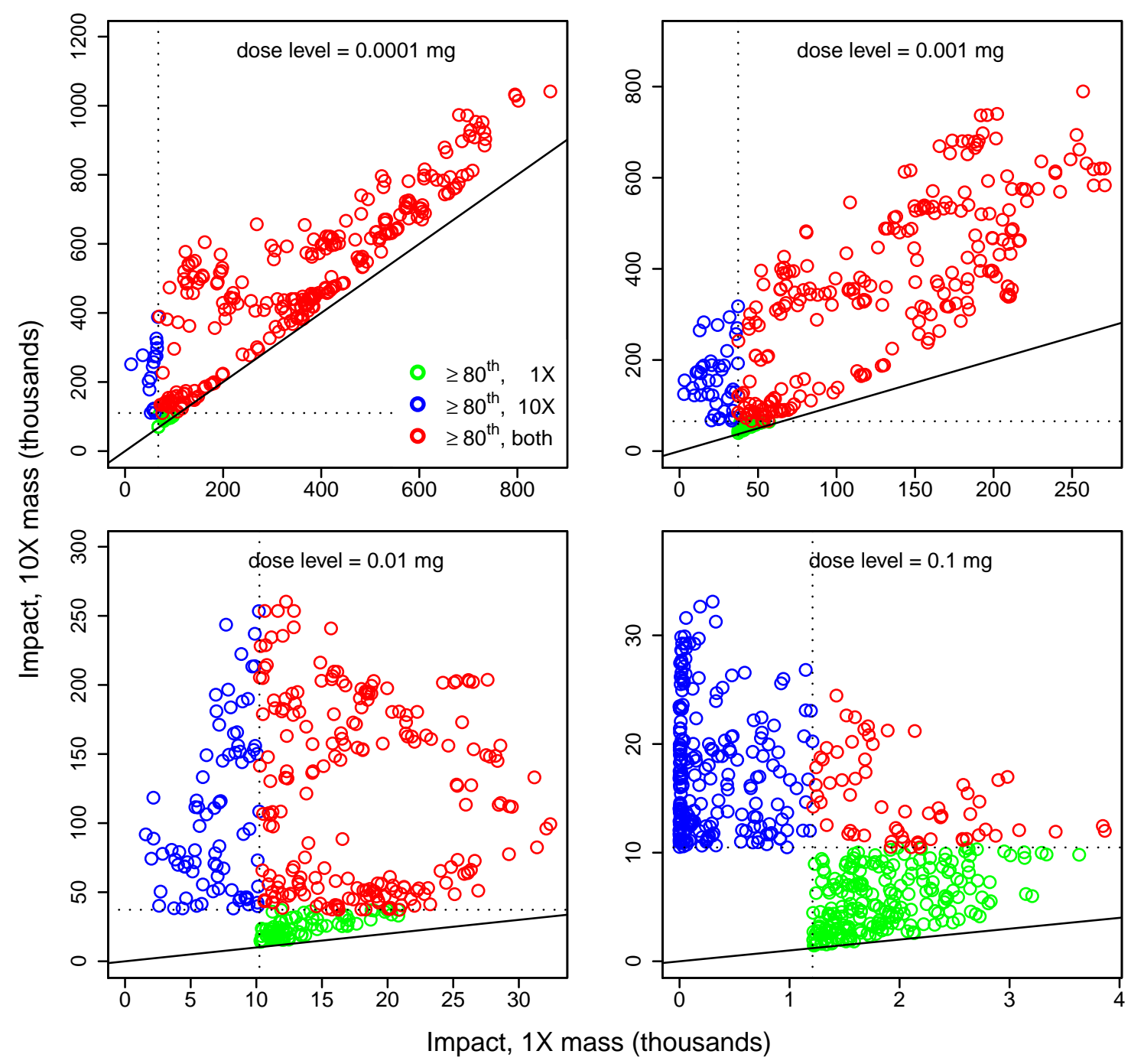

Figure 6.14. Impacts for Network 12 that are at or above the $80^{\text {th }}$ percentile level for $1 \mathrm{X}$ and/or 10X injection masses, for dose levels of $0.0001,0.001,0.01$, and $0.1 \mathrm{mg}$. The dotted lines indicate $80^{\text {th }}$ percentile impacts. The black lines have a slope of 1 . Each point corresponds to one injection node. 


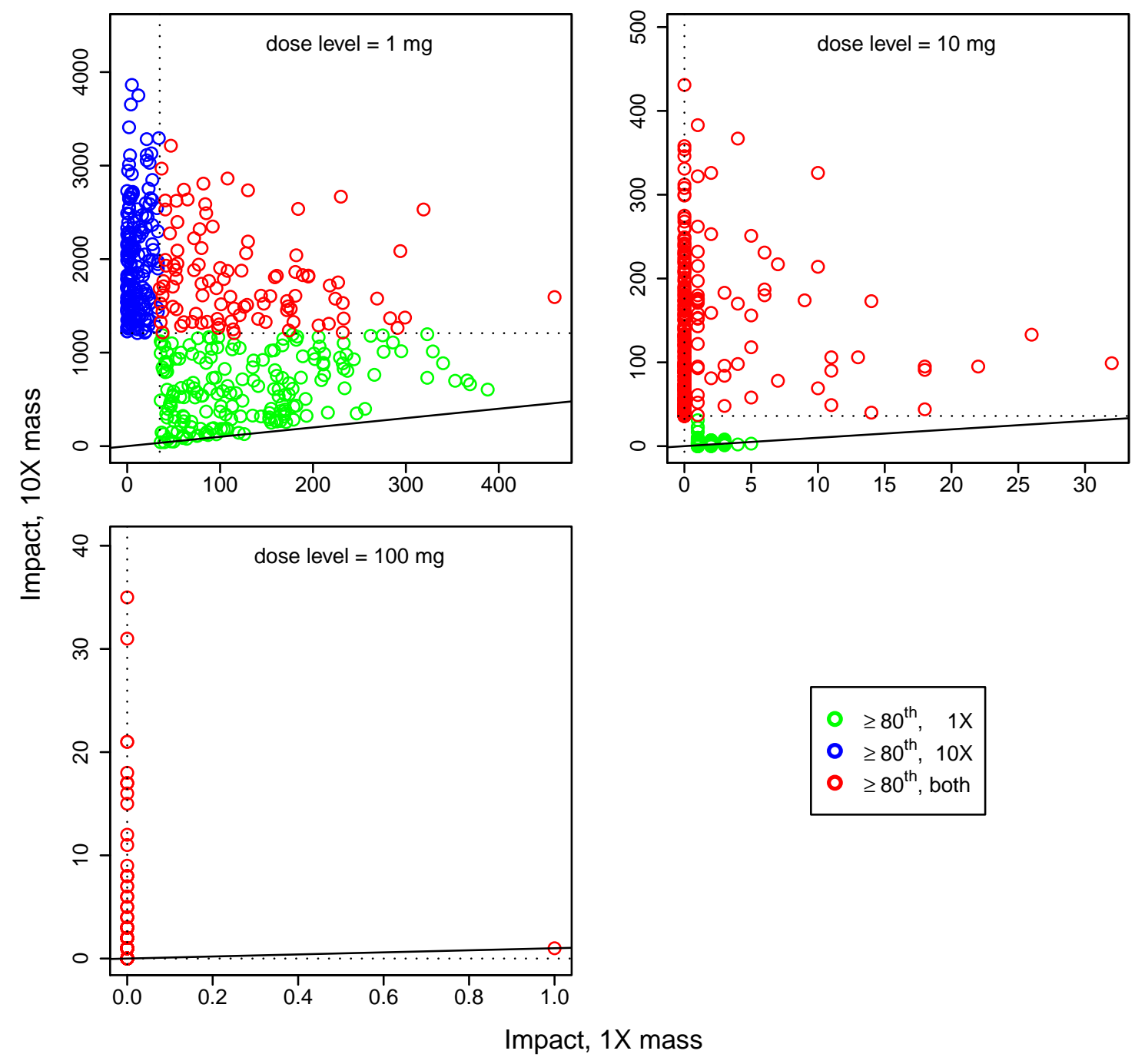

Figure 6.15. Impacts for Network 12 that are at or above the $80^{\text {th }}$ percentile level for $1 \mathrm{X}$ and/or 10X injection masses, for dose levels of 1,10 , and $100 \mathrm{mg}$. The dotted lines indicate $8^{\text {th }}$ percentile impacts. The black lines have a slope of 1 . Each point corresponds to one injection node. 


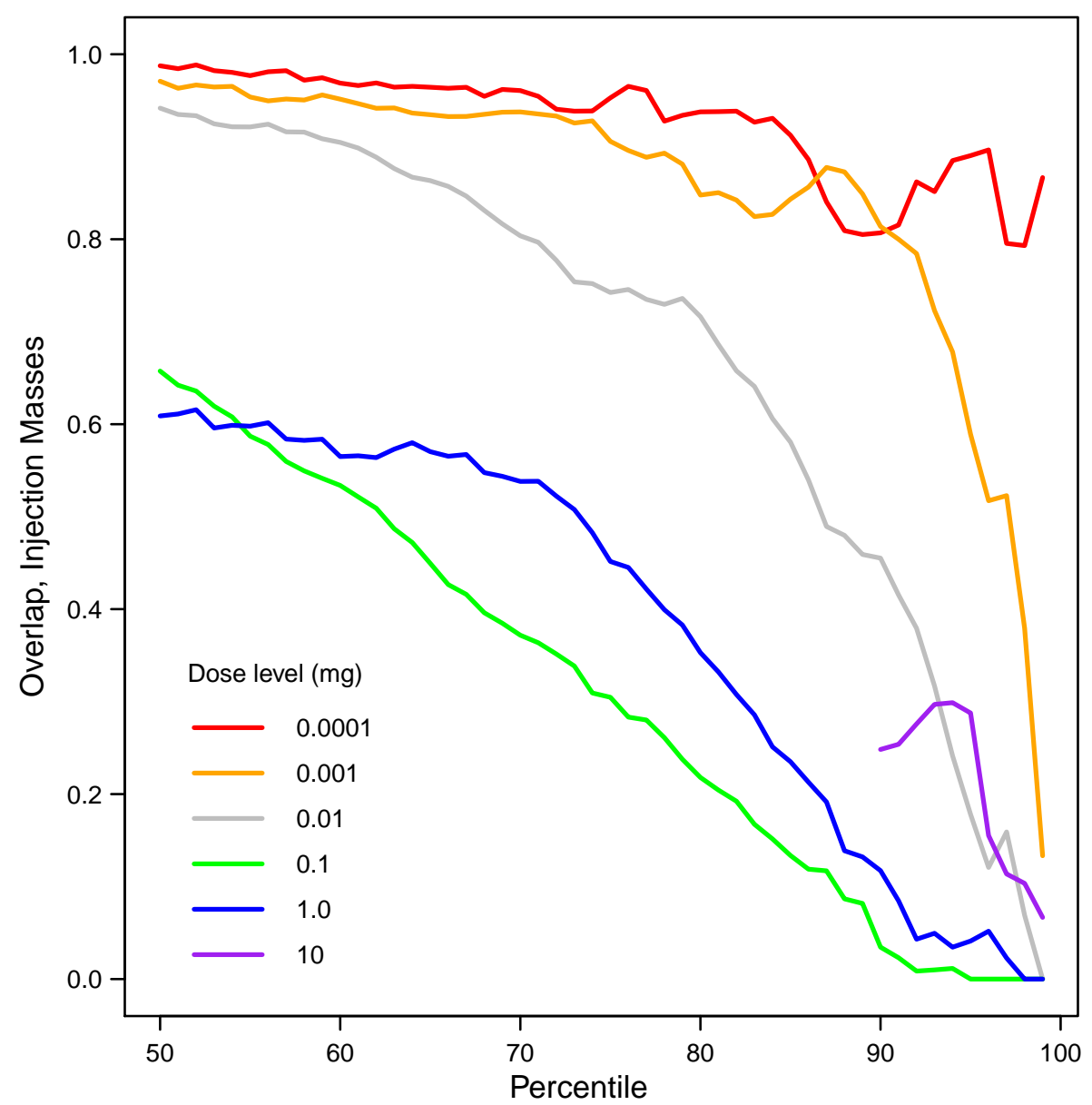

Figure 6.16. Overlap of high percentile injection nodes for Network 12 for injection masses of $1 \mathrm{X}$ and 10X for different dose levels. 

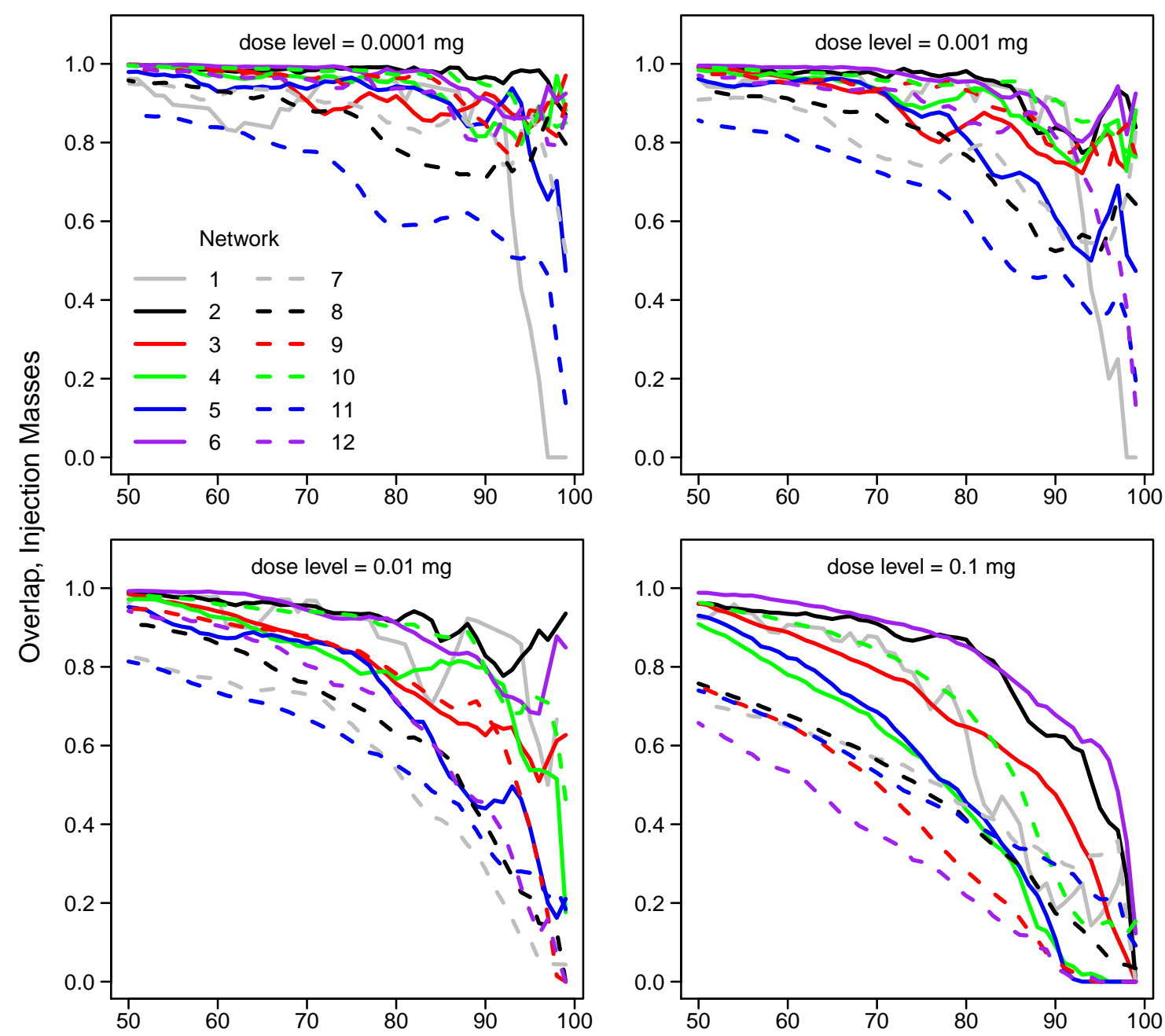

Percentile

Figure 6.17. Overlap of high percentile injection nodes for the networks for injection masses of $1 X$ and 10X, for dose levels of $0.0001,0.001,0.01$, and $0.1 \mathrm{mg}$. 


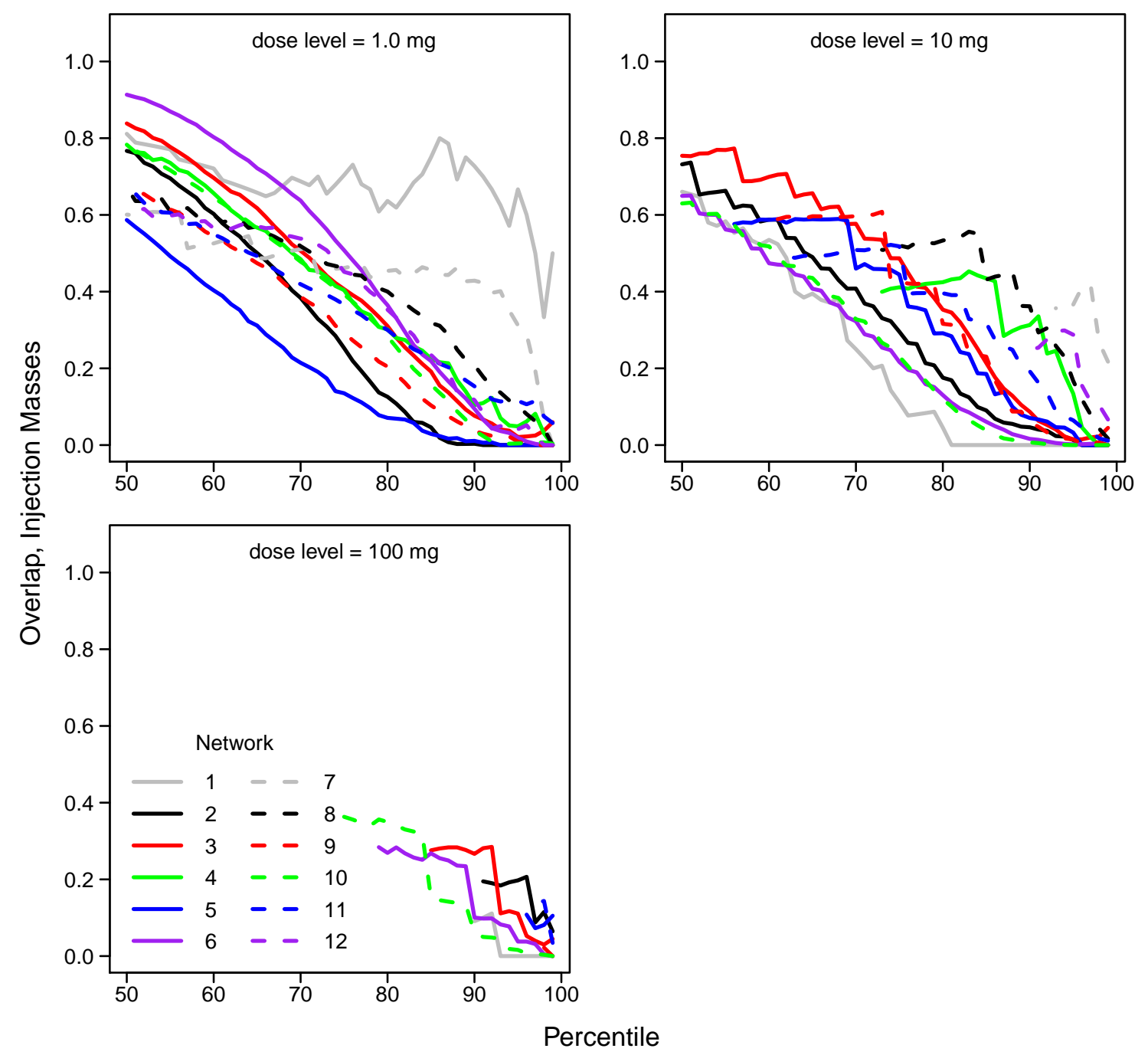

Figure 6.18. Overlap of high percentile injection nodes for the networks for injection masses of $1 \mathrm{X}$ and $10 \mathrm{X}$, for dose levels of 1,10 , and $100 \mathrm{mg}$. 


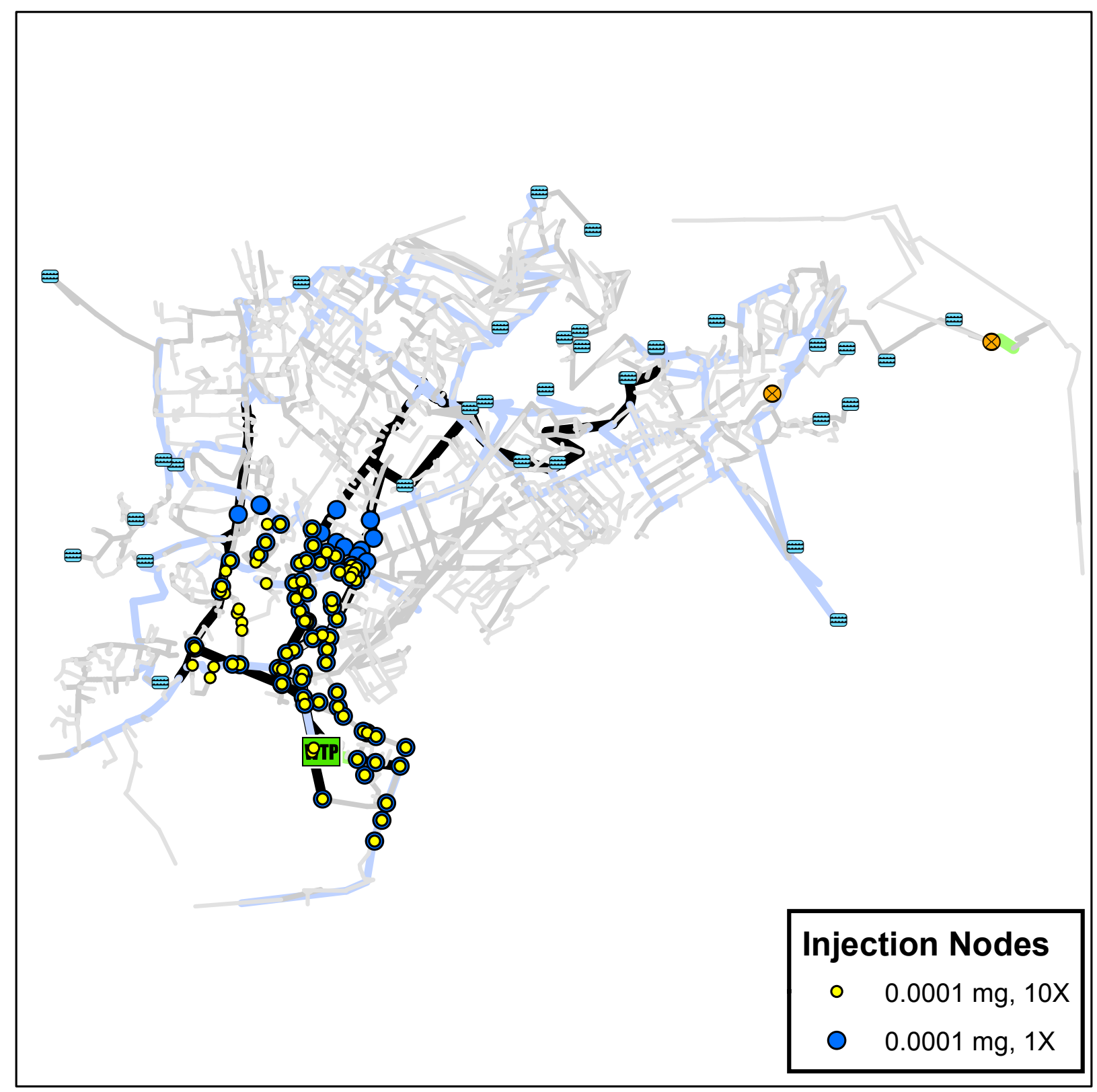

Figure 6.19. Network 4 showing locations of injection nodes associated with $95^{\text {th }}$ percentile or higher impacts at a dose level of $0.0001 \mathrm{mg}$ for injection masses of $1 \mathrm{X}$ and $10 \mathrm{X}$. 


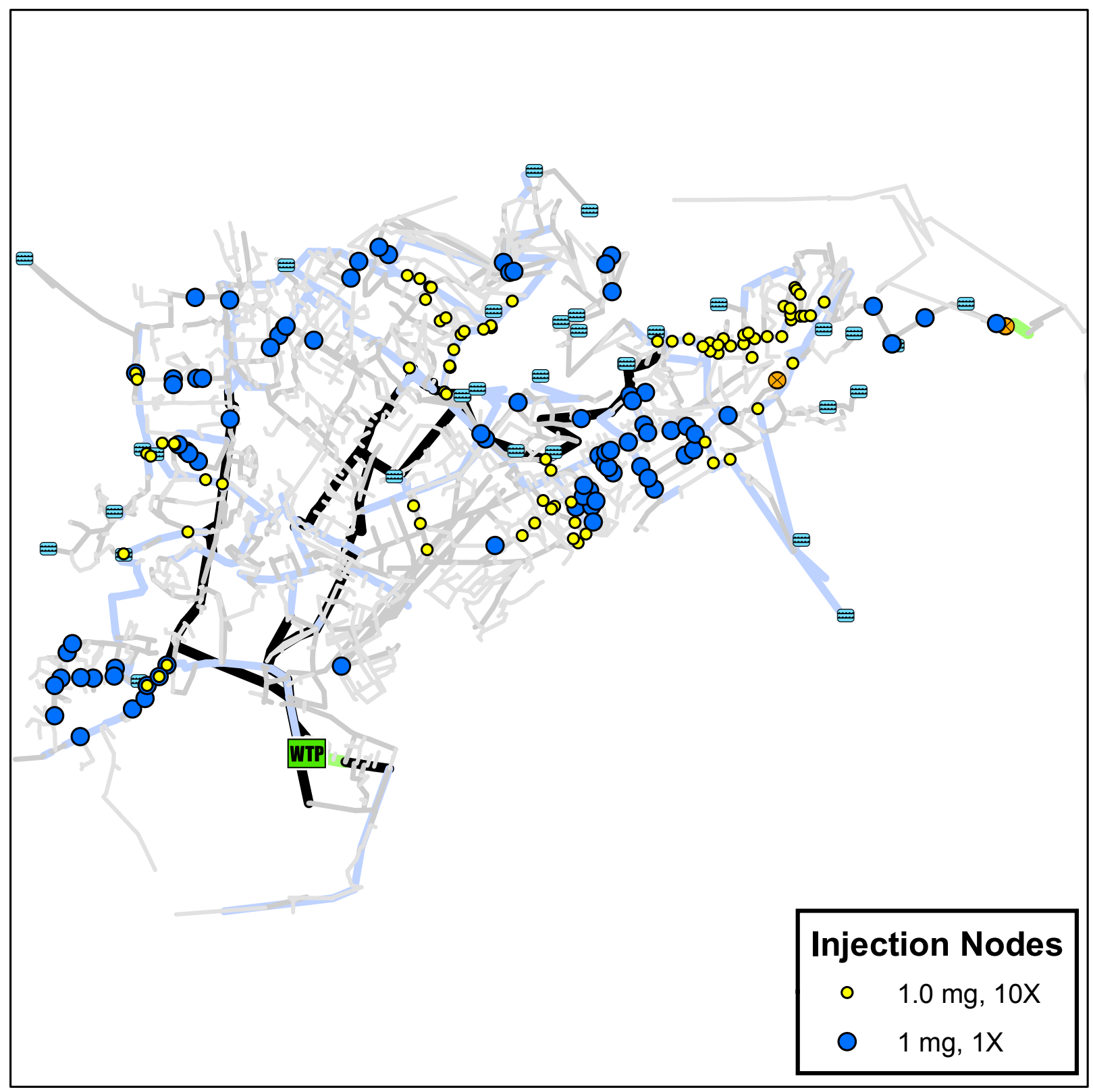

Figure 6.20. Network 4 showing locations of injection nodes associated with $95^{\text {th }}$ percentile or higher impacts at a dose level of $1.0 \mathrm{mg}$ for injection masses of $1 \mathrm{X}$ and $10 \mathrm{X}$. 


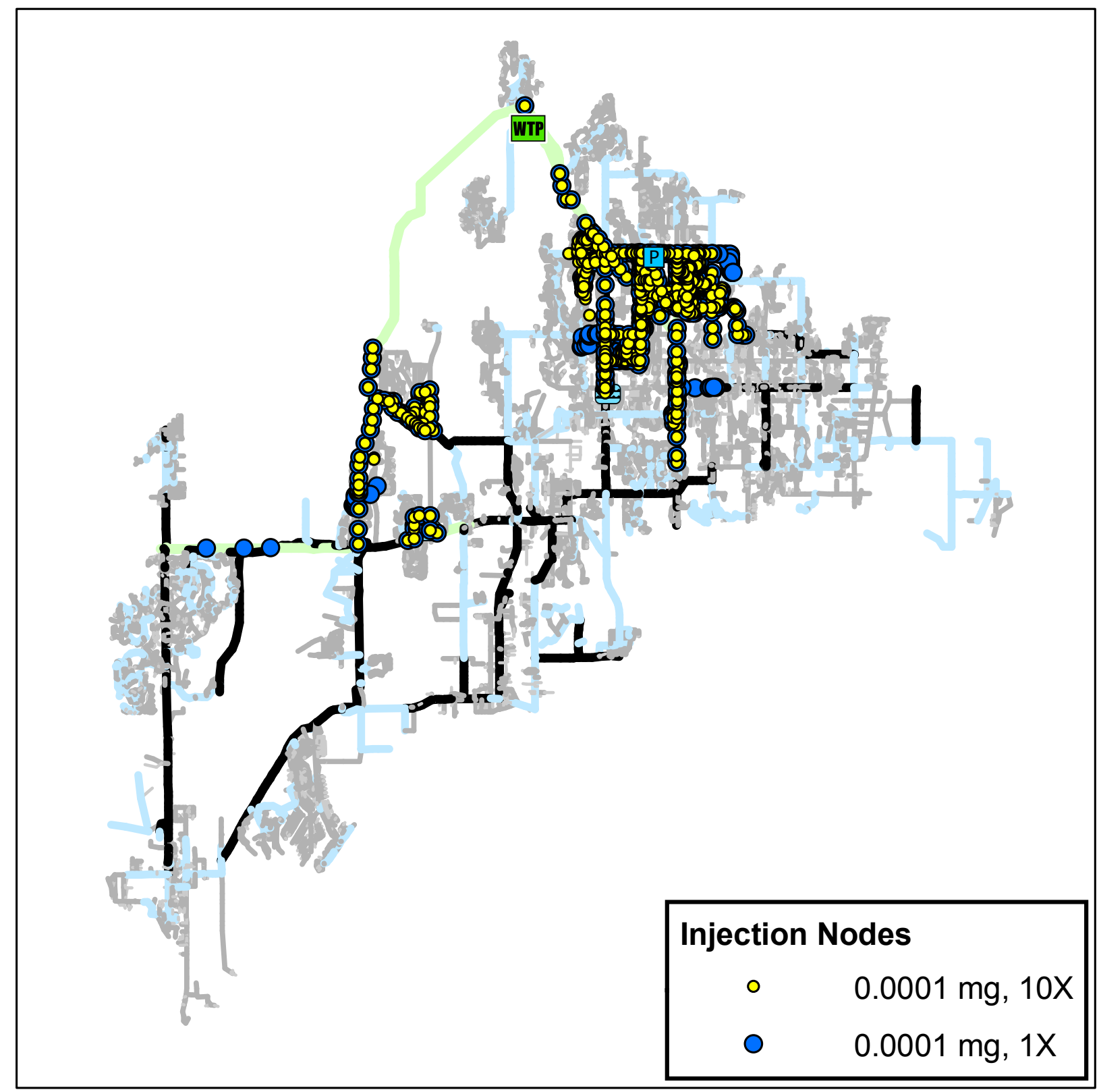

Figure 6.21. Network 6 showing locations of injection nodes associated with $95^{\text {th }}$ percentile or higher impacts at a dose level of $0.0001 \mathrm{mg}$ for injection masses of $1 \mathrm{X}$ and $10 \mathrm{X}$. 


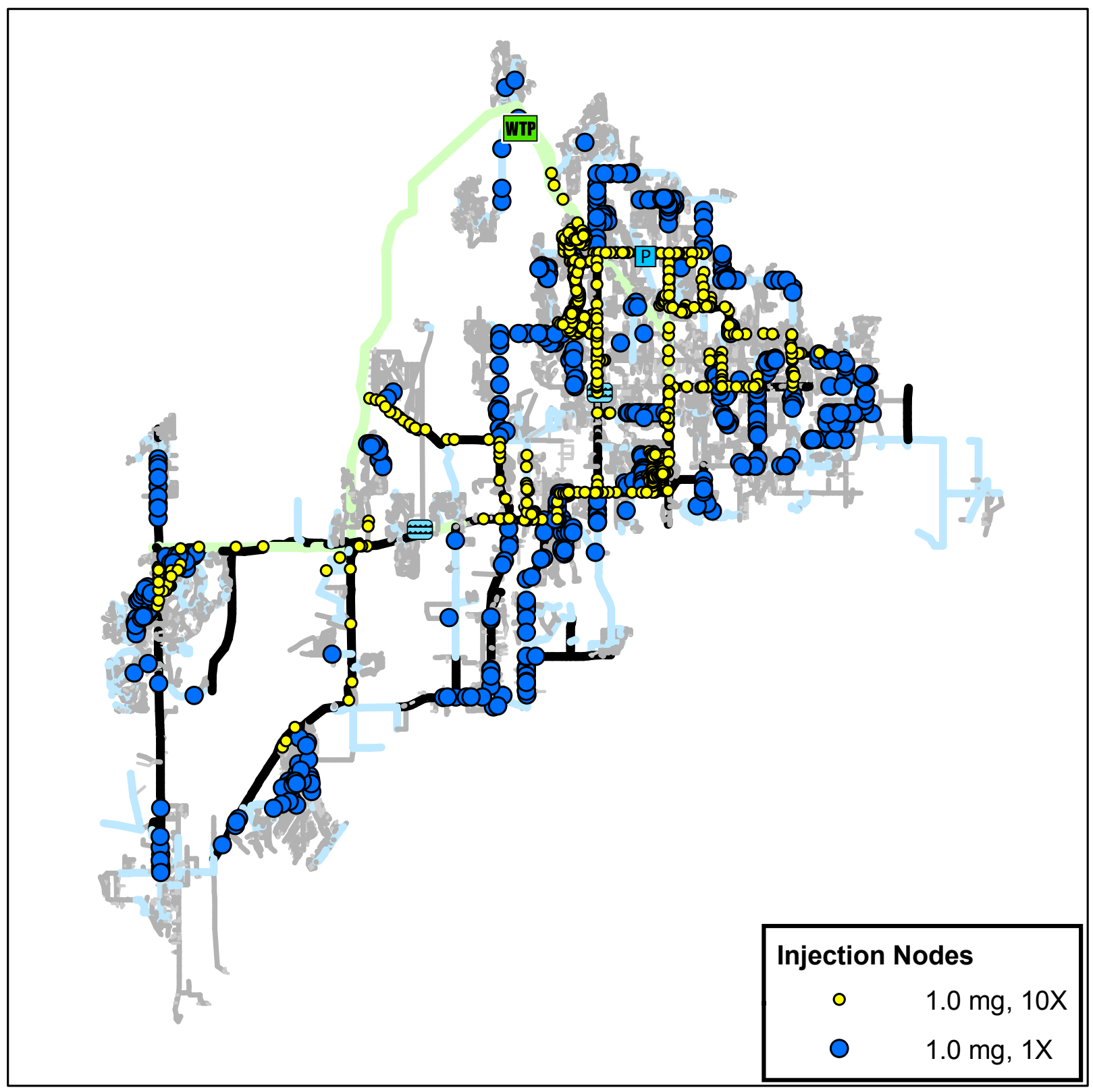

Figure 6.22. Network 6 showing locations of injection nodes associated with $95^{\text {th }}$ percentile or higher impacts at a dose level of $1.0 \mathrm{mg}$ for injection masses of $1 \mathrm{X}$ and $10 \mathrm{X}$. 


\section{Section 7}

\section{Sensitivity of Impacts to Population Distribution}

Two models for population distribution were considered. The first, the base case, uses a demandbased population at each network node. The second uses the same (average) population at each NZD node. The total population for a network is the same for both cases. Figures 7.1 and 7.2 illustrate how the choice of population distribution influences estimated ranked impacts for Network 12. Only for the highest percentile impacts is there any significant difference between impacts for the two distributions and then only for higher dose levels.

The plots in Figures 7.3 and 7.4 provide results for all the networks, many of which show a sensitivity to population distribution similar to that for Network 12. With only a few exceptions, the impacts are not sensitive to the population model for impact percentiles below about $90 \%$. However, for the highest percentiles and higher dose levels, impacts for some of the networks show considerable sensitivity to the population model. This sensitivity occurs because in these cases the injection affects only a small number of nodes and the population assigned to those nodes can have a significant effect on the impact. (For comparison, absolute values for impacts for the networks obtained using the demand-based population model are provided in Figures 3.1 to 3.3.)

The distribution of impacts for Network 12 (Figures 7.5 and 7.6) illustrates that for the lowest dose levels little difference exists between the impacts associated with the two population models. However, as the dose level increases, there is considerable difference between the two cases for the upper tail of the distribution of impacts, consistent with the plots in Figures 7.1 and 7.2.

The scatter plots in Figures 7.7 and 7.8 compare impacts node-by-node for Network 12 for the two different population models. Scatter increases as the dose level increases. The overlap of high percentile injection nodes generally decreases as dose level increases (Figures 7.9 and 7.10) and generally is low for all dose levels for the highest percentile impacts (Figure 7.11).

Overlap of high percentile nodes for the two population models is shown in Figures 7.12 and 7.13 as a function of impact level for all the networks for different dose levels. For most networks, the overlap decreases at the highest percentile impacts and can be quite low, especially for higher dose levels.

The influence of the population model on the locations determined to be high percentile injection nodes is shown in Figures 7.14 through 7.17 for Networks 4 and 6 for dose levels of 0.0001 and $1.0 \mathrm{mg}$. For Network 4 (Figures 7.14 and 7.15), the choice of population model has limited influence on the locations of nodes at or above the $95^{\text {th }}$ percentile level for either dose level, con- 
sistent with the overlaps shown in Figures 7.12 and 7.13 for the network (near $100 \%$ for $0.0001 \mathrm{mg}$ and about $65 \%$ for $1.0 \mathrm{mg}$ ). For Network 6 (Figures 7.16 and 7.17), the high percentile injection nodes determined using the two population models are much the same for the $0.0001 \mathrm{mg}$ dose level, but differ considerably for a dose level of $1.0 \mathrm{mg}$. From Figures 7.12 and 7.13, the overlaps for the two population models for the network are about $80 \%$ and $30 \%$ at $0.0001 \mathrm{mg}$ and $1.0 \mathrm{mg}$, respectively, which is consistent with the maps.

Impacts can have considerable sensitivity to population distribution at high dose levels, if the impacts have very high rankings (greater than about the $95^{\text {th }}$ percentile), with considerable variability among the networks. Overlap of high percentile injection nodes can be low for the two different population models, especially at high dose levels. 


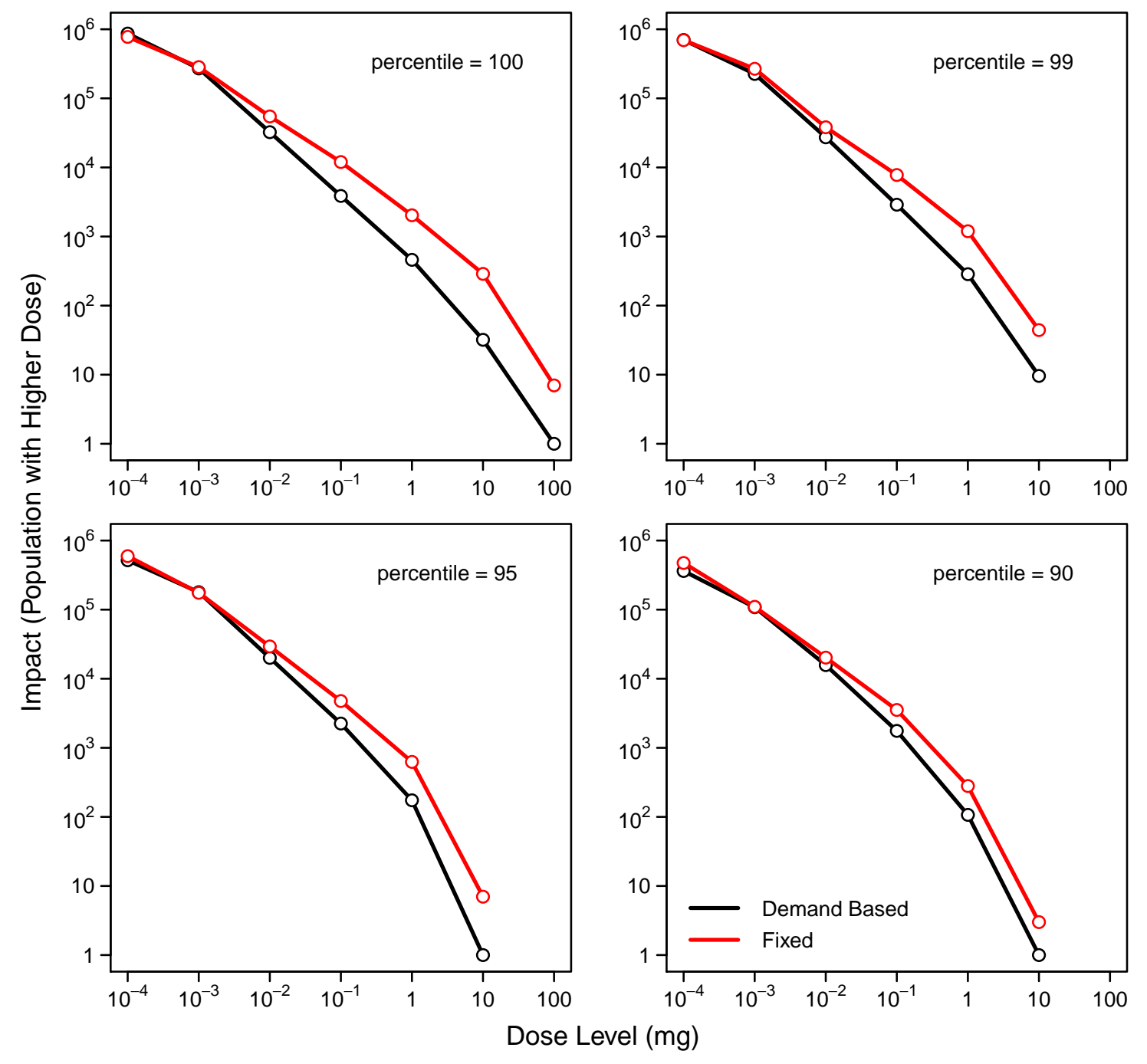

Figure 7.1. Impacts associated with different population models for Network 12 as a function of dose level, for the $90^{\text {th }}, 95^{\text {th }}, 99^{\text {th }}$, and $100^{\text {th }}$ percentile injection nodes. 


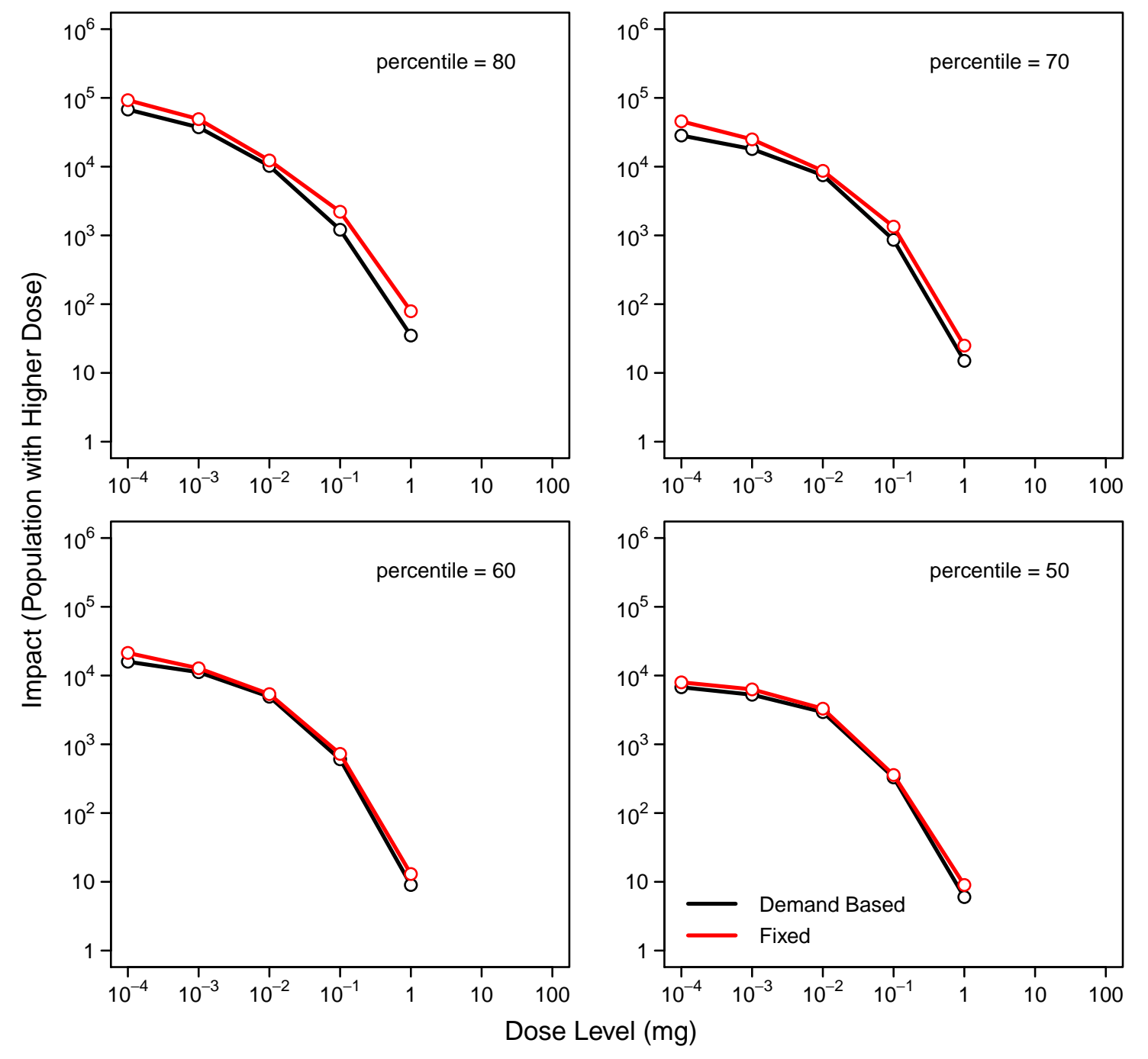

Figure 7.2. Impacts associated with different population models for Network 12 as a function of dose level, for the $50^{\text {th }}, 60^{\text {th }}, 70^{\text {th }}$, and $80^{\text {th }}$ percentile injection nodes. 


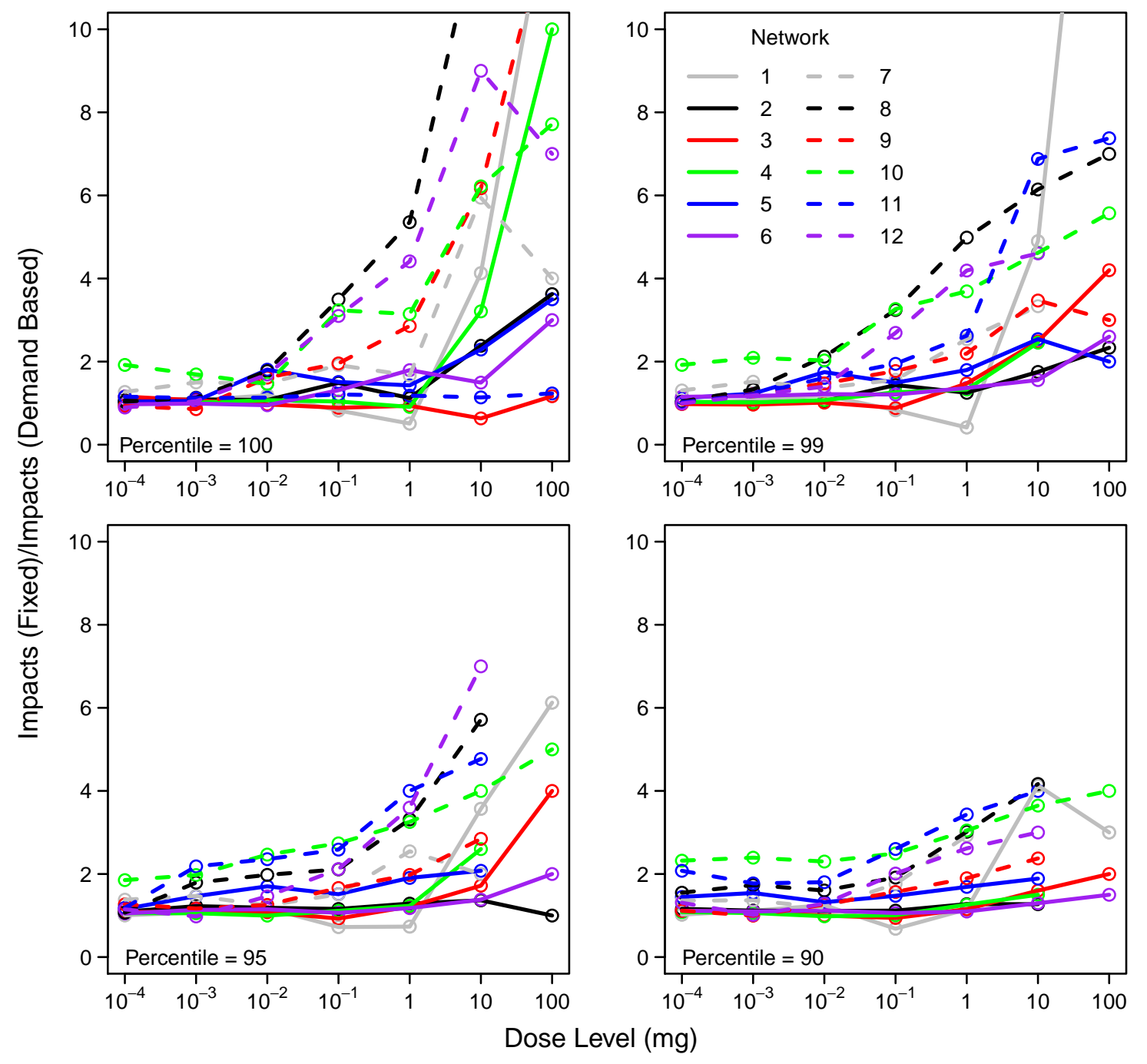

Figure 7.3. Relative magnitudes of the impacts for the networks obtained with different population models as a function of dose level, for the $90^{\text {th }}, 95^{\text {th }}, 99^{\text {th }}$, and $100^{\text {th }}$ percentile injection nodes. 


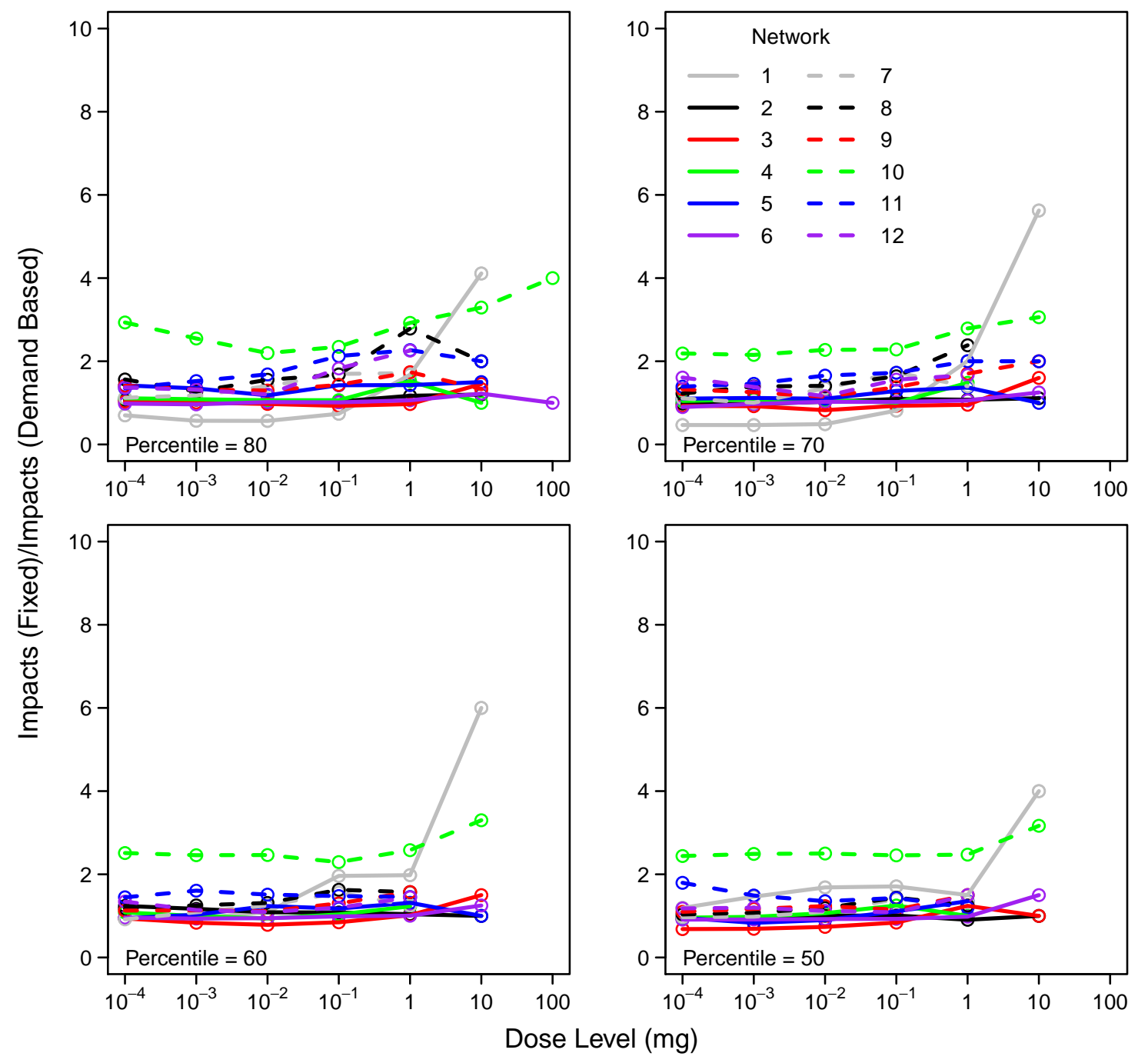

Figure 7.4. Relative magnitudes of the impacts for the networks obtained with different population models as a function of dose level, for the $50^{\text {th }}, 60^{\text {th }}, 70^{\text {th }}$, and $80^{\text {th }}$ percentile injection nodes. 


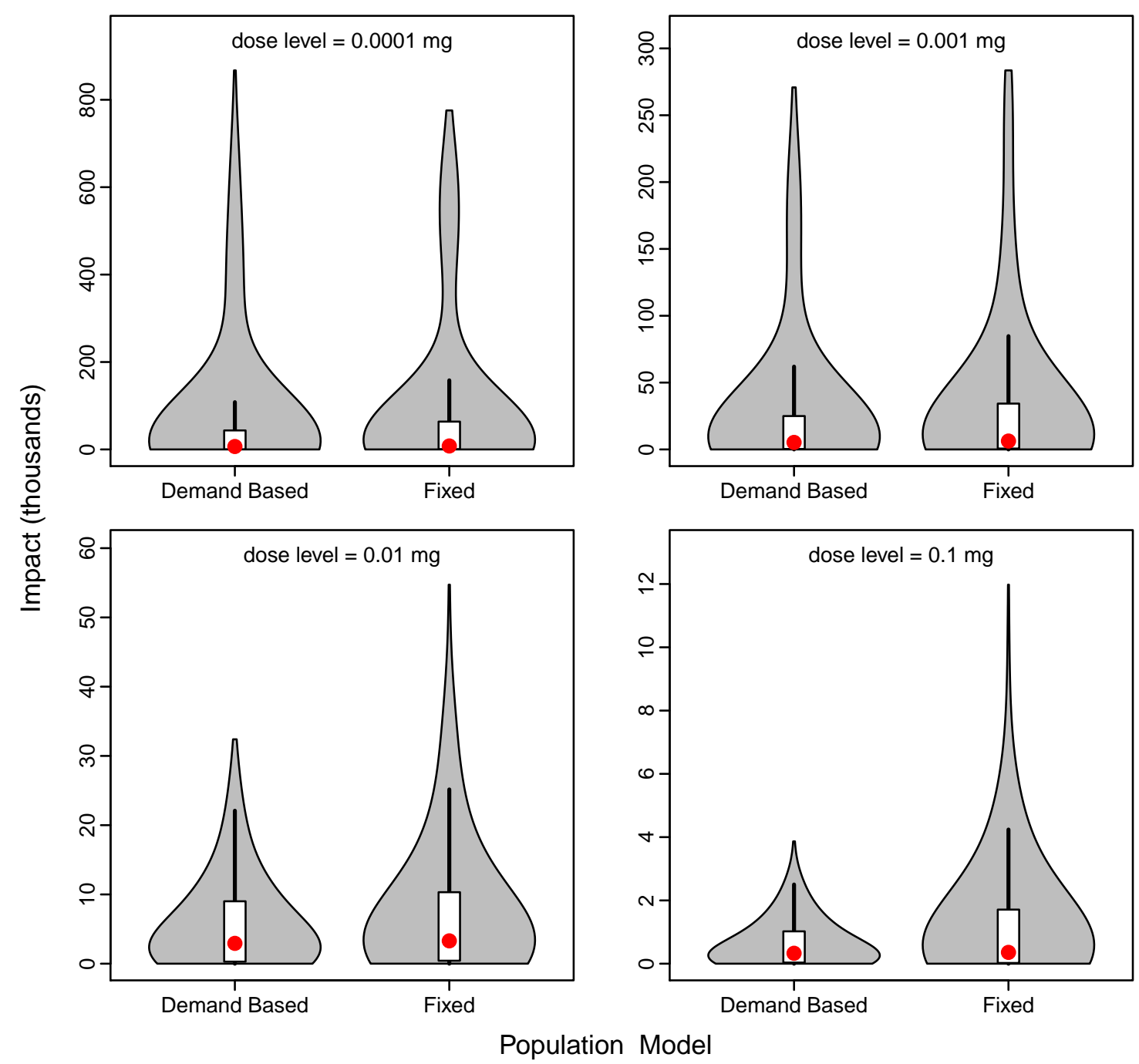

Figure 7.5. Distributions of impacts for Network 12 across all injection nodes for different population models, for dose levels of $0.0001,0.001,0.01$, and $0.1 \mathrm{mg}$. 


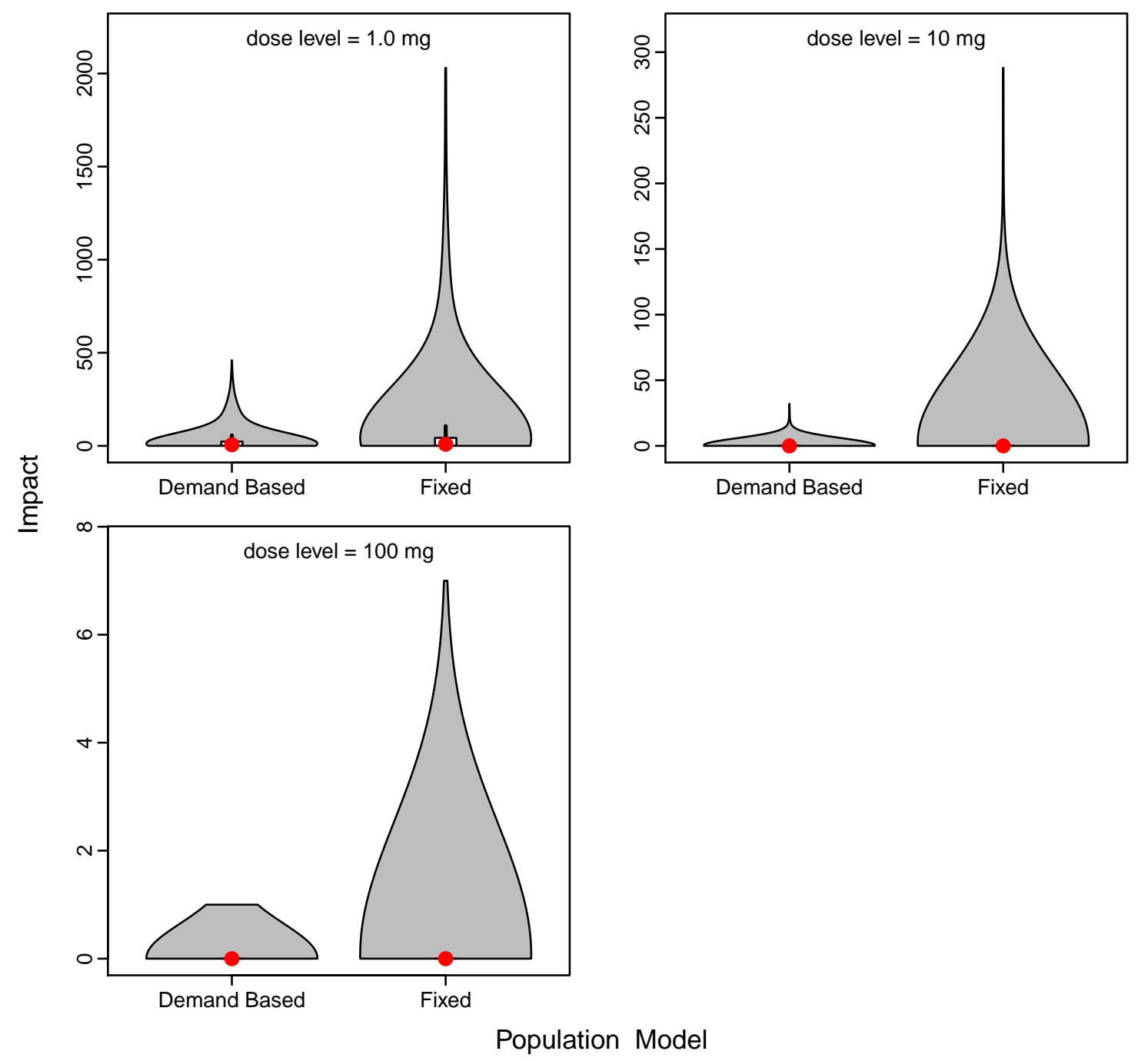

Figure 7.6. Distributions of impacts for Network 12 across all injection nodes for different population models, for dose levels of 1,10 , and $100 \mathrm{mg}$. 


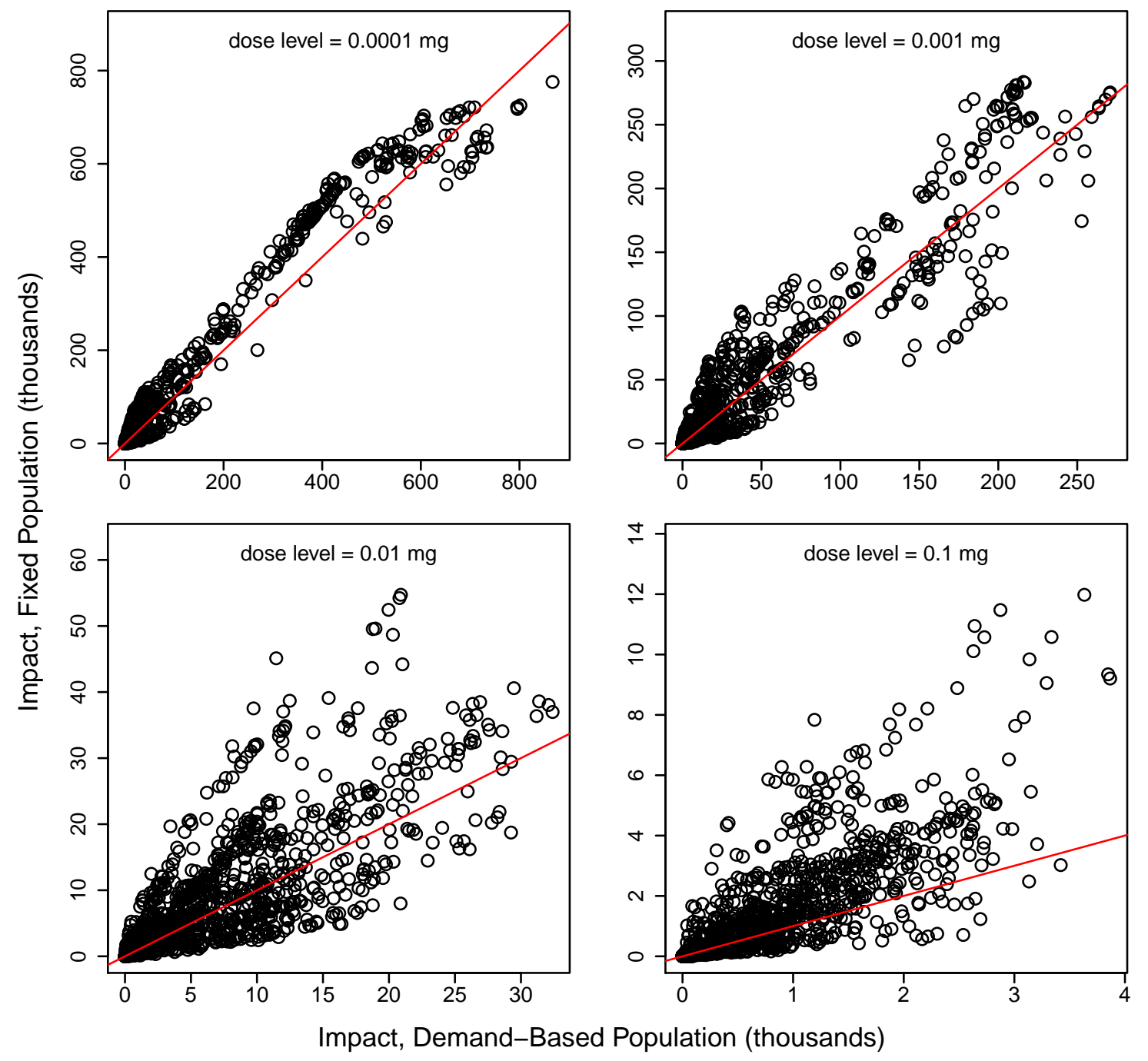

Figure 7.7. Impacts for Network 12 obtained with different population models, for dose levels of $0.0001,0.001,0.01$, and $0.1 \mathrm{mg}$. The red lines have a slope of 1 . Each point corresponds to one injection node. 


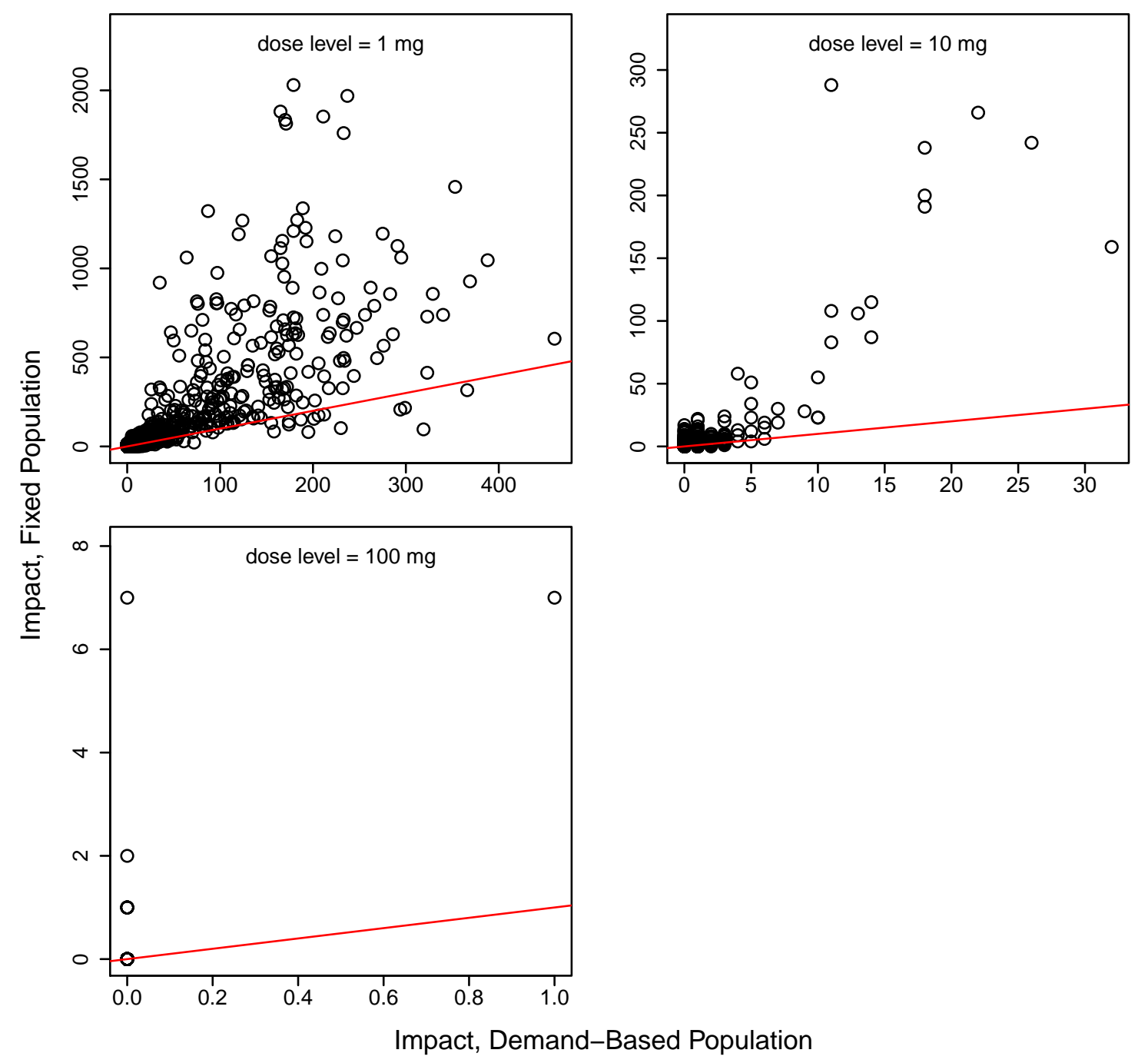

Figure 7.8. Impacts for Network 12 obtained with different population models, for dose levels of 1,10 , and $100 \mathrm{mg}$. The red lines have a slope of 1 . Each point corresponds to one injection node. 


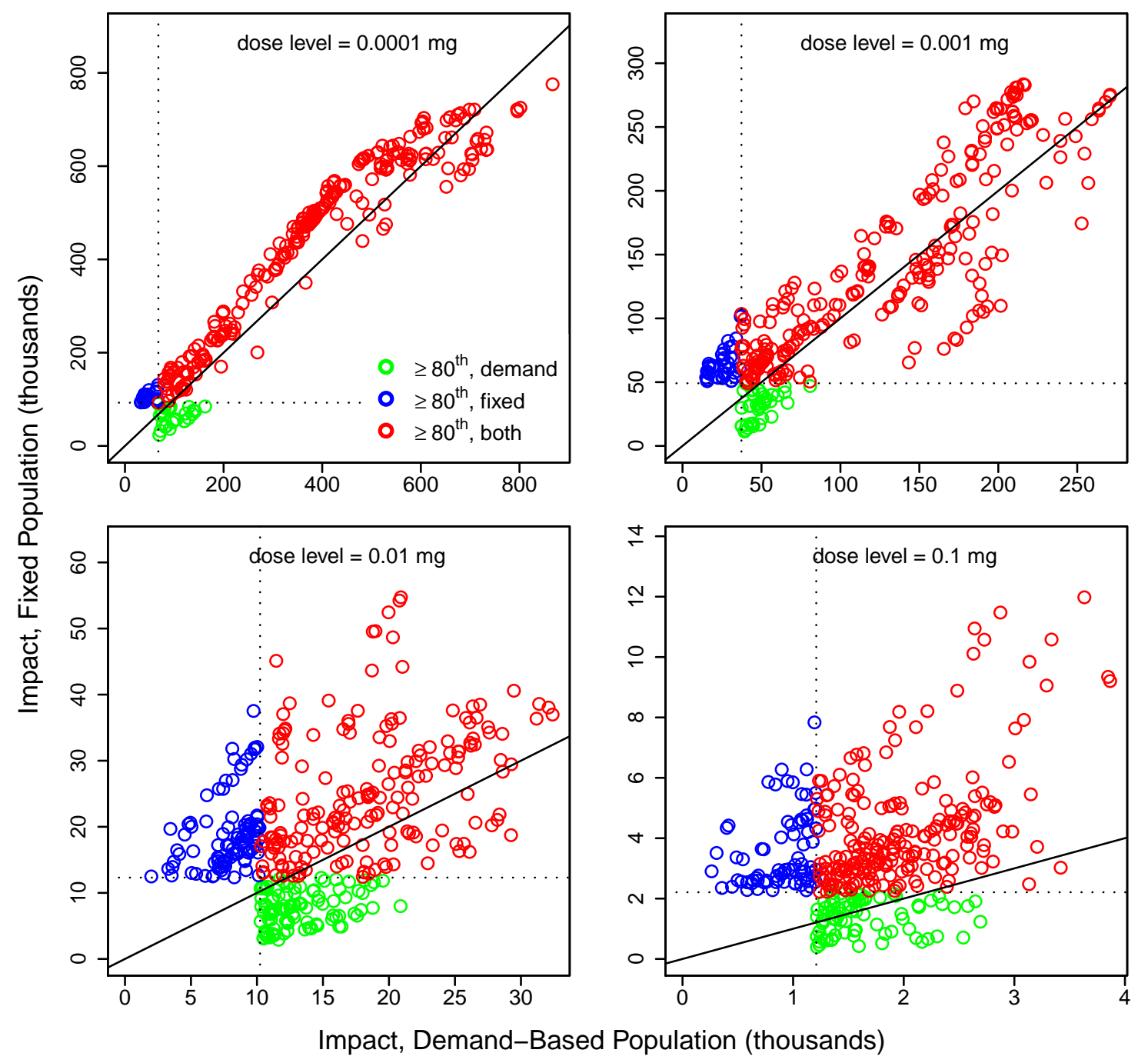

Figure 7.9. Impacts for Network 12 that are at or above the $80^{\text {th }}$ percentile level obtained with different population models, for dose levels of $0.0001,0.001,0.01$, and $0.1 \mathrm{mg}$. The dotted lines indicate $80^{\text {th }}$ percentile impacts. The black lines have a slope of 1 . Each point corresponds to one injection node. 


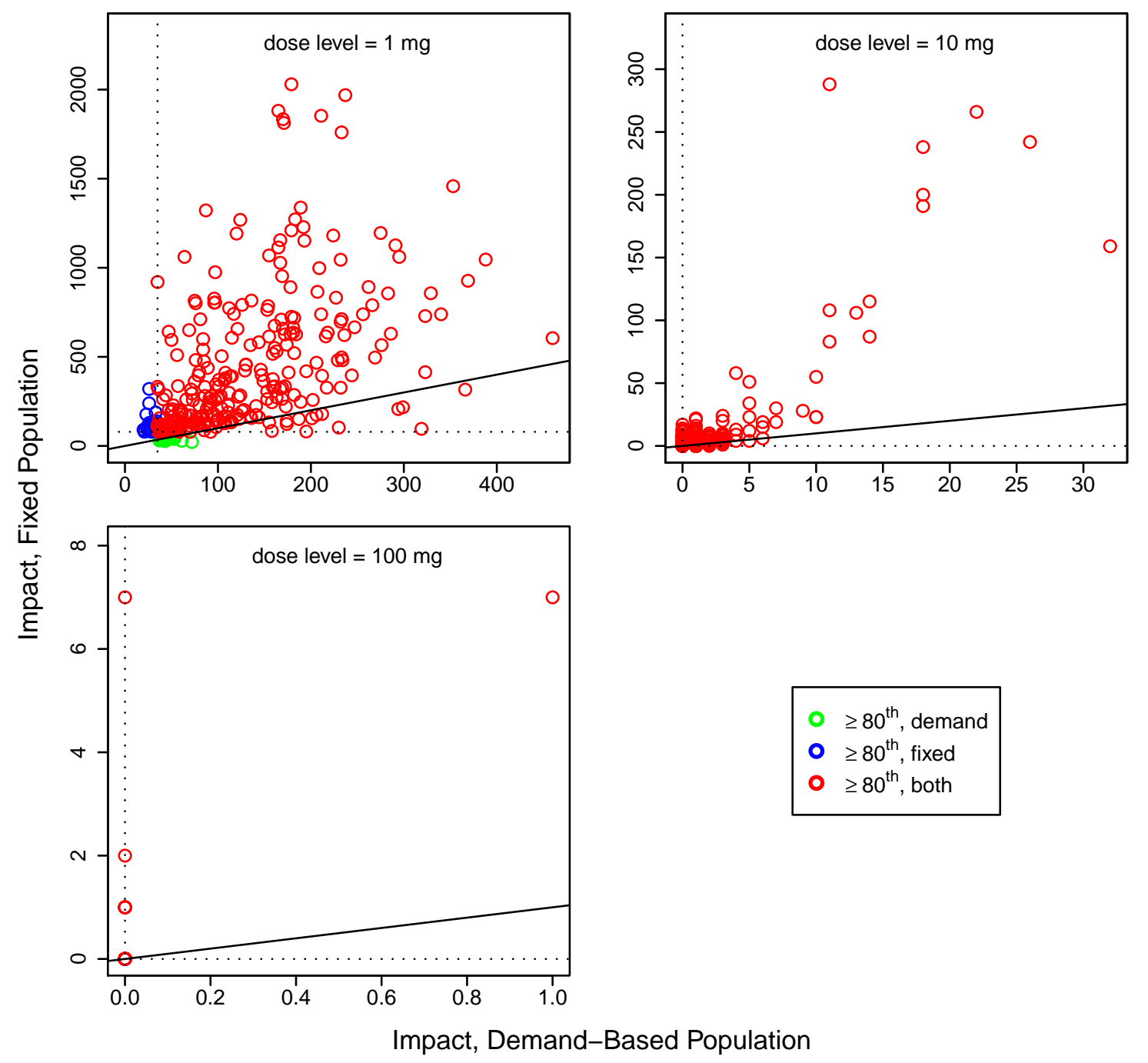

Figure 7.10. Impacts for Network 12 that are at or above the $80^{\text {th }}$ percentile level obtained with different population models, for dose levels of 1,10 , and $100 \mathrm{mg}$. The dotted lines indicate $80^{\text {th }}$ percentile impacts. The black lines have a slope of 1 . Each point corresponds to one injection node. 


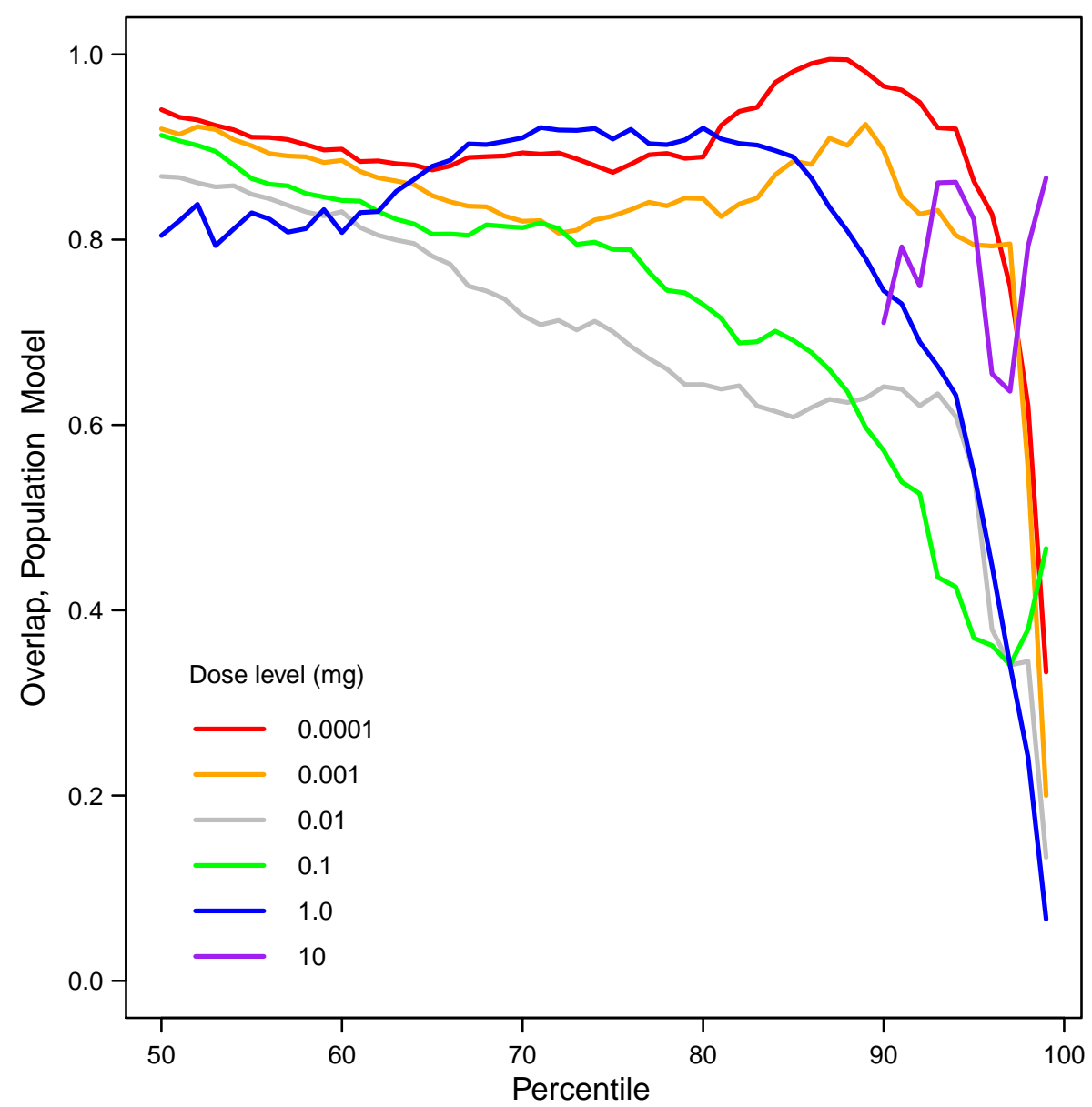

Figure 7.11. Overlap of high percentile injection nodes for Network 12 for the two population models for different dose levels. 

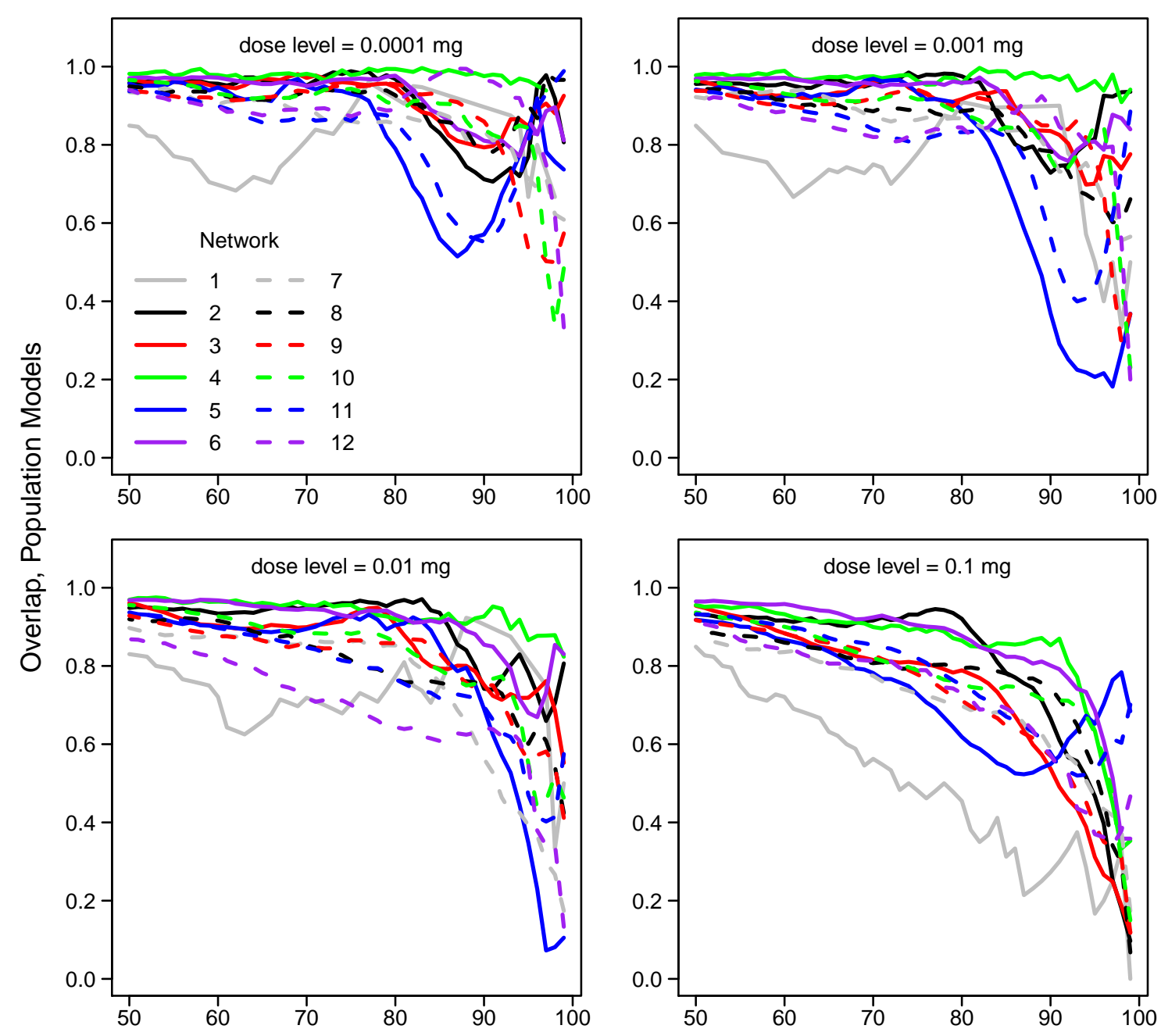

Percentile

Figure 7.12. Overlap of high percentile injection nodes for the networks for the two population models, for dose levels of $0.0001,0.001,0.01$, and $0.1 \mathrm{mg}$. 


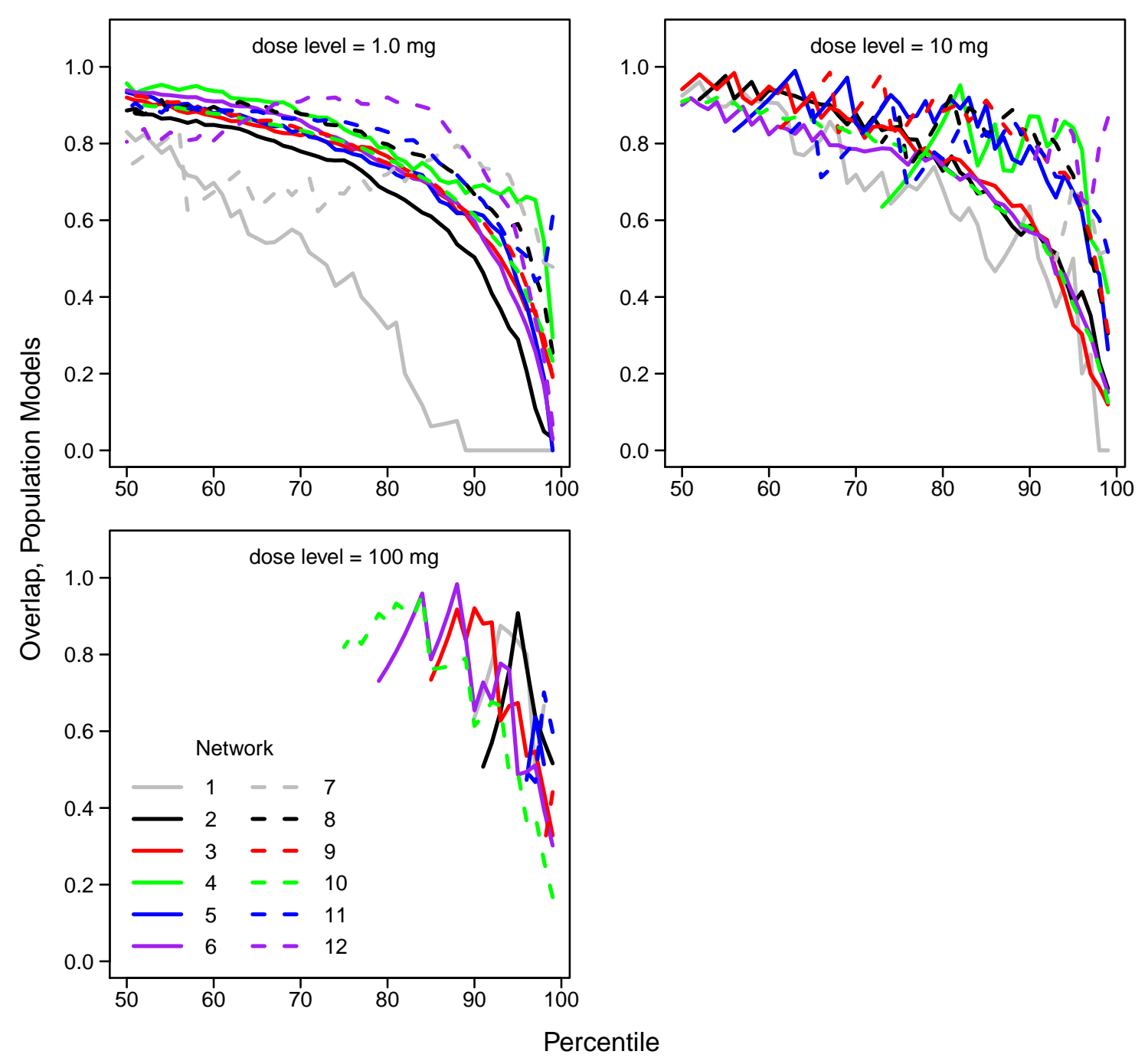

Figure 7.13. Overlap of high percentile injection nodes for the networks for the two population models, for dose levels of 1, 10, and $100 \mathrm{mg}$. 


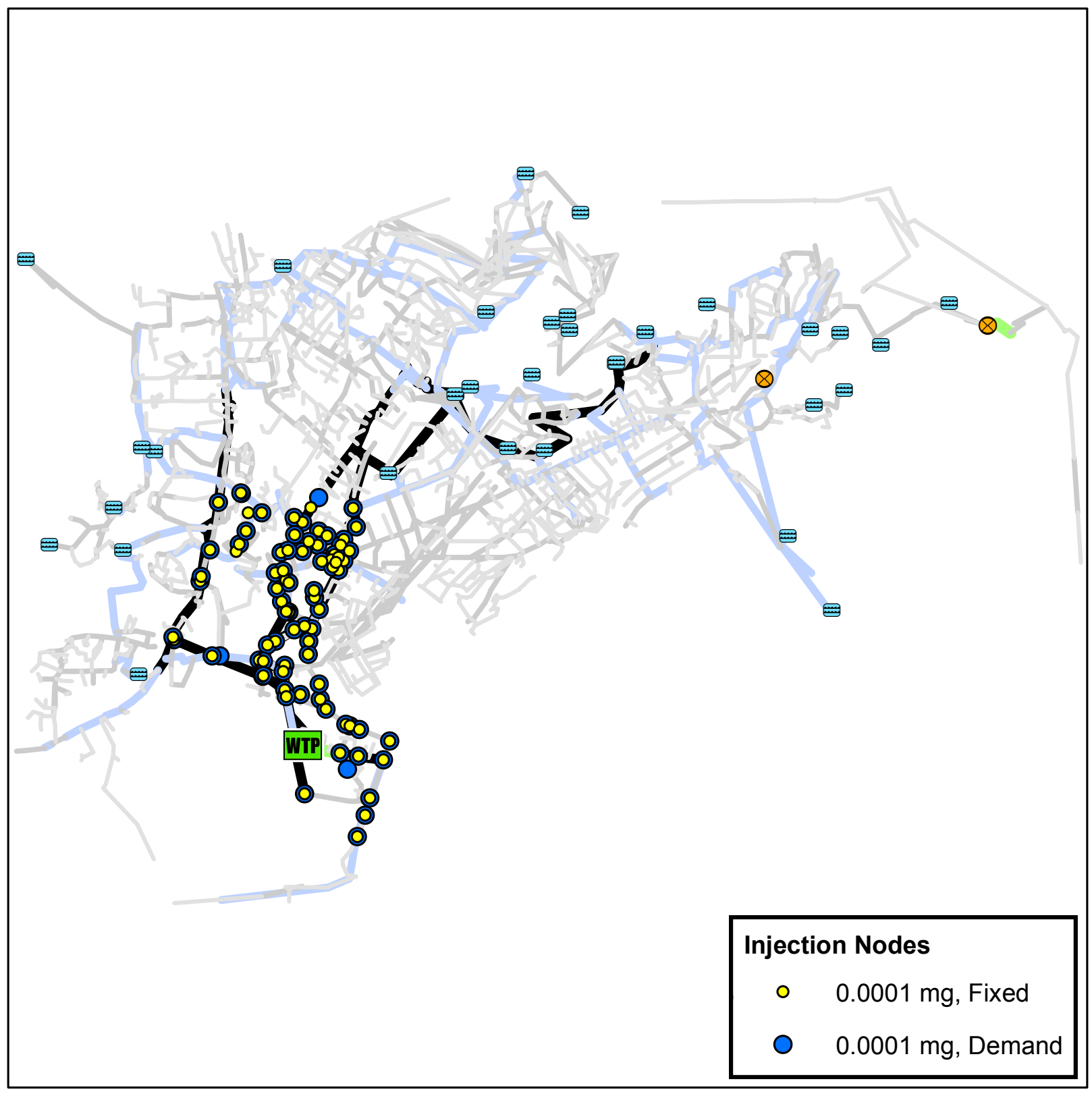

Figure 7.14. Network 4 showing locations of injection nodes associated with $95^{\text {th }}$ percentile or higher impacts at a dose level of $0.0001 \mathrm{mg}$ for the two population models. 


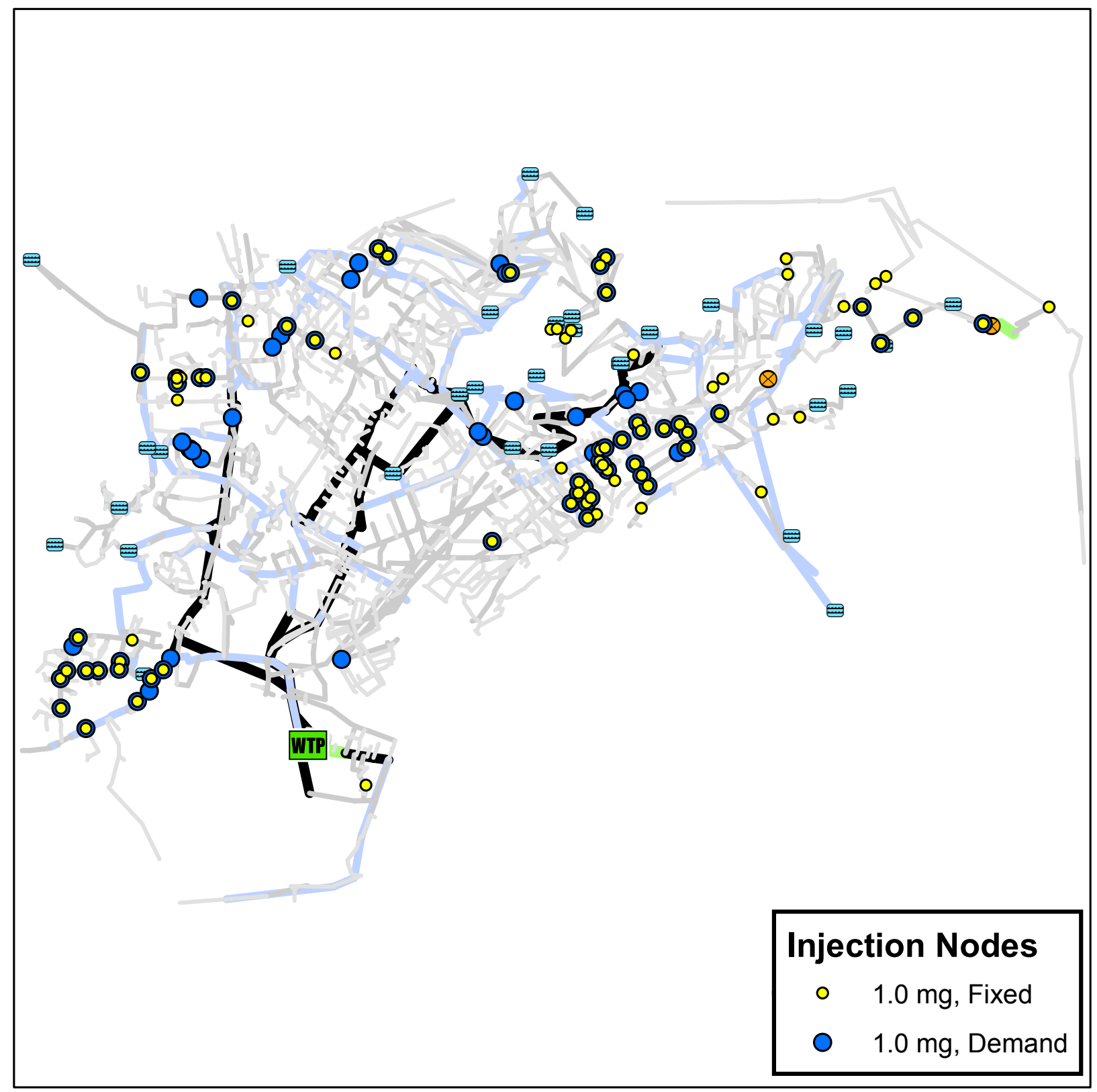

Figure 7.15. Network 4 showing locations of injection nodes associated with $95^{\text {th }}$ percentile or higher impacts at a dose level of $1.0 \mathrm{mg}$ for the two population models. 


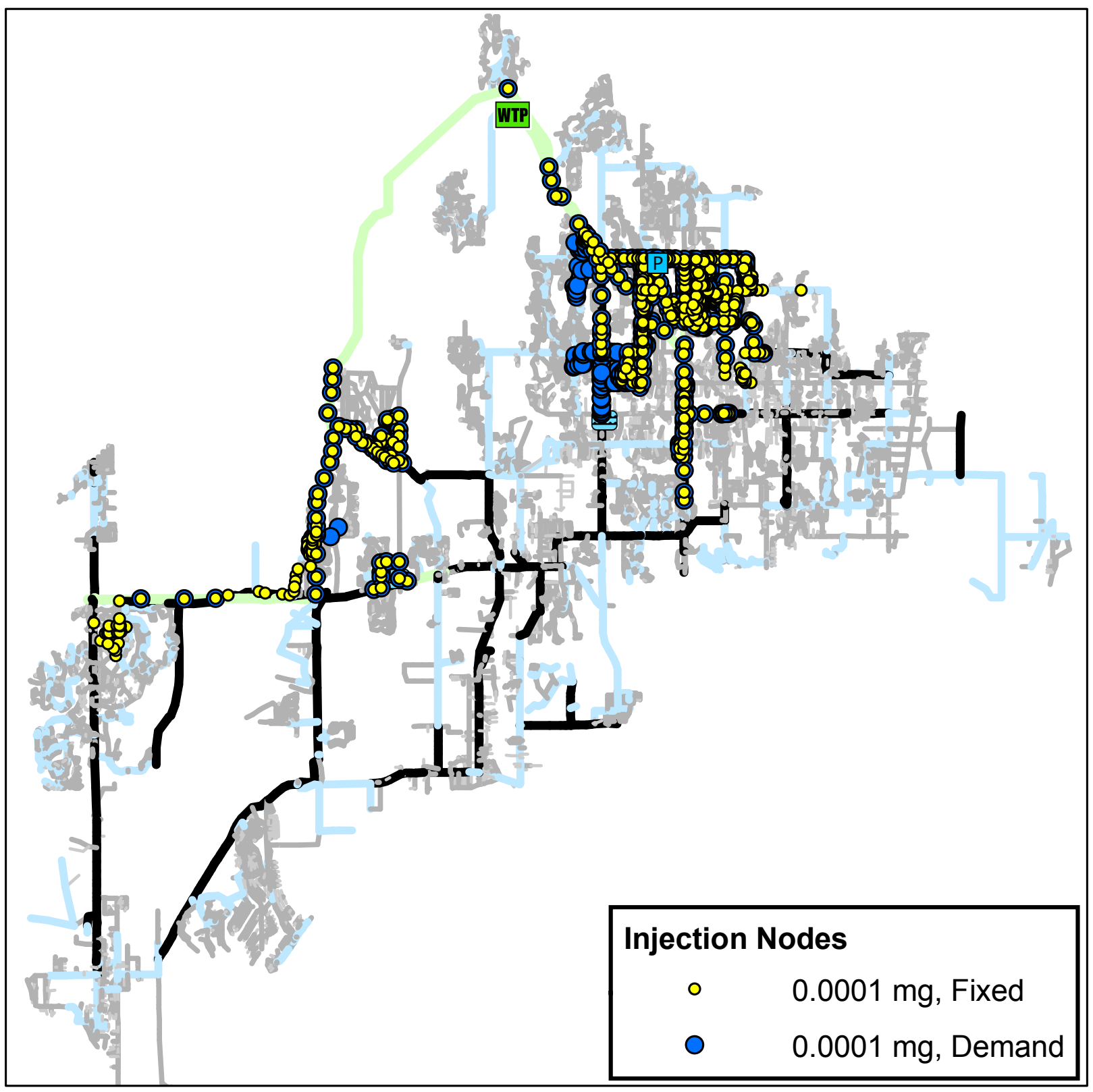

Figure 7.16. Network 6 showing locations of injection nodes associated with $95^{\text {th }}$ percentile or higher impacts at a dose level of $0.0001 \mathrm{mg}$ for the two population models. 


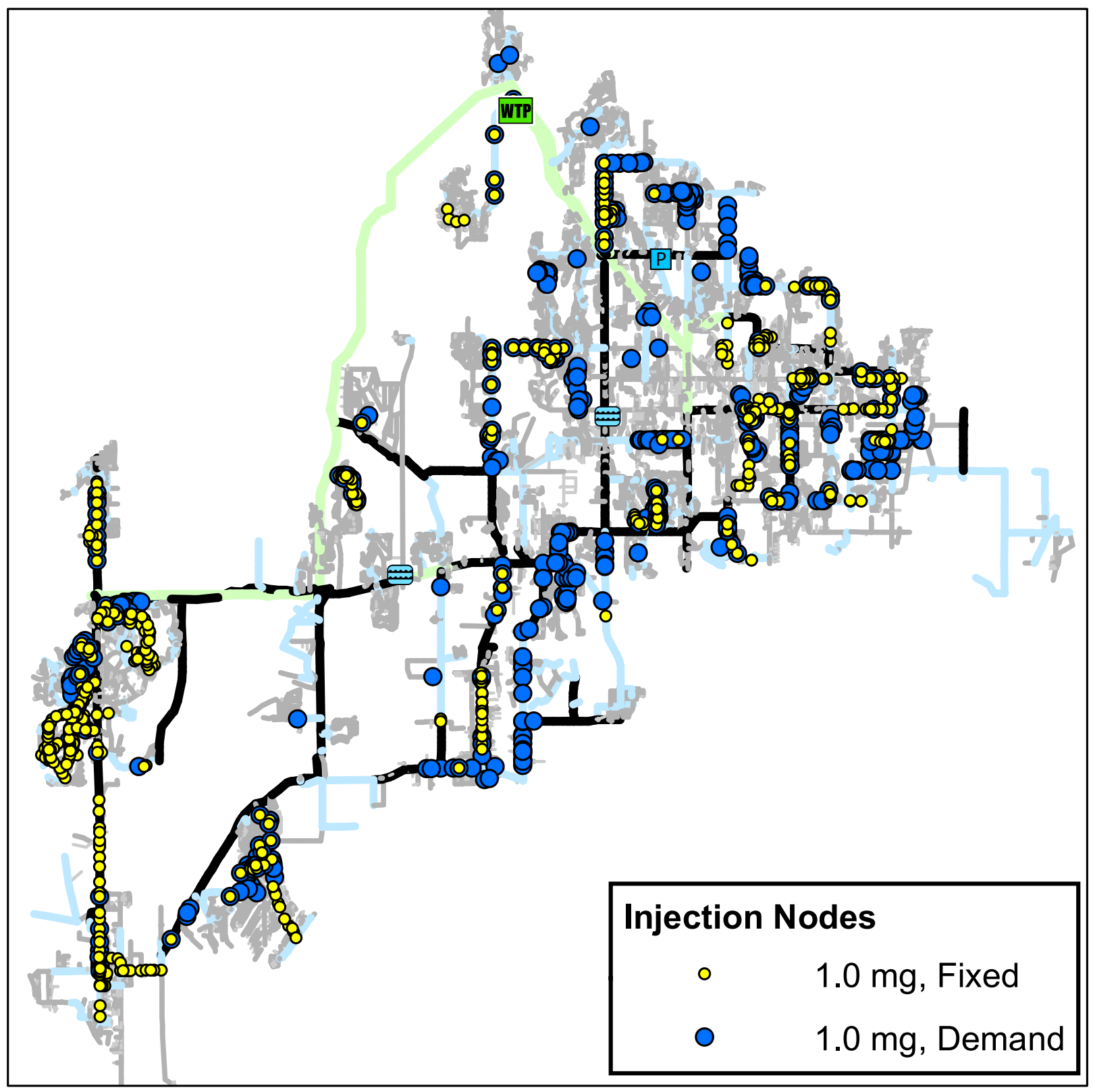

Figure 7.17. Network 6 showing locations of injection nodes associated with $95^{\text {th }}$ percentile or higher impacts at a dose level of $1.0 \mathrm{mg}$ for the two population models. 


\section{Section 8}

\section{Sensitivity of Impacts to Ingestion Model}

In this section we present our analysis of the sensitivity of impacts to the ingestion model used in estimating the impacts. The presentation follows that used for the other factors in the previous sections.

Figures 8.1 and 8.2 compare ranked impacts for Network 12 estimated using the two ingestion models (Models 1 and 2, described in Table 2.1). The deterministic model (Model 2) consistently yields the larger impacts. The ratio of impacts for this network tends to increase with dose level. The relative impacts are approximately four or less.

The sensitivity of ranked impacts to the ingestion model varies by network (Figures 8.3 and 8.4). For some networks sensitivity tends to increase with dose level except for the worst-case injection nodes $\left(100^{\text {th }}\right.$ percentile). For the majority of the networks the ratios of impacts are about four or less. For some networks the differences in results can be larger. (For comparison, absolute values for impacts for the networks obtained using Model 1 are provided in Figures 3.1 to 3.3.)

The distributions of impacts for Network 12 are most similar for the two ingestion models for the lowest dose level (Figures 8.5 and 8.6). There can be considerable differences in the upper tails of the distributions for higher dose levels.

When compared injection node by injection node, there can be a considerable difference in the impacts estimated for Network 12 using the two ingestion models, as illustrated in the plots in Figures 8.7 and 8.8. The scatter in the plots increases with dose level up to a dose level of $0.1 \mathrm{mg}$. The overlap of high percentile nodes for Network 12, illustrated by plots in Figures 8.9, 8.10, and 8.11 , decreases considerably with dose level, at least up to a dose level of $0.1 \mathrm{mg}$. At the $95^{\text {th }}$ percentile, the overlap at a dose level of $0.1 \mathrm{mg}$ is only about 0.1 (Figure 8.11).

The extent of overlap of high percentile injection nodes varies substantially across the networks (Figures 8.12 and 8.13). At the lowest dose level, the overlap is high for all impact levels, except for Network 7. Network 7 tends to have the lowest overlap of injection nodes for dose levels of $1.0 \mathrm{mg}$ or lower, but other networks can also have low overlap of high percentile nodes for the two different models. At higher dose levels, the overlap of high percentile nodes is low for all the networks.

Maps illustrating the overlap of high percentile injection nodes obtained using the two ingestion models are shown in Figures 8.14 through 8.17. Figures 8.14 and 8.15 provide results for Network 4 for dose levels of $0.0001 \mathrm{mg}$ and $1.0 \mathrm{mg}$, respectively and Figures 8.16 and 8.17 provide similar results for Network 6. As indicated in Figures 8.12 and 8.13, the overlaps are high at the lower dose level but low at the higher dose level. 
The magnitudes of ranked impacts can be sensitive to the ingestion model for some networks, particularly at higher dose levels. However, the more important sensitivity to these models involves the impacts associated with individual injection nodes. The overlap of the injection nodes associated with the highest impacts can be low for some networks for the two ingestion models even for relatively low dose levels. There is considerable variability from network to network. 


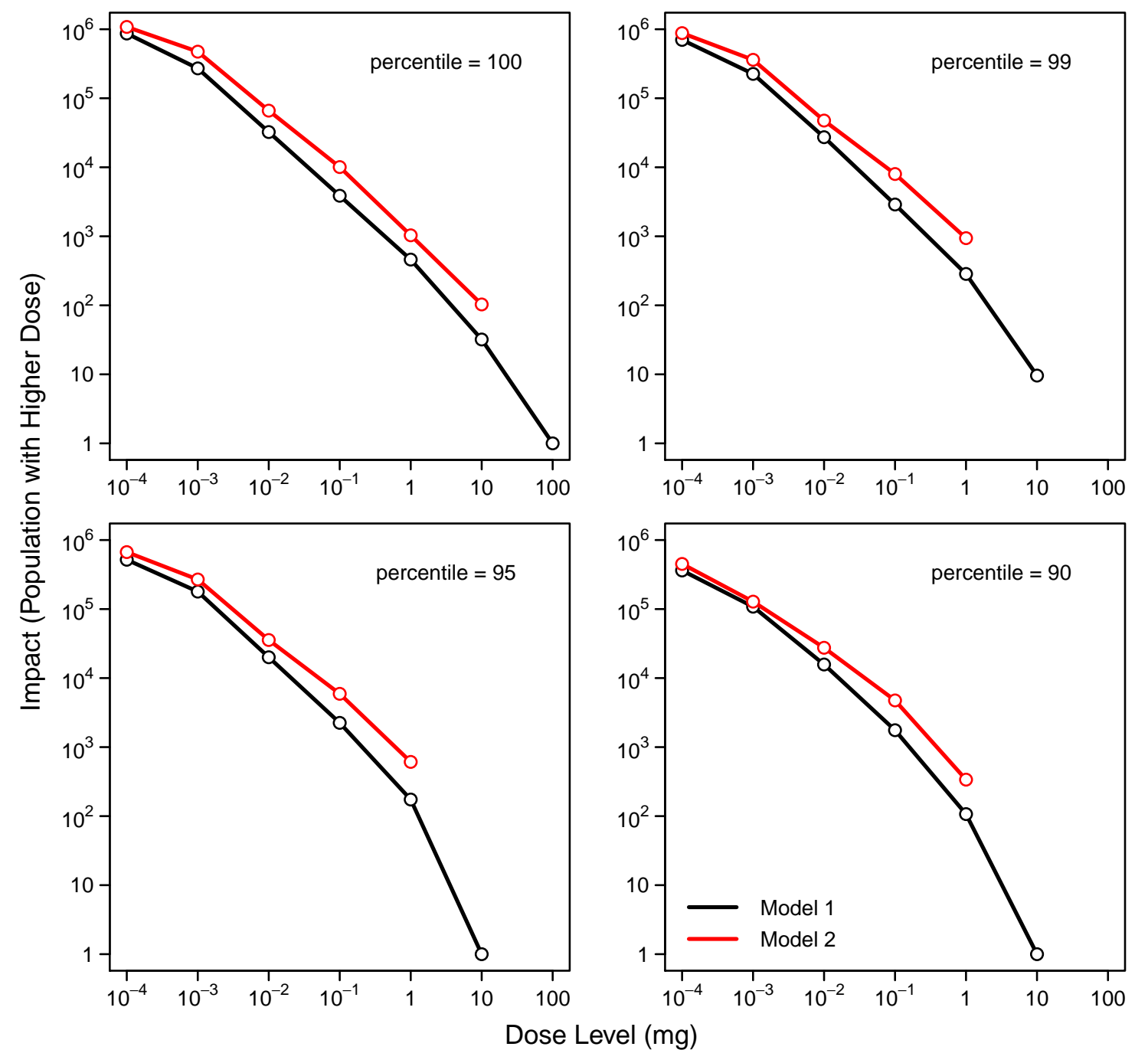

Figure 8.1. Impacts associated with different ingestion models for Network 12 as a function of dose level, for the $90^{\text {th }}, 95^{\text {th }}, 99^{\text {th }}$, and $100^{\text {th }}$ percentile injection nodes. 


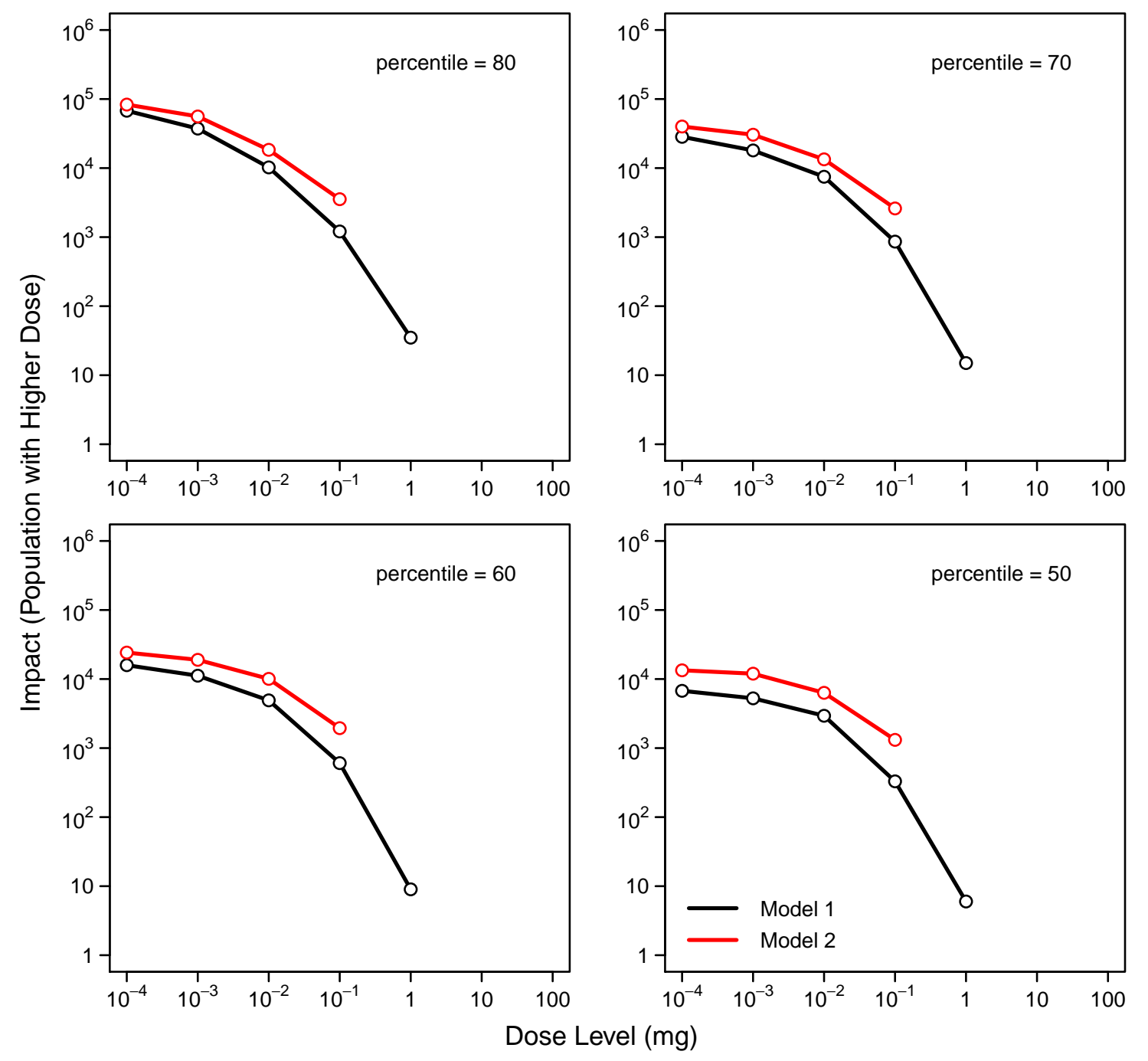

Figure 8.2. Impacts associated with different ingestion models for Network 12 as a function of dose level, for $50^{\text {th }}, 60^{\text {th }}, 7^{\text {th }}$, and $80^{\text {th }}$ percentile injection nodes. 


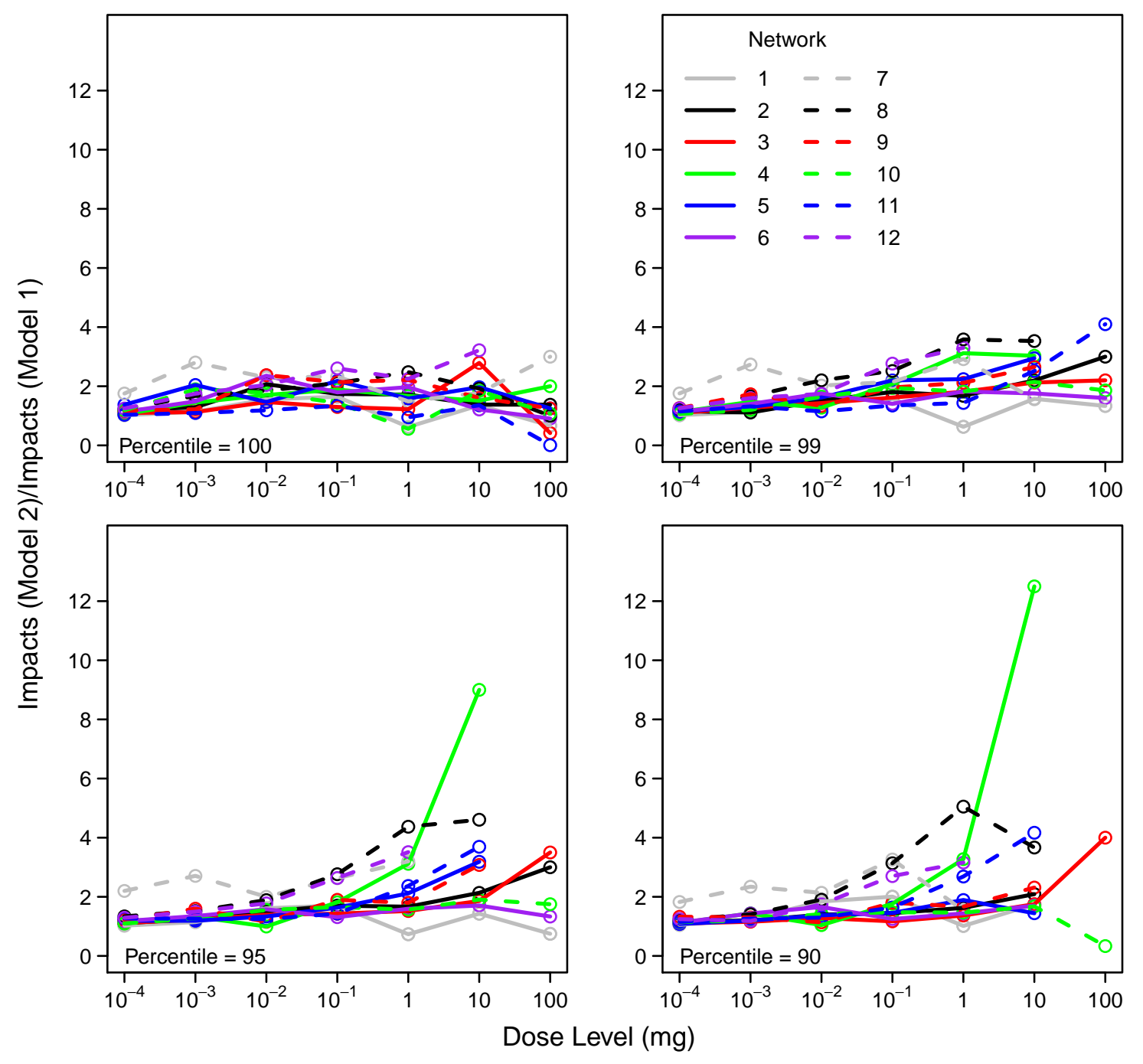

Figure 8.3. Relative magnitudes of the impacts for the networks obtained with different ingestion models as a function of dose level, for the $90^{\text {th }}, 95^{\text {th }}, 99^{\text {th }}$, and $100^{\text {th }}$ percentile injection nodes. 


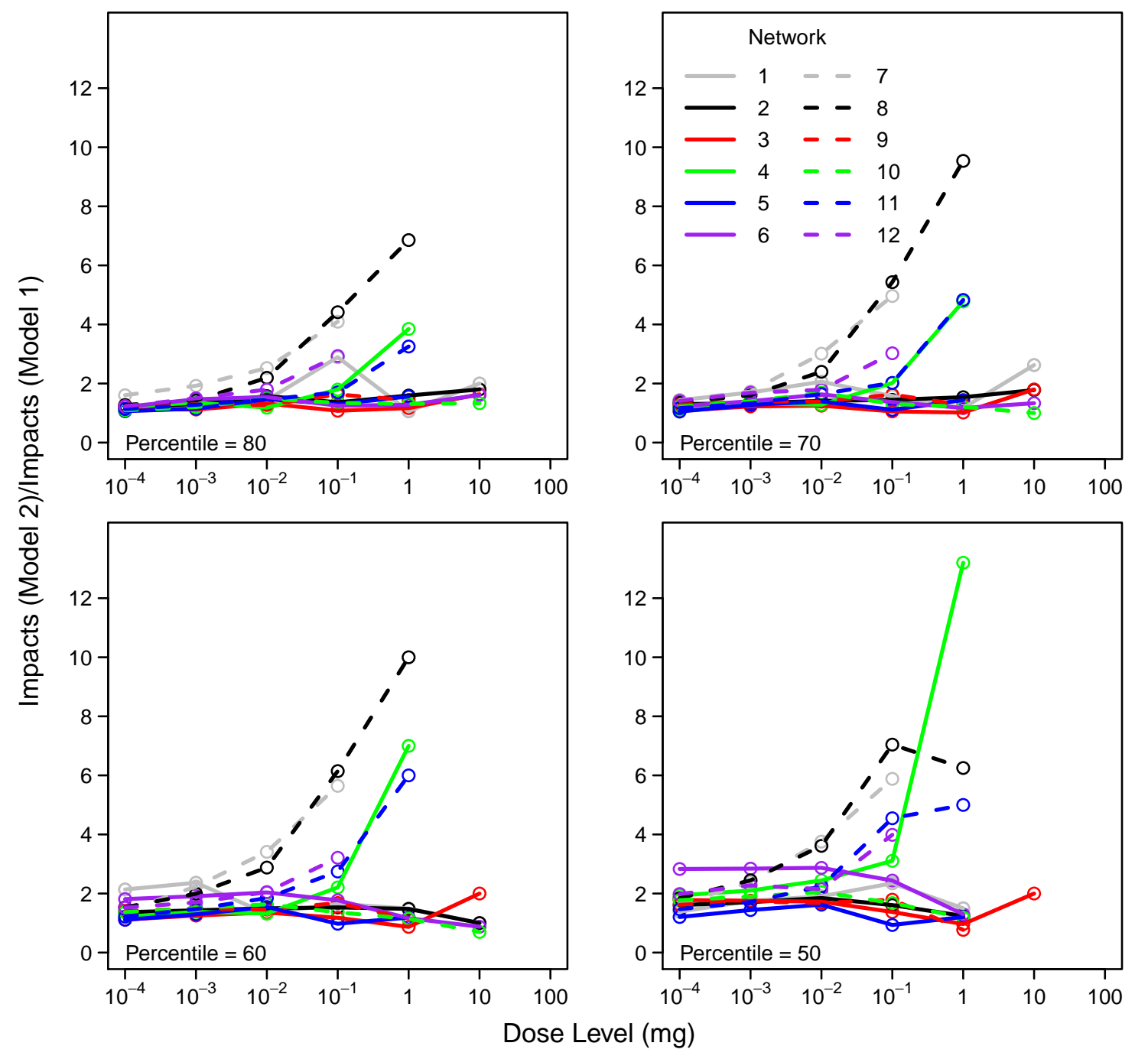

Figure 8.4. Relative magnitudes of the impacts for the networks obtained with different ingestion models as a function of dose level, for $50^{\text {th }}, 60^{\text {th }}, 70^{\text {th }}$, and $80^{\text {th }}$ percentile injection nodes. 


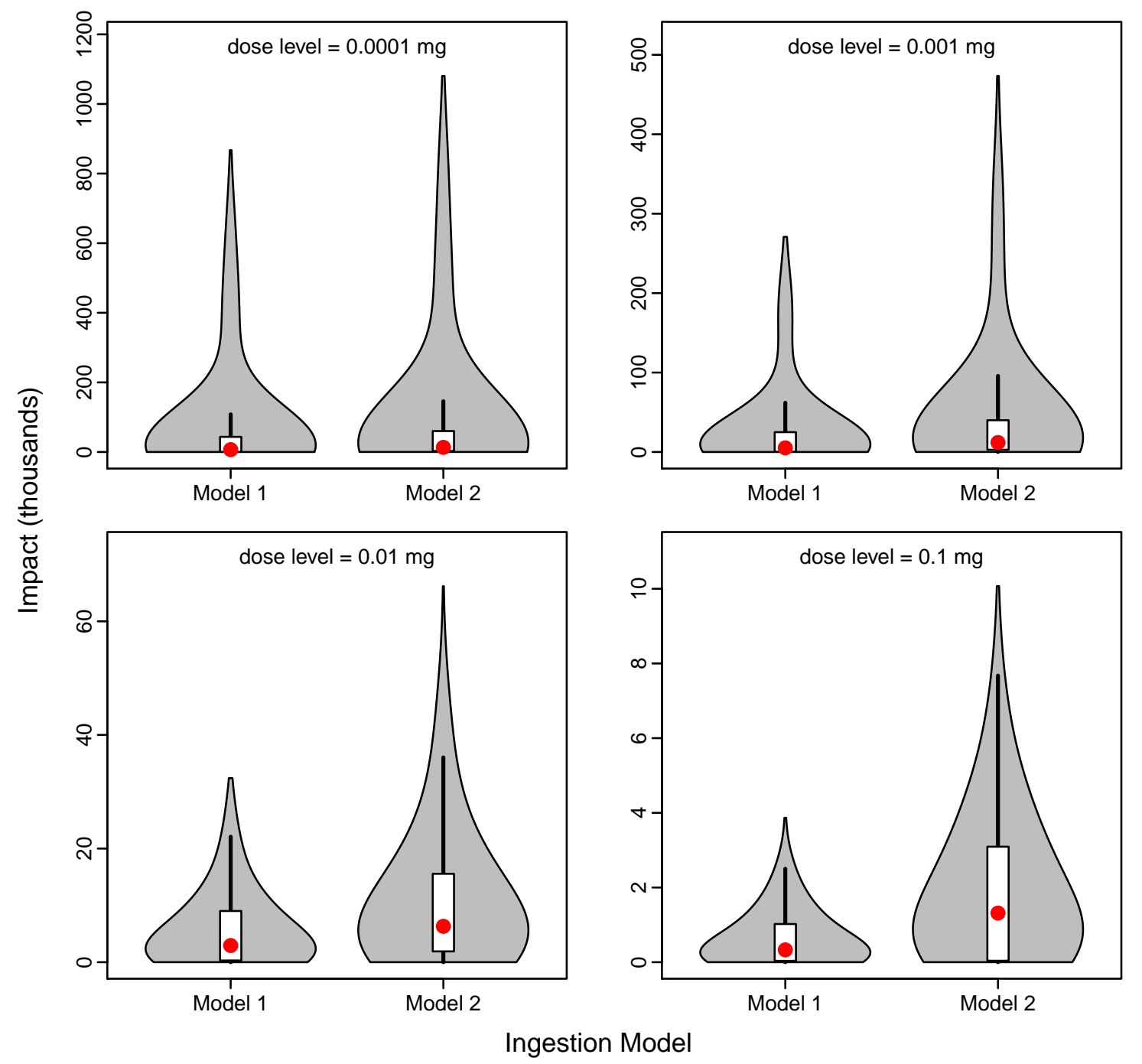

Figure 8.5. Distributions of impacts for Network 12 across all injection nodes for different ingestion models, for dose levels of $0.0001,0.001,0.01$, and $0.1 \mathrm{mg}$. 


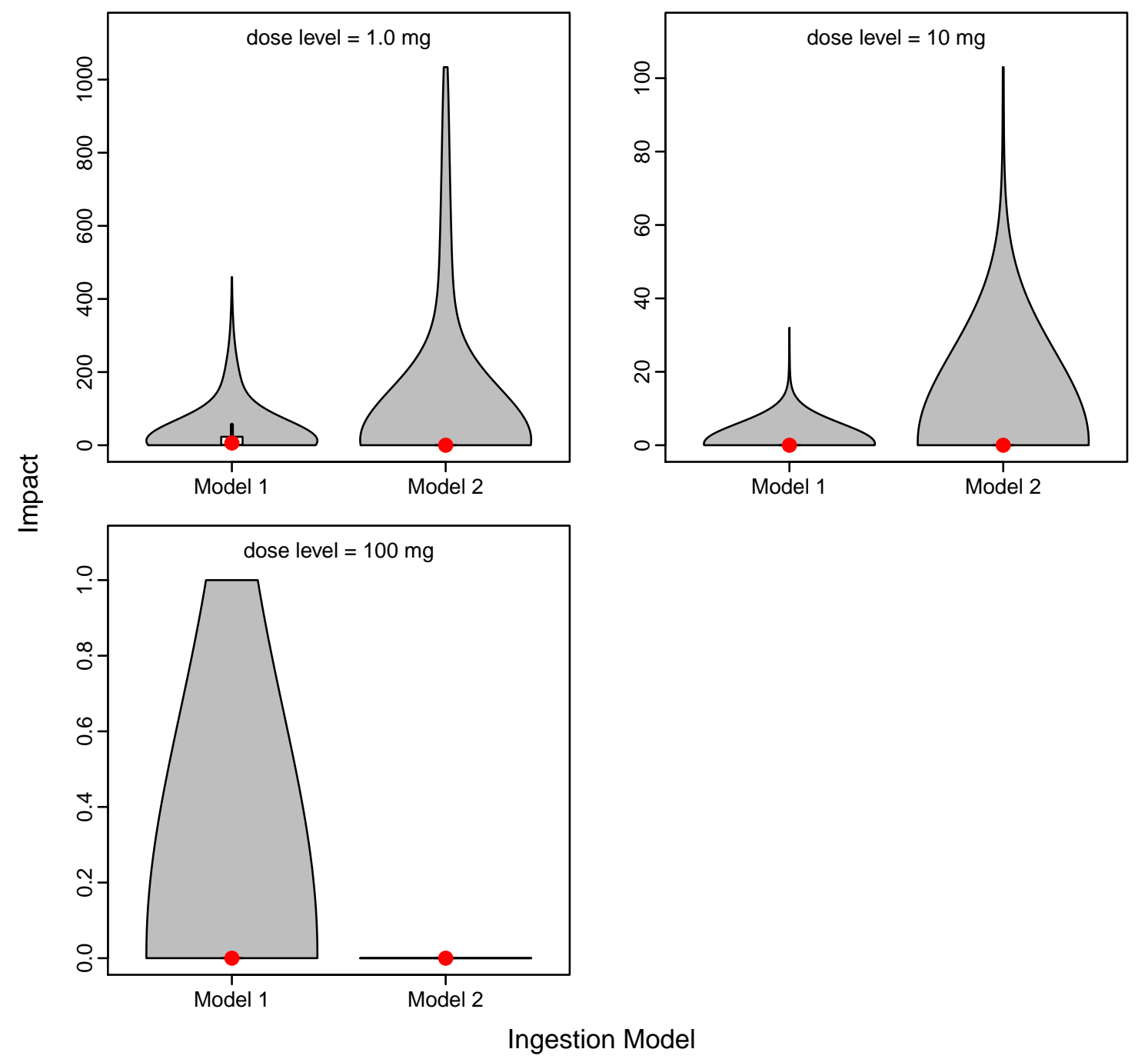

Figure 8.6. Distributions of impacts for Network 12 across all injection nodes for different ingestion models, for dose levels of 1,10 , and $100 \mathrm{mg}$. 


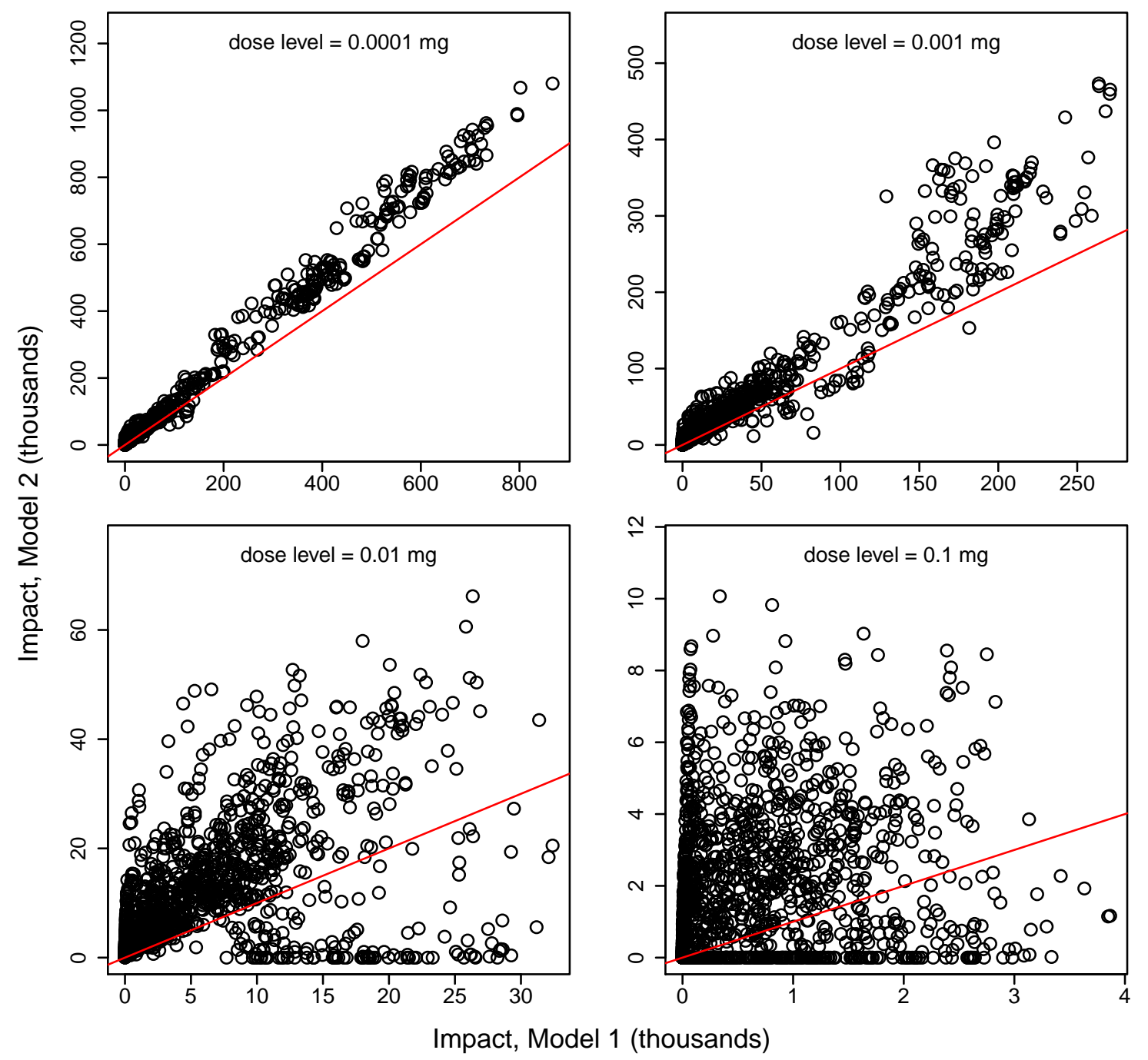

Figure 8.7. Impacts for Network 12 obtained with different ingestion models, for dose levels of $0.0001,0.001,0.01$, and $0.1 \mathrm{mg}$. The red lines have a slope of 1 . Each point corresponds to one injection node. 


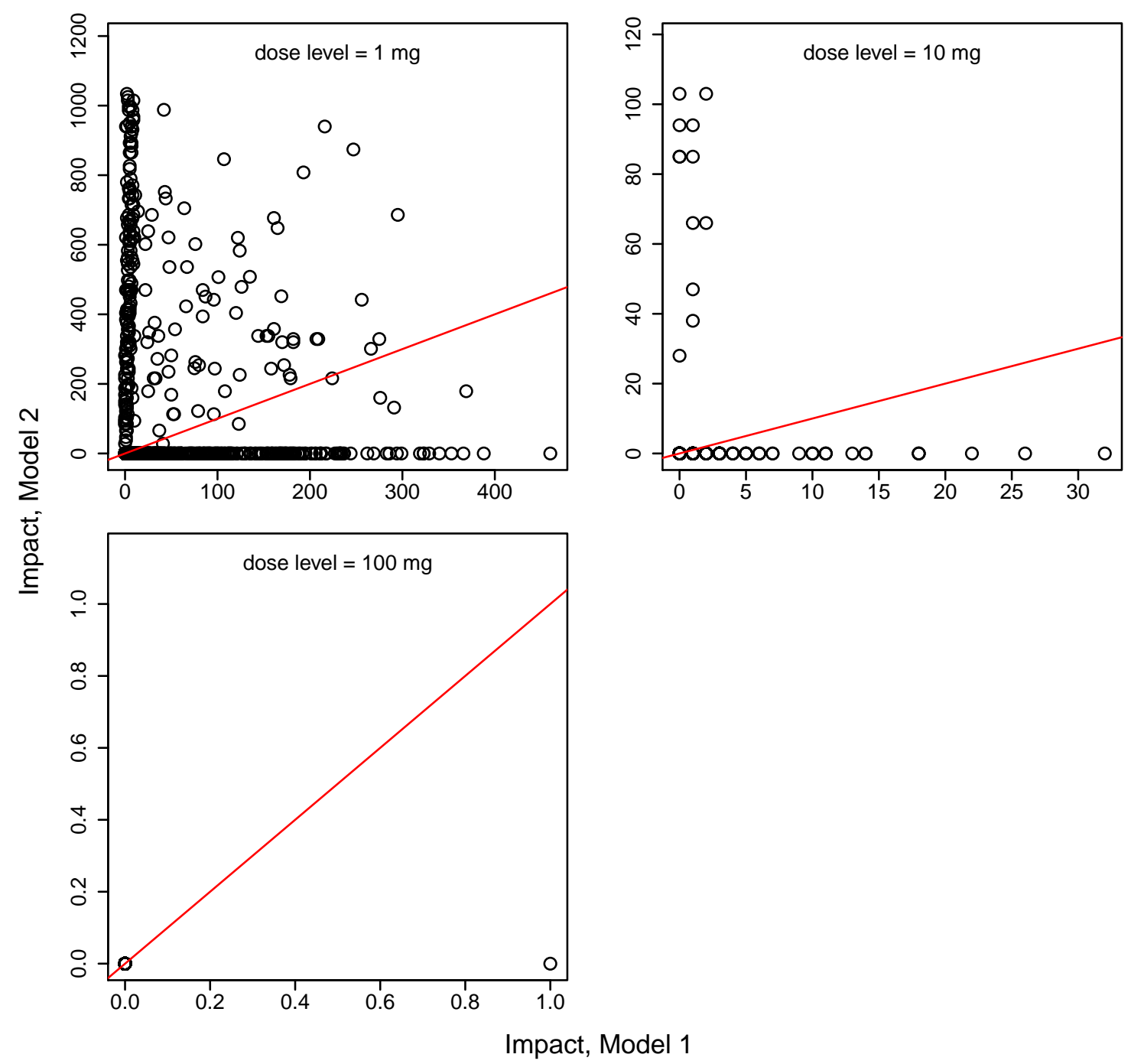

Figure 8.8. Impacts for Network 12 obtained with different ingestion models, for dose levels of 1,10 , and $100 \mathrm{mg}$. The red lines have a slope of 1 . Each point corresponds to one injection node. 


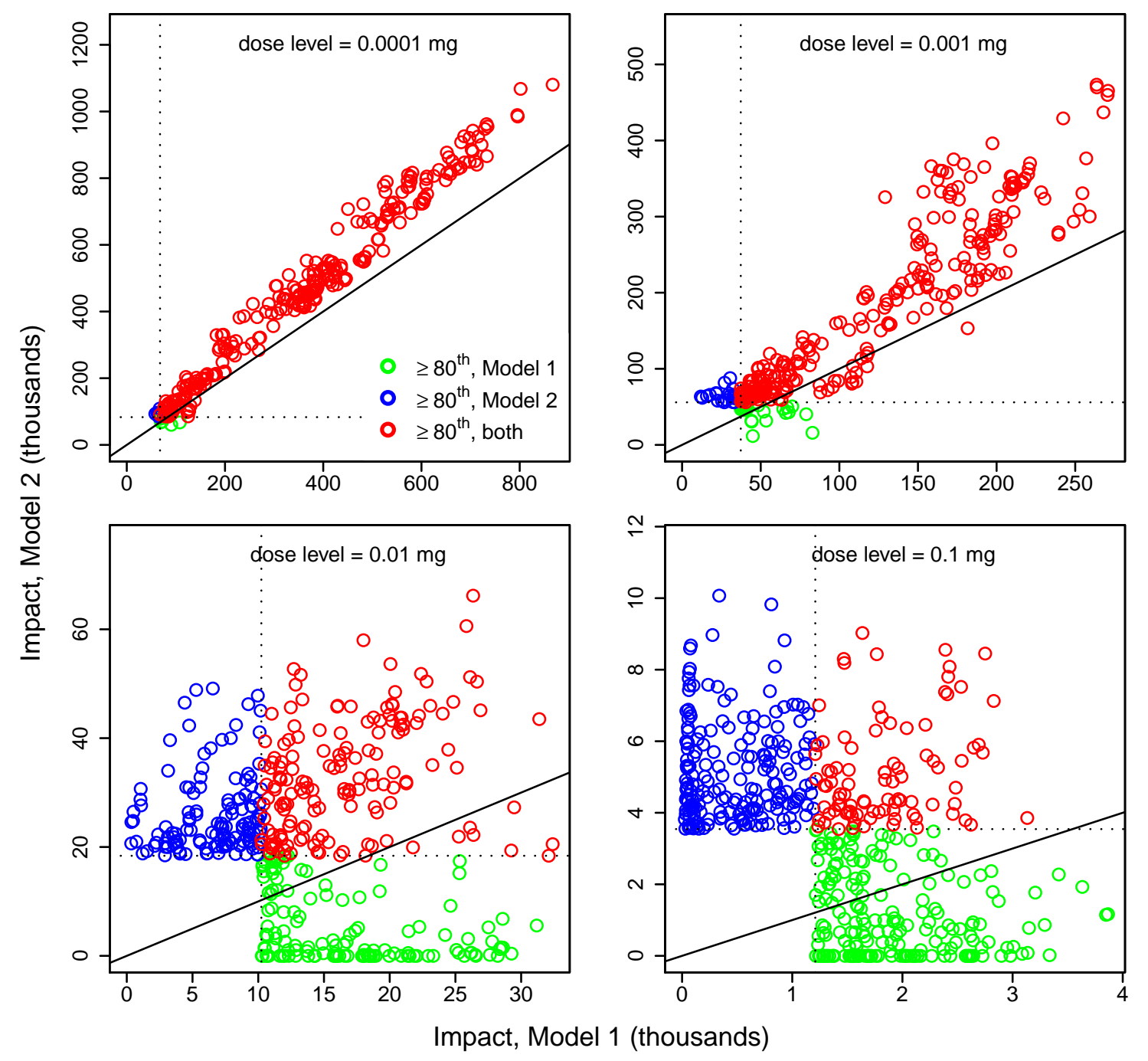

Figure 8.9. Impacts for Network 12 that are at or above the $80^{\text {th }}$ percentile level obtained with different ingestion models, for dose levels of $0.0001,0.001,0.01$, and $0.1 \mathrm{mg}$. The dotted lines indicate $80^{\text {th }}$ percentile impacts. The black lines have a slope of 1 . Each point corresponds to one injection node. 


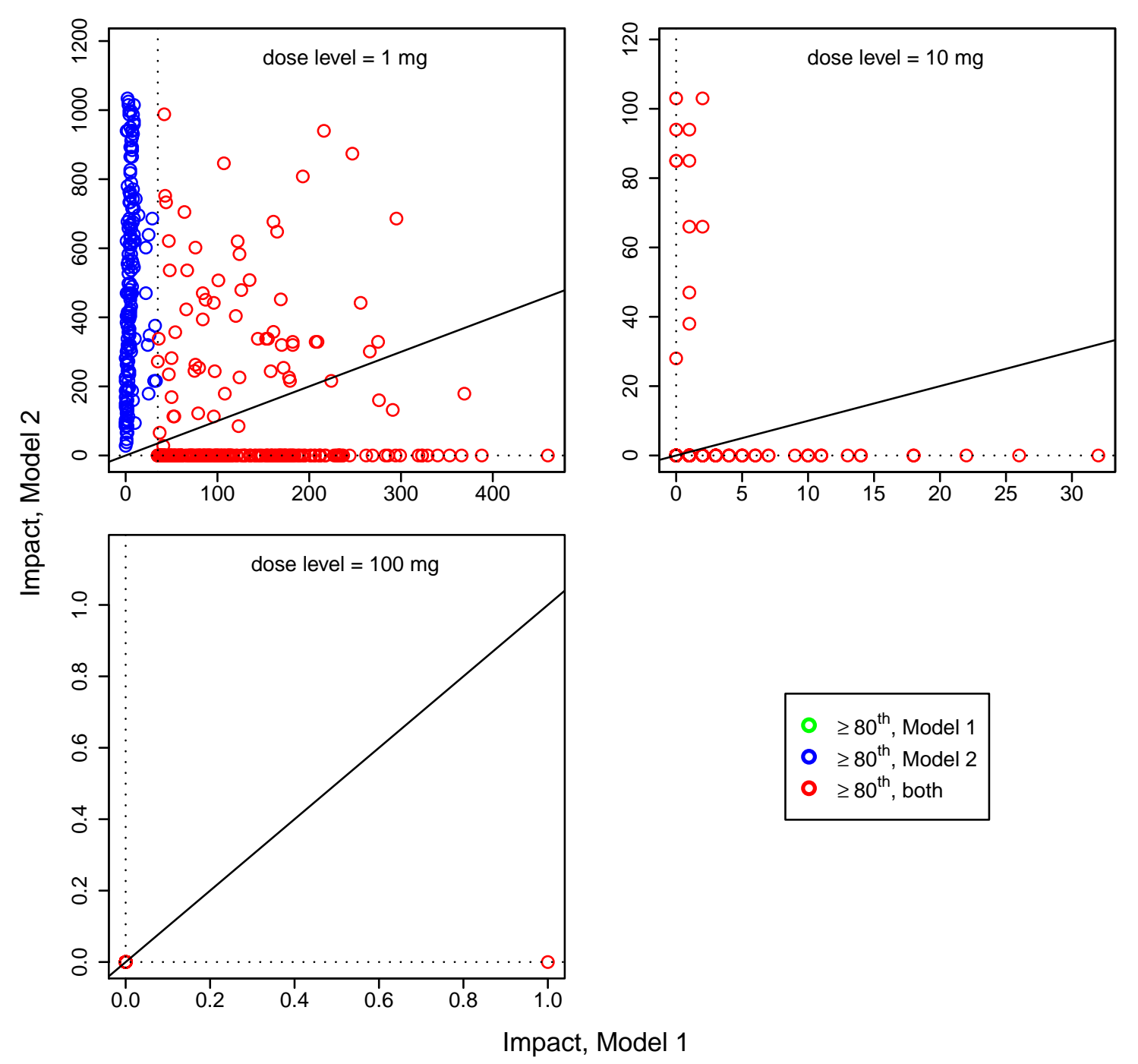

Figure 8.10. Impacts for Network 12 that are at or above the $80^{\text {th }}$ percentile level obtained with different ingestion models, for dose levels of 1,10 , and $100 \mathrm{mg}$. The dotted lines indicate $80^{\text {th }}$ percentile impacts. The black lines have a slope of 1 . Each point corresponds to one injection node. 


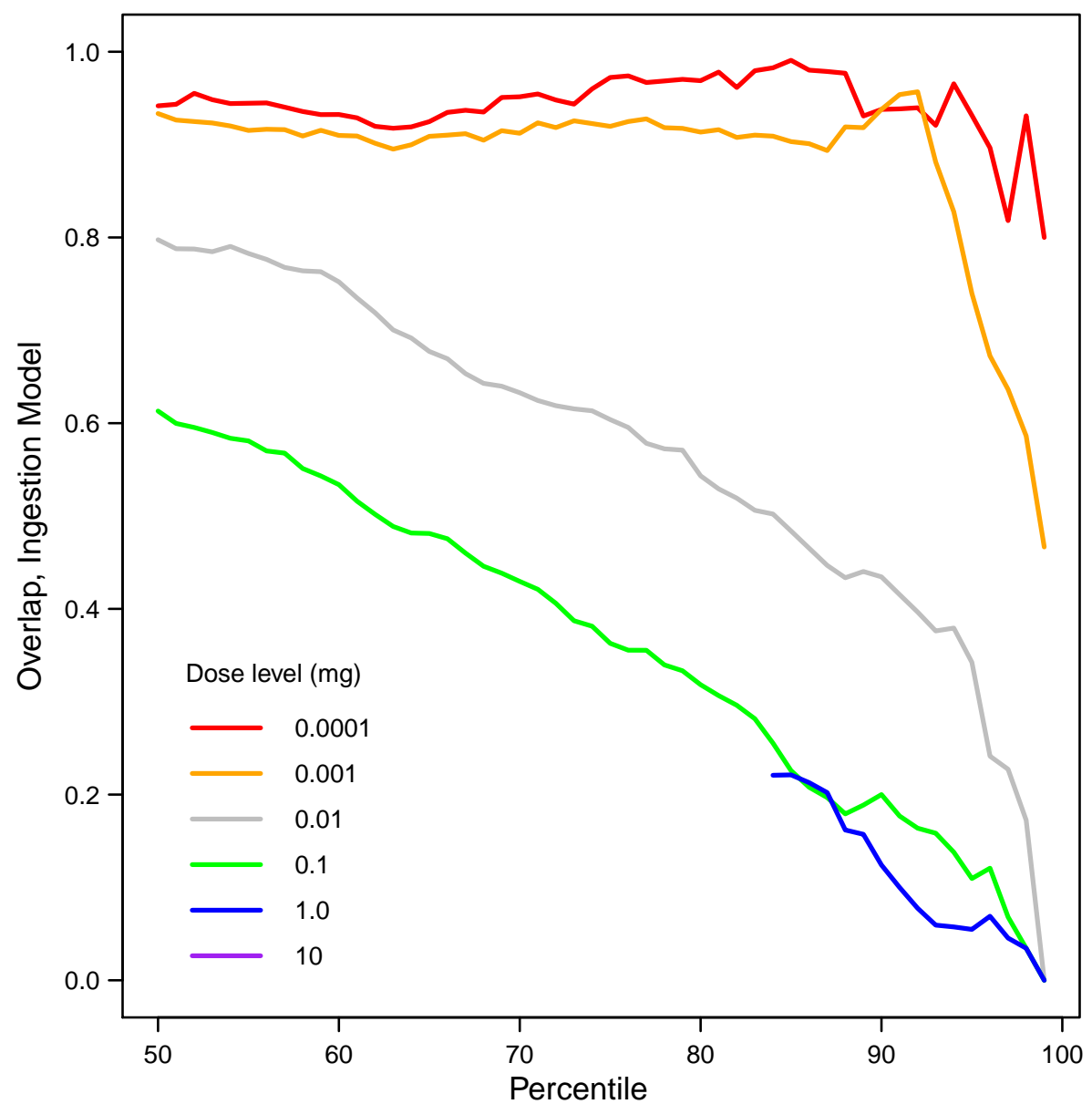

Figure 8.11. Overlap of high percentile injection nodes for Network 12 for different ingestion models for different dose levels. 

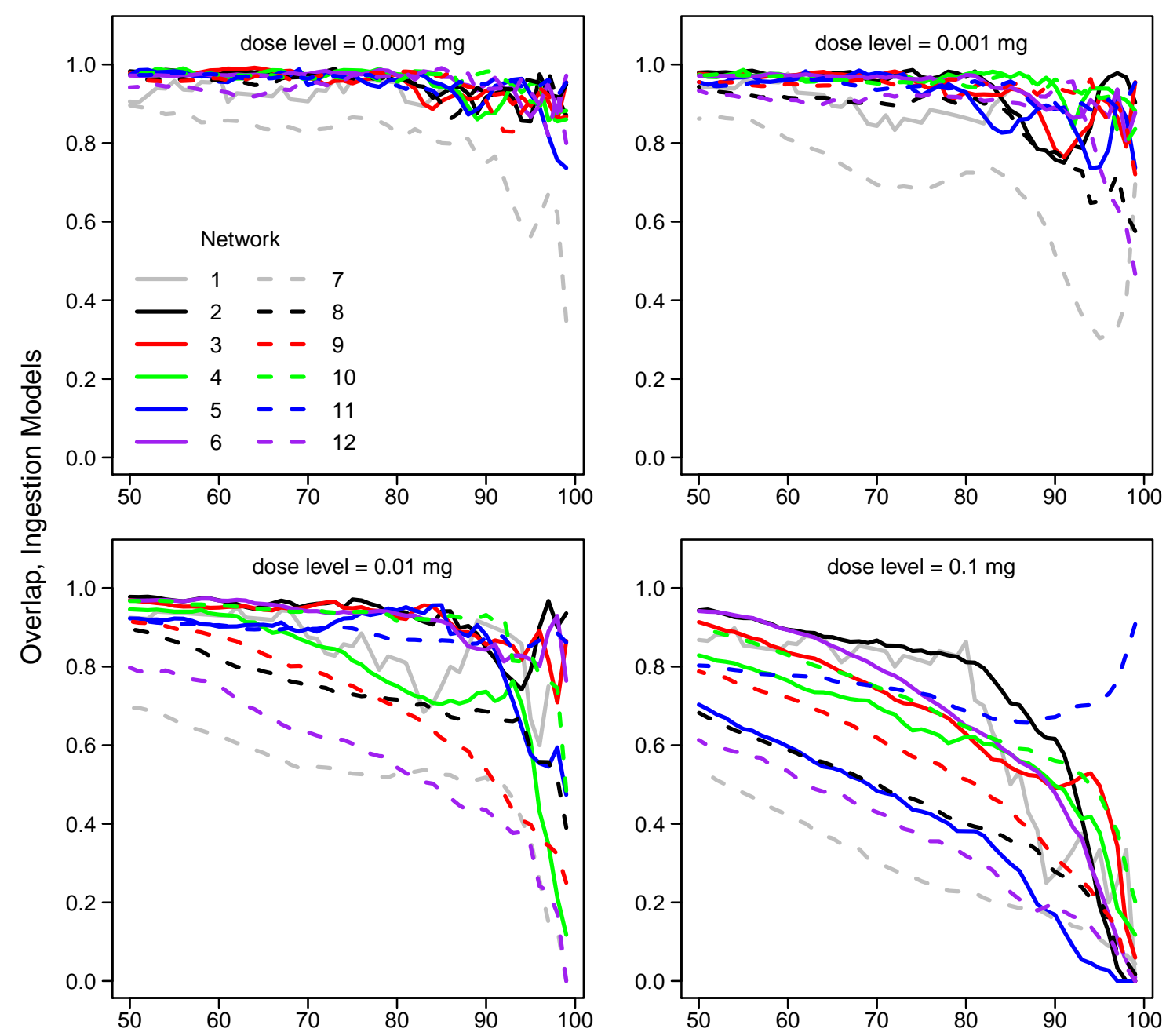

Percentile

Figure 8.12. Overlap of high percentile injection nodes for the networks for the two ingestion models, for dose levels of $0.0001,0.001,0.01$, and $0.1 \mathrm{mg}$. 


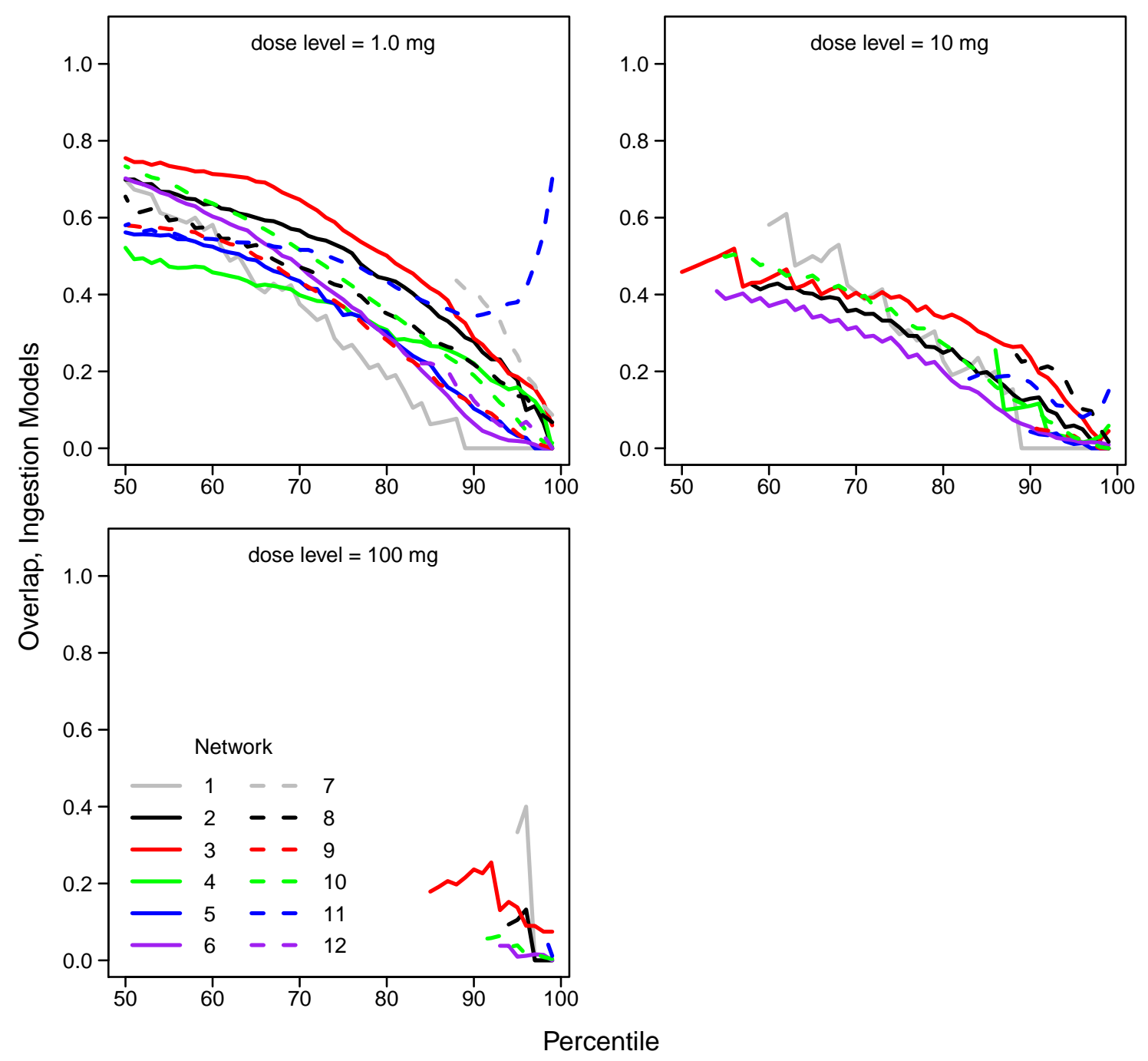

Figure 8.13. Overlap of high percentile injection nodes for the networks for the two ingestion models, for dose levels of 1,10 , and $100 \mathrm{mg}$. 


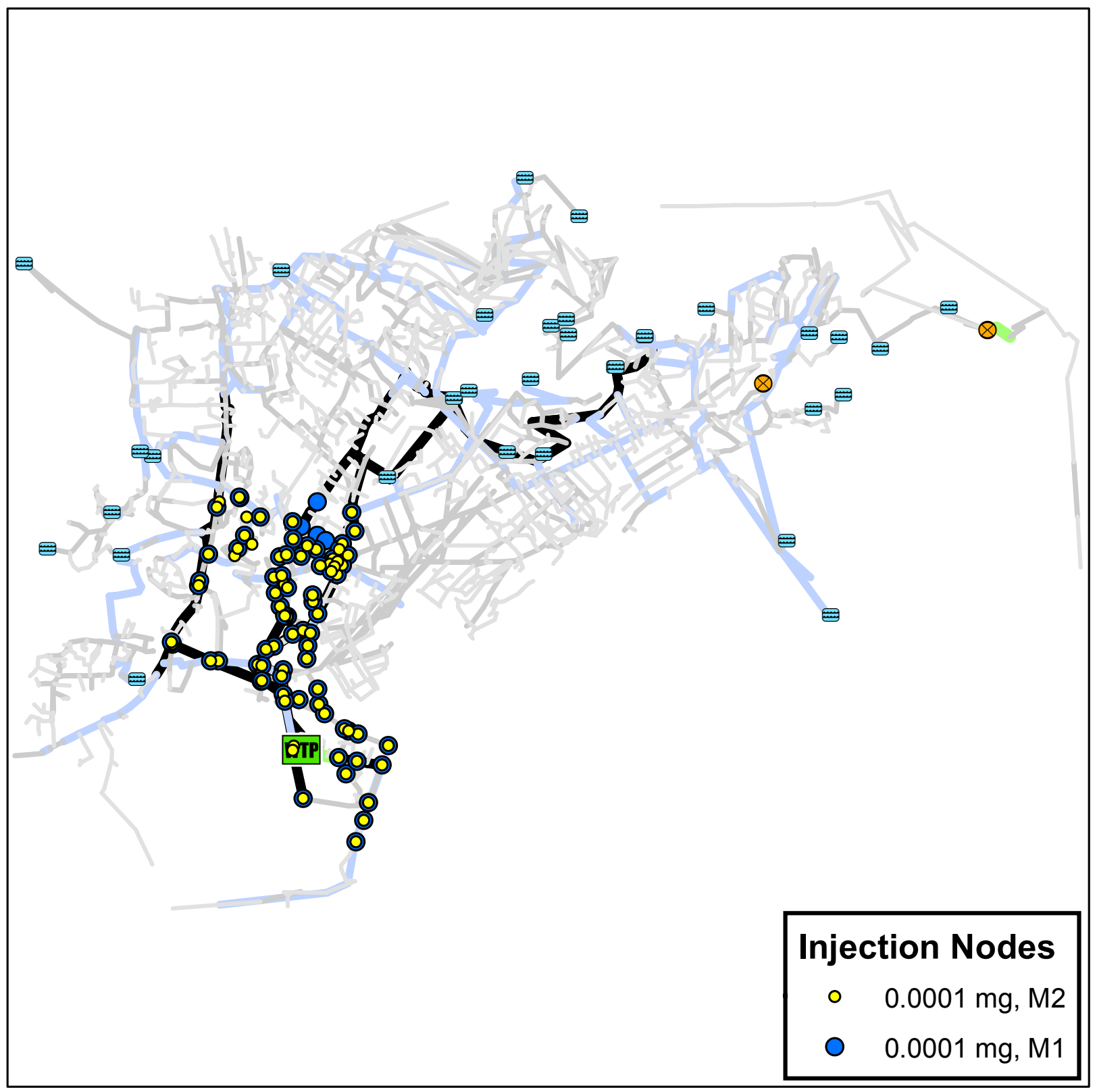

Figure 8.14. Network 4 showing locations of injection nodes associated with $95^{\text {th }}$ percentile or higher impacts at a dose level of $0.0001 \mathrm{mg}$ for the two ingestion models. M1 is Model 1 and M2 is Model 2. 


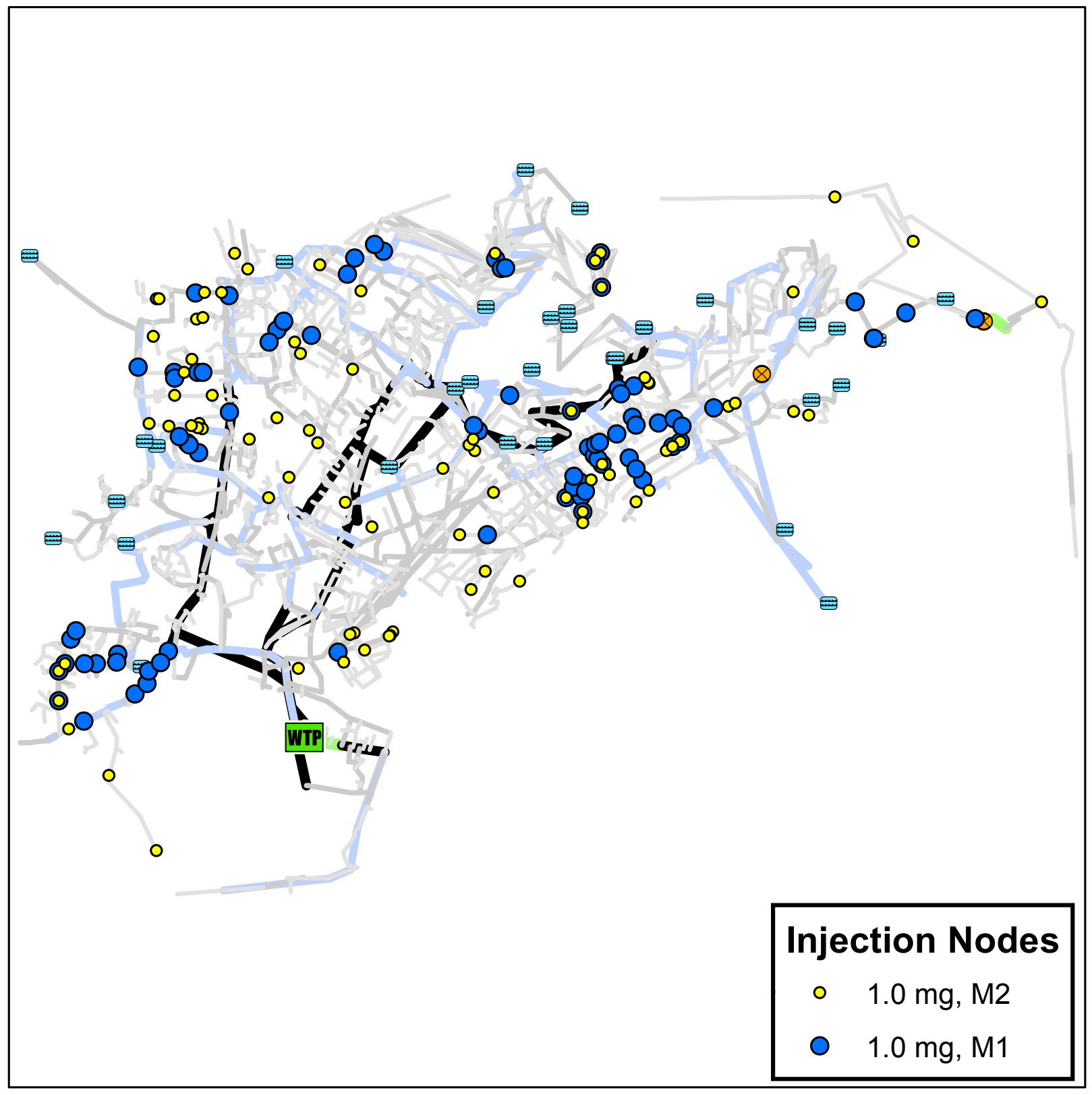

Figure 8.15. Network 4 showing locations of injection nodes associated with $95^{\text {th }}$ percentile or higher impacts at a dose level of $1.0 \mathrm{mg}$ for the two ingestion models. M1 is Model 1 and M2 is Model 2. 


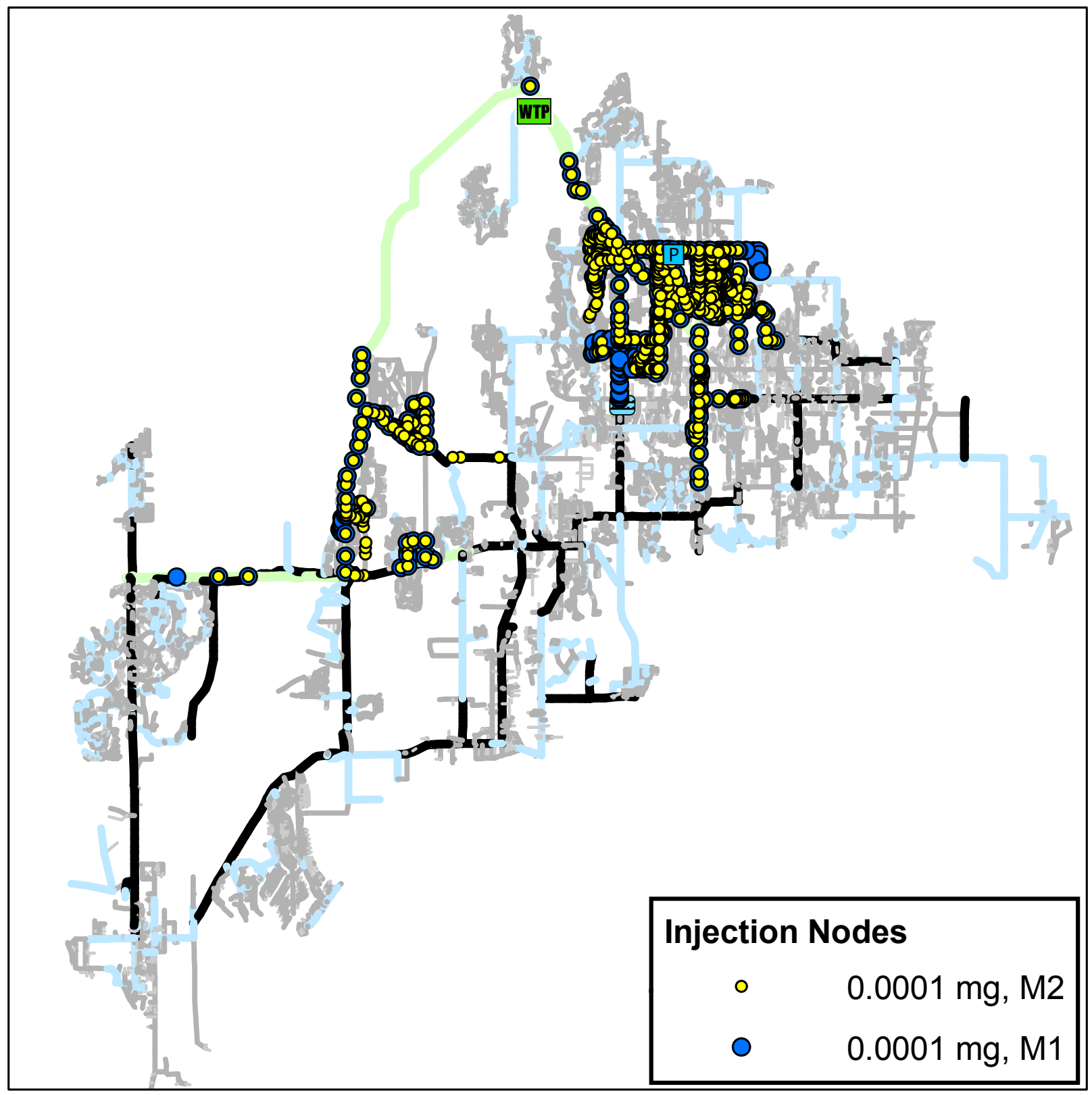

Figure 8.16. Network 6 showing locations of injection nodes associated with $95^{\text {th }}$ percentile or higher impacts at a dose level of $0.0001 \mathrm{mg}$ for the two ingestion models. M1 is Model 1 and M2 is Model 2. 


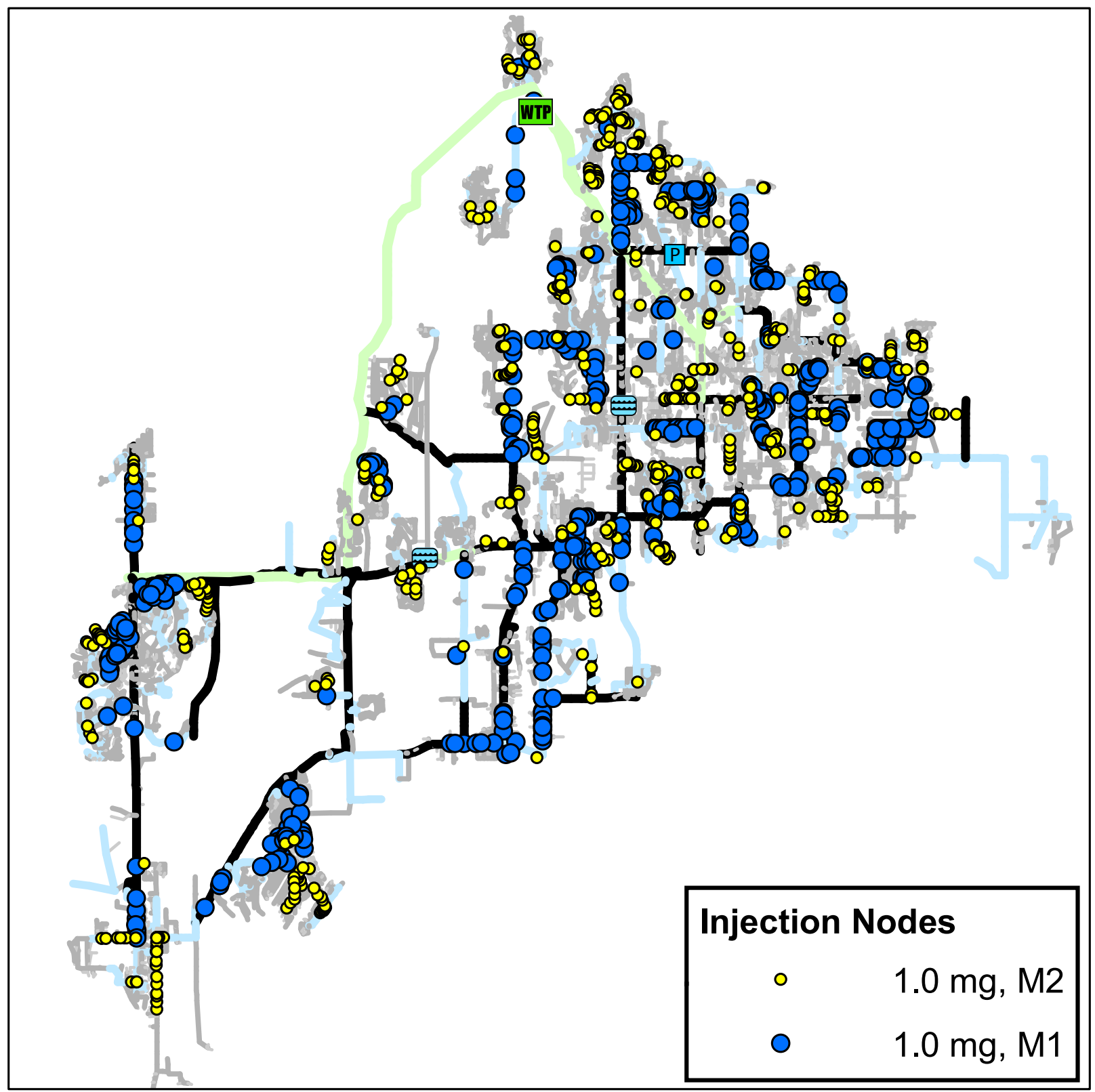

Figure 8.17. Network 6 showing locations of injection nodes associated with $95^{\text {th }}$ percentile or higher impacts at a dose level of $1.0 \mathrm{mg}$ for the two ingestion models. M1 is Model 1 and M2 is Model 2. 


\section{Section 9}

\section{Sensitivity of Impacts to Combinations of Factors}

The preceding analysis examined the influence of changes in one factor while all others were held constant. However, in reality, the various factors do not affect estimated impacts in isolation. It is therefore necessary to examine the importance of changes in the factors when variation in all factors is assumed.

As discussed in Section 2, we examined the sensitivity of impacts to combinations of factors. We consider four injection times (0:00, 6:00, 12:00, and 18:00), two injection durations (0 and $24 \mathrm{~h}$ ), two injection masses (1X and 10X), and two population distributions (average population value at each node and demand-based population at each node), yielding a total of 32 combinations of factors. Considering the ingestion model in addition to these four factors would have doubled the number of required simulations and therefore was not pursued.

Figures 9.1 through 9.4 provide histograms of the impacts obtained for the 32 cases (combinations of factors) for Network 2. Results are shown for four dose levels $(0.0001,0.01,1$, and $100 \mathrm{mg})$ and for four impact levels $\left(100^{\text {th }}, 90^{\text {th }}, 70^{\text {th }}\right.$, and $50^{\text {th }}$ percentiles $)$. Note that impacts in Figures 9.1 and 9.3 are in units of thousands. Using the $100^{\text {th }}$ percentile plot for a dose level of $0.0001 \mathrm{mg}$ in Figure 9.1 as an example, there are six cases that have impacts that lie in the bin centered on 75,000 impacts. The bin has a width of 2,000 impacts. This means that six out of the 32 cases have their largest impacts in the range from 74,000 to 76,000. Similarly, one case out of the 32 has its $70^{\text {th }}$ percentile impacts for a dose level of $0.0001 \mathrm{mg}$ in the range from 3,000 to 3,200 .

At the lowest dose level, the magnitudes of the impacts for the various cases are similar (there is only a small change in their relative values), particularly at the higher percentiles. However, at the higher dose levels, the relative magnitudes of the impacts can vary substantially across the various cases. Changes in the various factors have a much larger influence on impacts at the higher dose levels than at the lower dose levels. The histograms in Figures 9.1 through 9.4 demonstrate that changes in the factors can significantly influence impacts, but they do not tell us which factors have the most important influence when all factors are considered together. That information can be obtained from Figures 9.5 through 9.20.

Figures 9.5 through 9.20 provide the same histograms as shown in Figures 9.1 through 9.4. However, they are now color coded to show the relationship of impacts to injection time (Figures 9.5 through 9.8), injection duration (Figures 9.9 through 9.12), injection mass (Figures 9.13 
through 9.16), and population distribution (Figures 9.17 through 9.20). In the histograms, a particular color corresponds to a particular value of a factor. For example, in Figure 9.5 blue corresponds to an injection time of 12:00. If colors are distributed more or less uniformly across a histogram, then variations in the factor are not having much influence on the magnitudes of the impacts. If patterns are present in the distribution of the colors then impacts are being influenced differently by different values of the factor. For example, in Figure 9.9, at a dose level of $0.0001 \mathrm{mg}$, there is strong division between the distribution of impacts associated with an injection duration of $1 \mathrm{~h}$ and that of an injection duration of $24 \mathrm{~h}$. The impacts associated with the latter are larger than those associated with the former, independent of all the other factors. However, at a dose level of $100 \mathrm{mg}$ (Figure 9.12) the pattern disappears and no simple relationship between injection time and impact is evident. Figures 9.13 throught 9.16 show similar results for injection mass, except that now the simple relationship between the factor and impacts occurs for higher dose levels (high injection mass results in high impacts) but disappears at the lowest dose level. As would be expected, at higher dose levels the higher impacts generally are associated with the $10 \mathrm{X}$ injection mass. Increasing the injection mass by a factor of ten generally results in increased impacts, independent of any other factor. However, at the lowest dose level the relationship between injection mass and impacts is less clear: impacts are not strongly related to injection mass at this dose level. No simple, consistent patterns are apparent for injection time (Figures 9.5 through 9.8) or the population model (Figure 9.17 through 9.20).

Overall, when all the factors are considered together on the basis of ranked impacts, impacts are most sensitive to injection mass and injection duration, but the degree of sensitivity depends on dose level. No significant relationship exists between impact level and injection time (Figures 9.5 through 9.8). Finally, impacts are not strongly related to the distribution used for population (Figures 9.17 through 9.20).

When considered on the basis of individual injection nodes, the degree of consistency with which particular nodes remain high impact injection nodes across the combinations of factors depends on the dose level and impact level. The overlap of nodes across the 32 combinations of factors is shown in Figures 9.21 to 9.23 for Network 2 for different dose levels for $75^{\text {th }}, 95^{\text {th }}$, and $99^{\text {th }}$ and higher percentile impacts. The horizontal axis in the plots gives a listing of individual injection nodes. It could be labeled with the identification of the individual nodes. However, given space constraints in the plots, the nodes are simply labeled 1, 2, 3 etc., after sorting the nodes by the number of times the same node is a particular percentile or higher node for the 32 cases. If the same nodes were the high percentile injection nodes for all combinations of factors, the plots would show a constant count of 32 and a number of nodes equal to about 750, 150, and 30 for the case of $75^{\text {th }}, 95^{\text {th }}$, and $99^{\text {th }}$ and higher percentiles, respectively. (The number of nodes would be equal to about 3000 times the fraction of nodes at or above the given percentile, e.g., 0.25 for the $75^{\text {th }}$ percentile.) The dashed red lines show what the plots would look like if the same nodes were the high percentile nodes for all cases. The figures show that overlap is highest for the low dose levels and lower impact levels. For example, for a dose level of $0.0001 \mathrm{mg}$, the same 600 -plus nodes (out of a possible 750 or so) are $75^{\text {th }}$ percentile and higher impact nodes for all 32 combinations of factors (Figure 9.21). However, for a dose level of $100 \mathrm{mg}$ and the $99^{\text {th }}$ and higher percentiles (Figure 9.23), at most only about 10 combinations of factors share a common high impact node, out of a possible 32. In summary, the plots show that the consistency with which particular nodes remain high impact nodes is low for high dose levels, but can be high for low dose levels, as is shown for the $75^{\text {th }}$ percentile case in Figure 9.21. 


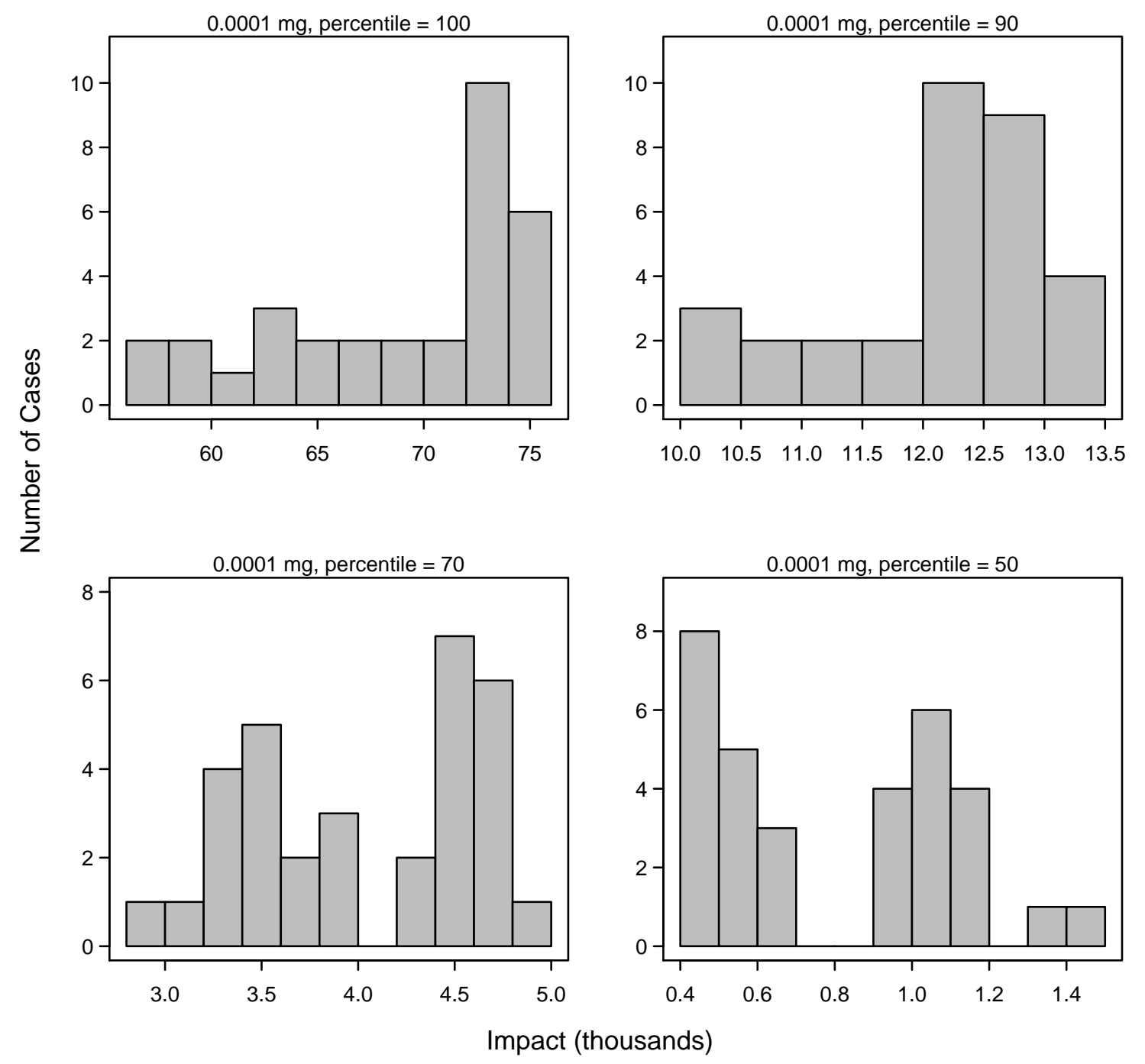

Figure 9.1. Histograms of impacts for the 32 combinations of factors for Network 2 for a dose level of $0.0001 \mathrm{mg}$ and different impact levels. The number of cases on the vertical axis is the number of combinations of factors, out of a total of 32, that are in a particular bin in a histogram. 

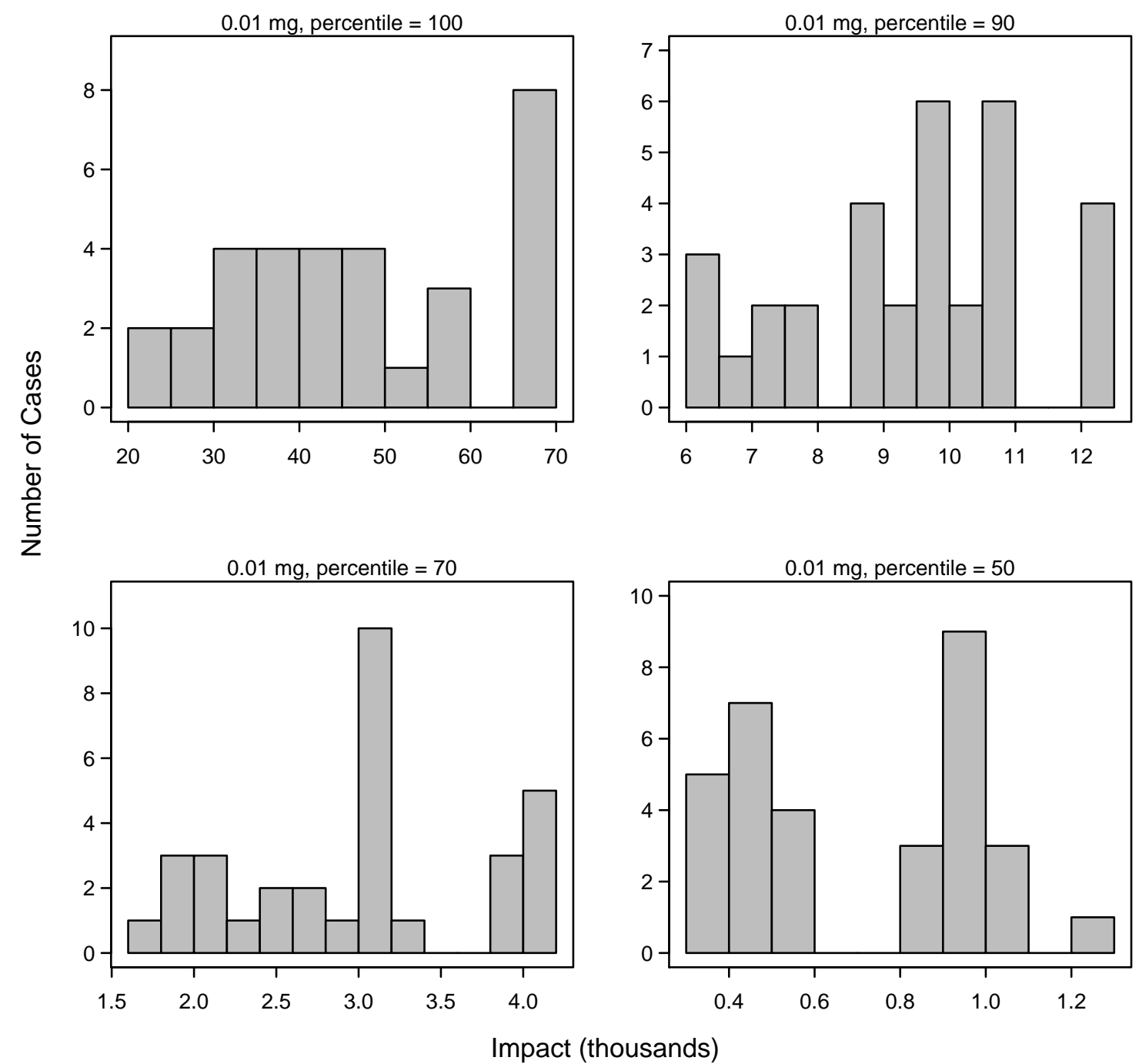

Figure 9.2. Histograms of impacts for the 32 combinations of factors for Network 2 for a dose level of $0.01 \mathrm{mg}$ and different impact levels. The number of cases on the vertical axis is the number of combinations of factors, out of a total of 32, that are in a particular bin in a histogram. 

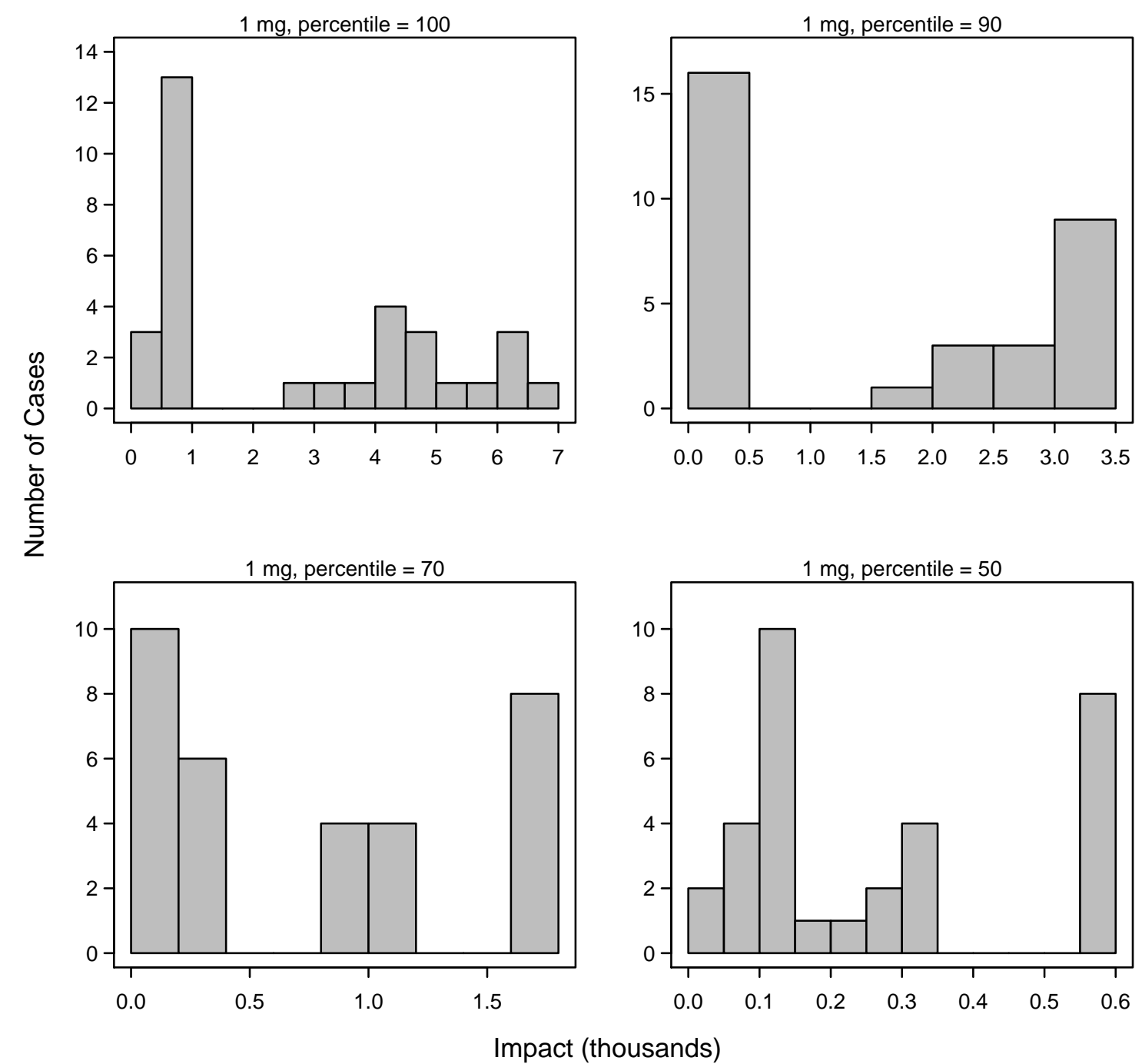

Figure 9.3. Histograms of impacts for the 32 combinations of factors for Network 2 for a dose level of $1 \mathrm{mg}$ and different impact levels. The number of cases on the vertical axis is the number of combinations of factors, out of a total of 32, that are in a particular bin in a histogram. 

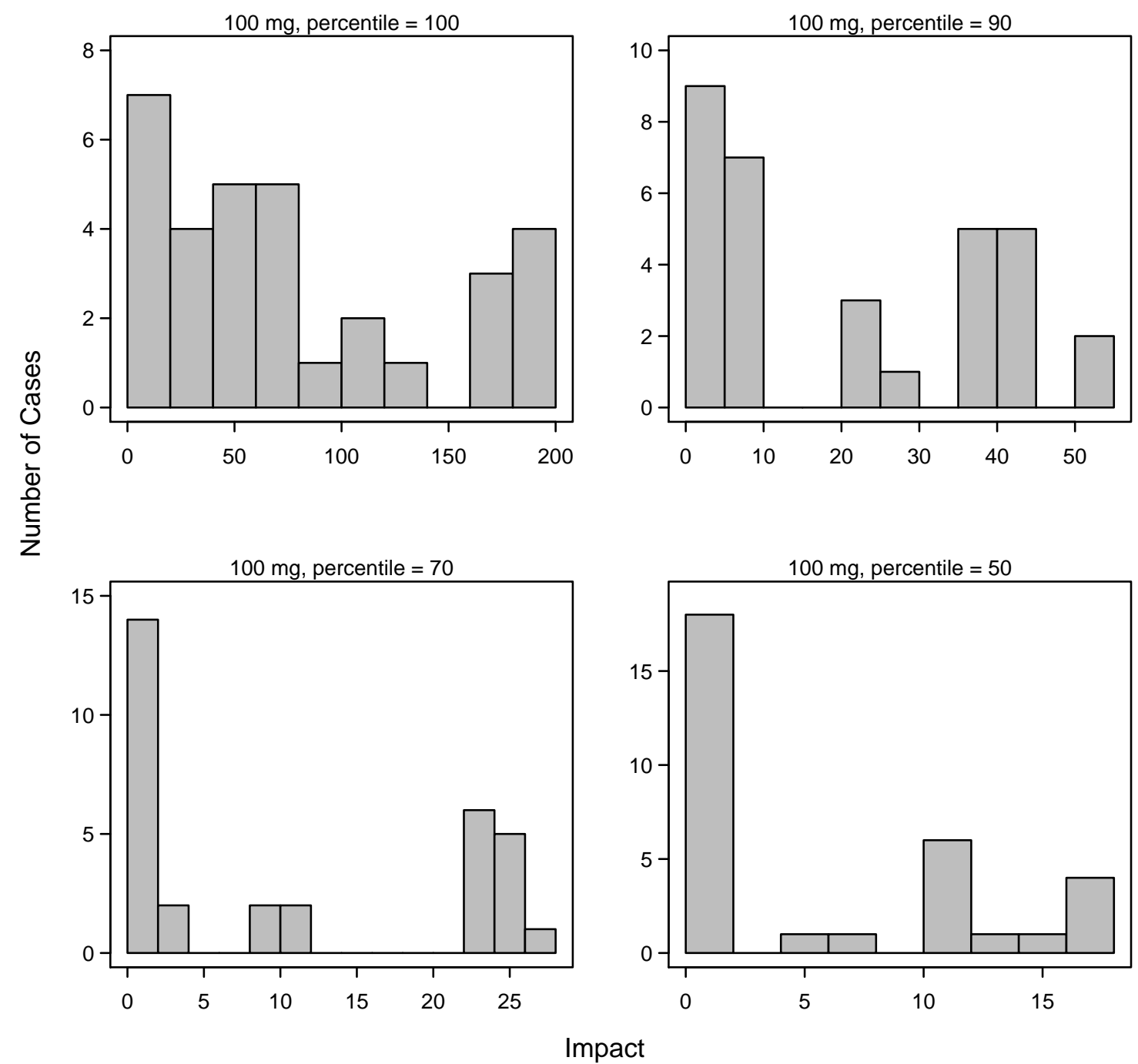

Figure 9.4. Histograms of impacts for the 32 combinations of factors for Network 2 for a dose level of $100 \mathrm{mg}$ and different impact levels. The number of cases on the vertical axis is the number of combinations of factors, out of a total of 32, that are in a particular bin in a histogram. 

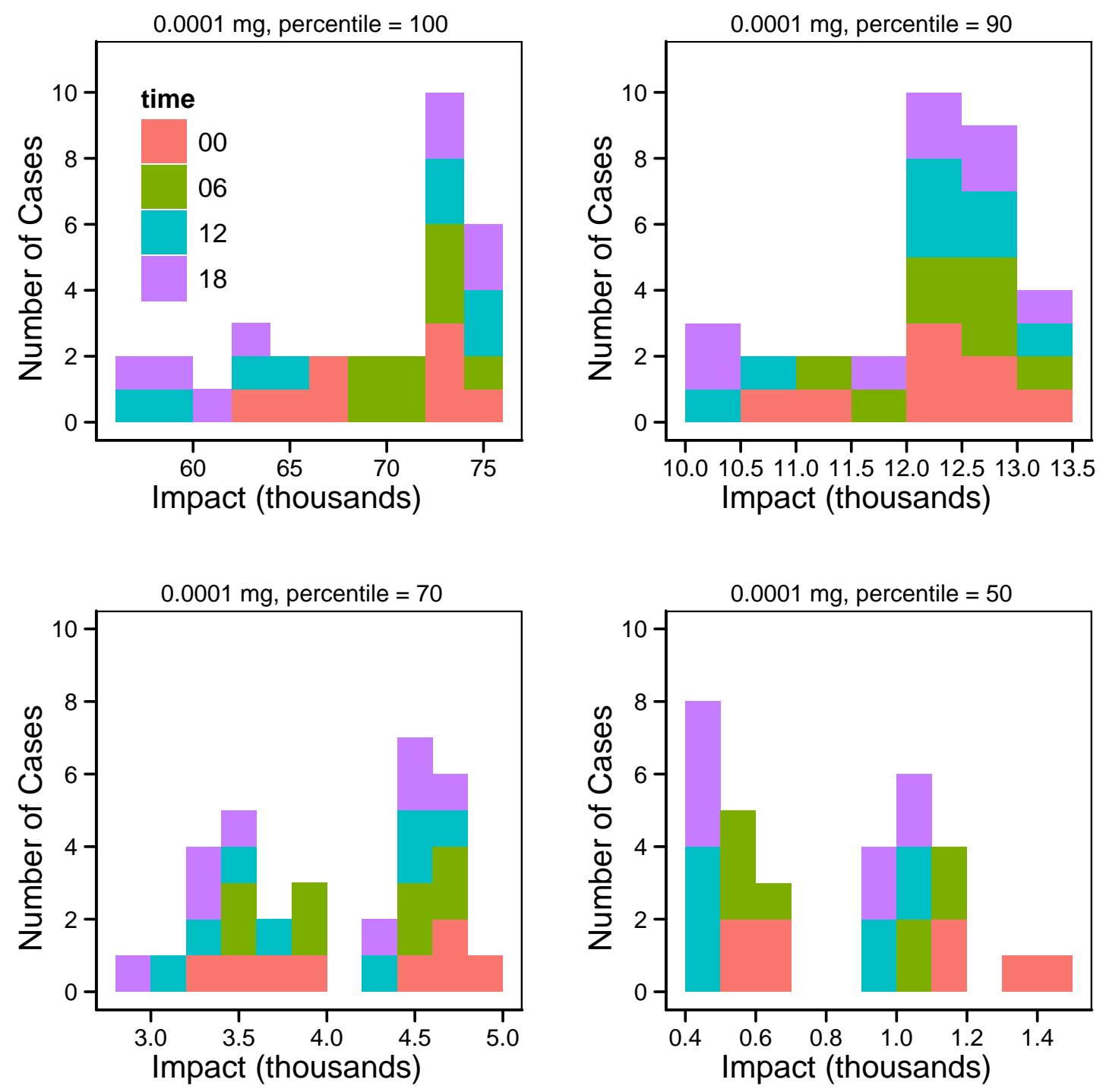

Figure 9.5. Histograms of impacts for the 32 combinations of factors for Network 2 for a dose level of $0.0001 \mathrm{mg}$ and different impact levels with cases color coded to show associated injection time. The number of cases on the vertical axis is the number of combinations of factors, out of a total of 32, that are in a particular bin in a histogram. 

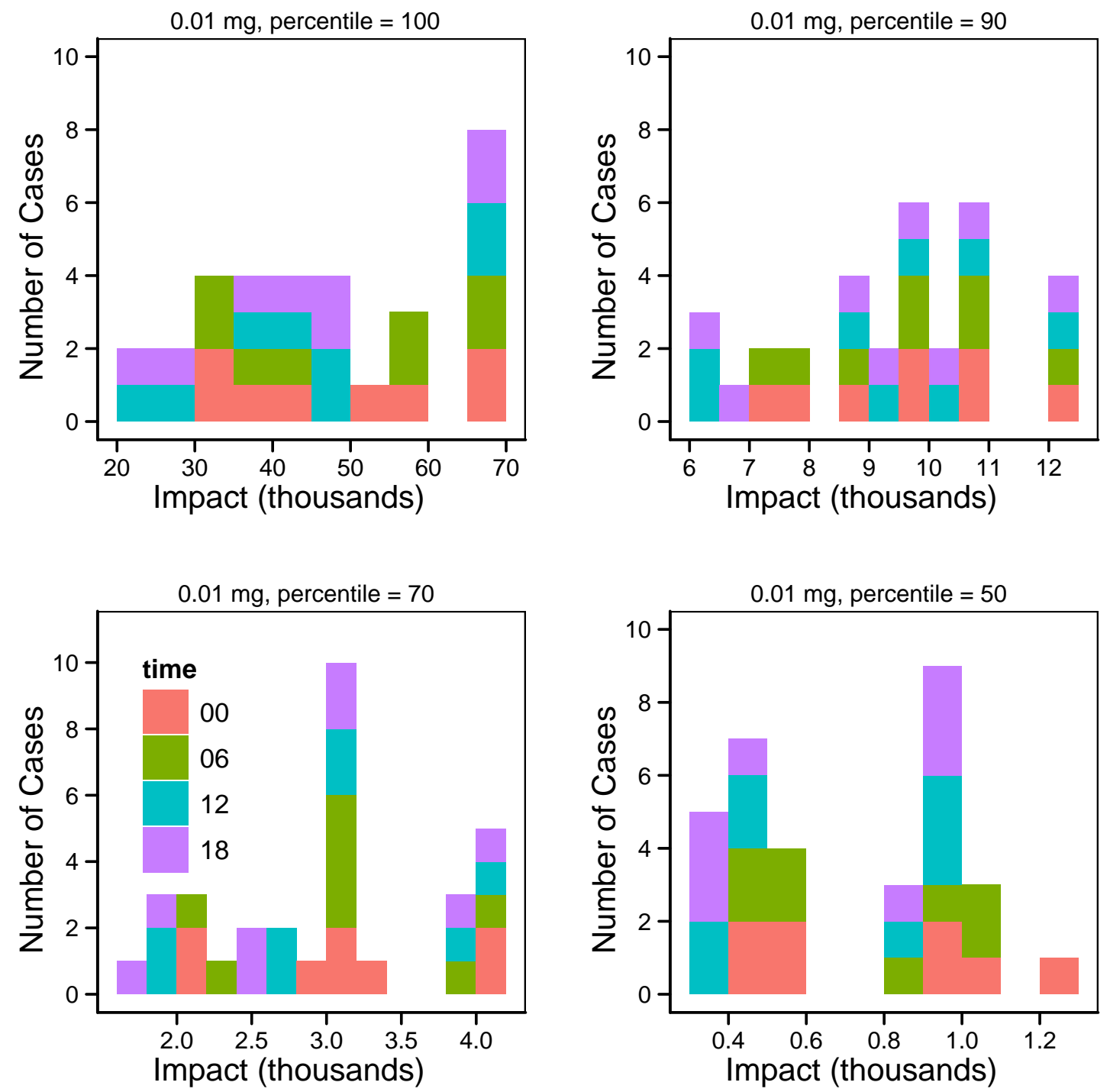

Figure 9.6. Histograms of impacts for the 32 combinations of factors for Network 2 for a dose level of $0.01 \mathrm{mg}$ and different impact levels with cases color coded to show associated injection time. The number of cases on the vertical axis is the number of combinations of factors, out of a total of 32, that are in a particular bin in a histogram. 

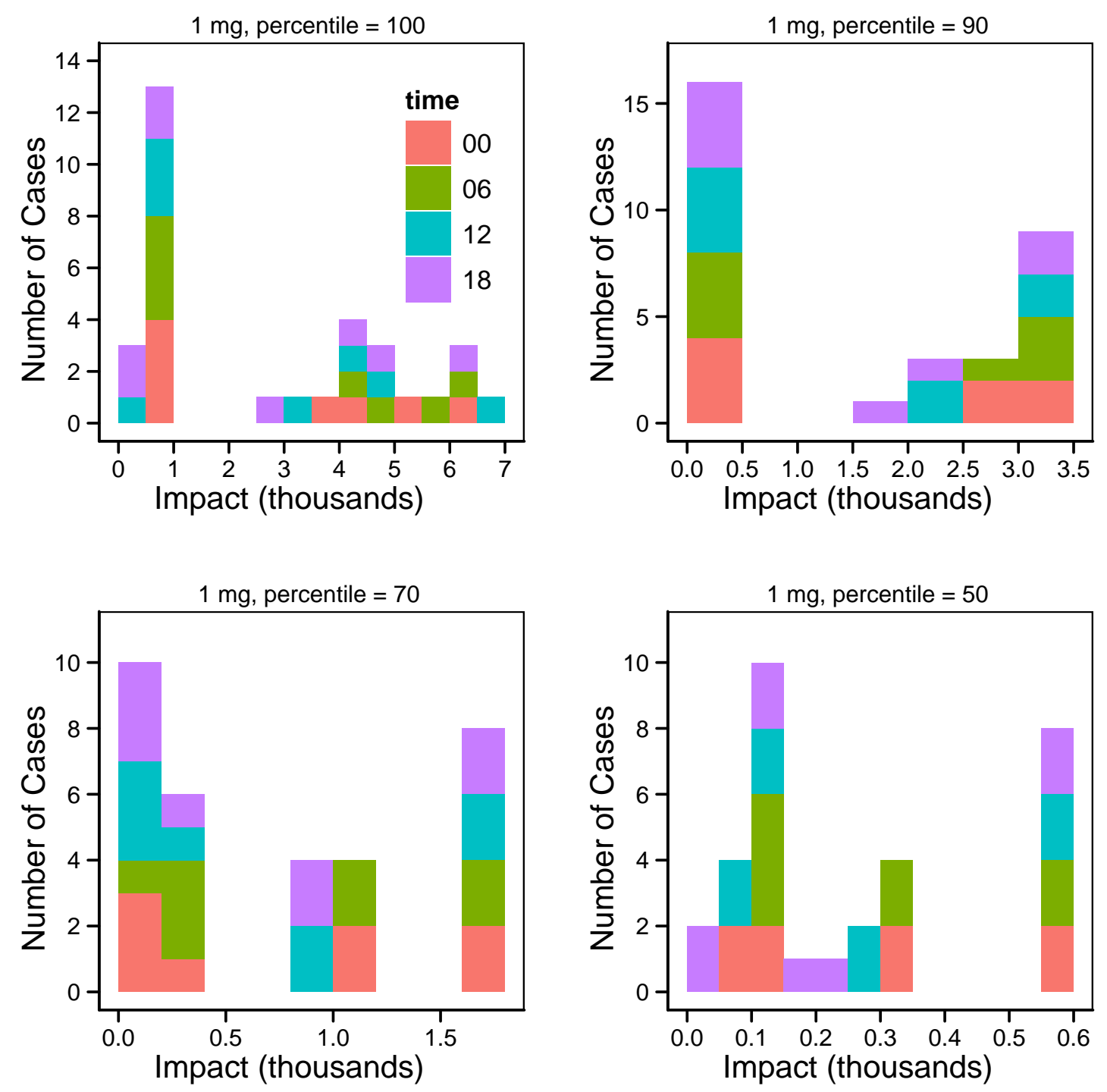

Figure 9.7. Histograms of impacts for the 32 combinations of factors for Network 2 for a dose level of $1 \mathrm{mg}$ and different impact levels with cases color coded to show associated injection time. The number of cases on the vertical axis is the number of combinations of factors, out of a total of 32, that are in a particular bin in a histogram. 

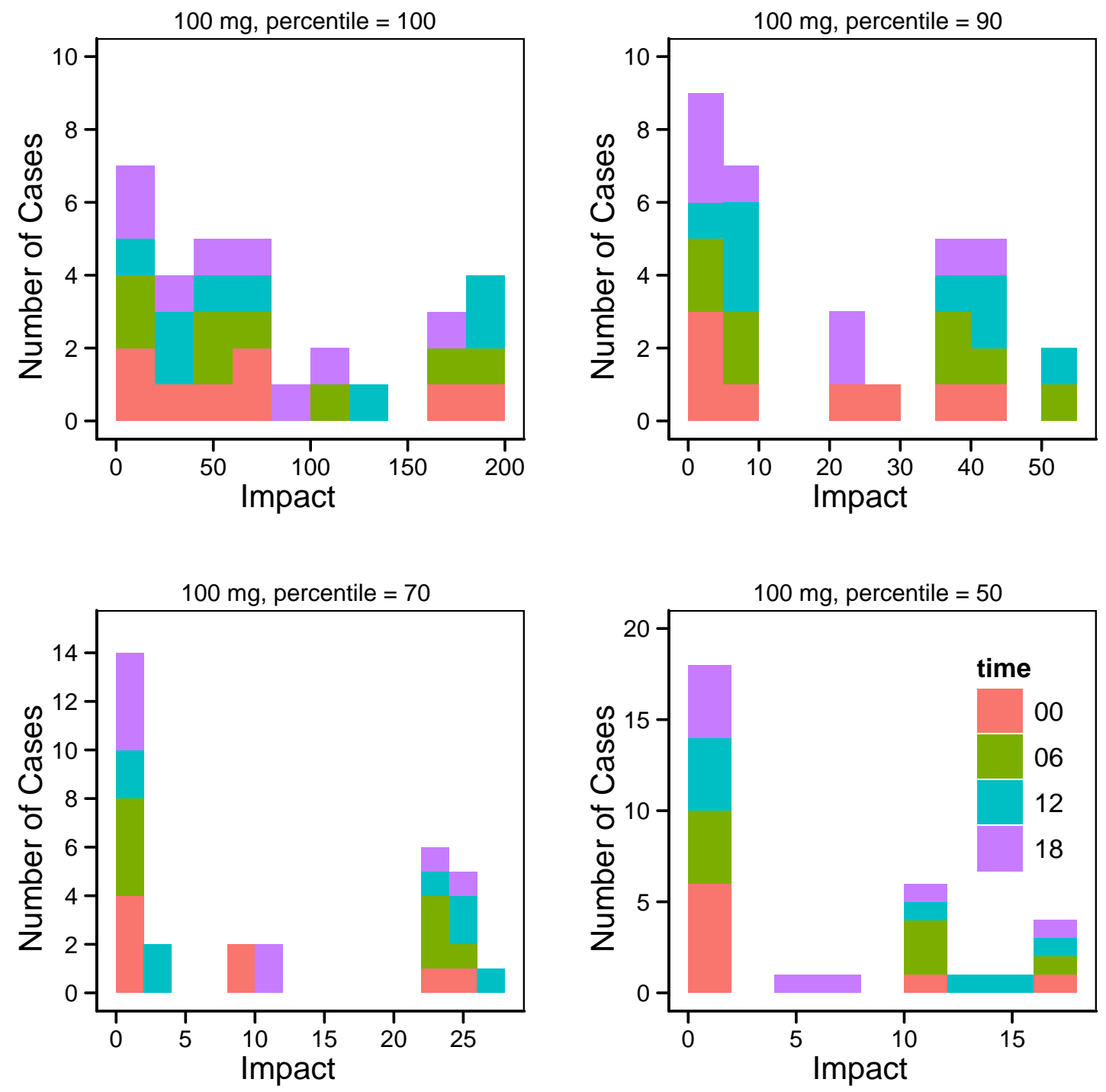

Figure 9.8. Histograms of impacts for the 32 combinations of factors for Network 2 for a dose level of $100 \mathrm{mg}$ and different impact levels with cases color coded to show associated injection time. The number of cases on the vertical axis is the number of combinations of factors, out of a total of 32, that are in a particular bin in a histogram. 

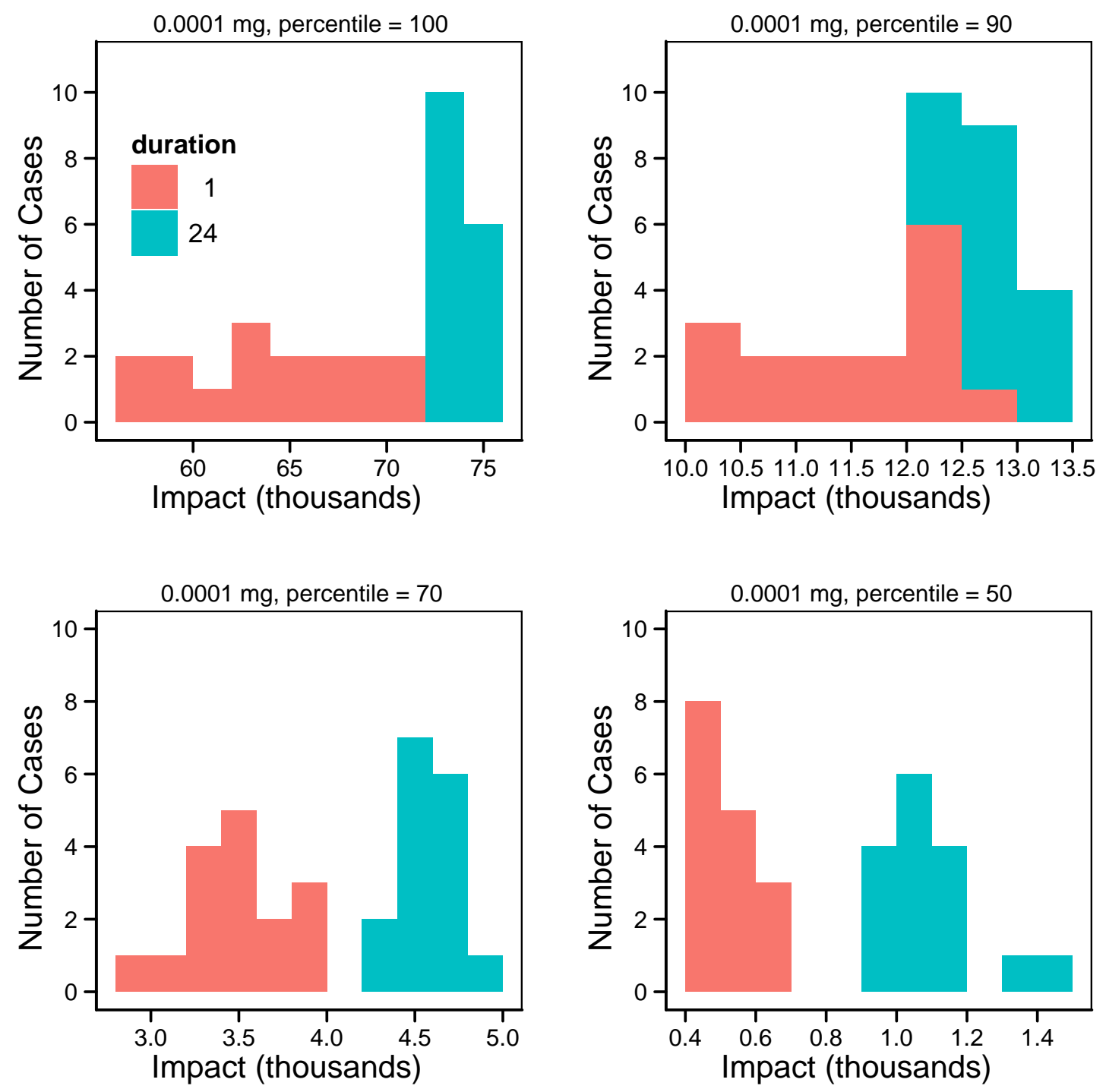

Figure 9.9. Histograms of impacts for the 32 combinations of factors for Network 2 for a dose level of $0.0001 \mathrm{mg}$ and different impact levels with cases color coded to show associated injection duration. The number of cases on the vertical axis is the number of combinations of factors, out of a total of 32, that are in a particular bin in a histogram. 

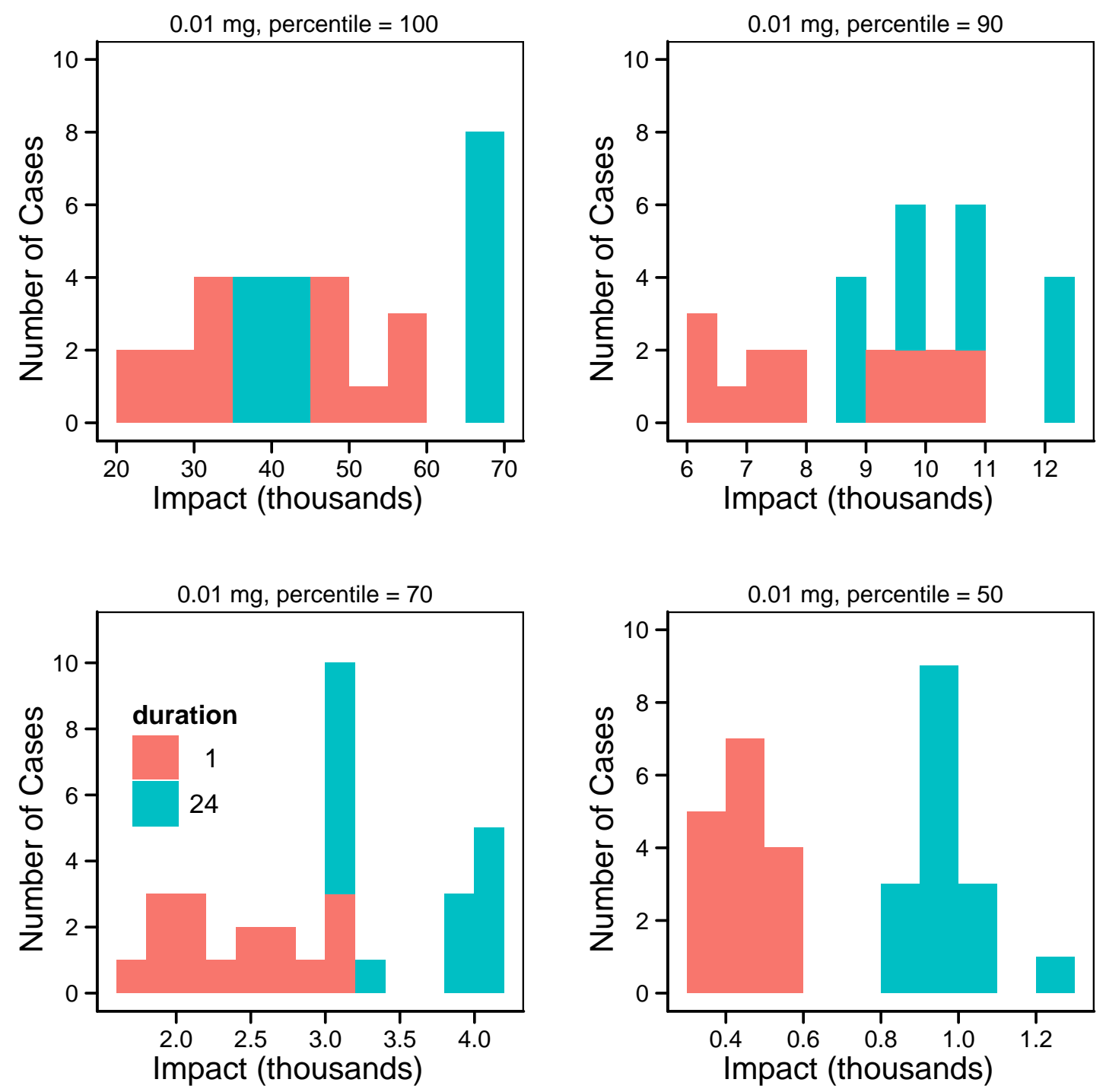

Figure 9.10. Histograms of impacts for the 32 combinations of factors for Network 2 for a dose level of $0.01 \mathrm{mg}$ and different impact levels with cases color coded to show associated injection duration. The number of cases on the vertical axis is the number of combinations of factors, out of a total of 32, that are in a particular bin in a histogram. 

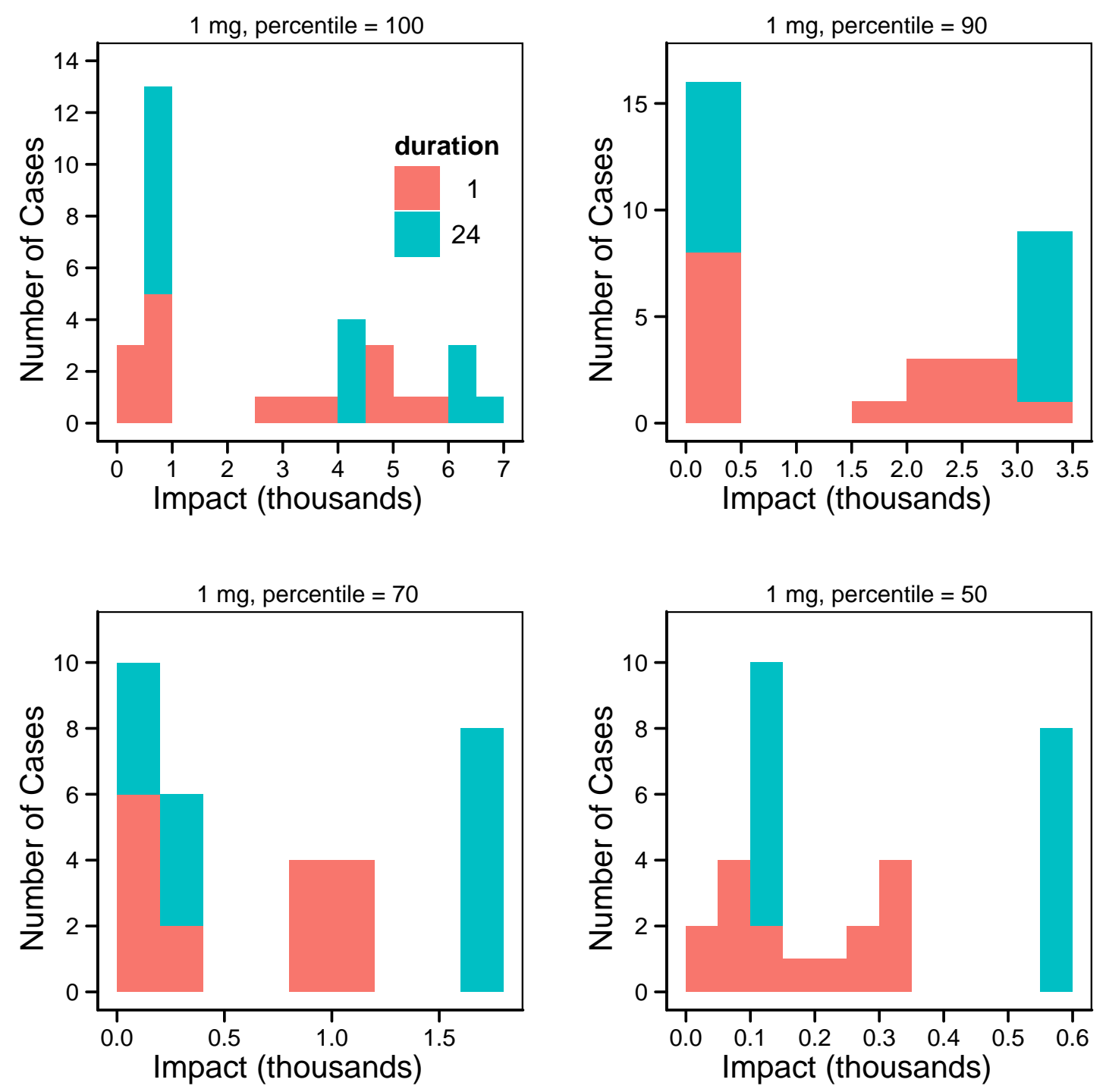

Figure 9.11. Histograms of impacts for the 32 combinations of factors for Network 2 for a dose level of $1 \mathrm{mg}$ and different impact levels with cases color coded to show associated injection duration. The number of cases on the vertical axis is the number of combinations of factors, out of a total of 32, that are in a particular bin in a histogram. 

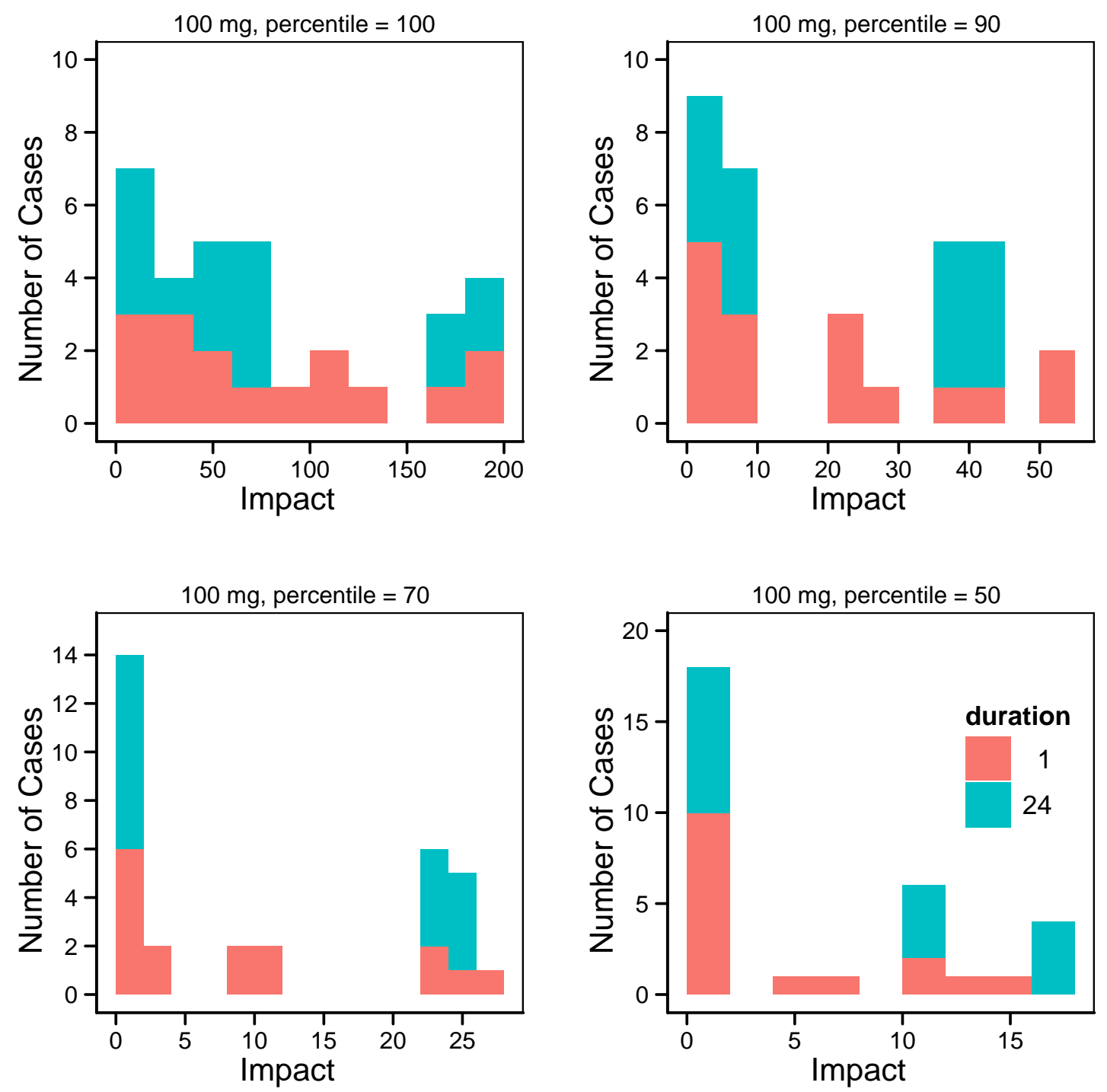

Figure 9.12. Histograms of impacts for the 32 combinations of factors for Network 2 for a dose level of $100 \mathrm{mg}$ and different impact levels with cases color coded to show associated injection duration. The number of cases on the vertical axis is the number of combinations of factors, out of a total of 32, that are in a particular bin in a histogram. 

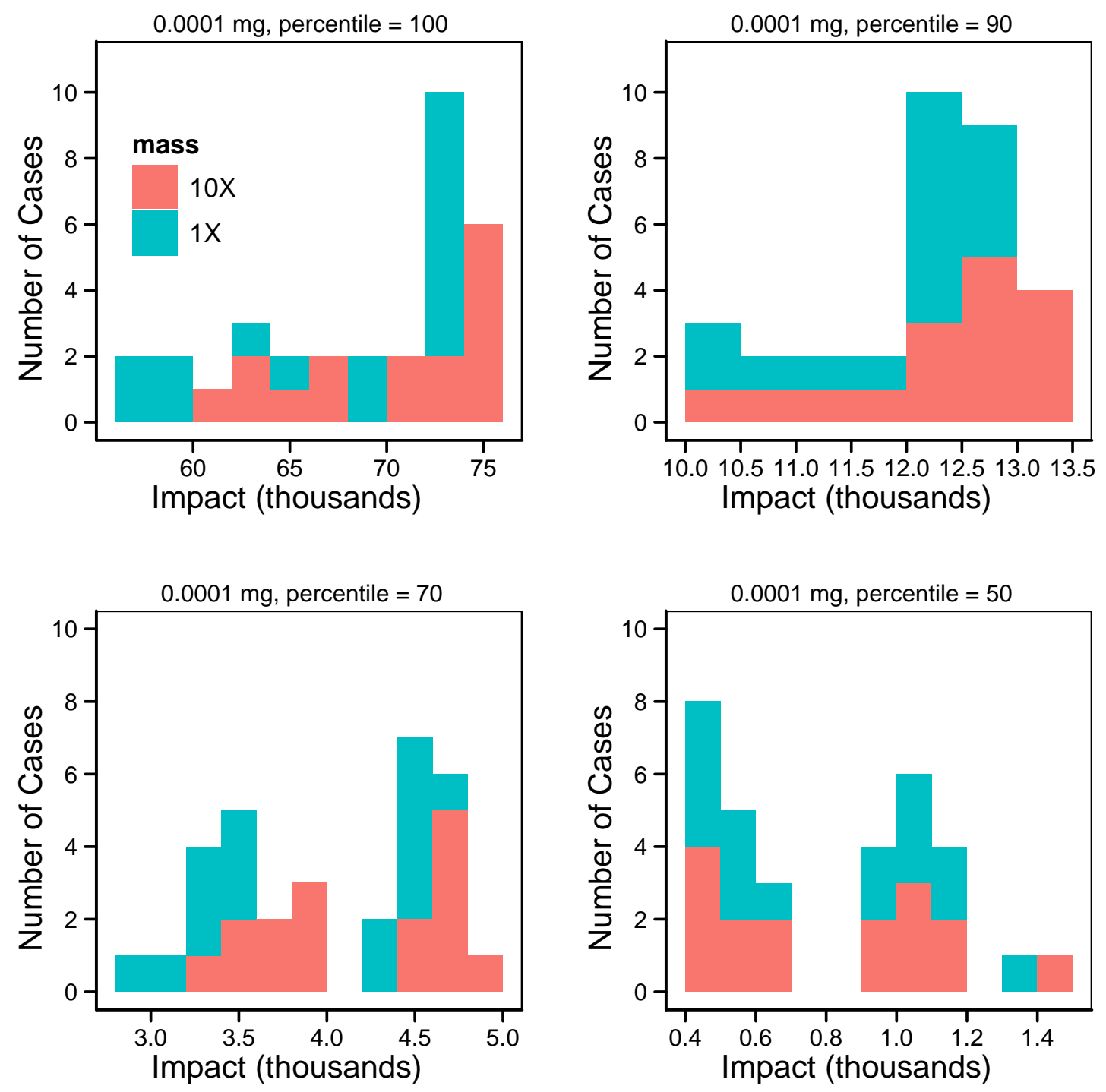

Figure 9.13. Histograms of impacts for the 32 combinations of factors for Network 2 for a dose level of $0.0001 \mathrm{mg}$ and different impact levels with cases color coded to show associated injection mass. The number of cases on the vertical axis is the number of combinations of factors, out of a total of 32, that are in a particular bin in a histogram. 

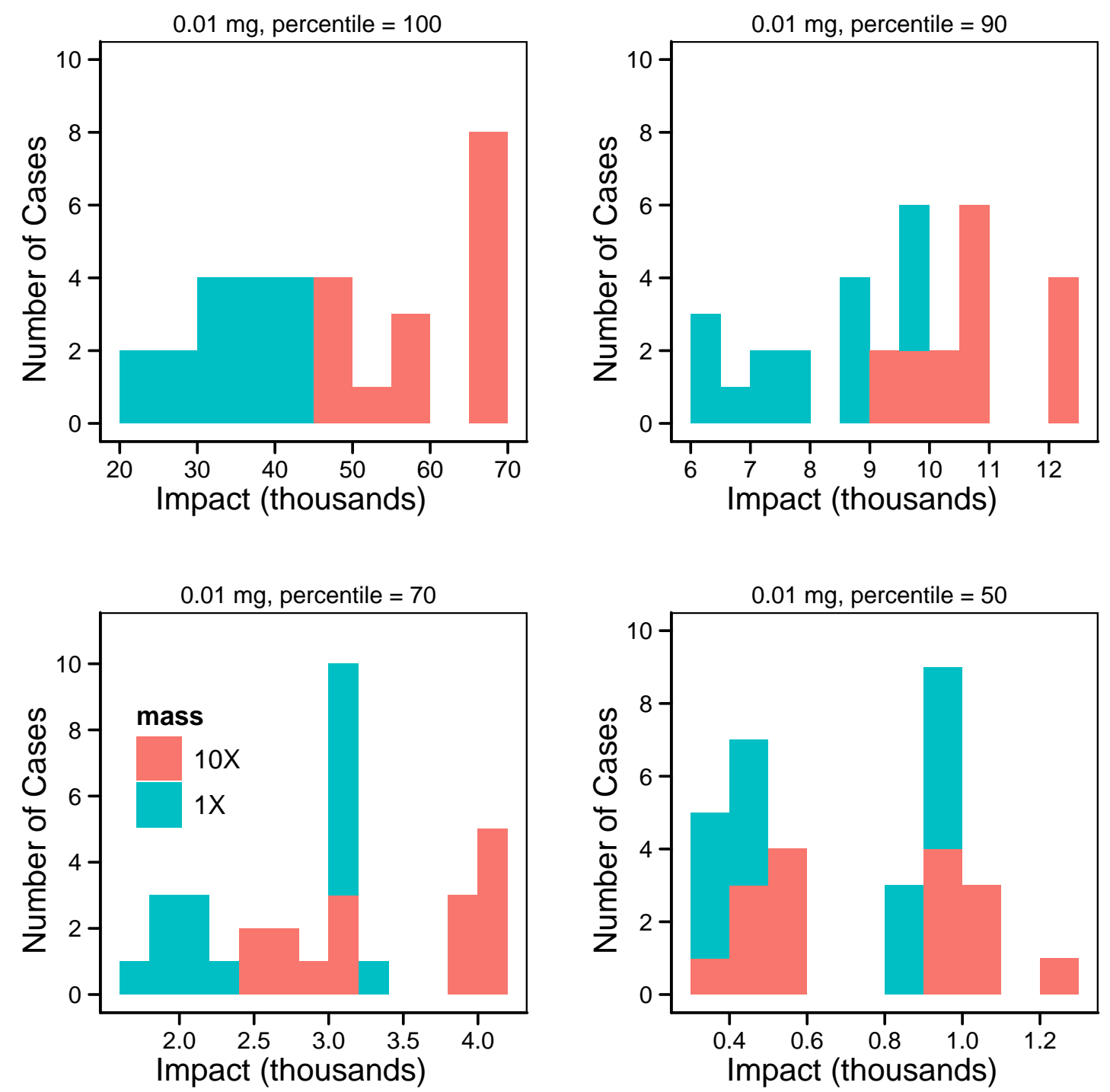

Figure 9.14. Histograms of impacts for the 32 combinations of factors for Network 2 for a dose level of $0.01 \mathrm{mg}$ and different impact levels with cases color coded to show associated injection mass. The number of cases on the vertical axis is the number of combinations of factors, out of a total of 32, that are in a particular bin in a histogram. 

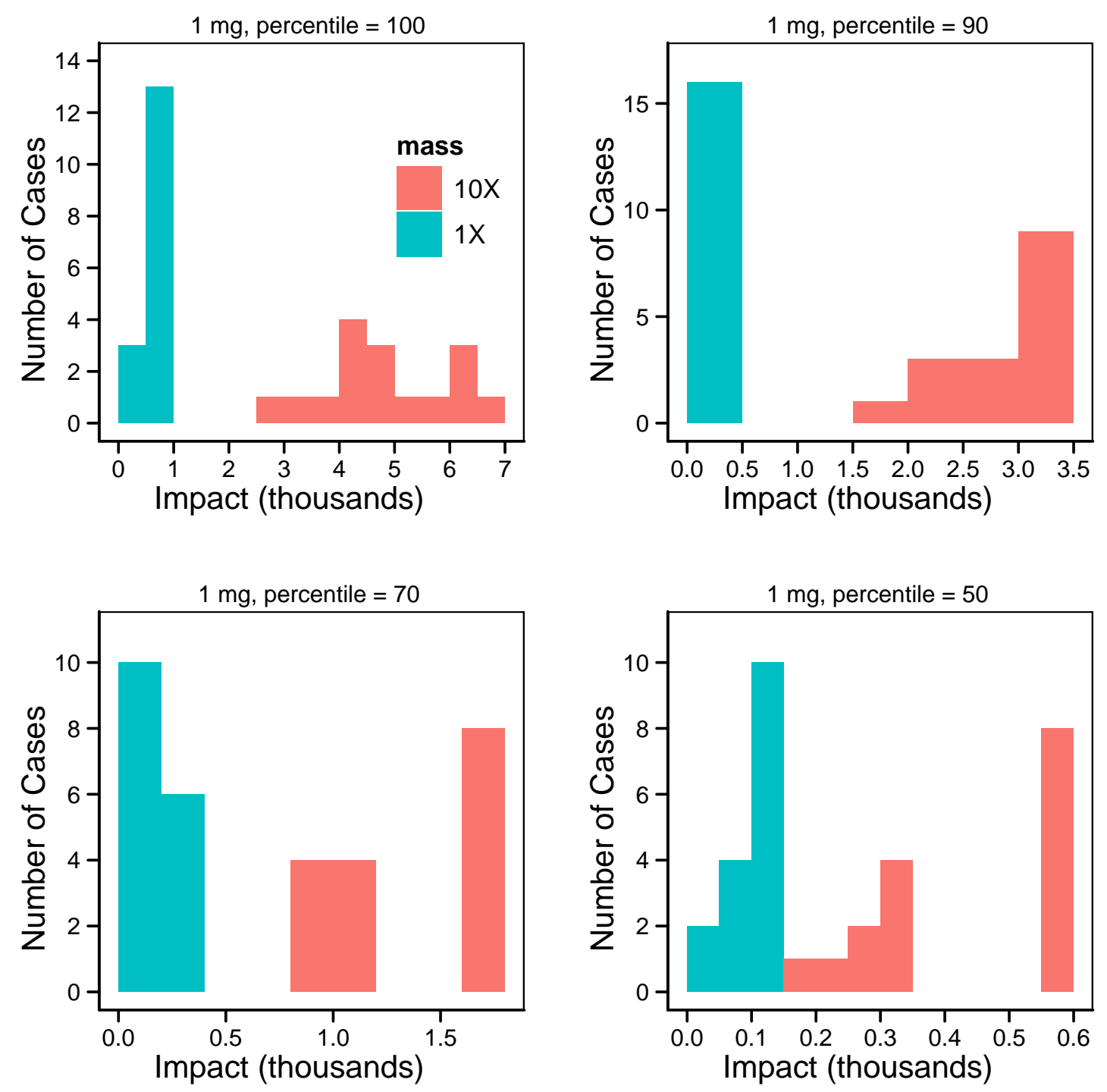

Figure 9.15. Histograms of impacts for the 32 combinations of factors for Network 2 for a dose level of $1 \mathrm{mg}$ and different impact levels with cases color coded to show associated injection mass. The number of cases on the vertical axis is the number of combinations of factors, out of a total of 32, that are in a particular bin in a histogram. 

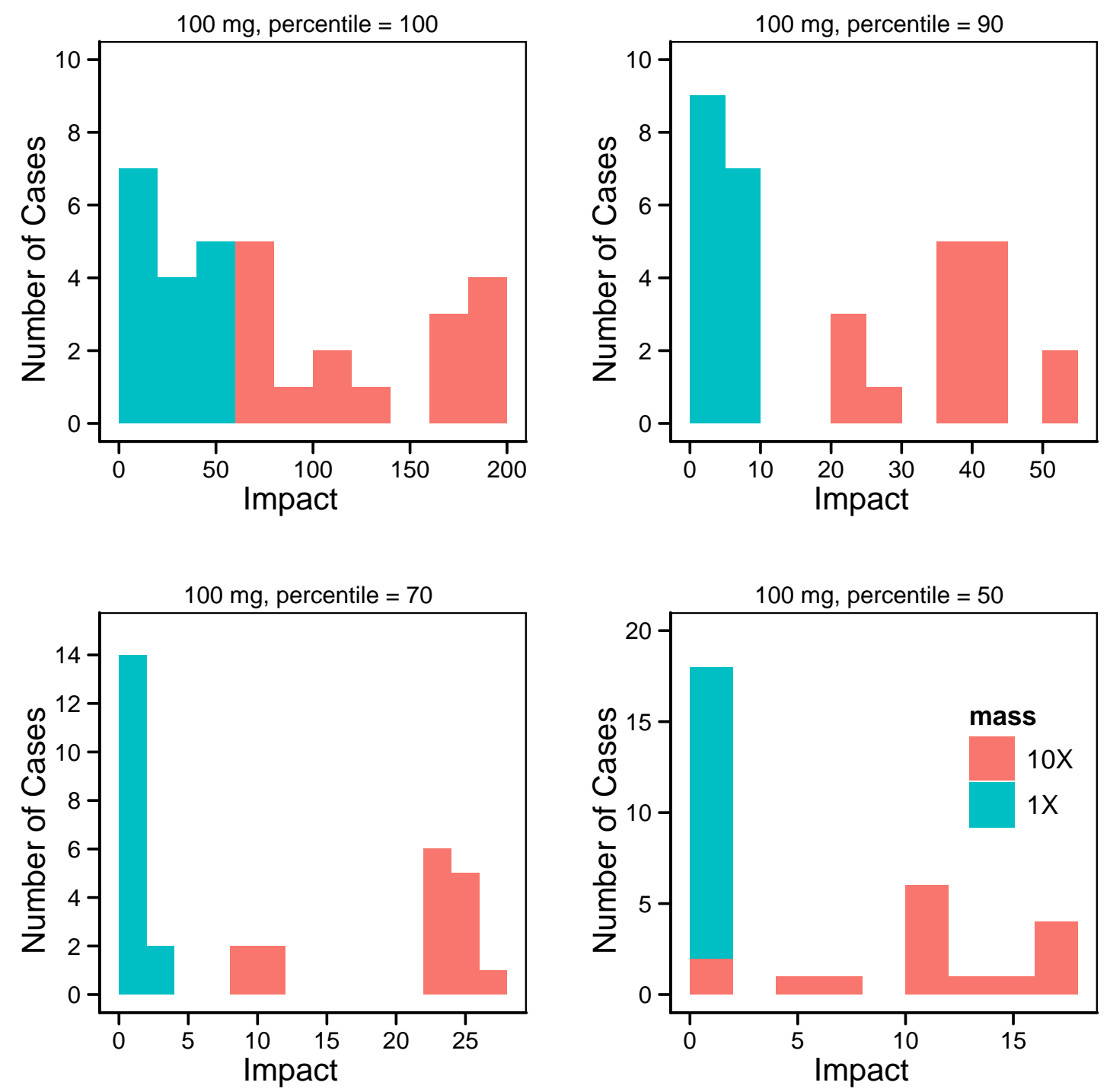

Figure 9.16. Histograms of impacts for the 32 combinations of factors for Network 2 for a dose level of $100 \mathrm{mg}$ and different impact levels with cases color coded to show associated injection mass. The number of cases on the vertical axis is the number of combinations of factors, out of a total of 32, that are in a particular bin in a histogram. 

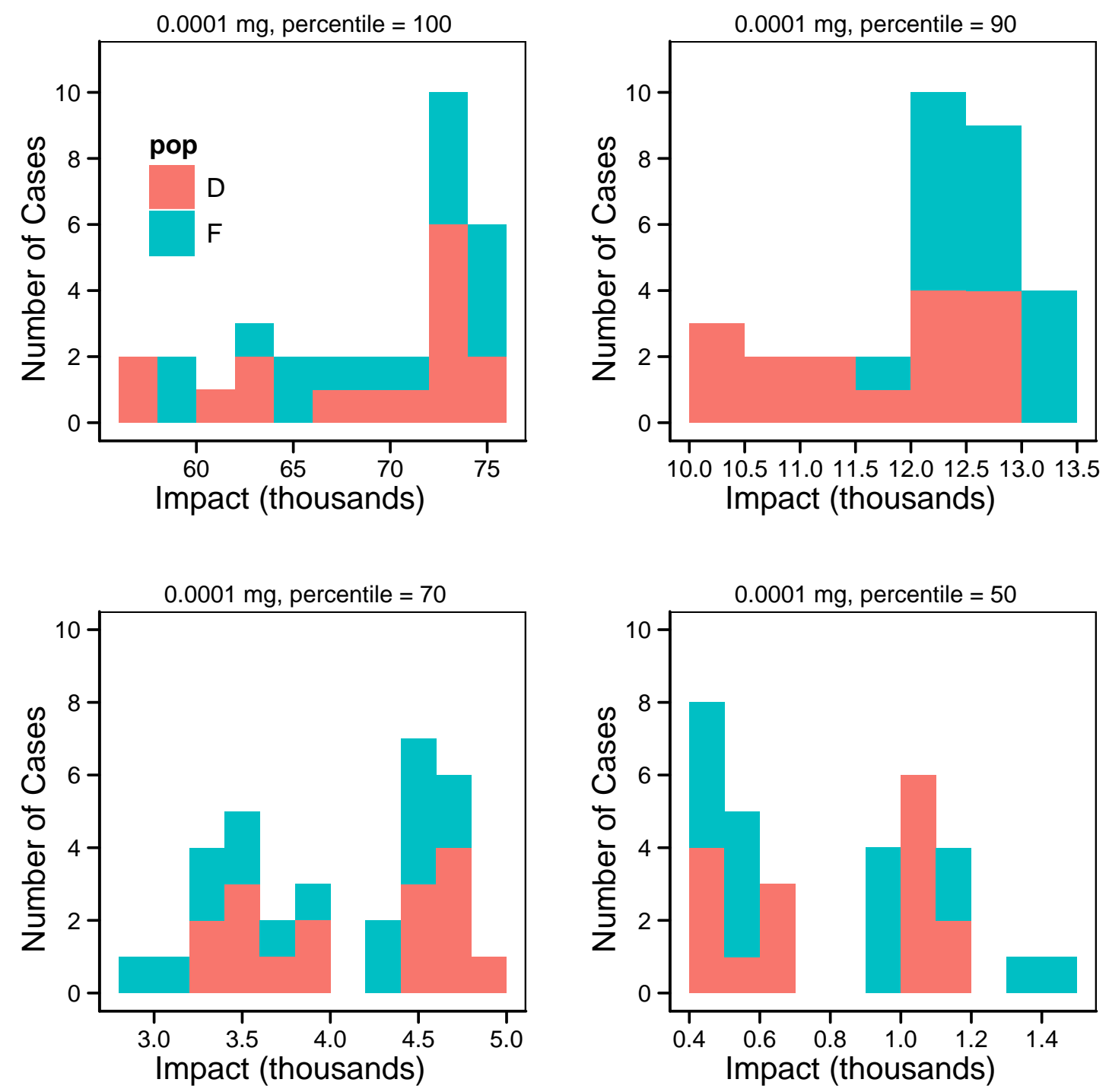

Figure 9.17. Histograms of impacts for the 32 combinations of factors for Network 2 for a dose level of $0.0001 \mathrm{mg}$ and different impact levels with cases color coded to show which population model was used. $D$ is the demand-based population model and $F$ is the population model with an average, fixed population at each node. The number of cases on the vertical axis is the number of combinations of factors, out of a total of 32, that are in a particular bin in a histogram. 

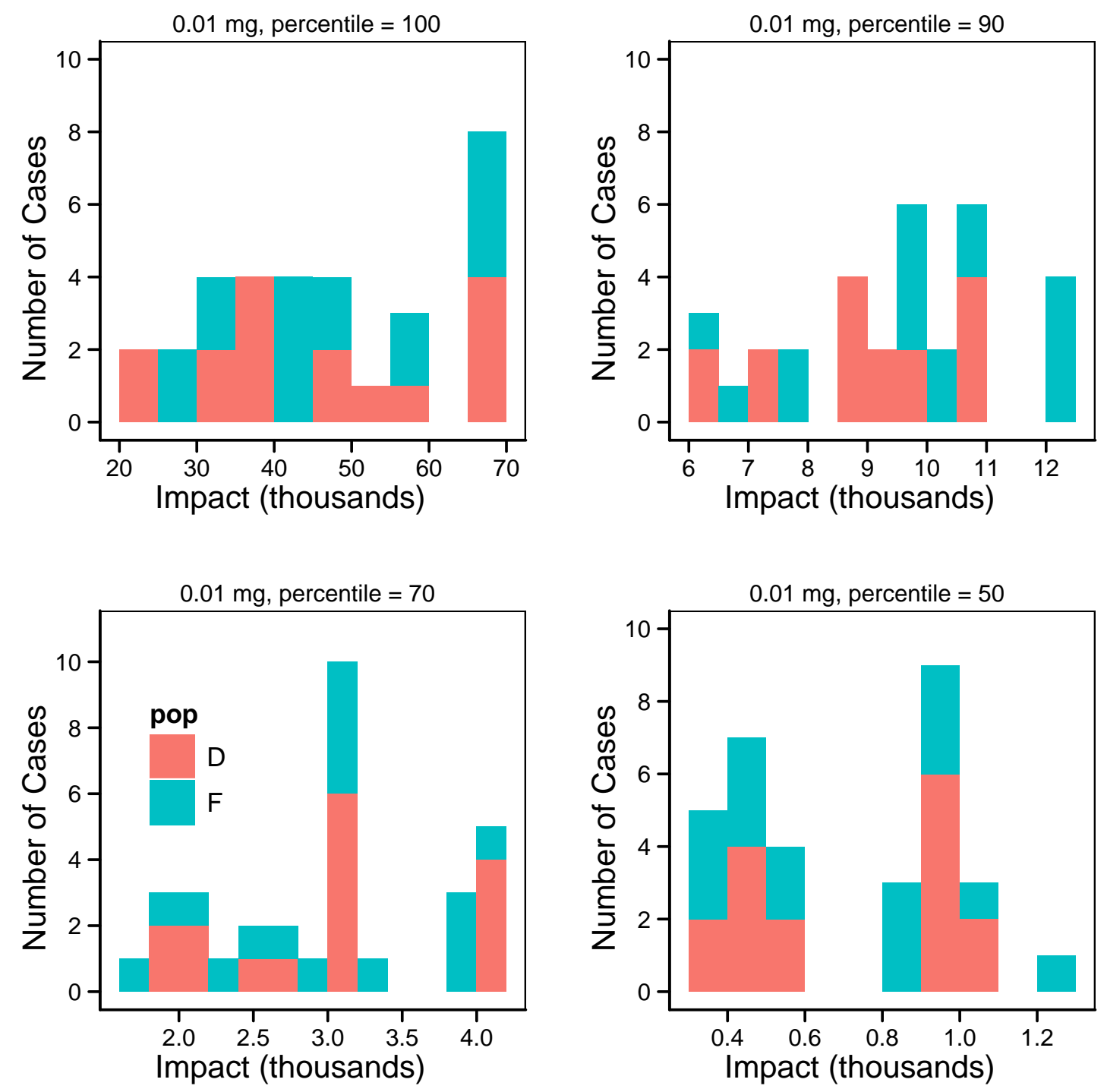

Figure 9.18. Histograms of impacts for the 32 combinations of factors for Network 2 for a dose level of $0.01 \mathrm{mg}$ and different impact levels with cases color coded to show which population model was used. $D$ is the demand-based population model and $F$ is the population model with an average, fixed population at each node. The number of cases on the vertical axis is the number of combinations of factors, out of a total of 32, that are in a particular bin in a histogram. 

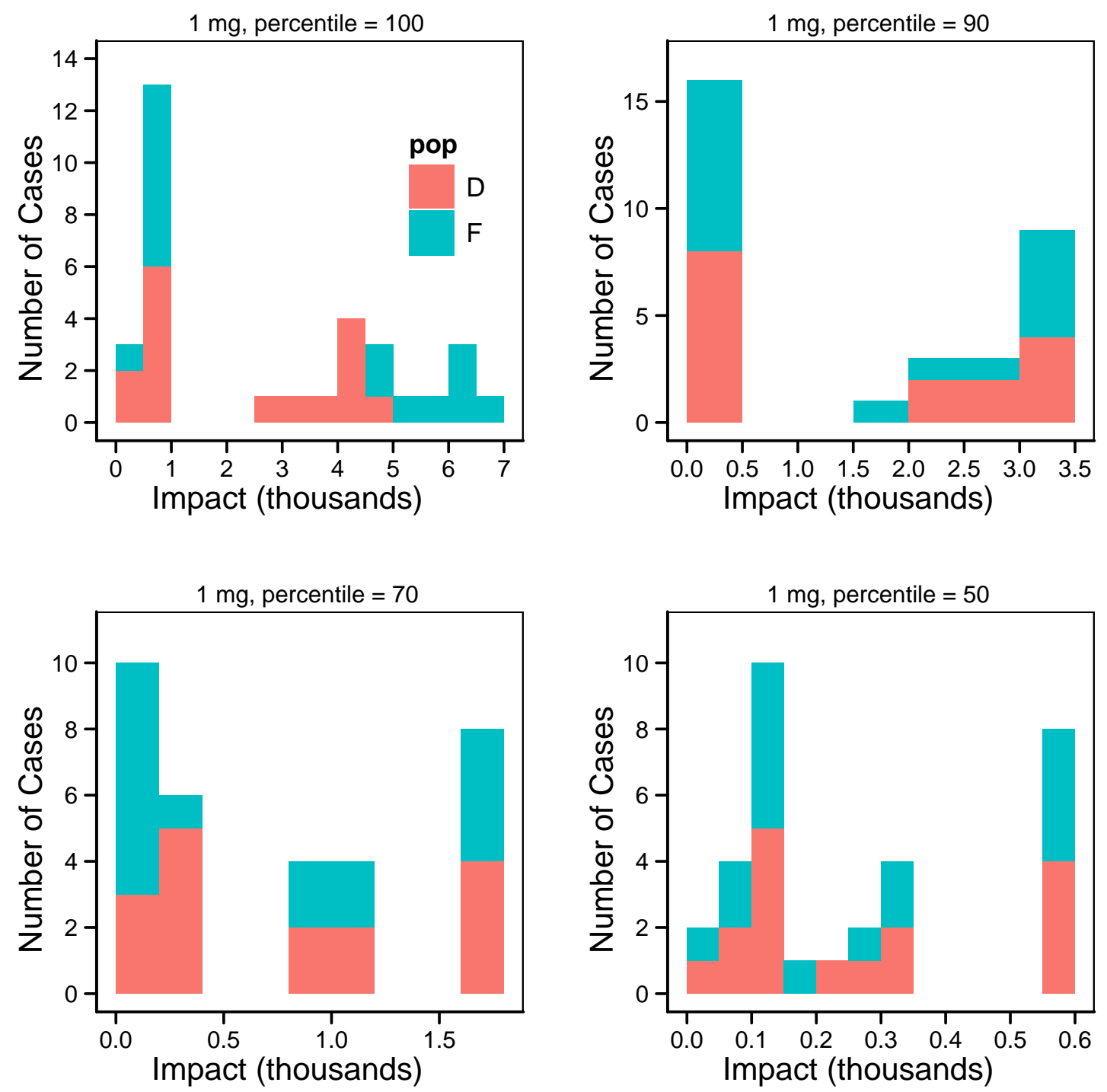

Figure 9.19. Histograms of impacts for the 32 combinations of factors for Network 2 for a dose level of $1 \mathrm{mg}$ and different impact levels with cases color coded to show which population model was used. $D$ is the demand-based population model and $F$ is the population model with an average, fixed population at each node.The number of cases on the vertical axis is the number of combinations of factors, out of a total of 32, that are in a particular bin in a histogram. 

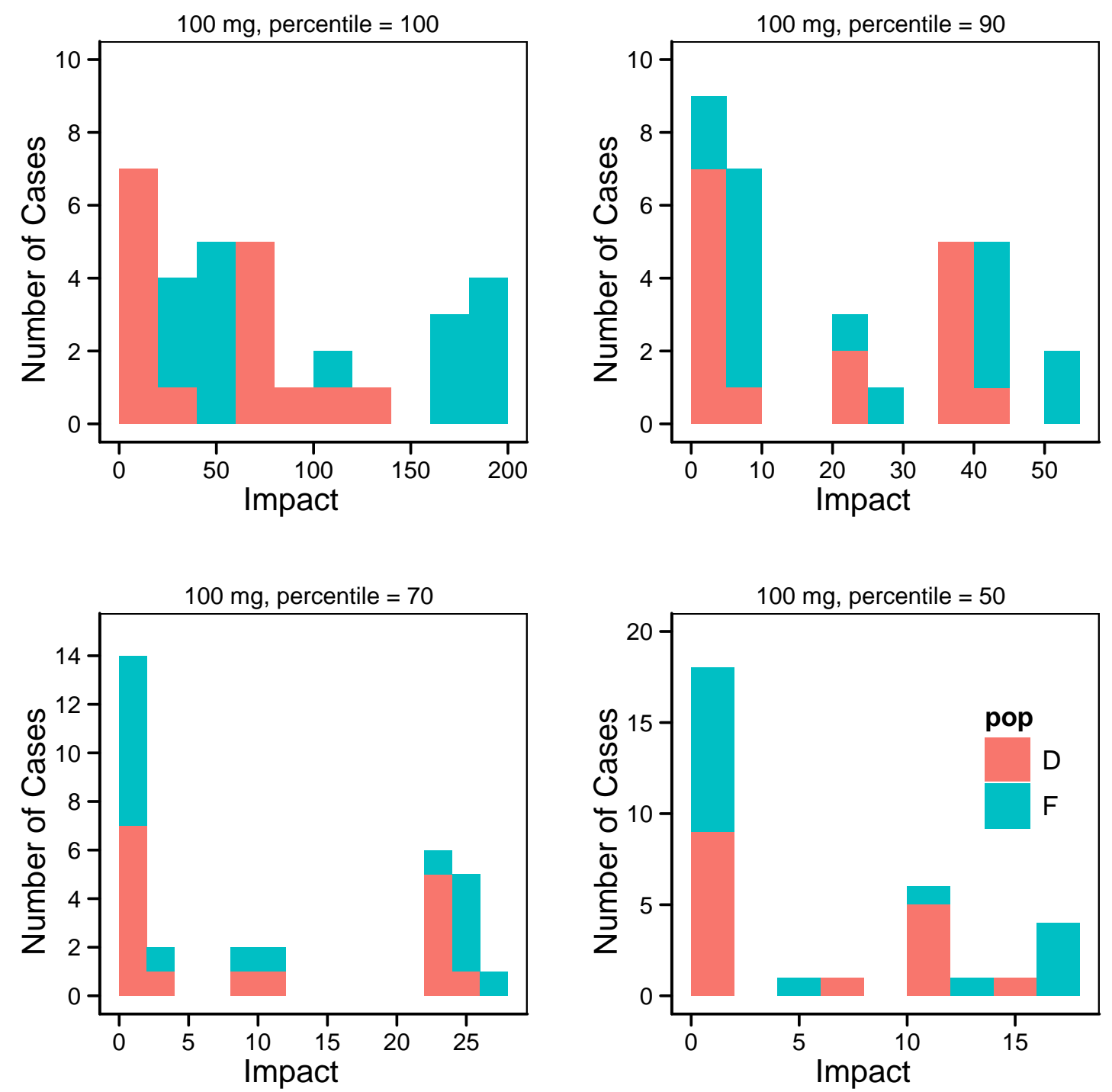

Figure 9.20. Histograms of impacts for the 32 combinations of factors for Network 2 for a dose level of $100 \mathrm{mg}$ and different impact levels with cases color coded to show which population model was used. $D$ is the demand-based population model and $F$ is the population model with an average, fixed population at each node. The number of cases on the vertical axis is the number of combinations of factors, out of a total of 32, that are in a particular bin in a histogram. 

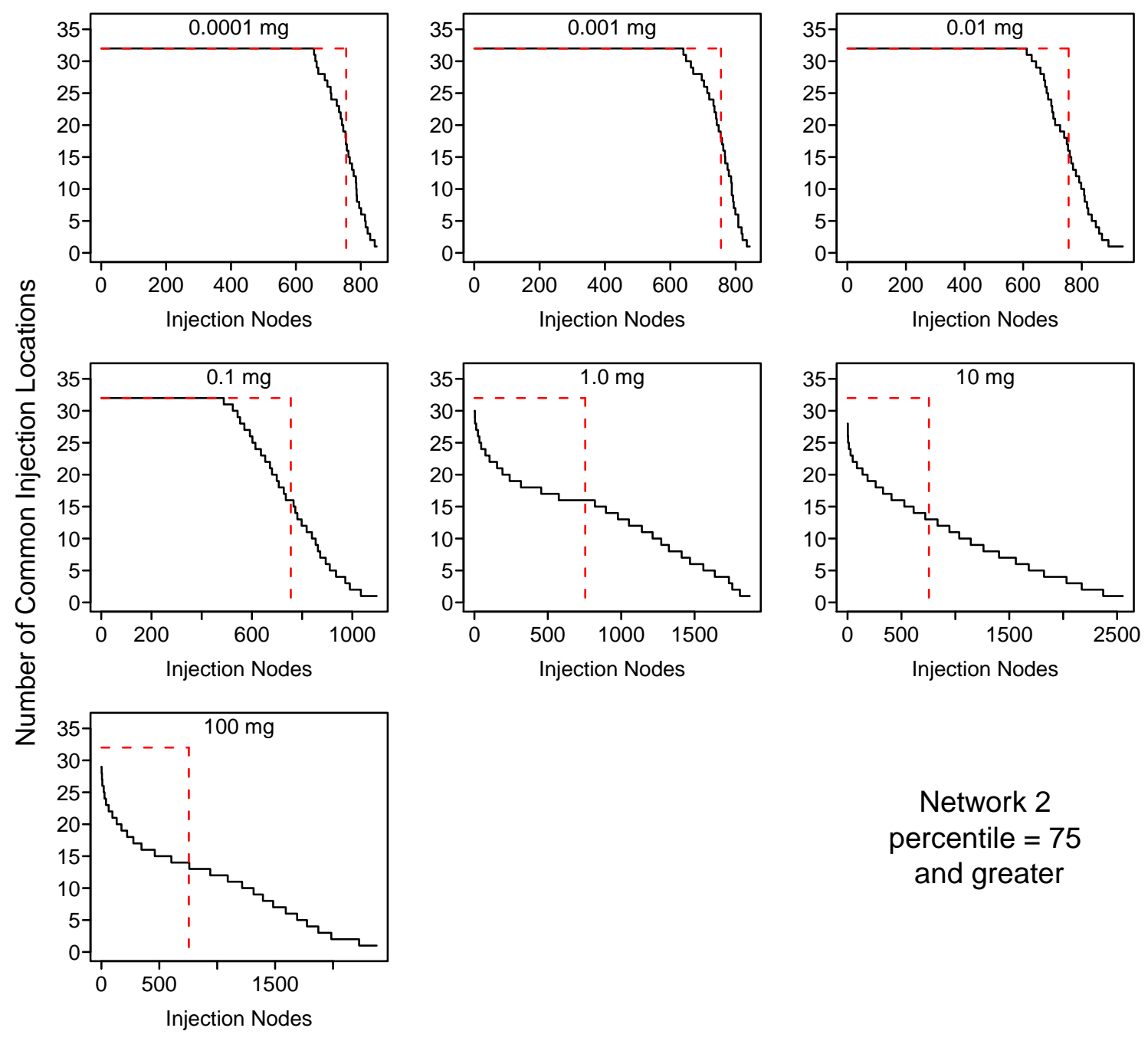

Network 2

percentile $=75$

and greater

Figure 9.21. Overlap of injection nodes for the 32 combinations of factors for Network 2 for different dose levels for $75^{\text {th }}$ percentile and higher impacts. The dashed red line illustrates results for perfect overlap of the injection nodes for all cases. The parameter in the plots is the dose level. 

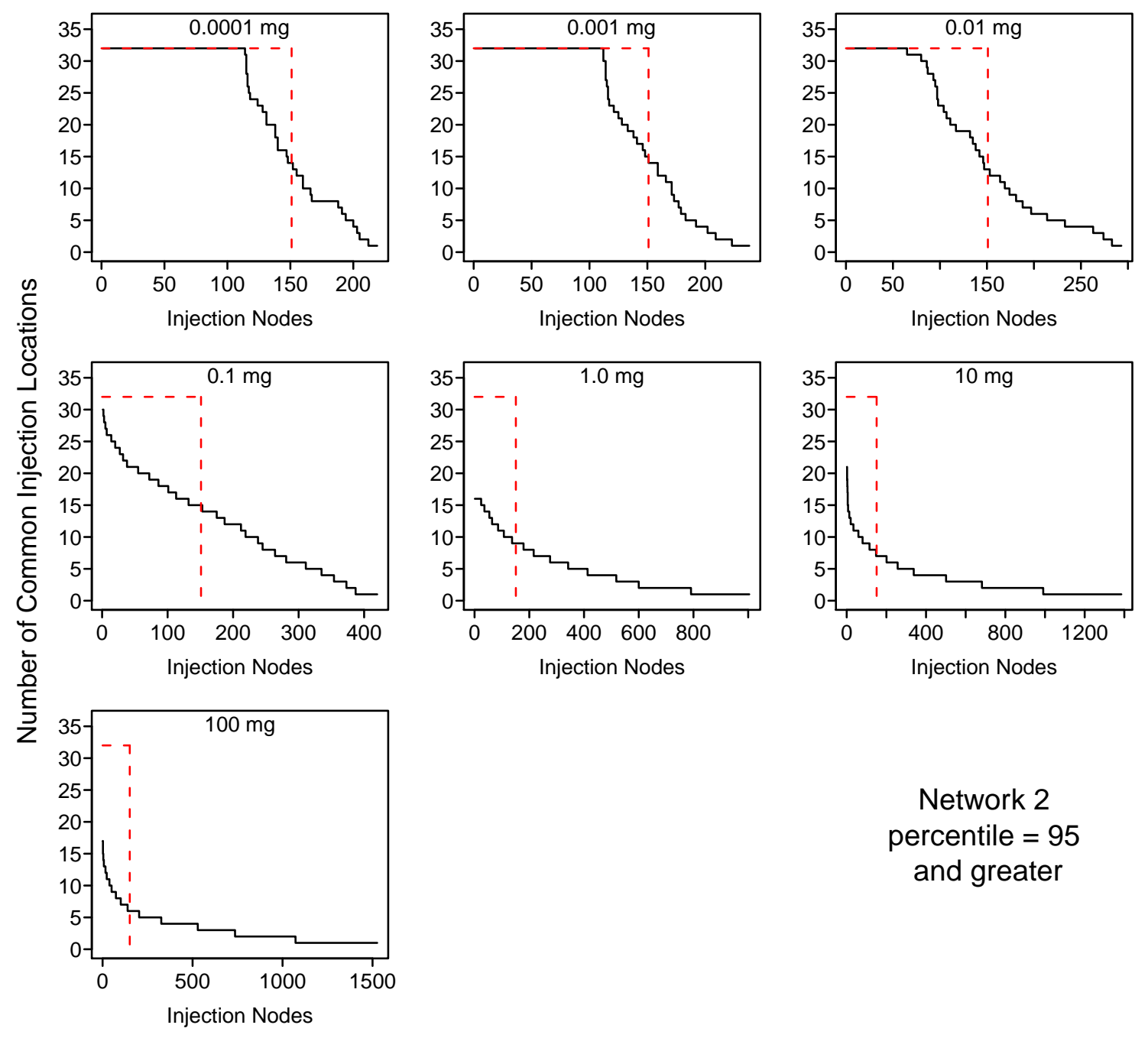

Network 2

percentile $=95$

and greater

Figure 9.22. Overlap of injection nodes for the 32 combinations of factors for Network 2 for different dose levels for $9^{\text {th }}$ percentile and higher impacts. The dashed red line illustrates results for perfect overlap of the injection nodes for all cases. The parameter in the plots is the dose level. 

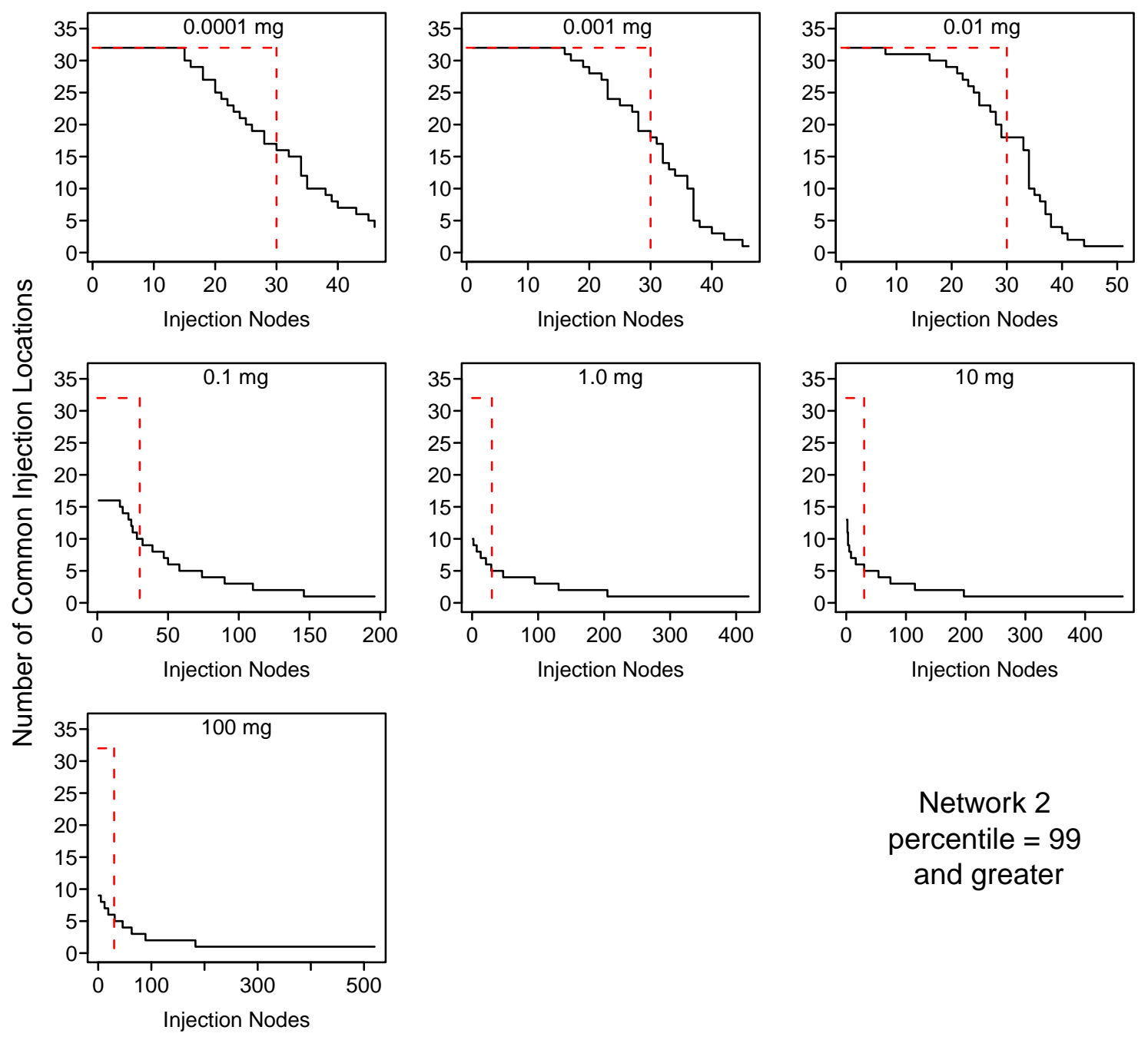

Network 2

percentile $=99$

and greater

Figure 9.23. Overlap of injection nodes for the 32 combinations of factors for Network 2 for different dose levels for $99^{\text {th }}$ percentile and higher impacts. The dashed red line illustrates results for perfect overlap of the injection nodes for all cases. The parameter in the plots is the dose level. 


\section{Section 10}

\section{Sensitivities Examined}

We examined two types of sensitivities to the various factors: sensitivity that results in variations in the magnitudes of the $n^{\text {th }}$ percentile impact and sensitivity that results in changes in the injection nodes that are associated with the $n^{\text {th }}$ percentile and higher impacts. Sensitivity involving injection nodes was examined quantitatively using the overlap of high percentile injection nodes and qualitatively using maps of injection nodes for two of the networks.

Figures 10.1 through 10.5 summarize the sensitivity of impacts to the various individual factors for all of the networks. Results are shown for the $95^{\text {th }}$ percentile and two dose levels $(0.0001$ and $1.0 \mathrm{mg}$ ). The ratios of impacts are generally about 5 or less except for injection mass, for which the ratios can exceed 10 for a dose level of $1.0 \mathrm{mg}$.

Figures 10.6 through 10.12 summarize the overlaps of injection nodes for the various individual factors. Again, results are shown for the $95^{\text {th }}$ percentile and two dose levels $(0.0001$ and $1.0 \mathrm{mg})$.

As can be seen from the figures, the sensitivity of impacts and degree of overlap vary from network to network and depend on the dose level. The figures also provide plots showing how the results are related to the areas, populations, and average population densities of the networks. There is no obvious relationship between any of these quantities and the sensitivity to the various factors or the degree of overlap of injection nodes exhibited by the networks. The inter-network variability does not appear to be related to these quantities.

The results presented in Sections 4 through 9 show that not only do the magnitudes of impacts vary with dose level and impact level (e.g., $95^{\text {th }}$ versus $99^{\text {th }}$ percentile), but the sensitivity of impacts to the various factors considered also varies with those same quantities. Impacts are sensitive to all the factors and the sensitivity depends on the network. For all factors, sensitivity to the factor tends to increase with dose level. With the exception of the population model, sensitivity to the various factors tends to be high at low impact levels. For the population model, sensitivity is lowest at the lower impact levels.

The overlap of high percentile injection nodes for different values of the same factor (e.g., 1-h and 24-h injection durations or $1 \mathrm{X}$ and $10 \mathrm{X}$ mass injections) can vary substantially with dose level and impact level, as shown in Sections 4 through 9. Overlap tends to decrease with both increasing dose level and increasing impact level. The sensitivity of overlap to all the factors varies with the network. 


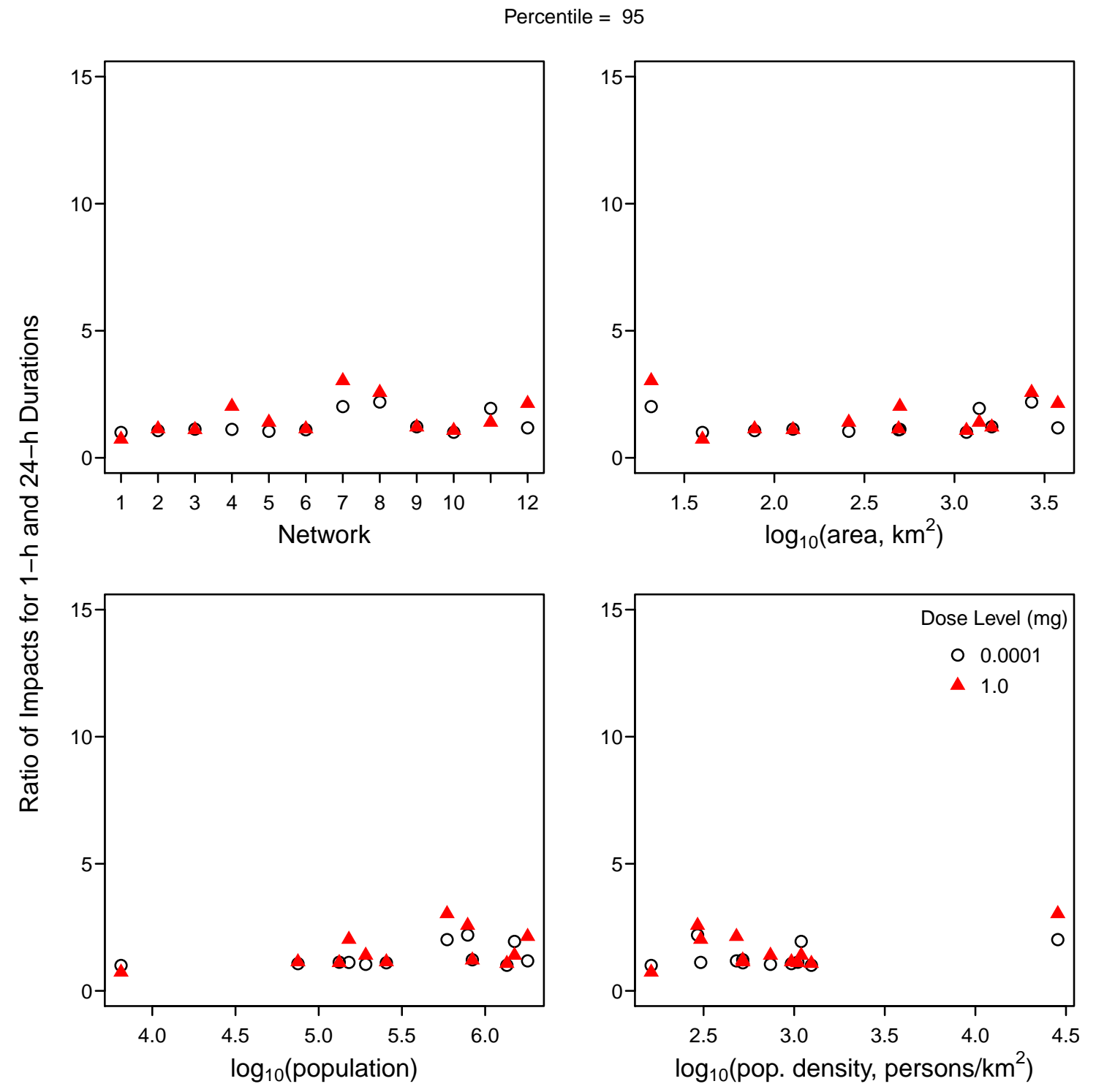

Figure 10.1. Summary of the ratio of $95^{\text {th }}$ percentile impacts associated with 1- and 24-h injections for the networks for two dose levels. Summaries are provided by network, by network area, network population, and average population density for each network. 


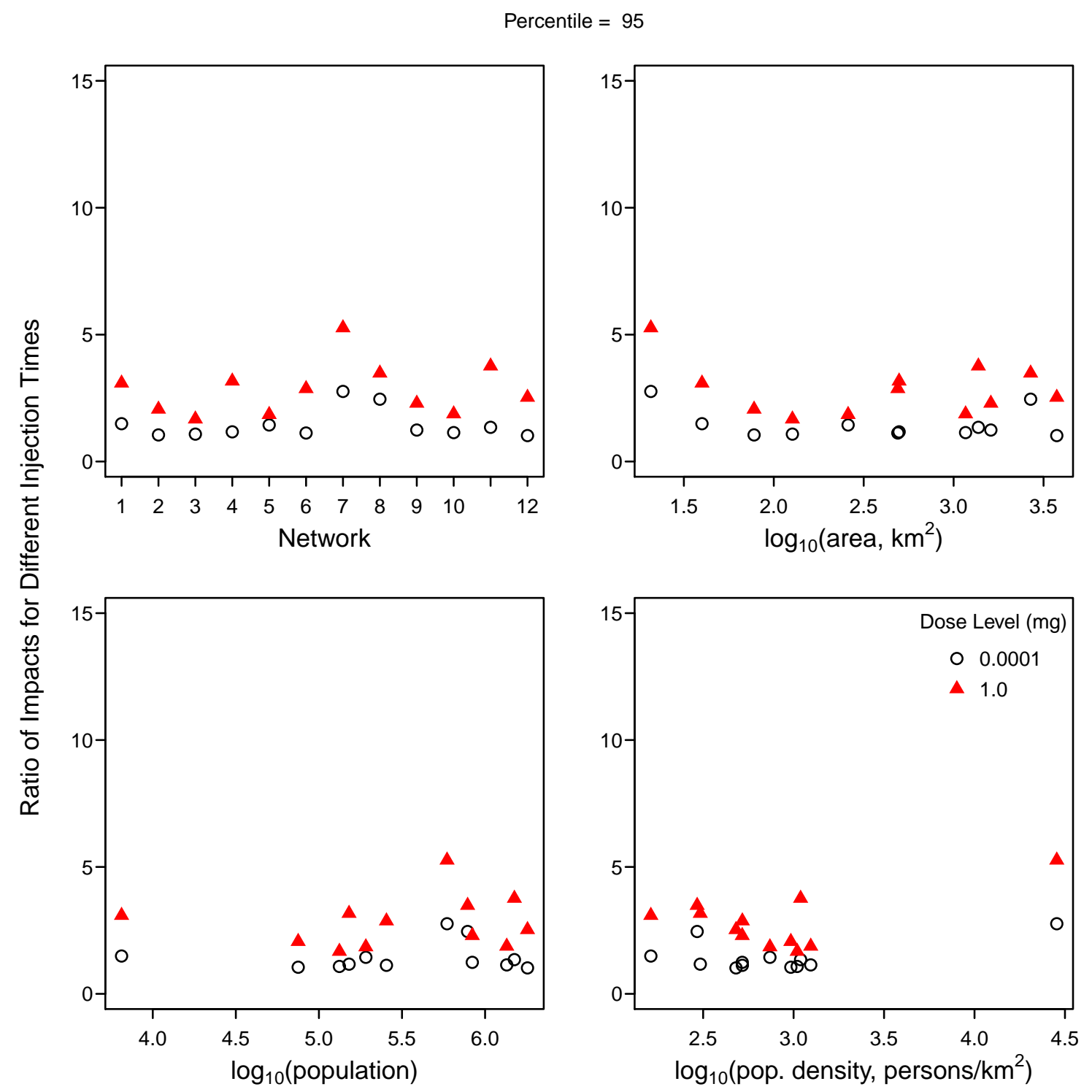

Figure 10.2. Summary of the maximum ratio of $95^{\text {th }}$ percentile impacts associated with different injection times (0:00, 6:00, 12:00, and 18:00) for the networks for two dose levels. Summaries are provided by network, by network area, network population, and average population density for each network. 
Percentile $=95$
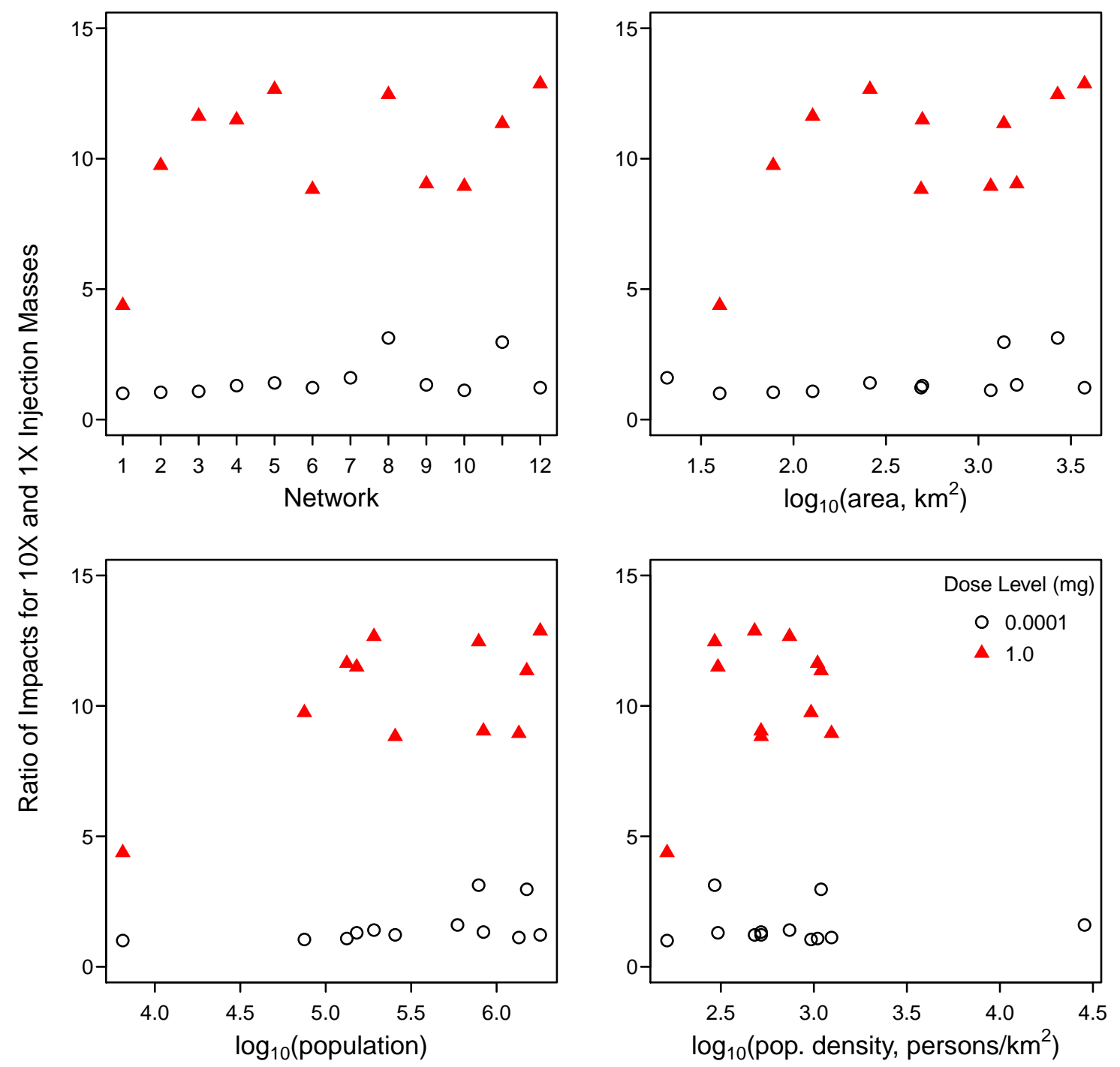

Figure 10.3. Summary of the ratio of $95^{\text {th }}$ percentile impacts associated with different injection masses (1X and 10X) for the networks for two dose levels. Summaries are provided by network, by network area, network population, and average population density for each network. No point is shown in the plots for Network 7 for a dose level of $1.0 \mathrm{mg}$ because the ratio of 21.3 is off the vertical scale. 


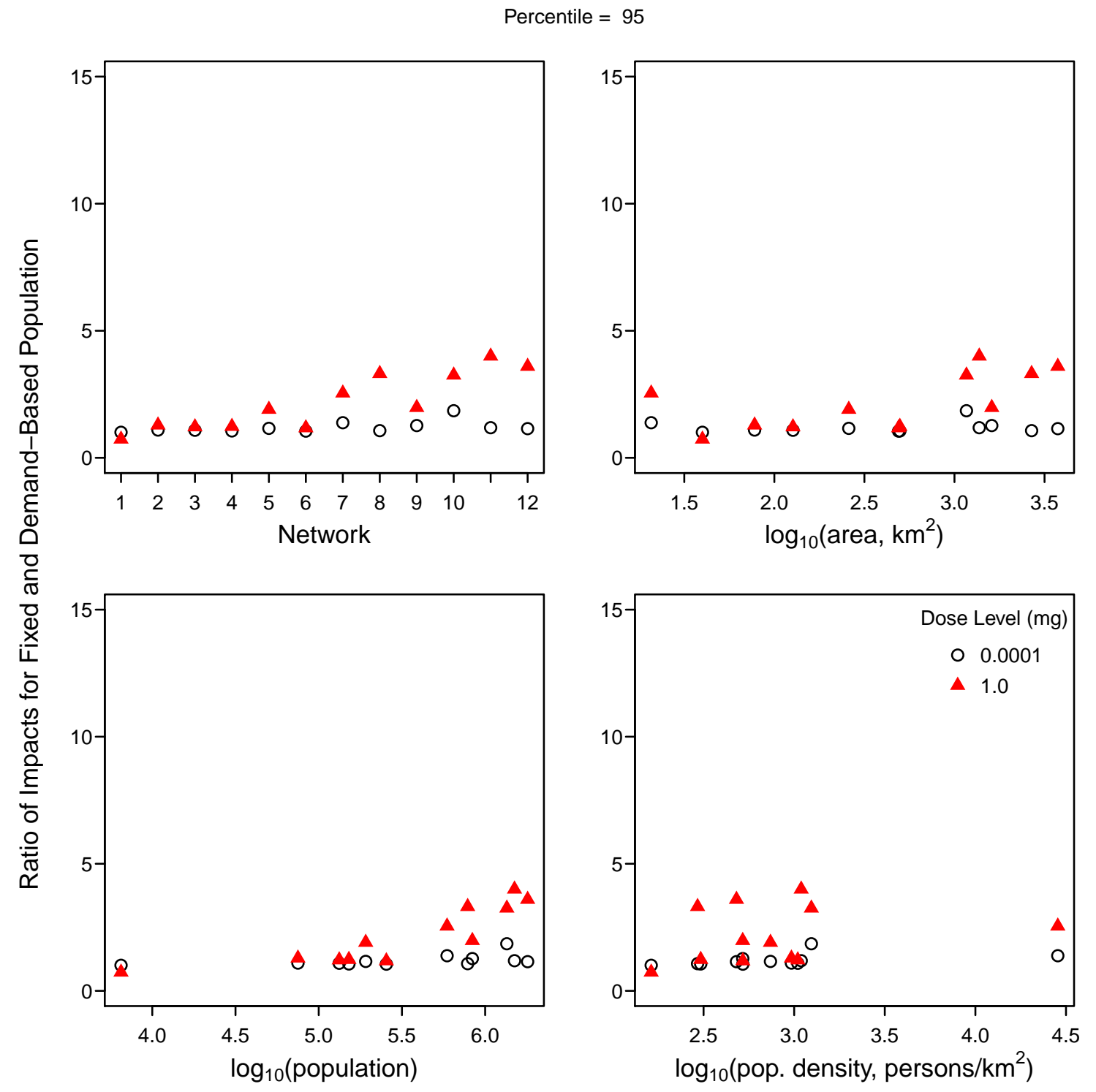

Figure 10.4. Summary of the ratio of $95^{\text {th }}$ percentile impacts associated with the two population models for the networks for two dose levels. Summaries are provided by network, by network area, network population, and average population density for each network. 


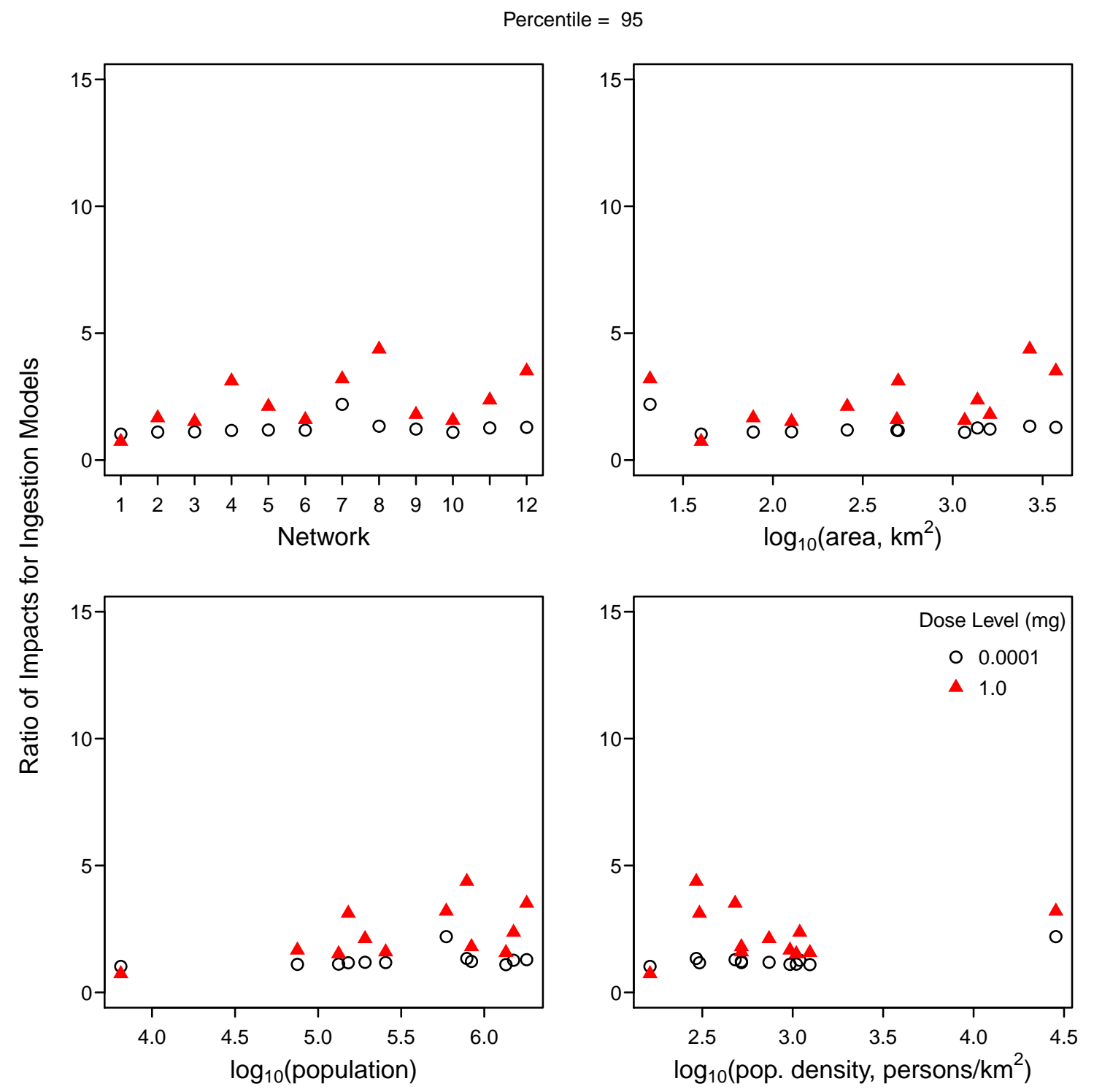

Figure 10.5. Summary of the ratio of $95^{\text {th }}$ percentile impacts associated with the two ingestion models for the networks for two dose levels. Summaries are provided by network, by network area, network population, and average population density for each network. 


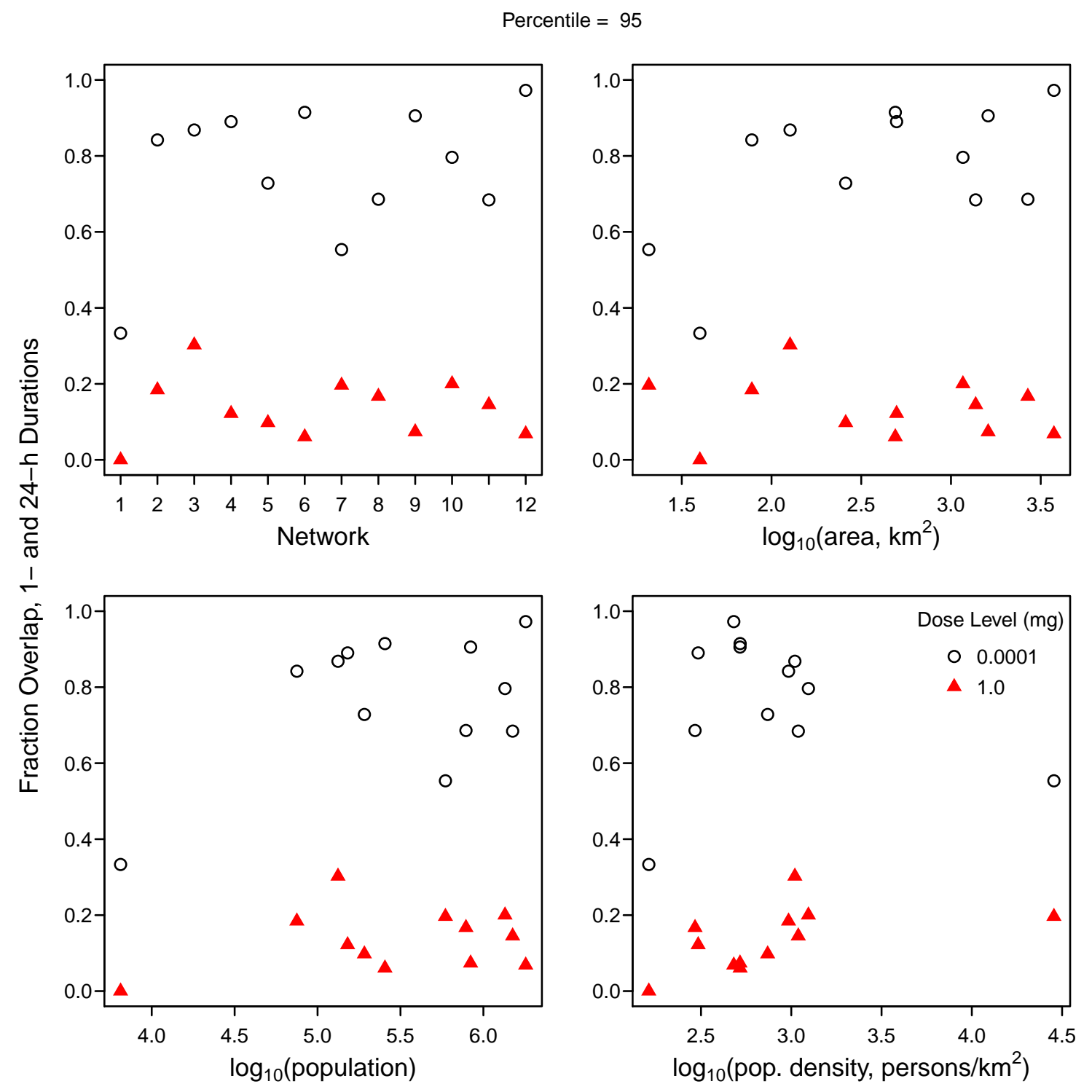

Figure 10.6. Summary of the overlap of $95^{\text {th }}$ percentile and higher injection nodes associated with 1-h and 24-h injections for the networks for two dose levels. Summaries are provided by network, by network area, network population, and average population density for each network. 

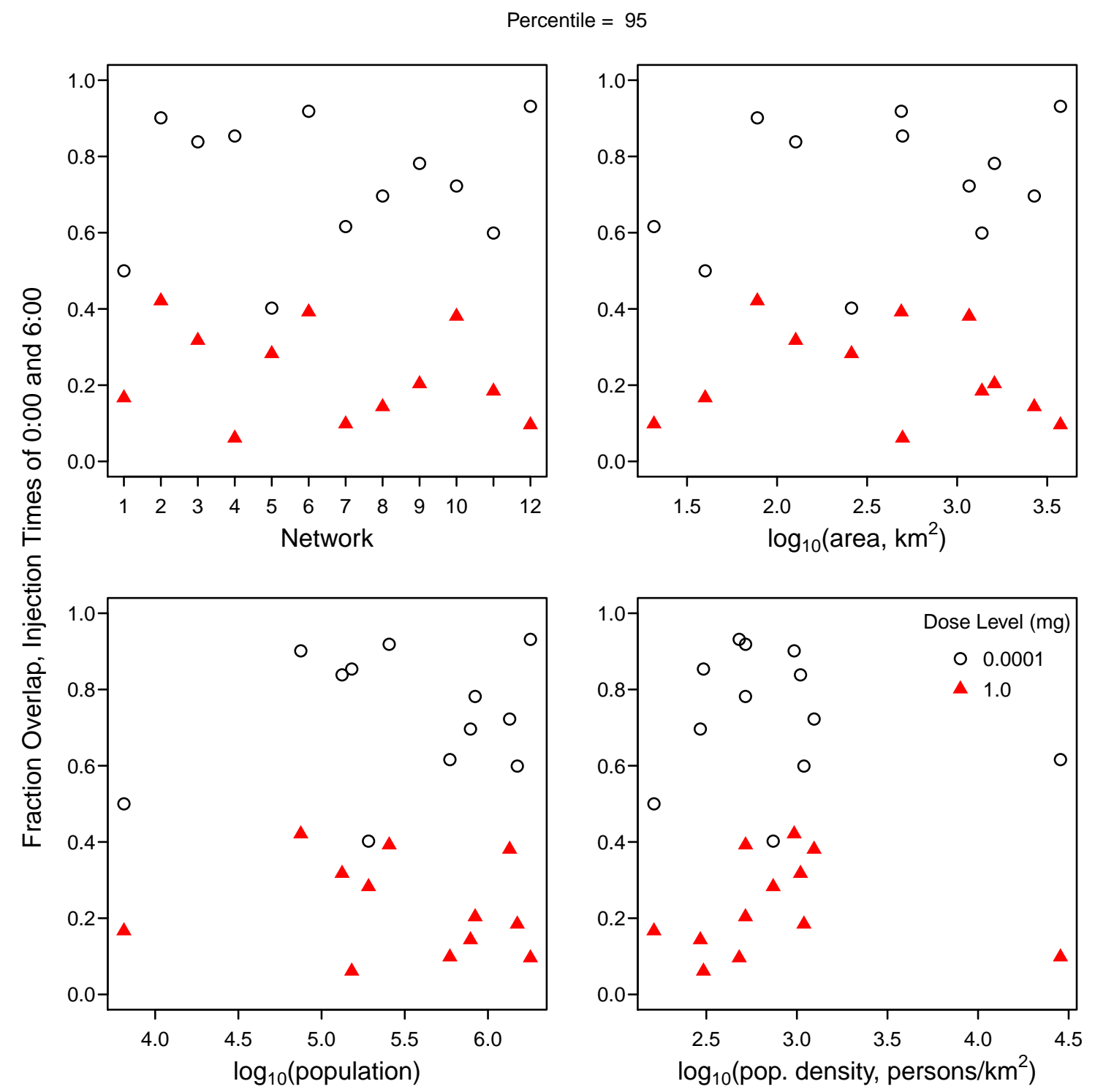

Figure 10.7. Summary of the overlap of $95^{\text {th }}$ percentile and higher injection nodes associated with injection times of 0:00 and 6:00 for the networks for two dose levels. Summaries are provided by network, by network area, network population, and average population density for each network. 


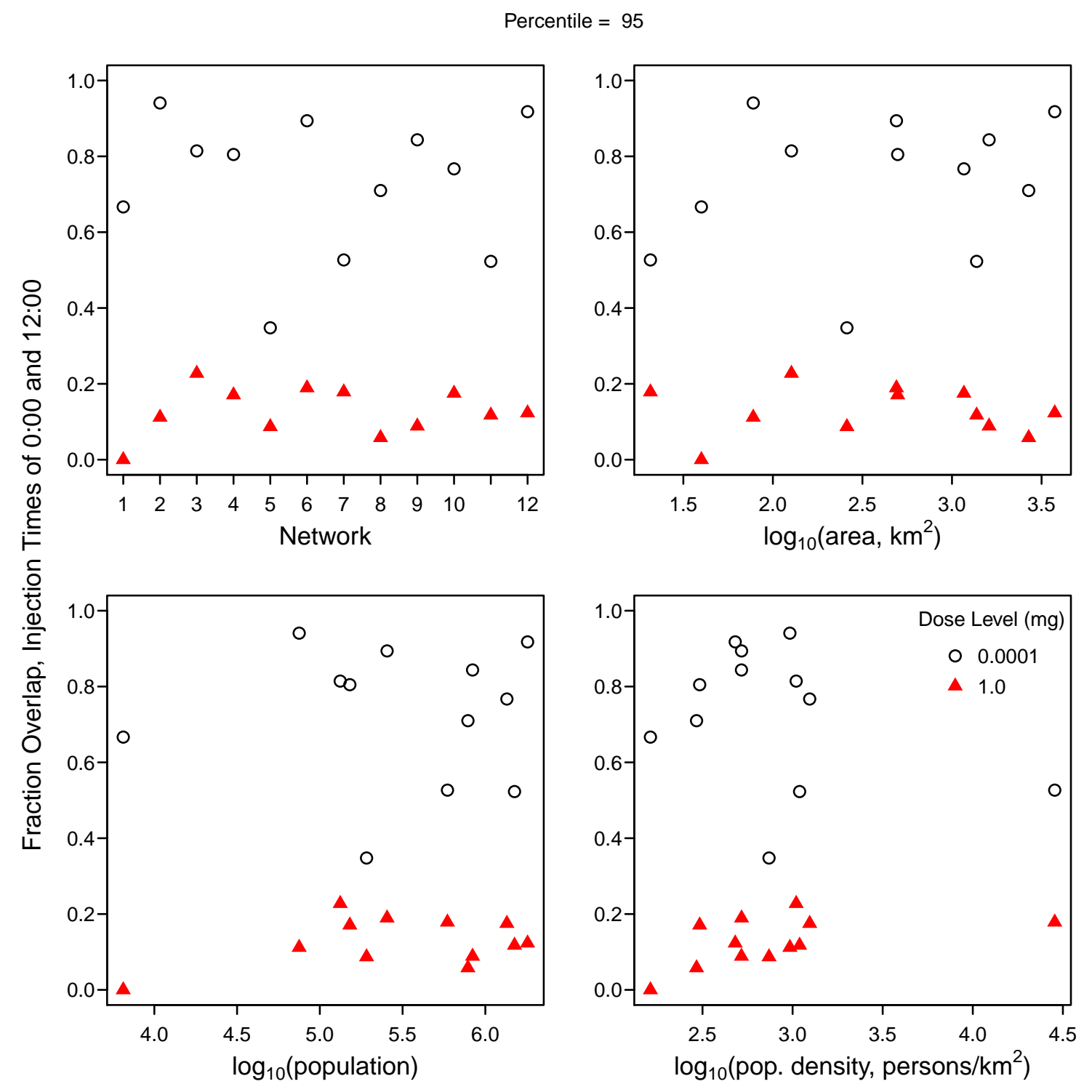

Figure 10.8. Summary of the overlap of $95^{\text {th }}$ percentile and higher injection nodes associated with injection times of 0:00 and 12:00 for the networks for two dose levels. Summaries are provided by network, by network area, network population, and average population density for each network. 


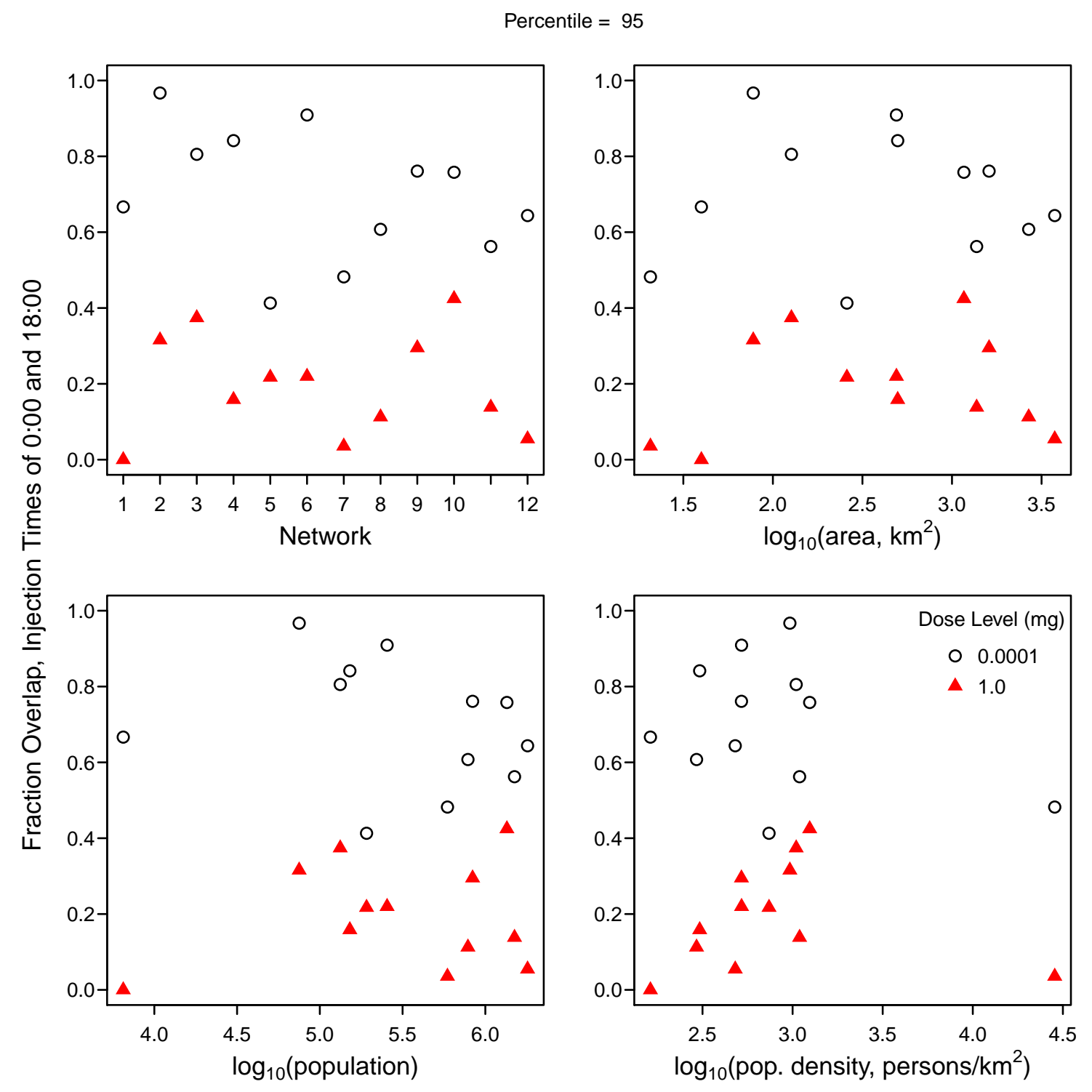

Figure 10.9. Summary of the overlap of $95^{\text {th }}$ percentile and higher injection nodes associated with injection times of 0:00 and 18:00 for the networks for two dose levels. Summaries are provided by network, by network area, network population, and average population density for each network. 


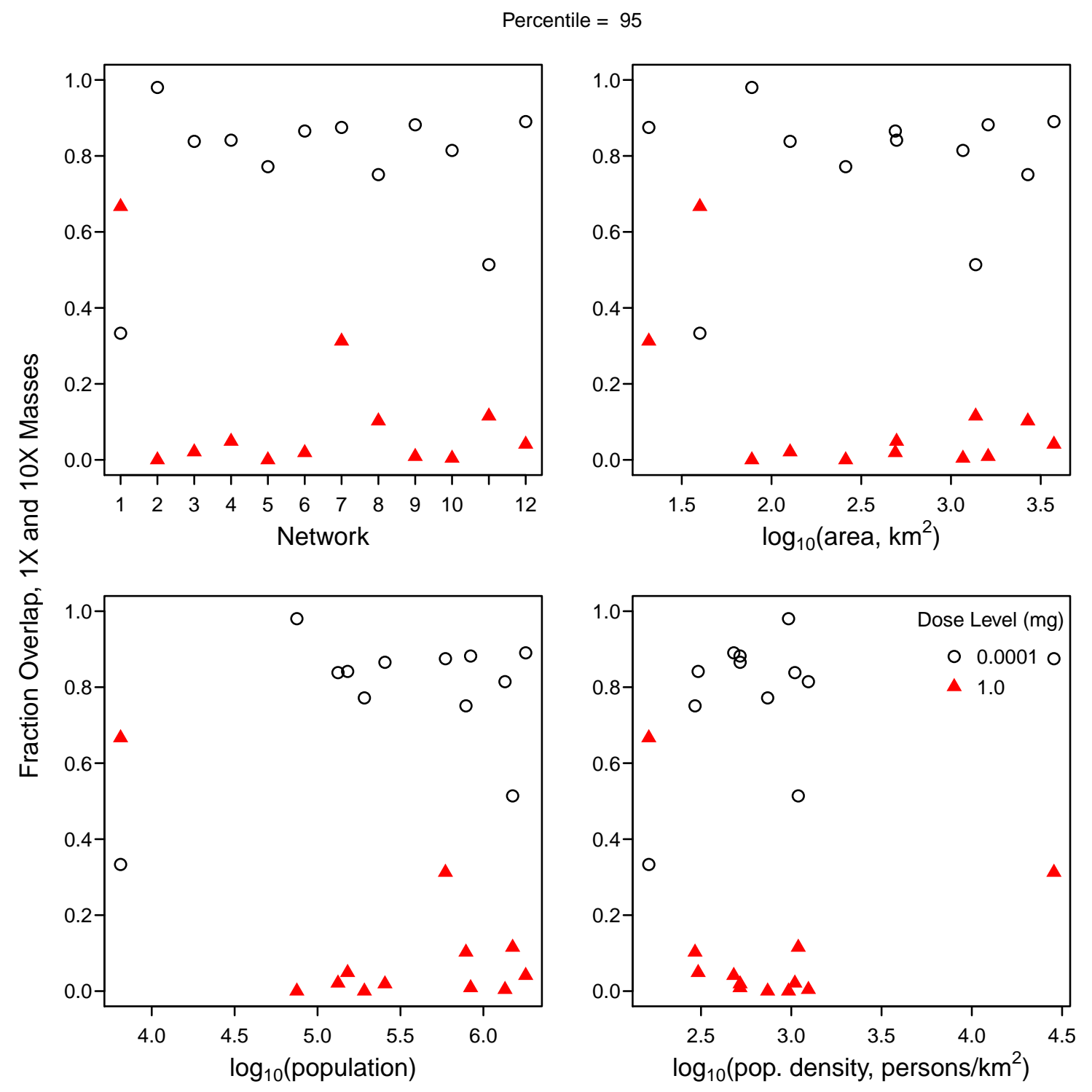

Figure 10.10. Summary of the overlap of $95^{\text {th }}$ percentile and higher injection nodes associated with $1 \mathrm{X}$ and 10X injection masses for the networks for two dose levels. Summaries are provided by network, by network area, network population, and average population density for each network. 


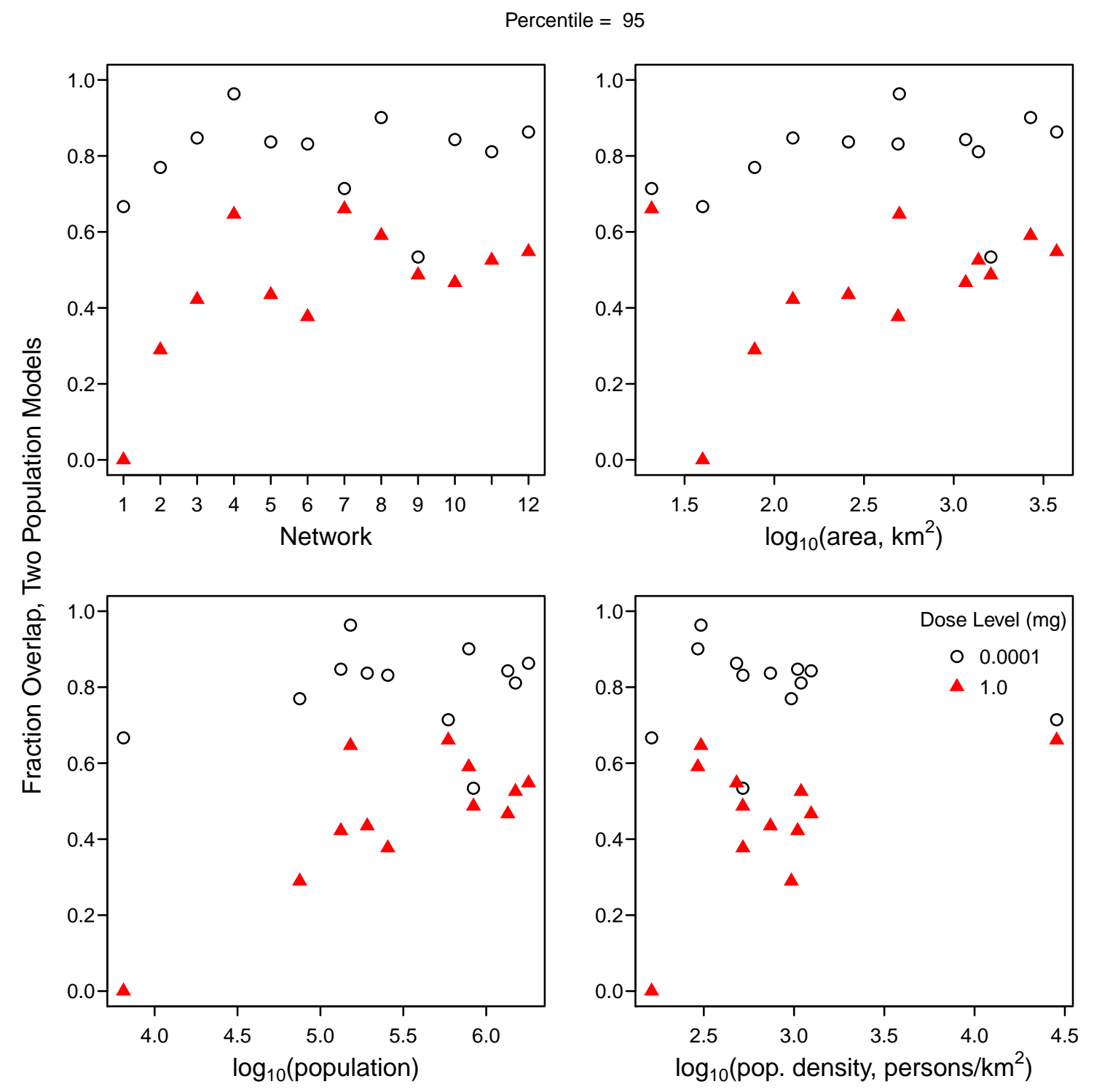

Figure 10.11. Summary of the overlap of $95^{\text {th }}$ percentile and higher injection nodes for the networks for the two population models for two dose levels. Summaries are provided by network, by network area, network population, and average population density for each network. 


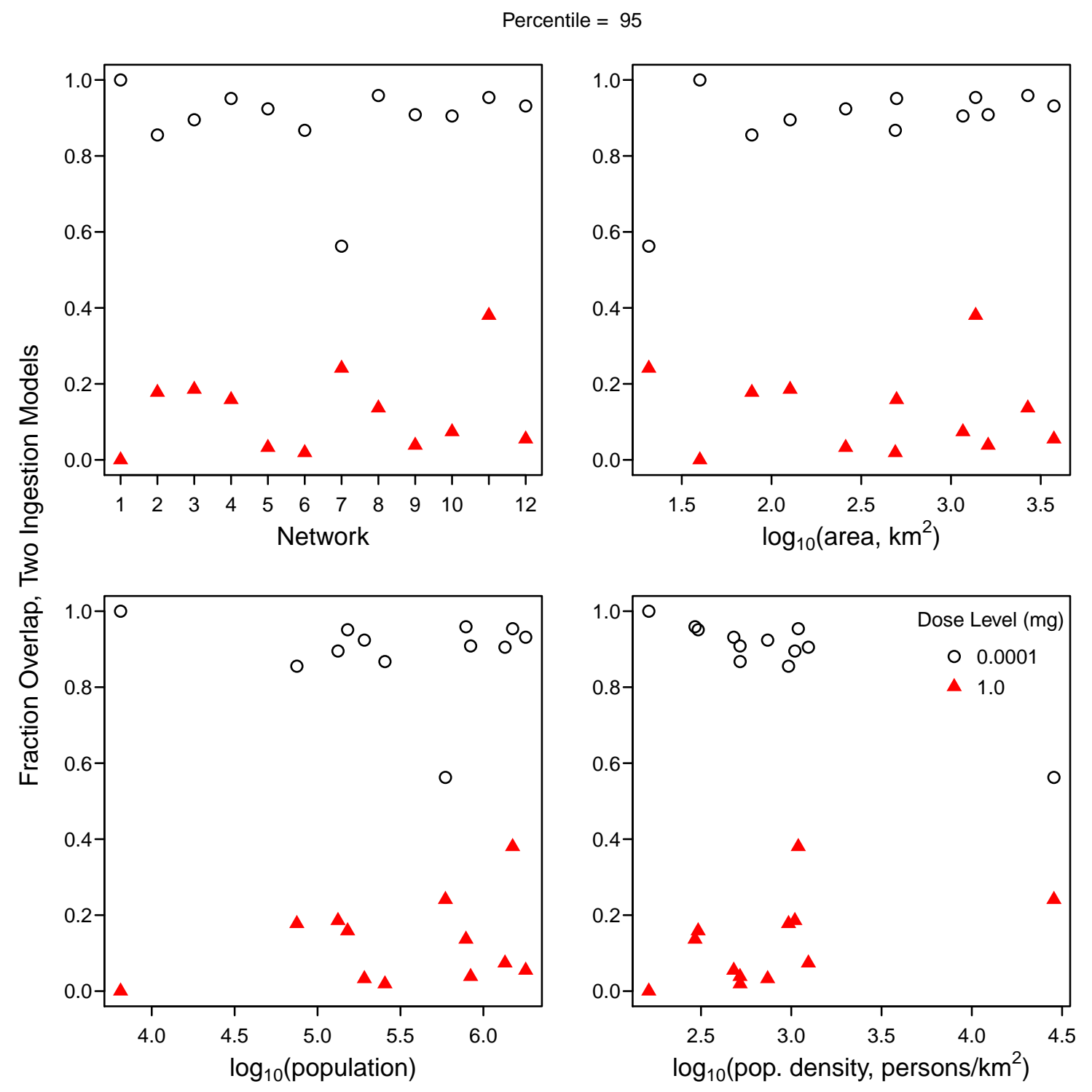

Figure 10.12. Summary of the overlap of $95^{\text {th }}$ percentile and higher injection nodes for the networks for the two ingestion models for two dose levels. Summaries are provided by network, by network area, network population, and average population density for each network. 


\section{Section 11}

\section{Discussion}

\subsection{Methodological Issues}

Importance of Dose Level. We have considered dose level as a parameter in our analysis of sensitivity. Instead of using dose level as a parameter, we could use median lethal dose as a factor with several levels, corresponding to different contaminants. Impacts are very sensitive to the value used for median lethal dose. As can be seen in Figures 3.1, 3.2, and 3.3, for $95^{\text {th }}$ percentile impacts, varying dose level from 0.0001 to $10 \mathrm{mg}$ results in impacts changing by over two to over five orders of magnitude, depending on the network. Figures 11.1 through 11.4 provide maps of Network 4 comparing the locations of high percentile injection nodes for dose levels of 0.001 , $0.01,0.1$, and $1.0 \mathrm{mg}$ with locations for a dose level of $0.0001 \mathrm{mg}$, using our baseline cases for factors. The locations of the injection nodes change greatly as the dose level increases. No overlap of the injection nodes occurs for dose levels of $0.0001 \mathrm{mg}$ and either $0.1 \mathrm{or} 1.0 \mathrm{mg}$.

Use of NZD Nodes as Injection Nodes. As noted above, we used NZD nodes as injection nodes because we believe that they represent the most likely injection locations. However, to assess the significance of our choice of injection nodes, we estimated impacts for a number of the networks using all nodes as potential injection nodes, in addition to considering only NZD nodes. We examined Networks 1, 4, 5, 7, and 12, which are the networks for which NZD nodes constitute the smallest fraction of all network nodes. We found that the results for impacts versus dose level (as illustrated by Figures 3.1, 3.2, and 3.3) obtained using all the nodes did not differ significantly from those obtained using only NZD nodes. (A quantitative comparison is provided in the supplemental material for Davis and Janke (2010).)

Injection at Terminal Nodes. Distribution system models consist of nodes with a demand and those with no demand. For the distribution system models examined here, demand nodes generally represent groups of service connections. Depending on the level of model detail, the number of service connections per node ranges from approximately 5 to 200. EPANET, and therefore also TEVA-SPOT, model contaminant injection as mass injected per unit time ( $\mathrm{mg} / \mathrm{min})$, with the assumption that any volume changes are insignificant. TEVA-SPOT does not have the ability to model volume additions to pressurized pipe flow. If a model has sufficient detail that all NZD nodes in a model represent terminal nodes in the network or individual service connections, people located at these nodes will only receive water from the distribution system but never release water 
back to the network. As a result, simulating an injection of a contaminant at these nodes in TEVASPOT will result in only people at the injection node being affected.

For example, if an injection of contaminant occurs at a terminal node at hour 0 , the entire mass of the injected contaminant is removed from the system within the hour and unless ingestion occurs within the hour that the contaminant is introduced no one is affected. For the hourly, demand-based timing model (used in Model 2), the number of people affected will equal the number of people at the node. For the probabilistic timing model (used in Model 1), however, only a fraction of the people at the node will be affected since not all individuals will ingest water during the hour that the contaminant was introduced.

An example of the issue involving terminal nodes occurs for the synthetic model named Micropolis (Brumbelow et al. 2007), but will occur with any model having nodes that only receive water. It is particularly evident with Micropolis because all the NZD nodes represent individual service connections or terminal nodes in the network, which only receive water. We modeled both the non-zero and zero demand nodes in Micropolis and found that, unlike the case for the 12 real systems, simulation of contamination events at the NZD nodes produced very small or near zero impacts for the various dose levels using ingestion Model 1. However, our simulation of contamination events at the zero demand nodes resulted in impacts generally consistent with the impacts obtained for the 12 real systems.

As a result, TEVA-SPOT simulations of contaminant injections at terminal nodes or nodes that only receive water will likely underpredict impacts for two reasons. First, not everyone at such nodes who would likely ingest contaminated water may be determined to be exposed due to removal of all contaminated water via demand at the node before ingestion occurs. Second, in the models, contaminants injected at terminal nodes have no means for conveyance back into the distribution system. Any ingestion of the contaminant occurs at the injection node.

The inability to properly determine impacts associated with injections at terminal nodes results in some uncertainty in our results, which will be network specific. The portion of NZD nodes in our 12 networks that are terminal nodes ranges from about $1 \%$ for Network 7 to about $27 \%$ for Network 3 . The median value is $12.8 \%$. Impacts associated with injections at terminal nodes tend to be underestimated and, therefore, impacts at some percentile levels for the networks also will tend to be underestimated. However, unless these nodes are located such that associated impacts are larger than would be expected for randomly located nodes, the magnitudes of high percentile impacts should not be much affected. The actual significance of any underestimate likely depends on the network, both because of the variation in the fraction of such nodes in the networks and their location. Quantifying the significance of any underestimate requires modifying the network models.

\subsection{Computational Issues}

Computational Effort. The analyses needed for this report required a large number of simulations and a large amount of computational time. Additionally, a considerable amount of data storage was needed for the processing of the simulations and analysis of results. The cases analyzed for the 12 networks required the simulation of over 1.6 million contamination events and occupied approximately 1.3 terabytes of storage. Archiving capabilities available within TEVASPOT significantly reduced the storage requirements. 
For a computer with a single processor (with approximately 3 gigahertz processor speed) and with 2 gigabytes of memory, TEVA-SPOT impact-analysis runtimes per simulation vary considerably for the 12 networks, from approximately 30 seconds (Network 1) to approximately 6 minutes (Networks 10 and 11). The more than 1.6 million simulations for the 12 networks would have taken approximately 100,000 hours (11 years) to complete on a single computer with one processor. Given the distributed computing capabilities utilized for this work (76 processors), total runtime was reduced considerably, to approximately 1,300 hours (54 days).

Expanding our analysis of the interaction of factors, particularly to use a Monte Carlo approach for all of the 12 networks, encounters computational difficulties. For Network 2, 32 combinations of factors were considered, requiring 32 separate simulations for each of the 3,000 NZD nodes in the network. For the other 11 networks, only 12 such sets of simulations were done. These simulations required about $60 \%$ of the processor time for this study, requiring a total runtime of about 30 days. Expanding these simulations to include all 32 combinations of factors for all 12 networks would therefore require about another 50 days of runtime. Addition of another factor doubles the required runtimes. To include a Monte Carlo approach for all of the networks could increase the total number of simulations by a factor of 1,000 or more, resulting in total runtimes of months or longer on all but very large distributed computing systems. Even for Network 2 alone, for which about 11 hours of runtime were required to examine the 32 combinations of factors considered, use of a Monte Carlo approach would require large runtimes on available computational resources.

Water Quality Tolerance Parameter. The EPANET water quality tolerance parameter specifies the smallest change in water quality that will result in a new parcel of water being created in a pipe. The default setting in EPANET is $0.01 \mathrm{mg} / \mathrm{L}$ for chemicals (Rossman 2000). The water quality tolerance determines whether the quality of one parcel of water is the same as that of another parcel. For a chemical contaminant the value could be the detection limit of the analytical method used to measure the chemical contaminant, adjusted by a suitable safety factor (Rossman 2000). Generally, lowering the water quality tolerance will provide increased accuracy of the water quality modeling results.

We examined the influence of the water quality tolerance value on estimated impacts for six networks $(1,2,4,5,7$, and 12$)$. Mean impacts and $50^{\text {th }}$ to $100^{\text {th }}$ percentile impacts were determined for each network using the default water quality tolerance value $(0.01 \mathrm{mg} / \mathrm{L})$ and using values of $10^{-6}$ and zero. For the lowest dose level, differences in impacts ranged from about $1 \%$ to $12 \%$, depending on the network and the percentile of the scenario. For higher dose levels, differences were generally $1 \%$ or less. For a few scenarios for high dose levels of $1 \mathrm{mg}$ or greater, we observed larger differences (20\% to $40 \%)$. A component of the differences observed is a result of the randomness in the impacts estimated using the probabilistic exposure model. Given the relative nature of the sensitivity results, these differences do not affect the conclusions reported.

The differences between the impacts obtained using water quality tolerance values of $10^{-6}$ and zero are generally much smaller than the differences between the default value and zero, indicating that better results can be obtained without using the more computationally burdensome value of zero for water quality tolerance. The water quality tolerance value selected should be aligned with the chemical contaminant being modeled and its associated mass release rate. 


\subsection{Application of Results}

Ultimately, the value of the sensitivity analysis presented here relates to its significance for the design of contamination warning systems and network protection. CWSs are designed to minimize in some way the adverse effects associated with a contamination event. Therefore, estimating such adverse effects is an important part of any CWS design. However, CWSs generally have not been designed with attention given to the importance of the various factors involved in estimating adverse effects. The sensitivity analysis presented here should alert designers of CWSs to the importance of (1) the various factors involved in defining a contamination event, (2) the possible uncertainties associated with establishing the distribution of the population within a network and estimating ingestion doses, (3) the dose level (or contaminant) used in determining impacts, and (4) network-to-network variability.

Although it is tempting to want to use results obtained with a single network model (or multiple models) as the basis for general conclusions about the response of water distribution systems to the intentional injection of contaminants, this study demonstrates that great variability in responses can occur for different systems. Although definite patterns exist in responses that extend across networks, as is shown here and in Davis and Janke (2010), substantial inter-network variablility limits the current ability to generalize quantitative results from a limited sample to new applications. Individual water distribution systems should be treated as unique systems with some similarities in the magnitudes and patterns of impacts during contamination events.

\subsection{Additional Work}

The work presented here could be expanded by including additional water distribution systems, other factors affecting the estimated impacts, and a Monte Carlo analysis of impacts for the combination of factors. The current analysis already includes a diverse group of systems. The major value of analyzing multiple systems is to demonstrate the importance of inter-network variability in the sensitivity to the factors studied. This variability is demonstrated by the group of 12 systems examined in this study. Unless additional systems having specific characteristics of interest are identified or the goal is to examine statistically how sensitivity depends on some system characteristic (e.g., use of skeletonization in the network model), the value of including additional systems is limited. Other factors are involved in the simulations used to obtain impacts that could be included in a sensitivity analysis. In particular, we did not consider explicitly any factors related to the network model or to the approach used to determine flows and water quality in the networks. (We did check for possible effects of network population, area, and population density on our results and found no apparent influence.) The emphasis here is on the nature of the injection and the exposure process. As noted above, use of a Monte Carlo approach to examining interactions among factors encounters computational difficulties for the large networks included in this study. However, as computational abilities improve, use of such an approach may become practical.

Two related areas where additional work is needed are the expansion of the analysis of impacts to include the effects of contaminant decay or removal in the network and the sensitivity of the design of CWSs to the various factors influencing estimated impacts. Decreases in contaminant concentrations due to processes other than dilution could have a significant effect on estimated impacts and the sensitivity of estimated impacts to such processes should be considered. CWS designs could be significantly affected by variations in the factors considered here and by the 
nature of the contaminant, as well as by any nonconservative behavior of the contaminant in the water distribution system. CWSs may perform differently if challenged by contaminants with low or high median lethal doses, for example. We are currently pursuing additional work in these two areas. 


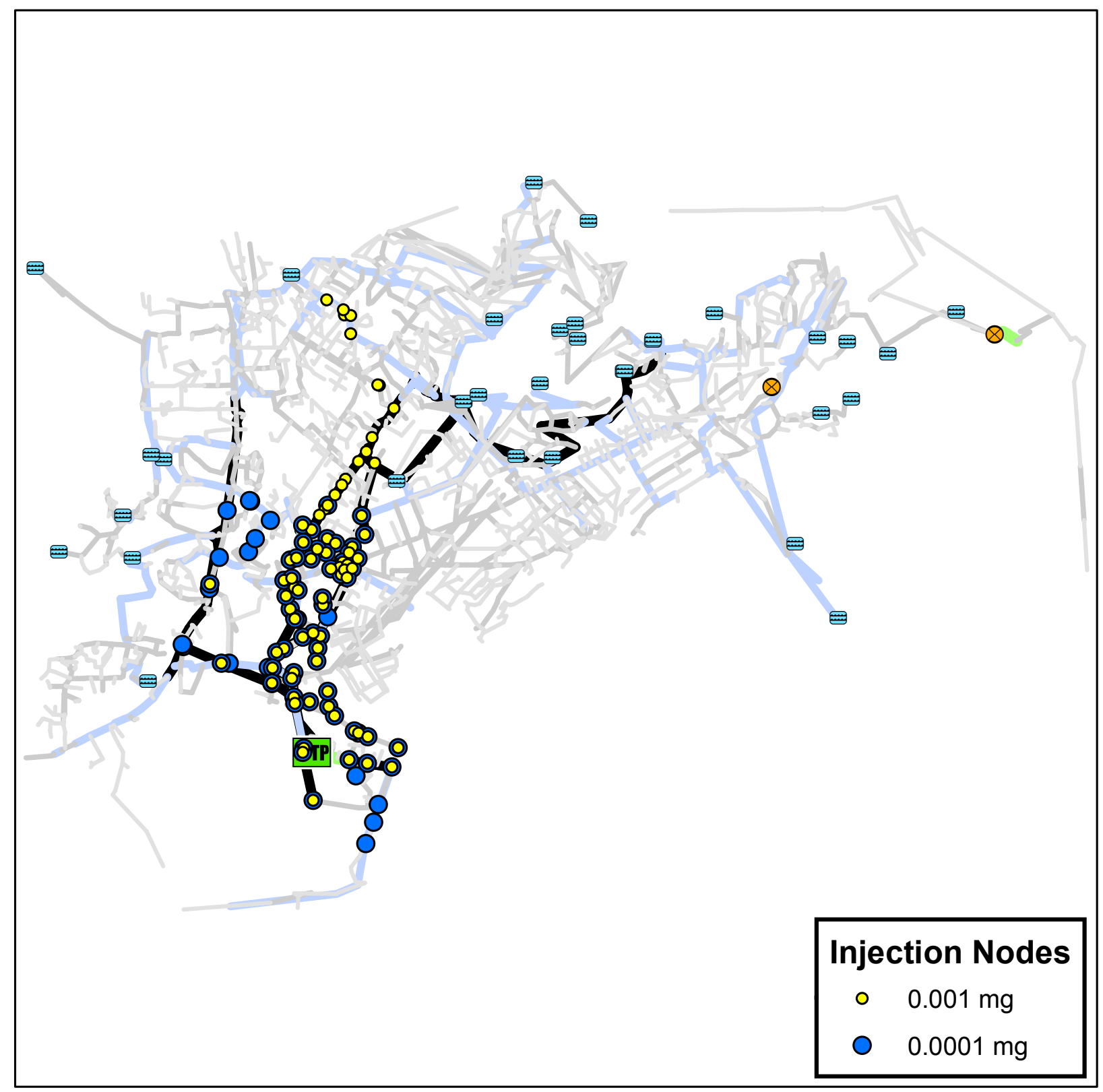

Figure 11.1. Network 4 showing locations of injection nodes associated with $95^{\text {th }}$ percentile or higher impacts at dose levels of $0.0001 \mathrm{mg}$ and $0.001 \mathrm{mg}$. The baseline case was used for all factors. 


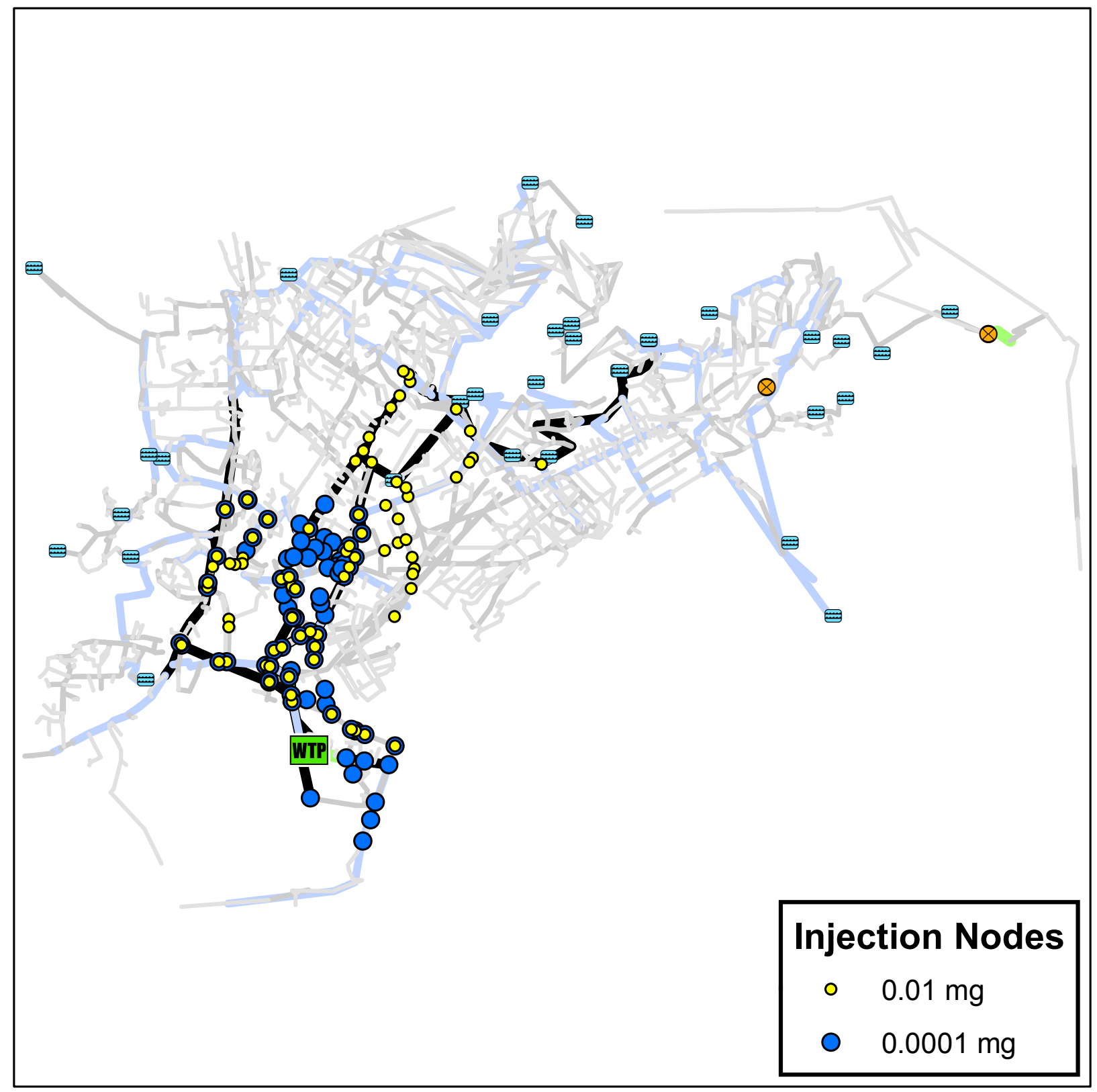

Figure 11.2. Network 4 showing locations of injection nodes associated with $95^{\text {th }}$ percentile or higher impacts at dose levels of $0.0001 \mathrm{mg}$ and $0.01 \mathrm{mg}$. The baseline case was used for all factors. 


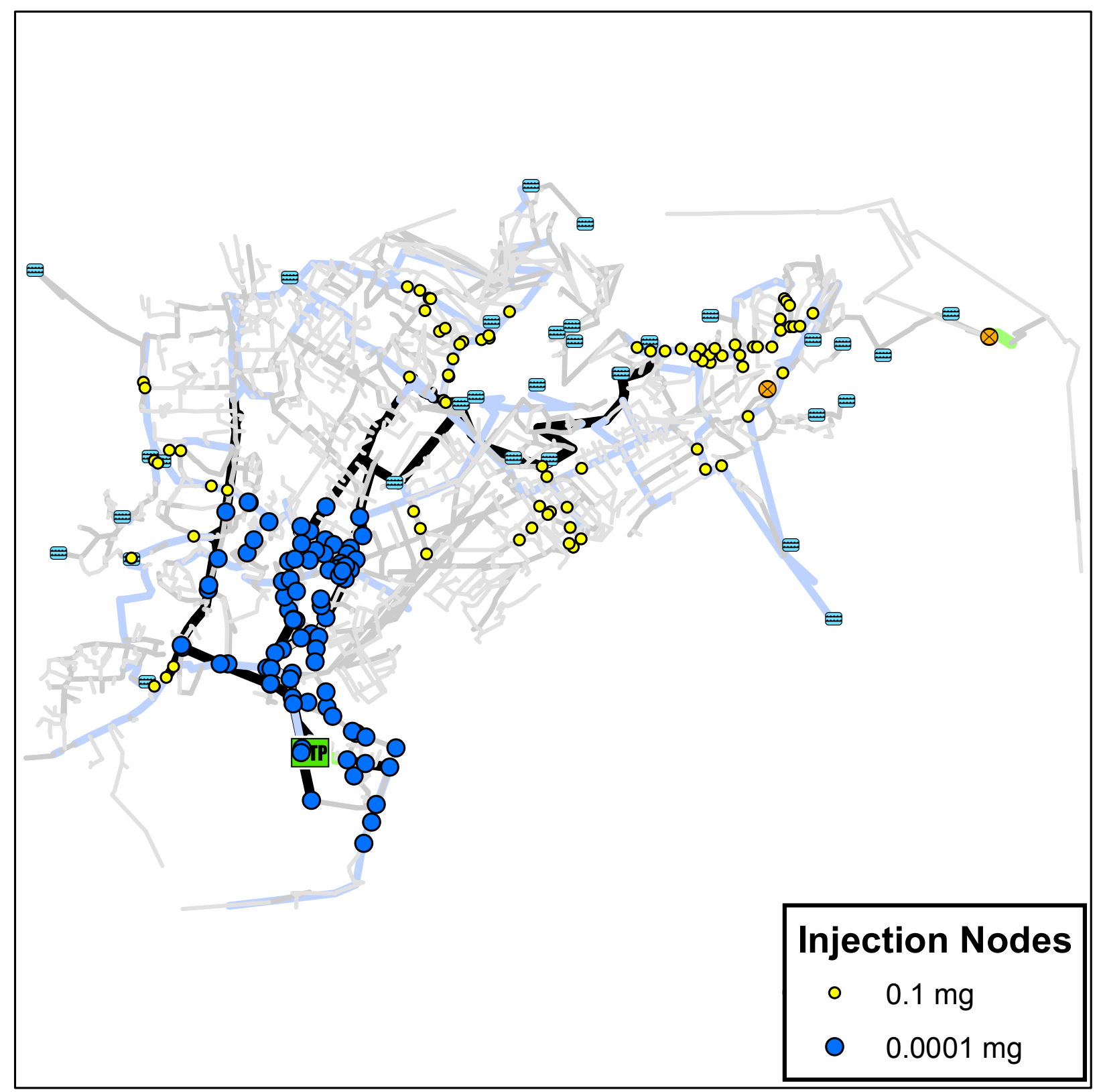

Figure 11.3. Network 4 showing locations of injection nodes associated with $95^{\text {th }}$ percentile or higher impacts at dose levels of $0.0001 \mathrm{mg}$ and $0.1 \mathrm{mg}$. The baseline case was used for all factors. 


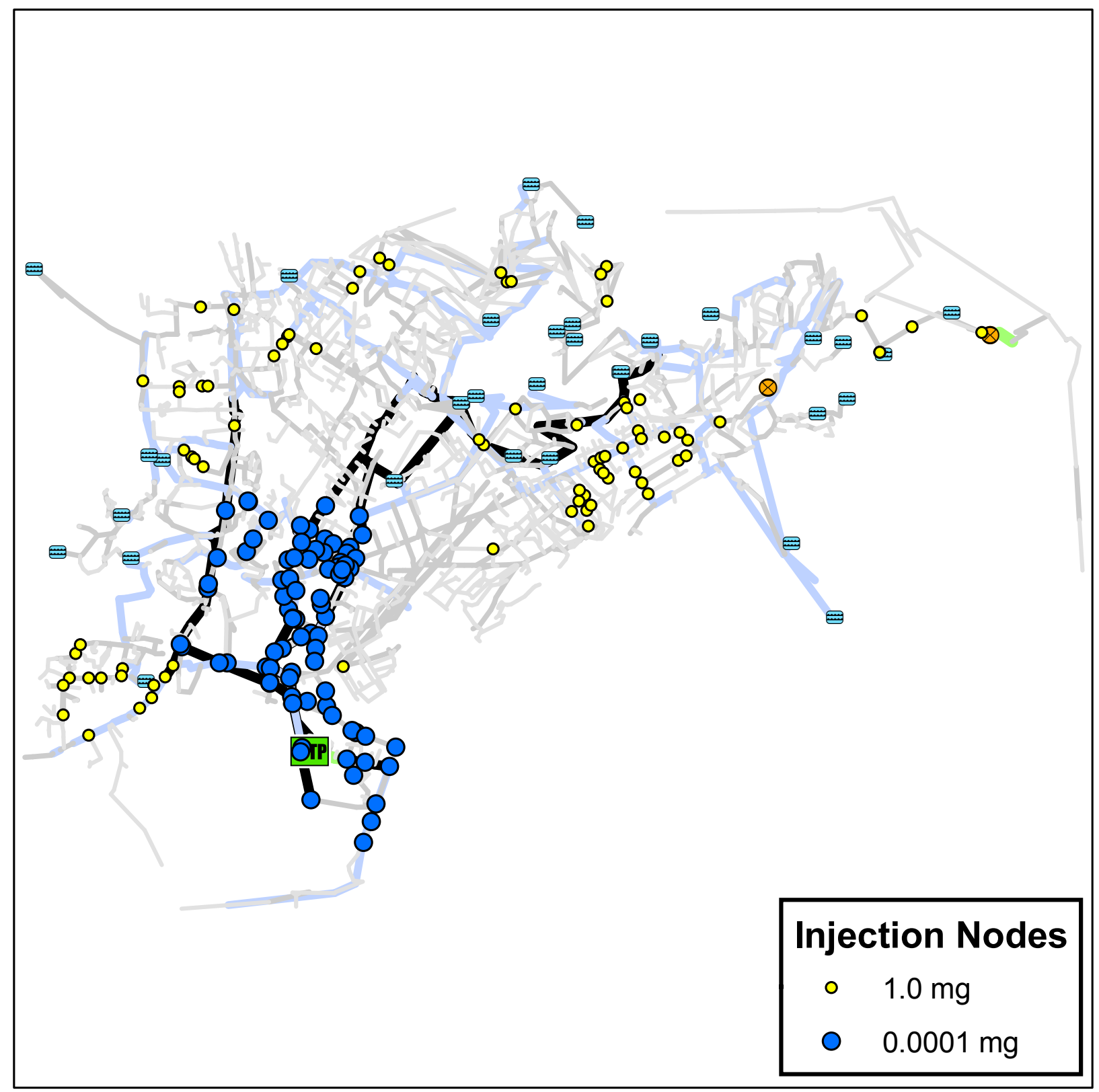

Figure 11.4. Network 4 showing locations of injection nodes associated with $95^{\text {th }}$ percentile or higher impacts at dose levels of $0.0001 \mathrm{mg}$ and $1 \mathrm{mg}$. The baseline case was used for all factors. 


\section{Section 12}

\section{Conclusions}

This sensitivity analysis considered five important factors related to assessing potential impacts associated with contamination events in water distribution systems: injection duration, injection time, injection mass, the distribution of population in the network, and the ingestion model. Estimated impacts are sensitive to all these factors, in varying amounts that depend on the network. When the factors are considered together (not including the ingestion model), depending on the dose level, the magnitudes of impacts are most sensitive to injection mass or duration:

- At low dose levels, impacts are most sensitive to injection duration, although the relative changes in impacts due to changes in duration may not be large for high percentile impacts. Impacts are larger for longer duration injections and the increases tend to be more important for lower percentile impacts.

- At low dose levels, impacts are not particularly sensitive to injection mass, given a likely range in injection masses.

- At higher dose levels, impacts are most sensitive to injection mass, with impacts increasing for larger injection mass.

The network-to-network variability in the sensitivity to the various factors considered here can be large. We did not find a correlation between any network-related feature and the sensitivities observed for the various networks. However, we examined only a small number of such features.

The influence of the various factors on the location of high percentile injection nodes can be as important or more important than their influences on the magnitudes of impacts. In addition, dose level has a major influence on the location of high percentile injection nodes.

The sensitivity of the location of high percentile injection nodes to the various factors is greatest at high dose levels.

Injection time, duration, and mass, as well as uncertainties in the distribution of population in a network and in the model used to estimate ingestion dose can all have a significant influence on estimated impacts for a contamination event. Designers of contamination warning systems need to consider the possible influences of all of these factors on their results, as well as the importance of network-to-network variability and the significance of the dose level or contaminant being used. 


\section{References}

Brumbelow, K., Torres, J., Guikema, S., Bristow, E., and Kanta, L. (2007). "Virtual cities for water distribution and infrastructure system research." Proceedings of the World Environmental and Water Resources Congress 2007, American Society of Civil Engineers. Tampa, Florida. DOI:10.1061/40927(243)469.

Davis, M. J., and Janke, R. (2008). "Importance of exposure model in estimating impacts when a water distribution system is contaminated." Journal of Water Resources Planning and Management, 134(5), 449-456.

Davis, M. J., and Janke, R. (2010). "Patterns in potential impacts associated with contamination events in water distribution systems." Journal of Water Resources Planning and Management, (16 March 2010), 10.1061/(ASCE)WR.1943-5452.0000084

Hintze, J. L., and Nelson, R. D. (1998). "Violin plots: a box plot-density trace synergism." The American Statistician, 52(2), 181-184, May.

Isovitsch, S.E., and VanBriesen, J.M. (2008). "Sensor placement and optimization criteria dependencies in a water distribution system." Journal of Water Resources Planning and Management, 134(2), 186-196.

Khanal, N. (2005). Generalized sensitivity analysis of water distribution system vulnerability to deliberate intrusions, Master of Science thesis, University of Cincinnati, Cincinnati, Ohio.

Khanal, N., Buchberger, S. G., and McKenna, S. A. (2006). "Distribution system contamination events: exposure, influence, and sensitivity." Journal of Water Resources Planning and Management, 132(4), 283-292.

LaTeX project (2010). "LaTeX - a document preparation system." http://www . latex-project.org/

Morley, K., Janke, R., Murray, R., and Fox, K. (2007). "Drinking water contamination warning systems: water utilities driving water security research." Journal of the American Water Works Association, 99(6), 40-46.

Murray, R.E., Grayman, W.M., Savic, D.A. and Farmani, R. (2009). "Effects of DMA redesign on water distribution system performance." Integrating Water Systems, Boxall, J. and Maksimovic, C. (eds.), Taylor and Francis, London, pp. 645-650. 
Nilsson, K. A., Buchberger, S. G., and Clark, R. M. (2005). "Simulating exposures to deliberate intrusions into water distribution systems." Journal of Water Resources Planning and Management, 131(3), 228-236.

Ostfeld, A., et al. (2008). "The Battle of the Water Sensor Networks (BWSN): A design challenge for engineers and algorithms." Journal of Water Resources Planning and Management, 134(6), 556-568.

R Development Core Team (2010). "R: A language and environment for statistical computing." R Foundation for Statistical Computing, Vienna, Austria. http://www.R-project.org (Feb. 21, 2010).

Rossman, L. A. (2000). “EPANET 2 users manual,” EPA/600/R-00/057, U.S. Environmental Protection Agency, National Risk Management Research Laboratory, Office of Research and Development, Cincinnati, Ohio.

U. S. EPA (U. S. Environmental Protection Agency). (2000a). "Estimated per capita water ingestion in the United States," EPA-822-00-008, Office of Water, Washington, D.C.

U. S. EPA. (2000b). "Policy and program requirements for the mandatory agency-wide quality system." EPA Order CIO 2105.0. Available at http://www.epa.gov/irmpoli8/ policies/21050.pdf

U. S. EPA. (2000c). "EPA quality manual for environmental programs." CIO 2105-P-01-0. Available at http://www.epa.gov/irmpoli8/policies/2105p010.pdf

U. S. EPA. (2010a). “Threat ensemble vulnerability assessment research program.” http:// www . epa.gov/nhsrc/water/teva.html (April 15, 2010).

U. S. EPA. (2010b). "Policy and Guidance: Guide to Requirements for Research Reports Submitted to the National Homeland Security Research Center." http: / /www.epa.gov/nhsrc/ guidance. html (April 26, 2010).

U. S. EPA. (2010c). 'Threat Ensemble Vulnerability Analysis - Sensor Placement Optimization Tool (TEVA-SPOT) Graphical User Interface Users Manual (2010)," version 2.2.0 BETA, EPA 600/R-08/147, U.S. Environmental Protection Agency, Office of Research and Development, National Homeland Security Research Center, Water Infrastructure Protection Division, Cincinnati, Ohio. Available at http://www.epa.gov/nhsrc/pubs/600r08147.pdf

Watson, J-P., Murray, R., and Hart, W. E. (2009). "Formulation and optimization of robust sensor placement problems for drinking water contamination warning systems." Journal of Infrastructure Systems, 15(4), 330-339. 


\section{Appendix: Quality Assurance}

The primary objective of the United States Environmental Protection Agency (EPA) Quality Assurance requirements is to ensure that all environmental data assembled, models and methodologies developed, and analytical results obtained are of sufficient quantity and quality to support the conclusions and observations produced. The EPA Quality System is defined by EPA Order CIO 2105.0 (U. S. EPA 2000b) and is described in the EPA Quality Manual for Environmental Programs (U. S. EPA 2000c). Quality assurance (QA) is the planned and systematic processes implemented to provide confidence in the results produced and the suitability of those results. Quality control (QC) is defined by the procedures implemented to ensure that the results and conclusions adhere to the defined set of quality criteria.

We implemented quality assurance and quality control methodologies and procedures at each step of our research project to ensure that the results produced are based on state-of-the art science and engineering principles, are reproducible, and suitable for estimating adverse public health effects associated with water distributions systems given an intentional contamination event.

All software development, research, model and algorithm development, data collection and analyses, and report writing were performed by the authors. QA/QC practices were implemented at each of the following phases of the research and report preparation:

- Research study (purpose and goals)

- Algorithmic and methodological model development

- Software engineering

- Model and software verification

- Computational modeling and simulation

- Data analysis and report preparation

In following paragraphs, we discuss the QA/QC practices implemented in each of these phases.

Research Study (purpose and goals.) The proper design of CWSs for water distribution systems depends on a thorough understanding of how adverse public health effects associated with contamination events are influenced by the major factors that define a contamination event. We conducted a literature review and found no information available for quantifying the sensitivity of estimated adverse health effects to the major factors defining intentional contamination events for a range of real water distribution systems. We summarize our findings of the literature study in the introduction. Given the lack of such information, we formulated a research plan, the technical 
portion of which is documented in this report. This plan was used to investigate the sensitivity of adverse effects associated with an intentional contamination event to the major factors defining the event and the major assumptions made in assessing exposure to the contaminant. In developing the research plan, we fully described the purpose and goals of the study. Separately, we identified potential customers and stakeholders (water utility partners, water industry consultants, American Water Works Association, EPA Office of Water, Department of Homeland Security, and Department of Defense (military installations) who would likely find the research results and findings useful (Morley et al. 2007). Our approach to the study relied on established software to conduct simulations and established science and engineering principles.

Algorithmic and Methodological Model Development. We relied on the established EPANET (Rossman 2000) and TEVA-SPOT (U. S. EPA 2010a) software programs. We used only the most up-to-date versions of the programs for the results included in this report. Our set of real and operationally valid water distribution system models were obtained from collaborating water utilities. Our approach for assessing adverse effects was previously developed and described (Davis and Janke 2008, 2010). Our exposure models for the timing of ingestion of contaminated tap water were previously developed and described ((Davis and Janke 2008). Model input parameters were specified in each water system 's network model. Although network model parameters were not independently verified, each model represents a real, operationally valid system. Three of the water system models utilized have previously been reported on in peer-reviewed publications. Our data analysis, including all statistical analyses, relied on established methods and procedures, with all such procedures fully described and referenced in this report.

Software Engineering. TEVA-SPOT is a stand-alone program developed to study contamination vulnerability assessment and sensor network design for water distribution systems. TEVA-SPOT was developed based on EPANET and is maintained to be consistent with the latest version of EPANET. EPANET (version 2.00.12) is integrated into TEVA-SPOT through the EPANET Programmer's Toolbox application. Several important differences exist between the execution of EPANET within TEVA-SPOT as opposed to the execution of EPANET through its stand-alone graphical user interface. These differences, important to the assessment of exposure and risk, include: (1) TEVA-SPOT's ability to average contaminant water quality concentration data over the reporting interval and (2) TEVA-SPOT's use of the STEP Q function instead of NEXT Q function. Details on these EPANET functions are discussed in the EPANET users manual (Rossman 2000).

A mass balance feature was incorporated into TEVA-SPOT to allow the tracking of contaminant mass, i.e., contaminant mass injected, mass removed, mass in pipes and mass in tanks, in order to help verify the estimated impacts for reasonableness. The mass balance tracking feature supported the selection of a 1-minute water quality time step. Early simulations using a 5- minute water quality time step resulted in mass balance discrepancies.

Updates to TEVA-SPOT are independently verified with hand or spreadsheet calculations. All of our simulations were implemented on a distributed high performance computing system that includes 38 dual processor compute nodes for a total of 76 processors. TEVA-SPOT is openly distributed by request through the TEVA Research Program web site (U. S. EPA 2010a).

We maintain a subversion repository for all TEVA-SPOT and EPANET computer code. Our subversion repository tracks all changes made to the software by person, date, and time and allows for regression to earlier versions if needed. 
Model and Software Verification. Our water utility models were provided by partnering water utilities. No independent verification of model input parameters was performed. Models were run in TEVA-SPOT using the input parameters specified in the models. Network distribution system models were run for water-age analysis to help determine appropriate simulation runtimes. Models obtained from partnering water utilities met the requirements specified in the TEVA-SPOT Users Manual, Appendix A, "Water Utility Requirements for Using EPA's TEVA-SPOT Software" (U. S. EPA 2010c). Input parameters for TEVA-SPOT were developed consistent with the approach described in Section 2. Contamination scenarios for the 12 networks were defined consistent with Section 2 and implemented in TEVA-SPOT on a network-by-network basis. Collections of similar scenarios were designated as an ensemble and run in TEVA-SPOT. Each ensemble was uniquely named for easy recognition and verification of input parameters.

Using the ensemble naming structure, we routinely verified input parameters to ensure consistency with the network model (e.g., Network 1 versus Network 2) and required input parameters, e.g., population and ingestion model. Each executed TEVA-SPOT ensemble produced a uniquely named output file of dose-over-threshold results.

Our maps were created by exporting health impacts assessment results as Database IV (dBase ${ }^{\circledR}$ IV) data files from TEVA-SPOT and then merging and mapping the results in ArcGIS ${ }^{\circledR}$ 9.3.

We regularly backed up output consistent with EPA information technology and information management requirements. Additionally, we regularly archived dose-over-threshold data files to a database. We used these data files for verification of the consistency of dose-over-threshold results following updates to TEVA-SPOT.

Computational Modeling and Simulation. Given the large number of TEVA-SPOT ensembles executed, verification of input parameters for all simulations was a significant requirement and was routinely performed. When input errors or problems were found, the ensemble(s) was re-run and the analyses were updated with the new results. Results from early runs were compared with results from later runs to ensure consistency of results following any modifications of software.

The structure of the TEVA-SPOT software facilitated best management QA/QC practices:

- The database structure of TEVA-SPOT output provides organization and management of ensembles for easy data retrieval and analysis.

- TEVA-SPOT provides management of ensembles by providing date status information on each ensemble, e.g., (1) date the ensemble was last saved, (2) date ensemble was executed last, (3) date that health impacts assessment was executed last, and (4) size of the ensemble.

- Each dose-over-threshold output file is uniquely and automatically named by TEVA-SPOT to link to its parent ensemble name.

- Each ensemble executed in TEVA-SPOT is documented with an output of the network model used in the simulation, which was then used for QA/QC purposes to verify that the appropriate model was used. The network model and its associated input parameters appear in the directory structure specific to the ensemble, e.g., TEVA-SPOT-Database/Collection Name/BaseEnsemble. 
- The TEVA-SPOT archival feature provides the ability to archive ensemble data files while preserving the ensemble definition and description along with the results. All archived data are fully retrievable, if needed.

Data Analysis and Report Preparation TEVA-SPOT provides dose-over-threshold results in a text file with column number 1 corresponding to the node identification name and columns 4 through $\mathrm{N}$ corresponding to the impacts for the dose thresholds specified in TEVA-SPOT. The thresholds or dose levels appear as a header in row number 1.

TEVA-SPOT provides some statistical analyses. For example, the identification of the $10^{\text {th }}$, $25^{\text {th }}, 50^{\text {th }}, 75^{\text {th }}, 90^{\text {th }}$, and $100^{\text {th }}$ percentile scenarios (identification of injection node) for each ensemble and the associated receptors affected are provided by TEVA-SPOT. Additional analyses were developed using Microsoft Excel ${ }^{\circledR}$ and R (R Development Core Team 2010). All plots in this report, e.g., scatter and histogram, were developed separately from TEVA-SPOT using R. Selected results and statistical analyses were independently verified using hand calculations. Maps were created by exporting health impacts assessment results as dBase ${ }^{\circledR}$ IV data files from TEVASPOT and then merging and mapping the results in ArcGIS ${ }^{\circledR}$ 9.3. Report writing was version controlled with a revision number and date. Reviewer comments were addressed and tracked by revision number. The report was prepared using $\mathrm{ET}_{\mathrm{E}} \mathrm{X} 2_{\varepsilon}$ (LaTeX Project 2010). Report formatting is consistent with EPA guidelines (U. S. EPA 2010b). 